\title{
Optimization and application of Trim-Away for studying a liquid-like spindle domain in mammalian oocytes
}

\section{Dissertation}

\author{
for the award of the degree
}

"Doctor rerum naturalium"

of the Georg-August-Universität Göttingen

within the doctoral program Physics of Biological and Complex Systems

of the Georg-August-Universität Göttingen

submitted by

\section{Chun So}

from Hong Kong

Göttingen, 2019 


\section{Thesis Committee}

Dr. Melina Schuh

Department of Meiosis, Max Planck Institute for Biophysical Chemistry

Prof. Dr. Stefan Jakobs

Structure and Dynamics of Mitochondria, Max Planck Institute for Biophysical Chemistry

Prof. Dr. Jörg Enderlein

Biophysics / Complex Systems, III. Physical Institute, Georg-August-Universität Göttingen

\section{Members of the Examination Board}

Dr. Melina Schuh

Department of Meiosis, Max Planck Institute for Biophysical Chemistry

Prof. Dr. Stefan Jakobs

Structure and Dynamics of Mitochondria, Max Planck Institute for Biophysical Chemistry

\section{Further members of the Examination Board}

Prof. Dr. Jörg Enderlein

Biophysics / Complex Systems, III. Physical Institute, Georg-August-Universität Göttingen

Prof. Dr. Rüdiger Behr

Degenerative Diseases, German Primate Center

Dr. Roland Dosch

Department of Developmental Biochemistry, University Medical Center Göttingen

Prof. Dr. Markus Zweckstetter

Structure Determination of Proteins Using NMR, Max Planck Institute for Biophysical Chemistry

\section{Date of oral examination: $19^{\text {th }}$ August 2019}


"Omne vivum ex ovo"

- William Harvey

"The gift of the great microscopist is the ability to think with the eyes and see with the brain. Deep revelations into the nature of living things continue to travel on beams of light."

- Daniel Mazia 


\section{Acknowledgement}

First of all, I would like to thank my supervisor Dr. Melina Schuh for giving me the opportunity to work together and building my motivation towards the oocyte world. I will not be able to publish two impactful manuscripts within three years without her infinite patience and continuous guidance. I am grateful to the Croucher Foundation for the generous financial support in the past three years. I am also grateful to Prof. Dr. Stefan Jakobs and Prof. Dr. Jörg Enderlein for being a supportive thesis advisory committee member, Prof. Dr. Rüdiger Behr, Dr. Roland Dosch and Prof. Dr. Markus Zweckstetter for kindly participating in my examination board.

I would also like to thank members of the Schuh lab, especially Bianka Seres for teaching me microinjection, for her help with human oocytes and for always cheering me up, Eike Mönnich for his help with analyses using Imaris and MATLAB, Anastasija Pejkovska for her help with protein purification, Tommaso Cavazza for sharing cow oocytes and lots of discussion on microtubules, Shiya Cheng for lots of discussion on phase separation, Julia Uraji and Lydia Abdelhalim for their help with human oocytes, Martina Daniel, Eirini Bellou and Katarina Harasimov for their help with pig and sheep oocytes, Lena Wartosch and Daniela Wassermeyer for their help with paperwork and my life outside the lab. Special thanks to Peter Lenart for lots of discussion on fluorescence microscopy, Wiebke Möbius and Anna Steyer for their help with electron microscopy, Sven Truckenbrodt for his help with expansion microscopy, Anthony Hyman, Titus Franzmann and Xiaojie Zhang for lots of discussion on phase separation. Equally, I am grateful to the animal facility at MPIBPC, patients and embryology teams at Bourn Hall Clinic and Kinderwunschzentrum, slaughterhouses and all the labs sharing plasmids and antibodies with me. My work will not be possible without their selfless contribution of materials. 
Finally, I would like to thank my past colleagues, my friends and my family. Thank you all my past colleagues at the Chinese University of Hong Kong, Fudan University, National University of Singapore, University of Oxford and Academia Sinica for teaching me all the different wetlab techniques, otherwise I would not have been where I am today. Faye, thank you for all the encouragement when my life in Germany went astray and for always reminding me why I started. Jayden, TLC, Yiyue, Shane and Gary and Phil, thank you for always being a good listener. Tiffany, thank you for supporting me during the past six months of endless paper and thesis writing, and for showing up in my life just at the right moment. Mum and Dad, thank you for respecting my pick for Germany, and for bearing with my bad temper whenever life was not going well in Germany. I dedicate this thesis to all my loved ones who were there for me in the past three years. 


\section{Abstract}

In this thesis, I reported a membraneless structure that permeated a major region of the female meiotic spindle and formed droplet-like protrusions around the spindle poles. Proteins within this previously unknown structure were highly dynamic and could redistribute rapidly throughout the entire spindle region. I found that this unusual structure behaves similar to a liquid and forms by phase separation and hence, termed it the liquid-like meiotic spindle domain (LISD). Interestingly, the LISD was not only present in the spindle of mouse oocytes, but also cow, pig and sheep oocytes and is thus widely conserved among mammals. Similar structures were not observed in wildtype or centrosome-depleted somatic cells, suggesting that the LISD is likely exclusive to the specialized spindle in oocytes. To identify the key proteins driving LISD assembly, I further optimized Trim-Away, the technique for acute depletion of endogenous proteins in mammalian cells. By combining in vitro and in vivo assays, I identified Aurora A kinase and two LISD proteins, transforming acidic coiled-coil-containing protein 3 (TACC3) and clathrin heavy chain 17 (CHC17), as the proteins essential for LISD assembly. Disruption of the LISD via different means released microtubule regulatory factors that reside within this domain into the cytosol and caused severe spindle defects. Spindles were half of their original size and failed to segregate chromosomes properly. Microtubule growth rates were reduced, and their overall turnover was increased. Both kinetochore fibers as well as interpolar microtubules were strongly depleted. A TACC3 mutant that loses its ability to phase-separate failed to rescue the substantial loss of microtubules in TACC3-depleted oocytes. Together, these data suggest that the LISD is necessary for the efficient assembly of stable acentrosomal spindle in mammalian oocytes. 


\section{Table of Contents}

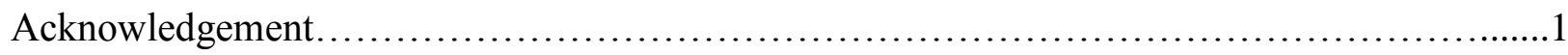

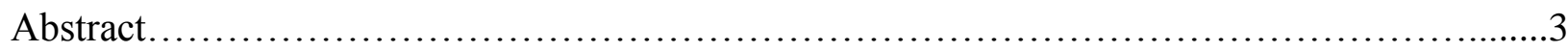

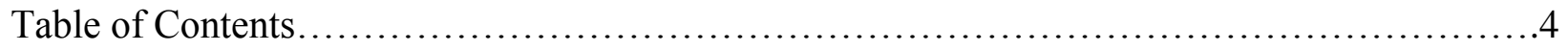

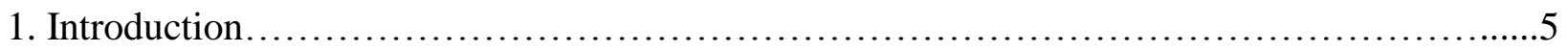

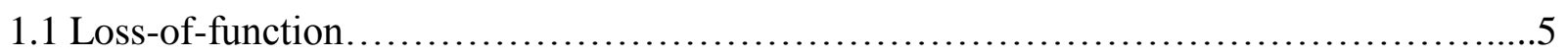

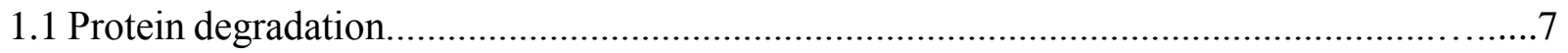

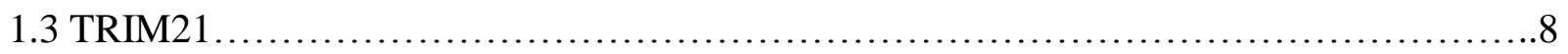

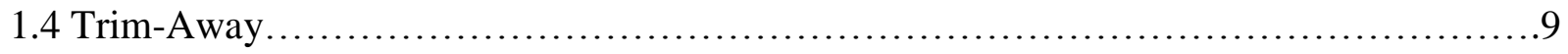

1.5 Mammalian oocyte development................................................ 11

1.6 Meiosis in mammalian oocytes.......................................................

1.7 Spindle assembly in mammalian oocytes........................................... 15

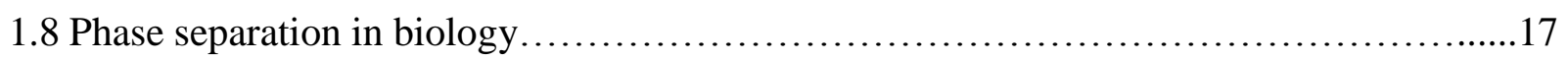

1.9 Phase separation in centrosome and microtubule assembly .............................19

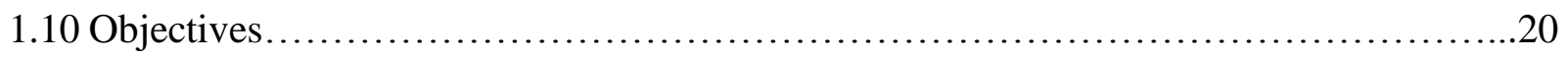

2. Publication 1 (Acute and rapid degradation of endogenous proteins by Trim-Away)...........22

3. Publication 2 (A liquid-like spindle domain promotes acentrosomal spindle assembly in

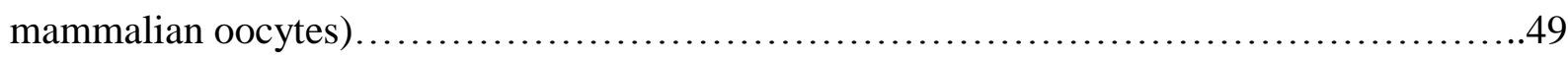

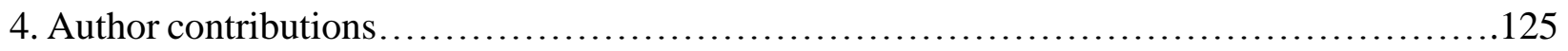

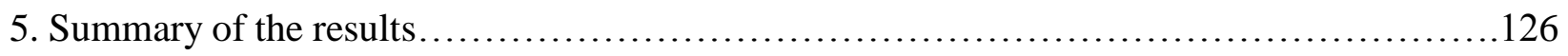

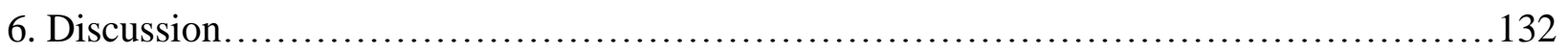

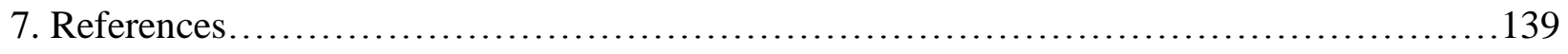

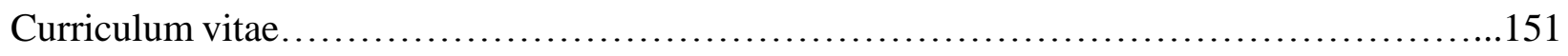




\section{Introduction}

\subsection{Loss-of-function}

Loss-of-function is a genetic approach that has been extensively used to study protein functions in cells, tissues and even whole organisms ${ }^{1}$. Loss-of-function methods reduce or ablate protein function by either interfering with protein synthesis or inducing protein degradation ${ }^{1}$. At the genomic level, protein-coding genes can be disrupted by mutagenesis or genome editing ${ }^{1}$. Lossof-function mutation can be introduced using either chemical mutagens or transposons ${ }^{1}$. Genome editing technologies such as zinc-finger nucleases (ZFNs), transcription activator-like effector nucleases (TALENs) and the clustered regularly interspaced short palindromic repeats (CRISPR)Cas system are all based on endonucleases that can be engineered to bind to a specific DNA sequence and introduce a frameshift or nonsense mutation, or delete part of the coding sequence ${ }^{2}$. At the transcription level, messenger RNA (mRNA) synthesis can be perturbed using transcription activator-like effector (TALE) repression or CRISPR interference (CRISPRi) ${ }^{3}$. In both cases, TALE and Cas are fused with a transcriptional repressor instead of a nuclease ${ }^{3}$. At the posttranscriptional level, RNA interference (RNAi) can target mRNAs for degradation using sequencespecific short hairpin RNAs (shRNAs) or small interfering RNAs (siRNAs) ${ }^{3}$. Once Dicer processed the shRNAs into siRNAs, which are then loaded onto Argonaute proteins, the resulting RNA-induced silencing complexes bind to and degrade the target mRNAs ${ }^{4}$. At the translational level, morpholinos can be used to block mRNA translation ${ }^{5}$. These antisense oligomers bind to the target mRNAs and sterically block the progression of the translation initiation complex from the 5' cap to the start codon ${ }^{5}$. 
Although the aforementioned techniques have been proven to be useful for studying many different proteins in various model organisms, there are several limitations. One common major limitation is that they do not act on already-synthesized proteins inside the cells. Whereas most proteins are being constantly degraded and subsequently replaced with newly synthesized proteins, recent studies found that some proteins can persist for years in both metabolically inactive and long-lived metabolically active cells ${ }^{6,7}$. Once these proteins are trafficked to certain organelles such as the mitochondria and endoplasmic reticulum or incorporated into essential cellular structures such as the cohesin and nuclear pore complexes, they turnover slower and thus become resistant to lossof-function techniques that target protein synthesis ${ }^{8}$. Another limitation is that actual protein depletion typically takes days after the application of these techniques. This makes them unsuitable for studying short-lived biological processes such as membrane trafficking, cell division and early embryonic development. Also, interpretation of the results could become complicated as the phenotypes can be either a direct consequence or a secondary consequence of earlier defects after protein depletion ${ }^{9}$. Furthermore, the delay in between may allow cells to activate compensatory mechanisms, modifying or even masking the expected phenotypes ${ }^{10}$.

To overcome these limitations, at least partially, several techniques have been developed to directly deplete the target proteins. A few ligand- and peptide-based techniques have been developed to allow protein degradation without the need of tagging the proteins, however, not all proteins can be targeted with these methods ${ }^{11,12}$. Many protein degradation techniques are based on recruiting SKP1-CUL1-F-box (SCF) E3 ubiquitin ligases to the target proteins, such as the binding of the F-box protein TIR1 to proteins tagged with an auxin-inducible degron and the binding of the GFP nanobody/F-box domain of Slmb fusion to GFP-tagged proteins ${ }^{13,14}$. Likewise, 
ELOC-CUL2-SOCS-box (ECS) E3 ubiquitin ligases have been exploited for similar purpose ${ }^{15}$. Other protein degradation techniques are based on modulating protein stability, for instance, by fusing the target protein to a destabilizing mutant of FKBP12 or to a HaloTag with hydrophobic moiety ${ }^{16,17}$. In addition to degradation, proteins can be inactivated by knock-sideways, in which proteins are rerouted from their principal site of action to the mitochondria upon chemical-induced dimerization between the target proteins and the tethering proteins ${ }^{18}$. Most of these techniques act acutely, typically in the range of minutes. Nevertheless, they are not applicable to endogenous proteins and hence, unsuitable for applications in nondividing primary cells, which would otherwise require the generation of transgenic animals.

\subsection{Protein degradation}

Ubiquitin-proteasome system (UPS) and autophagy are two major protein degradation pathways in mammalian cells. Whereas the UPS is responsible for the degradation of small, short-lived proteins, autophagy is the preferred route for large, heterogeneous cytoplasmic materials which cannot fit into the proteasomes ${ }^{19}$.

Ubiquitin is a small, highly conserved protein, which can be covalently attached to the $\varepsilon$-amino group of a lysine residue ${ }^{19}$. In the UPS pathway, E1 ubiquitin-activating enzyme first activates the Cterminal glycine of ubiquitin and transfers it to the E2 ubiquitin-conjugating enzyme ${ }^{19}$. Substrate-specific E3 ubiquitin ligase then brings charged E2 and the substrate in close proximity, and facilitate the transfer of ubiquitin ${ }^{19}$. Substrates that are polyubiquitinated with K48-linked chains are recognized by ubiquitin receptors, which target the substrates to the proteasomes for degradation ${ }^{19}$. 
Depending on how the cytosolic cargoes are directed to the lysosomes, the autophagy pathway can be further classified into chaperone-mediated autophagy (CMA), microautophagy and macroautophagy ${ }^{19}$. In CMA, HSC70 recognizes cytosolic proteins that expose a pentapeptide signature motif (KFERQ) and in turn binds to LAMP2A on the lysosomes ${ }^{19}$. The substrates are then unfolded and translocated into the lysosomes for degradation, which is assisted by a luminal form of $\mathrm{HSC}{ }^{19}$. In microautophagy, cytosolic content is invaginated by the late endosomal membrane, which then pinches off into the lumen in an ESCRT-dependent manner ${ }^{20}$. In macroautophagy, cytosolic material is engulfed by the double-membrane phagophore, which elongates and closes to form the autophagosome ${ }^{19}$. Mature autophagosome is transported along microtubules towards microtubule-organizing center, where its outer membrane fuses with lysosomes or late endosomes to form autolysosome ${ }^{19}$. Ultimately, the cargoes are degraded by lysosomal hydrolase ${ }^{19}$. Depending on whether cargo recognition is involved or not, microautohpahy and macroautophagy can be selective or non-selective (bulk) ${ }^{19,20}$.

\subsection{TRIM21}

TRIM21 is an Fc receptor that is widely expressed by cells of most histogenic lineages and belongs to the tripartite motif containing (TRIM) protein family ${ }^{21,23-25}$. All TRIM members share an Nterminal RING E3 ubiquitin ligase domain, one or two B Box domains and a coiled-coil domain ${ }^{21-}$ 26. Approximately half of all known TRIM proteins possess an additional C-terminal PRYSPRY domain, which determines ligand specificity and function ${ }^{21-26}$. The PRYSPRY domain of TRIM21 binds to residues on the $\mathrm{Fc}$ of immunoglobulin (Ig) at the $\mathrm{C}_{\mathrm{H} 2}-\mathrm{C}_{\mathrm{H} 3}$ domain interface, an epitope overlapping with FcRn, protein A and protein $\mathrm{G}$ but not $\mathrm{Fc} \gamma \mathrm{R}$ and $\mathrm{C1} \mathrm{q}^{21,23-26}$. The coiled-coil domain facilitate TRIM21 dimerization in an anti-parallel manner and allows simultaneous 
binding of both heavy chains, increasing its affinity for Ig through avidity ${ }^{21-26}$. TRIM21 binds Fc independent of $\mathrm{pH}$, and it displays cross-reactivity with $\mathrm{IgG}$ from a range of mammalian species $^{21,23}$. Although TRIM21 is the IgG receptor with the highest affinity in human, it also binds to $\operatorname{IgM}$ and $\operatorname{Ig}$ A with lower affinity ${ }^{21,23,25,26}$.

In the presence of intracellular antibody-bound pathogens, TRIM21 first recruits E2 UBE2W, which catalyze the automonoubiquitination of TRIM21 ${ }^{23-26}$. E2 heterodimer UBE2N/2V2 is then recruited and uses the monoubiquitination to prime anchored K48 and K63 polyubiquitin chain extension $^{23-26}$. The unfoldase/segregase AAA ATPase p97/VCP subsequently interacts with K48linked chains, and proteasome-associated deubiquitinase Poh1 liberates K63-linked chains from TRIM21 $1^{23-26}$. These allow the translocation of the TRIM21/immune complex into the proteolytic

core of the proteasome ${ }^{23-26}$. The release of free K63-linked chains activates TAK1-TAB1-TAB2 and IKK $\alpha$-IKK $\beta$-NEMO kinase complexes, coupling antibody-dependent intracellular neutralization with the activation of transcription factors NF- $\kappa \mathrm{B}, \mathrm{AP}-1$ and IRF $3 / 5 / 7^{23-26}$.

\subsection{Trim-Away}

In 2017, our lab developed a depletion technique for endogenous proteins, which we called 'TrimAway $^{27}$. Trim-Away exploits TRIM21 to degrade endogenous proteins targeted by specific antibodies delivered into the cells ${ }^{27}$. A typical Trim-Away experiment involves two steps. (1) Expression of TRIM21. Although TRIM21 is broadly expressed in many different cell types, the endogenous level may not be sufficient for complete target protein degradation as TRIM21 is codegraded during the experiment. Additional TRIM21 can be introduced in the form of transient 
transfection, stable transfection, mRNA or recombinant protein ${ }^{27}$. (2) Delivery of antibody. Antibody can be delivered into the cells by microinjection or electroporation using the Neon Transfection System ${ }^{27}$. Alternatively, nanobody-Fc fusion can be used, which can even be delivered in the form of transfection or $\mathrm{mRNA}^{27}$.

Trim-Away has several unique advantages over the previous protein depletion techniques. First, Trim-Away directly degrade endogenous proteins. It does not rely on inherent turnover of the target protein and is effective against long-lived proteins. Second, Trim-Away does not require prior modification of the target protein. It is therefore applicable to nondividing primary cells and also primary immune cells that have active nucleic-acid sensing mechanisms. Third, unlike E3s such as VHL used in PROTAC, TRIM21 is not known to have cellular housekeeping function. It is less likely that there is competition with endogenous substrates, and normal metabolism is affected. Although the TRIM21 pathway could activate immune signaling, thanks to its high activation barrier, transcription factors such as NF- $\mathrm{BB}$ is only weakly activated in Trim-Away experiments $^{27}$.

Successful applications of Trim-Away include degradation of long-lived cohesin REC8 in mouse oocytes, degradation of intracellular signaling molecule NLRP3 in human primary macrophages, selective degradation of signaling pathway components and selective degradation of protein mutants ${ }^{27}$. More recently, apart from mammals, Trim-Away has also been successfully applied in zebrafish $^{28}$. 


\subsection{Mammalian oocyte development}

At embryonic day 6 post conception (E6.5) in mouse, or the fourth-fifth week in human, the extraembryonic ectoderm and visceral endoderm subject a subset of epiblastic cells from the primitive embryonic ectoderm to induction by intense BMP4 signaling ${ }^{29,30}$. This specifies their entry into germ lineage ${ }^{29,30}$. At E8.5 in mouse, or between the fifth and eighth week in human, these nascent primordial germ cells (PGCs) expresses pluripotency markers, undergoes epigenetic changes resembling that of pluripotent stem cells, and begin their migration into the posterior end of the primitive streak, which later becomes the extraembryonic mesoderm ${ }^{29,30}$. Nascent PGCs initially migrate through the allantois and reside temporarily in the yolk sac ${ }^{29,30}$. They then migrate caudally through the hindgut towards the dorsal mesentery and finally colonize the urogenital ridges $^{29,30}$. Throughout the migration, PGCs proliferate rapidly ${ }^{30}$.

Upon their arrival, nascent PGCs switch off the pluripotency program and switch on sex-specific genes $^{30}$. The urogenital ridges, thickenings of coelomic epithelium superimposed on the anterior portion of the mesonephros, develop into the gonadal primordia in a sex-dependent manner and become the chief site for PGC development ${ }^{29,30}$. In XY gonads, transcription factor SRY is expressed and binds to TESCO, which upregulates SOX9 and induces differentiation of Sertoli cells, driving testicular formation ${ }^{29,30}$. In XX gonads, where SRY is not expressed, WNT4 and RSPO1 maintains $\beta$-catenin signaling and drives ovarian formation ${ }^{29,30}$. Once PGCs complete genome reorganization, they continue mitosis to produce a large amount of oogonia in the female ${ }^{29}$. 
At E10.5 in mouse, or between week 10 and 20 in human, oogonia undergo multiple mitotic divisions that do not complete cytokinesis, forming transitory cysts known as germ cell nests ${ }^{29-31}$. These nests are clusters of oogonia interconnected by cytoplasmic bridges and enveloped by somatic cells ${ }^{29-31}$. At E13.5 in mouse, or between week 11 and 13 in human, secretion of retinoic acid from adjacent mesonephros triggers the induction of meiosis in an anterior to posterior wave $^{29,30}$. Oogonia enter prophase I through leptotene, zygotene and pachytene, that characterize pairing of homologous chromosomes, synapsis and recombination, and arrest at diplotene stage as primordial oocytes $^{29-31,33}$. Shortly after birth in mouse, or between week 16 and 21 in human, some cells die through apoptosis, destabilizing and rupturing these nests ${ }^{29-31}$. Outlying somatic cells then invade these nests and surround oocytes of less than $20 \mu \mathrm{m}$ in diameter, forming primordial follicles ${ }^{29-33}$. Indeed, two-thirds of primordial oocytes undergo apoptosis in a phenomenon known as the apoptotic wave ${ }^{29}$. Only the cells that survive the apoptotic wave constitute the final pool of primordial follicles that are available for the entire reproductive lifespan in the female ${ }^{29}$.

Primordial follicles constitute the ovarian quiescent follicle reserve $\mathrm{e}^{29,30}$. Throughout the reproductive lifespan, primordial follicles are continuously released from dormancy and recruited to the growing pool $^{29,30}$. The pre-antral phase of folliculogenesis is largely independent on extraovarian hormonal stimuli, but the local production of growth hormones ${ }^{30,32}$. From primordial follicles to primary follicles, oocytes are surrounded by zona pellucida and enlarge to more than $20 \mu \mathrm{m}$ in diameter, and squamous (pre-)granulosa cells transform into cuboidal granulosa cells ${ }^{30-}$ 32. From primary follicles to secondary follicles, meiotically incompetent oocytes further increase in size, granulosa cells proliferate to from multiple layers, and thecal cells are recruited to the basal lamina surrounding the granulosa cells ${ }^{30,33}$. Once pre-antral follicles reach a species-specific size, 
granulosa cells secrete glycoproteins and create small fluid-filled cavities, which ultimately coalesce into a single cavity known as the antrum ${ }^{30,33}$.

To progress through the antral and pre-ovulatory phase, follicles become dependent on the cyclical pituitary secretion of $\mathrm{FSH}$ and $\mathrm{LH}^{30,32,33}$. At the antral stage, meiotically competent oocytes cease to grow and suppress the expression of LH receptors in cells surrounding them, regulating the phenotypic differentiation of granulosa cells into cumulus granulosa cells and mural granulosa cells $^{30,32,33}$. Other than providing metabolic support, cumulus granulosa cells that are in close contact with the oocyte through gap junctions also maintain the oocyte in meiotic arrest ${ }^{30,32,33}$. By contrast, mural granulosa cells line the inner side of the basal lamina and are responsible for steroidogenesis $^{30,33}$. Whereas most antral follicles will undergo atretic degeneration, only a subset of follicles known as the dominant follicles reach the pre-ovulatory phase ${ }^{30}$. Mural granulosa cells of these follicles, but not the oocytes, express high level of LH receptors, making them responsive to the pre-ovulatory LH surge ${ }^{30}$.

\subsection{Meiosis in mammalian oocytes}

Fully grown oocytes remain arrested in the diplotene stage as dictyate due to the low activity of the maturation-promoting factor (MPF), which is a complex of the kinase subunit CDK1 and the regulatory subunit cyclin B1 that regulates $\mathrm{G} 2 / \mathrm{M}$-transition ${ }^{30,34-36}$. The low activity of MPF is maintained by three mechanisms: (i) High level of intra-oocyte cAMP promotes the activation of PKA that stimulates nuclear WEE1B/WEE2 and cytoplasmic MYT1 kinases, which in turn inactivate $\mathrm{CDC} 25 \mathrm{~B}$ phosphatase required for $\mathrm{CDK} 1$ dephosphorylation and its resultant 
activation $^{29,30,34-36}$; (ii) CDC14B phosphatase counteracts CDK1 activity and activates nuclear APC/C coactivator CDH1, which constantly promotes cyclin B1 degradation by APC/C $30,34-36$; (iii) Cyclin B1 and CDC25B are excluded from the nucleus ${ }^{35,36}$. The high level of intra-oocyte cAMP is attributed by: (i) activation of the $\mathrm{G}_{\mathrm{s}}$ by $\mathrm{G}$-protein-coupled receptor on the oolemma and the subsequent stimulation of adenylyl cyclase in the oocyte ${ }^{30,34-36}$; (ii) transfer of cAMP produced in cumulus granulosa cells to the oocyte via gap junctions ${ }^{30,35}$; (iii) induction of guanylyl cyclase NPR2 in cumulus granulosa cells by the oocyte ${ }^{34}$. Secretion of NPPC by mural granulosa cells activates NPR2 that synthesizes cGMP, which enters the oocyte via gap junctions and inhibit oocyte-specific PDE3A that degrades cAMP ${ }^{30,34-36}$.

Following puberty, cyclic pre-ovulatory LH surge resumes meiosis. Binding of LH to its receptor on mural granulosa cells reduces NPPC secretion, and leads to the activation of $\mathrm{G}_{\mathrm{s}}$ and subsequently adenylyl cyclase $\mathrm{e}^{34,35}$. The production of cAMP promotes the activation of PKA, leading to the synthesis and release of EGFR ligands ${ }^{34}$. EGFR signaling decreases the level of cGMP and activates MAPK, which promotes the phosphorylation of connexin 43 and reduces gap junction permeability, preventing the influx of cGMP ${ }^{29,30,34-36}$. As a result of increased cAMP hydrolysis by PDE3A, reduced level of intra-oocyte cAMP relieves PKA-mediated inactivation of CDC25B, resulting in the activation of $\mathrm{CDK} 1^{29,30,34-36}$. The activation of CDK1 is further reinforced by (i) concomitant nuclear translocation of cyclin B1 and CDC25B that causes activation of a fraction of MPF, which in turn promotes the export of WEE1B/WEE2 to the cytoplasm $^{35,36}$; (ii) EMI1-mediated inhibition of APC ${ }^{\mathrm{CDH} 1}$, which counteracts $\mathrm{CDC} 14 \mathrm{~B}^{34,35}$. 
Unlike the rapid, switch-like activation of CDK1 in mitosis, the slow rise in CDK1 activity, which results in a prometaphase I lasting for 6 to 7 hours in mouse oocytes, is accompanied by the progressive accumulation of cyclin $\mathrm{B} 1^{36}$. This is achieved partially by the de novo translation of cyclin B1 mRNAs, and partially by the suppression of APC/C activity by CDK1 and MAPK ${ }^{36}$. CDK1 activity peaks at metaphase, when bivalents are properly aligned on the metaphase plate ${ }^{36}$. Once bivalents are correctly attached to kinetochore microtubules from the two spindle poles and the spindle assembly checkpoint is satisfied, anaphase onset is initiated ${ }^{36}$. The loss of SACmediated repression of $\mathrm{APC} / \mathrm{C}$ and the association of $\mathrm{APC} / \mathrm{C}$ with its coactivator $\mathrm{CDC} 20$ promote the degradation of securin and cyclin B1, which in turn promotes the cleavage of cohesin by separase and the lowering of CDK1 activity, respectively ${ }^{36}$.

Following chromosome segregation and polar body extrusion, CDK1 activity is rapidly upregulated to promote entry into prometaphase II $^{29,34,36}$. Oocytes arrest at metaphase II as a result of $\mathrm{APC}^{\mathrm{CDC} 20}$ inhibition by cytostatic factor (CSF), which is an activity of the MOS/MEK1/MAPK/p90 ${ }^{\text {Rsk }}$ and EMI2 pathways ${ }^{29,34,36}$. Other pathways that act directly upon CDK1 also operates ${ }^{36}$. CSF-mediated stabilization of MPF prevents parthenogenetic activation and hence, development of embryos without the contribution of paternal genome ${ }^{29}$.

\subsection{Spindle assembly in mammalian oocytes}

The female meiotic spindle from mammalian oocytes was first observed at ultrastructural level in the mouse back in the $1970 \mathrm{~s}^{37}$. Although the pathways responsible for microtubule nucleation in mammalian oocytes have been identified in the past 50 years, little is known about how 
microtubules are assembled into the female meiotic spindles, which are of a size larger than a somatic cell.

Unlike somatic cells, in which centrosomes dominate the assembly of mitotic spindles, mammalian oocytes do not possess centrosomes and assemble acentrosomal spindles ${ }^{38}$. Centrosomes are composed of a pair of centrioles and the pericentriolar material (PCM), which contains factors for microtubule nucleation and anchoring ${ }^{39}$. In mouse oocytes, centrioles are lost after the pachytene stage and the PCM persists as acentriolar microtubule organizing centers (aMTOCs) ${ }^{37,40}$. In oocytes from other mammals, centrioles are lost and the PCM is dispersed, degenerating the centrosomes completely ${ }^{38}$. Why mammalian oocytes eliminate their centrioles? One hypothesis is that centriole elimination in oocytes balances the number of centrosomes after the introduction of sperm centrioles, preventing the formation of multipolar spindle in the first embryonic division ${ }^{38}$. Another hypothesis is that centriole elimination prevents parthenogenetic activation, as microinjected centrosomes functions as zygotic centrosomes and induces activation in Xenopus $\operatorname{eggs}^{38}$

In mouse oocytes, aMTOCs form de novo from an interphase-like microtubule network in prophase and exist as large clusters around the nucleus at the germinal vesicle (GV) stage ${ }^{40-42}$. Prior resumption of meiosis, CDK1 phosphorylates a wide range of substrates that mediate germinal vesicle breakdown (GVBD), chromosome condensation and cytoskeleton remodeling ${ }^{35,36}$. PLK1 decondenses the aMTOCs, which are stretched along the nuclear envelope in a BICD2/dynein-dependent manner upon $\mathrm{GVBD}^{40,42}$. After GVBD, aMTOCs are further fragmented by KIF11 $1^{42}$. Although aMTOCs nucleate few microtubules upon GVBD, they are 
responsible for initial microtubule nucleation in mouse oocytes and their nucleation capacity progressively increases during meiosis $\mathrm{I}^{40,41}$. Meanwhile, as chromosome condenses, the Ran guanosine exchange factor RCC1 on chromosomes converts inactive Ran-GDP into active Ran$\mathrm{GTP}^{43}$. The Ran-GTP gradient in turn promotes the dissociation of spindle assembly factors from their inhibitory binding to importins, contributing to later microtubule nucleation ${ }^{43}$. Disruption of aMTOCs by depleting PCM components or inhibition of Ran-GTP with a dominant-negative mutant alone did prevent spindle assembly ${ }^{41,44,45}$. Nevertheless, no spindle was formed upon RanGTP inhibition in aMTOCs-disrupted oocytes, suggesting that both pathways are essential for spindle assembly ${ }^{45}$. Upon initiation of nucleation, the initial stochastic distribution of aMTOCs results in a ball of microtubules, with newly individualized chromosomes having their kinetochores orienting inwards ${ }^{41}$. As the microtubule ball elongates into a barrel-shaped array in a KIF11-dependent manner, chromosomes form a loose ring that marks the equator of the forming ellipse, termed the prometaphase belt, and HURP mediates the sorting of aMTOCs to the two newly formed spindle poles ${ }^{41,46,47}$. Although aMTOCs cluster and facilitate bipolar spindle assembly, they are not absolutely essential for spindle pole formation ${ }^{45,48}$. Finally, chromosomes invade the spindle and form the metaphase plate ${ }^{41}$.

For human oocytes, in which aMTOCs were not detected, the Ran-GTP pathway is absolutely essential for initiation of microtubule nucleation ${ }^{49}$. However, whether this applies to other nonmurine oocytes and how their spindles are assembled in the absence of aMTOCs remain unknown.

\subsection{Phase separation in biology}


Classic organelles, such as the nucleus, endoplasmic reticulum, Golgi apparatus and mitochondria, are compartments best known for their surrounding membrane. Indeed, many cellular compartments, such as the nuclear bodies and RNA granules lack a physical barrier separating their internal components from the surrounding environment. How these membrane-less compartments sequester their components and maintain their structure in the cytosol remained elusive for years until the $\mathrm{P}$ granules were discovered to be liquid-like.

$\mathrm{P}$ granules are perinuclear RNA granules in the germ cells of Caenorhabditis elegans embryos ${ }^{50}$. In 2009, careful inspection revealed that they have the following properties reminiscent of liquids: (i) they are spherical; (ii) they can fuse after touching and revert back to a spherical shape; (iii) they deform in shear flows; (iv) they rapidly exchange components with the cytosol and undergo internal rearrangement ${ }^{50}$. Since then, other compartments such as the nucleoli and stress granules were also found to display liquid-like properties ${ }^{51,52}$. Thus, phase separation via liquid-liquid demixing is proposed to form these compartments.

Phase separation refers to the change that occur as molecules transit from one configuration (phase) to another and the two phases are separated from one another ${ }^{53,54}$. Phase separation is typically referred to liquid demixing in biology ${ }^{53,54}$. When the interactions between molecules are sufficiently stronger than the interactions between the molecule and the solvent, its energetics overcome the entropic tendency of the solution to remain homogenously mixed ${ }^{53,54}$. Consequently, the mixture separates into a dilute phase of larger volume and lower concentration and a condensed phase of smaller volume and higher concentration ${ }^{53,54}$. As the chemical potential in both phases is equal, this eliminates non-diffusive flux while allows diffusion across the phase boundary ${ }^{53,54}$. At 
equilibrium, the concentration difference between the two phases is maintained without constant input of energy ${ }^{53,54}$. In biological systems, phase separation of proteins is driven by multivalency, which can be achieved with (i) multiple modular interaction domains and (ii) large intrinsically disordered regions that lack a defined tertiary structure but contain low complexity sequence that provide short-lived interactions with low affinity and no stereospecificity ${ }^{54}$ This is in sharp contrast to the relatively long-lived interactions with high affinity and stereospecificity that drives oligomerization and polymerization, which can arrest the dynamics within ${ }^{54}$.

There are several functional implications for phase separation in biology. First, phase separation can substantially increase the local concentration of reactants, thereby accelerating rate limiting steps in reactions ${ }^{53,54}$. Second, phase separation can selectively concentrate some reactants and exclude the others, conferring specificity to reactions ${ }^{54}$. Third, phase separation can sequester components from the cytosol, inhibiting their activity or buffering their concentration in the $\operatorname{cytosol}^{53,54}$. Four, all the aforementioned functions can be switched on and off quickly by condensation and dissolution of the phase ${ }^{54}$.

\subsection{Phase separation in centrosome and microtubule assembly}

Although tubulin itself does not undergo phase separation but polymerizes through stereospecific interactions, phase separation of centrosomal and spindle-related proteins have recently been implicated in centrosome and microtubule assembly, potentially contributing to mitotic spindle assembly $^{55}$. 
For centrosome assembly, C. elegans PCM scaffold protein SPD-5 was first found to phaseseparate in vitro ${ }^{56}$. SPD-5 condensates morphologically and dynamically resemble mitotic PCM in vivo and can recruit 'clients' microtubule polymerase ZYG-9 and microtubule-stabilizing protein TPXL-1, concentrating tubulin $\sim 4$-fold to nucleate microtubule asters in vitro ${ }^{56}$. Likewise, phase separation of Xenopus PLK4 kinase was found to recruit tubulin and was proposed to recruit its substrate STIL and microtubule nucleator $\gamma$-tubulin, promoting de novo MTOC formation in Xenopus extracts ${ }^{57}$.

For microtubule assembly, several microtubule-binding proteins have been found to phaseseparate in vitro. One is the Xenopus lamin-B spindle matrix protein BuGZ, whose condensates were initially found to bundle microtubules and concentrate tubulin, promoting microtubule polymerization near existing microtubules ${ }^{58}$. Later, phase separation of BuGZ was also found to promote the activation of AURA, one of the kinases driving mitotic spindle assembly ${ }^{59}$. The neuronal protein Tau is another one whose condensates can co-condense tubulin and polymerize Tau-encapsulated microtubule bundles in vitro ${ }^{60}$. More recently, phase separation of microtubulestabilizing protein TPX2 was similarly found to co-condense tubulin, promoting microtubule polymerization from existing microtubules ${ }^{61}$. Although BuGZ and TPX2 have been implicated in mitotic spindle assembly and it is tempting to imply a role of their phase separation, there is no evidence that they exhibit liquid-like properties in vivo ${ }^{55}$.

\subsection{Objectives}


Three years ago, when I started my doctoral study with the project 'Studies of individual chromosome behavior in mammalian oocytes', I wanted to establish a fluorescent reporter for kinetochore fibers (k-fibers) to image kinetochore-microtubule interactions in live oocytes. One candidate is TACC3, which forms intermicrotubule bridges with $\mathrm{CHC} 17$ and chTOG to stabilize k-fibers in mitotic cells ${ }^{62}$. Surprisingly, expression of TACC3-mClover3 labeled not only k-fibers, but also a previously unknown structure on the female meiotic spindle in mouse oocytes. Interestingly, this structure permeated a major region of the spindle and formed droplet-like protrusions around the spindle poles. Therefore, I decided to rather focus on this structure, which was later termed the liquid-like meiotic spindle domain (LISD).

As the first goal of my doctoral study, I set out to perform a protein localization screen to identify other proteins residing within the LISD. In order to better understand the properties of the LISD, the second goal of my doctoral study was to characterize the LISD using different in vivo and in vitro assays. The third goal of my doctoral study was to identify the key components that drive LISD assembly. To partially achieve this goal, I optimized the Trim-Away technique to improve the survival rate, developmental rate and protein depletion efficiency in mammalian oocytes, which was the four goal of my doctoral study and led to my first publication (Acute and rapid degradation of endogenous proteins by Trim-Away) in Nature Protocols. Having the optimized tool, I progressed to the final goal of my doctoral study and investigated the role of the LISD in acentrosomal spindle assembly in mammalian oocytes. Ultimately, the successful completion of all five goals led to my second publication (A liquid-like spindle domain promotes acentrosomal spindle assembly in mammalian oocytes) in Science. 


\title{
Acute and rapid degradation of endogenous proteins by Trim-Away
}

\author{
Dean Clift ${ }^{1,4 \star}$, Chun So ${ }^{2,4}$, William A. McEwan ${ }^{1,3}$, Leo C. James ${ }^{1 \star}$ and Melina Schuh ${ }^{1,2,4 \star}$
}

Protein depletion is a key approach to understanding the functions of a protein in a biological system. We recently developed the Trim-Away approach in order to rapidly degrade endogenous proteins without prior modification. TrimAway is based on the ubiquitin ligase and Fc receptor TRIM21, which recognizes antibody-bound proteins and targets them for degradation by the proteasome. In a typical Trim-Away experiment, protein degradation is achieved in three steps: first, introduction of an antibody against the target protein; second, recruitment of endogenous or exogenous/ overexpressed TRIM21 to the antibody-bound target protein; and third, proteasome-mediated degradation of the target protein, antibody and TRIM21 complex. Protein degradation by Trim-Away is acute and rapid, with half-lives of 10-20 min. The major advantages of Trim-Away over other protein degradation methods are that it can be applied to any endogenous protein without prior modification; that it uses conventional antibodies that are widely available; and that it can be applied to a wide range of cell types, including nondividing primary human cells, for which other loss-of-function assays are challenging. In this protocol, we describe the detailed procedures for antibody preparation and delivery in mouse oocytes and cultured cells via microinjection and electroporation. In addition, we provide recommendations for antibody selection and validation, and for the generation of TRIM21-overexpressing cell lines for cases in which endogenous TRIM21 is limited. A typical Trim-Away experiment takes just a few hours.

Protein depletion is one of the key tools used in studying protein functions in cells and tissues, and can be achieved by either interfering with protein synthesis or inducing protein degradation. Protein synthesis can be blocked at various levels. At the genomic level, protein-coding genes can be disrupted using genome-editing technologies such as zinc-finger nucleases (ZFNs), transcription activator-like effector nucleases and the clustered regularly interspaced short palindromic repeats (CRISPR)-Cas system. All these technologies are based on nucleases that can be targeted to a specific DNA sequence and introduce a frameshift mutation or a premature stop codon, or delete the coding sequence ${ }^{1}$. At the post-transcriptional level, messenger RNAs (mRNAs) can be targeted for degradation using RNA interference (RNAi). To this end, sequence-specific small interfering RNAs or short hairpin RNAs are introduced into cells. These are incorporated into RNA-induced silencing complexes, which bind to and degrade the target $\mathrm{mRNA}^{2}$. At the translational level, morpholino oligomers can be used to block the translation of mRNAs. These antisense oligonucleotides bind to the target mRNA and block the progression of the translation initiation complex from the $5^{\prime}$ cap to the start codon ${ }^{3}$.

Although these techniques have been proven to be highly useful for studying various genes in different model systems, a common major limitation is that they are not suitable to deplete alreadysynthesized proteins. In both metabolically inactive and long-lived metabolically active cells, certain proteins-particularly those in essential cellular structures-can persist for years after translation ${ }^{4}$. These long-lived proteins cannot be depleted by blocking protein synthesis at the gene or mRNA level. Another limitation of methods that act at the gene or mRNA level is the long delay between the time of their application and actual protein depletion, which is typically in the range of days. These methods are therefore not well suited to investigating short-lived biological processes. For example, many regulatory proteins have multiple functions during different stages of mitosis, which takes only $\sim 1 \mathrm{~h}$ in $\mathrm{HeLa}$ cells ${ }^{5}$. If protein degradation is delayed, it is difficult to determine whether the phenotypes are a direct consequence of protein depletion or a secondary consequence of earlier

${ }^{1}$ Laboratory of Molecular Biology, Medical Research Council, Cambridge, UK. ${ }^{2}$ Department of Meiosis, Max Planck Institute for Biophysical Chemistry, Göttingen, Germany. ${ }^{3}$ Present address: UK Dementia Research Institute, Department of Clinical Neurosciences, University of Cambridge, Cambridge, UK. ${ }^{4}$ These authors contributed equally: Dean Clift, Chun So. *e-mails: dclift@mrc-Imb.cam.ac.uk; Icj@mrc-Imb.cam.ac.uk; melina.schuh@mpibpc.mpg.de 
defects $^{6}$. Delays may also allow cells to activate compensatory mechanisms, which may modify or even mask the phenotypes ${ }^{7}$.

To overcome these challenges, several methods have been developed that act directly at the protein level, targeting the protein itself for degradation. Some of these methods are based on controlling protein stability, for instance, by fusing the target protein to destabilizing domains that are controlled by ligands ${ }^{8,9}$. Others are based on recruiting SKP1-CUL1-F-box (SCF) E3 ubiquitin ligases to the target protein, such as the binding of an auxin-inducible degron to the F-box protein TIR1 and the binding of GFP-tagged proteins to a GFP nanobody fused to the F-box domain of Slmb ${ }^{10,11}$. Proteins can also be perturbed acutely by knock-sideways approaches, in which proteins are targeted away from their principal site of action, for instance, by tethering them to mitochondria ${ }^{12}$. However, all of these assays require the endogenous protein to be first replaced by a modified variant. Hence, they are not suitable for studying protein functions in all cell types. For instance, application of these methods in nondividing primary cells would often require the generation of transgenic animals. This is time consuming and not feasible for many species. For tag-free degradation, ligand- and peptide-based techniques have been developed, but the number of proteins that can be targeted with these methods is very limited ${ }^{13,14}$.

To achieve acute depletion of any endogenous protein without prior modification, we recently developed a post-translational protein depletion method, which we called 'Trim-Away'15. Trim-Away relies on an E3 ubiquitin ligase called TRIM21 (ref. ${ }^{16}$ ). TRIM21 is involved in the intracellular immune response; it binds to antibody-bound pathogens and proteopathic agents and targets them for degradation ${ }^{17-19}$. The precise mode of action of TRIM21 is not yet fully understood, but it involves binding of TRIM21 to the Fc-region of an antibody and subsequent autoubiquitination of TRIM21. In a Trim-Away experiment, the high affinity of TRIM21 to the Fc-region of an antibody is exploited to target endogenous proteins for degradation. An antibody against the target protein is introduced, and TRIM21 binds to the antibody-bound target protein and triggers the proteasomemediated degradation of the antibody-antigen complex together with TRIM21 (refs. ${ }^{20,21}$ ). TrimAway has enabled us to degrade a wide variety of proteins within minutes of application in different cell types ${ }^{15}$. For instance, we could acutely deplete Rec 8 in unmodified mouse oocytes ${ }^{15}$, an experiment that required complex genetics in the past ${ }^{22}$. We have also been able to deplete the intracellular signaling molecule NLRP3 in human primary macrophages, which was not possible in the past with nucleic acid-based depletion techniques ${ }^{15}$. Here, we (i) describe the design of TrimAway experiments; (ii) detail the selection and preparation of reagents for Trim-Away experiments; (iii) discuss the procedures for use of Trim-Away in mouse oocytes and pre-implantation embryos; (iv) present the procedures for use of Trim-Away in primary cells and cell lines; and (v) propose quality controls for Trim-Away experiments. We also highlight and give advice on critical steps in the Procedure.

\section{Overview of the procedure}

For a successful Trim-Away experiment, a specific antibody that targets an intracellular protein of interest must be delivered into cells that contain TRIM21. If the endogenous levels of TRIM21 are not sufficient for protein degradation, TRIM21 must also be introduced together with or before antibody delivery. In this protocol, we outline different methods for introducing antibody and TRIM21 into cells and give advice on how to preselect specific antibodies for a successful Trim-Away experiment. A routine Trim-Away experiment involves the following three events (Fig. 1):

1 Ensuring sufficient TRIM21 levels in target cells of interest. TRIM21 is widely expressed in different cell types $^{23}$. Depending on the expression level of the target protein, endogenous TRIM21 levels may be sufficient for Trim-Away ${ }^{15}$. But as TRIM21 is continuously degraded during a Trim-Away experiment and typically not expressed at high levels, the endogenous level may be insufficient for complete target protein degradation. Additional TRIM21 can be introduced in the form of a transgene (i.e., DNA), mRNA or protein. Using TRIM21-encoding DNA, we have transiently transfected cell lines or created stable lines with a TRIM21 expression cassette integrated into their genomes $^{15}$ (see also Box 1). Stable lines simplify the workflow in a Trim-Away experiment because only the delivery of the antibody is required for acute protein degradation. In certain cases, cells with low expression levels of TRIM21 must be preselected by FACS because high expression of TRIM21 can sometimes lead to the formation of protein aggregates, which are nonfunctional ${ }^{15}$. We have also used in vitro-transcribed mRNAs to transiently express TRIM21 in mouse oocytes ${ }^{15}$. Using mRNAs accelerates the expression of TRIM21 and allows fine-tuning of the expression level, 


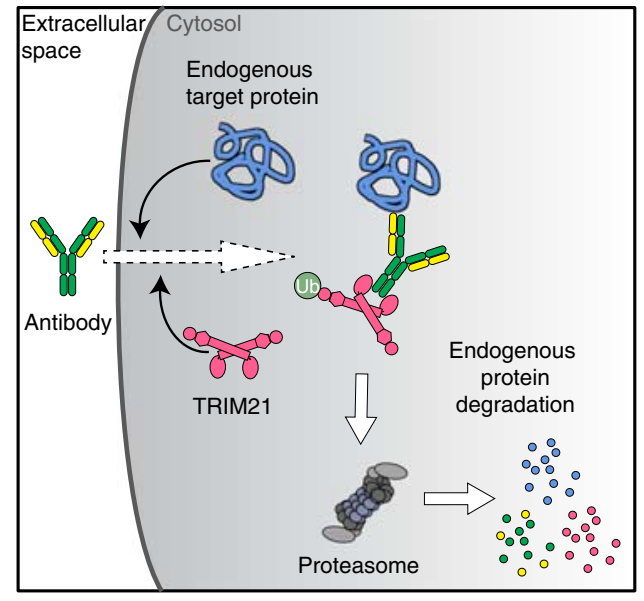

Fig. 1 | Schematic of the principle of Trim-Away.

but translation rates may vary for cells at different cell cycle stages. TRIM21 can also be introduced in the form of a recombinant protein, for instance, by electroporation ${ }^{15}$. Recombinant TRIM21 (see also Box 2) eliminates the incubation time that is required for protein expression from DNA or mRNA before a Trim-Away experiment. However, recombinant TRIM21 can be exhausted if not replenished.

2 Delivery of antibody. Depending on the experimental setup, the antibody can be delivered by either microinjection (Step 16) or electroporation (Steps 17-32). We have microinjected antibody into mouse oocytes and single adherent cells ${ }^{15}$. Microinjection allows tight control over the amount of antibody delivered into each cell and is the method of choice when individual cells must be analyzed rapidly upon triggering protein degradation. For bulk cell populations, we recommend delivering the antibody by electroporation using the Neon Transfection System ${ }^{15}$. Unlike protein transfection reagents, electroporation can be used to simultaneously deliver the antibody into the cytoplasm of many cells without antibody accumulation in the endosome and/or lysosome. In addition to conventional antibodies, we have also used a fusion between a nanobody and the Fc-region of human IgG1 for protein degradation by Trim-Away ${ }^{15}$. We have introduced this nanobody-Fc fusion into mouse oocytes as mRNA, but delivery as DNA or protein into other cell types should work as well ${ }^{15}$.

3 Target protein degradation. When the antibody has bound to the target protein, TRIM21 targets the antibody-antigen complex for degradation via the ubiquitin-proteasome pathway ${ }^{20,21}$. Degradation typically starts within 5-10 min after application of the antibody ${ }^{15}$. Complete depletion can be achieved within $\sim 3 \mathrm{~h}$, depending on the abundance and accessibility of the target protein, as well as the amount of antibody that was introduced into the cell ${ }^{15}$.

\section{Applications of the method}

Trim-Away uses TRIM21 to degrade endogenous proteins that are bound to an antibody. Our results suggest that any protein within a cell that is accessible by an antibody can be degraded by Trim-Away. Because TRIM21 proteins and antibodies are highly conserved among different mammalian species, Trim-Away works with a wide range of cells and antibodies ${ }^{24}$. Trim-Away acts very rapidly and does not require prior modification of the target protein. It also works in various cell types, including nondividing primary cells. Trim-Away also allows loss-of-function studies in primary immune cells, which could not be studied efficiently with nucleic acid-based depletion methods in the past.

Some specific examples of what can be studied are (i) the function of a protein within a defined cell cycle stage without affecting its other potential functions during the other cell cycle stages; (ii) the roles of proteins for the maintenance of cellular structures, even if these proteins are essential for the formation of these structures; (iii) the functions of the cytoplasmic pool of certain proteins; (iv) the functions of certain proteins with a particular post-translational modification; (v) the functions of specific isoforms of certain proteins; and (vi) structure-function relationships in certain proteins by depletion followed by rescue with different mutants. 


\section{Box 1 | Preparation of TRIM21 stable cell lines Timing 2-3 weeks}

Although TRIM21 is nearly universally expressed, its expression level varies between cell types. Because TRIM21 is degraded alongside the antibody and target antigen during Trim-Away, levels of endogenous TRIM21 may be insufficient to elicit complete degradation of the target antigen, especially in cases in which the target antigen is abundant. This can be remedied by the constitutive expression of TRIM21 stably integrated into the target cell genome. Here, we describe a protocol for stable cell line generation by transduction with pseudotyped lentiviral particles. However, alternative approaches for stable cell line generation can also be used.

\section{Reagents}

- DMEM (high glucose, GlutaMAX; Gibco, cat. no. 31966)

- FBS (Gibco, cat. no. 10270)

- DPBS (no calcium, no magnesium; Gibco, cat. no. 14190)

- Trypsin-EDTA (0.05\% (wt/vol), phenol red; Gibco, cat. no. 25300)

- Opti-MEM I reduced serum medium (Gibco, cat. no. 31985)

- psPAX2 (Addgene, plasmid no. 12260)

- pSMPP-mCherry-hTRIM21 (Addgene, plasmid no. 104972)

- pSMPP-mCherry-mTrim21 (Addgene, plasmid no. 104971)

- pMD2.G (Addgene, plasmid no. 12259)

- FuGENE 6 transfection reagent (Promega, cat. no. E2691)

- Polybrene (Santa Cruz Biotechnology, cat. no. sc-134220)

- Puromycin dihydrochloride (Gibco, cat. no. A1113803)

\section{Equipment}

- Corning tissue culture-treated culture dishes (100 mm × 20 mm; Sigma-Aldrich, cat. no. CLS430167)

-10-ml Syringe (BD Plastipak, cat. no. 302188)

- 33-mm Ezee syringe filters (0.45 $\mu \mathrm{m}$; PVDF; sterile; Elkay, cat. no. E25-PV45-50S)

- Corning Costar TC-treated multiple-well plates (six wells; clear; polystyrene plate; flat bottom; Sigma-Aldrich, cat. no. CLS3516-50E)

- Corning Costar TC-treated multiple-well plates (24 wells; flat bottom; Sigma-Aldrich, cat. no. CLS3527)

\section{Reagent setup}

- DMEM supplemented with $1 \times$ GlutaMAX and 10\% (vol/vol)FBS. Add $55 \mathrm{ml}$ of FBS to $500 \mathrm{ml}$ of DMEM. Store at $4{ }^{\circ} \mathrm{C}$ for up to 1 year from the date of manufacture until use.

\section{Procedure}

! CAUTION Ensure that virus work takes place at an appropriate level of biosafety according to national and institutional regulations.

1 Seed $2.5 \times 10^{6}$ HEK293T cells in a $10-\mathrm{cm}$ dish containing $10 \mathrm{ml}$ of DMEM supplemented with $1 \times$ GlutaMAX and $10 \%$ (vol/vol) FBS.

2 Incubate the cells for $16-24 \mathrm{~h}$ at $37{ }^{\circ} \mathrm{C}$ in a $5 \% \mathrm{CO}_{2}$ atmosphere.

3 In a sterile 1.5-ml reaction tube, prepare $200 \mu$ l of Opti-MEM I, $2 \mu$ g of HIV GagPol expression plasmid (e.g., psPAX2), $2 \mu g$ of TRIM21 construct in lentiviral transfer vector (e.g., pSMPP-mCherry-hTRIM21) and $1 \mu \mathrm{g}$ of VSV-G glycoprotein expression plasmid (e.g., pMD2.G).

4 Mix the plasmids well by gentle vortexing and bring to the bottom of the tube with a brief spin in a microcentrifuge $\left(2,000 \mathrm{~g}, 25^{\circ} \mathrm{C}, 5 \mathrm{~s}\right)$.

5 Add $12 \mu$ of FuGENE 6 transfection reagent to the plasmids and mix immediately by flicking. Bring the liquid to the bottom of the tube with a brief spin in a microcentrifuge $\left(2,000 \mathrm{~g}, 25^{\circ} \mathrm{C}, 5 \mathrm{~s}\right)$.

6 Incubate the transfection mix for 20 min at room temperature.

7 Add the transfection mix dropwise to the center of the dish of HEK293T cells with gentle swirling.

$\triangle$ CRITICAL STEP Mixing by pipetting or vigorous agitation should be avoided, as it may dislodge HEK293T cells from tissue culture dishes.

8 Incubate the cells for $16-24 \mathrm{~h}$ at $37^{\circ} \mathrm{C}$ in a $5 \% \mathrm{CO}_{2}$ atmosphere.

9 Gently remove the medium and replace with fresh, prewarmed DMEM supplemented with 1× GlutaMAX and 10\% (vol/vol) FBS, and then return the cells to the incubator for a further $48 \mathrm{~h}$.

10 (Optional) Examine the cells under a fluorescence microscope to ensure high levels of mCherry-hTRIM21 expression in virus-producing cells resulting from transfection and single-cycle re-infection.

11 Harvest the supernatant directly into a $10-\mathrm{ml}$ syringe; then filter at $0.45 \mu \mathrm{m}$, separate into $1-\mathrm{ml}$ aliquots and store the virus particles at $-80{ }^{\circ} \mathrm{C}$. PAUSE POINT Viral particles can be stored indefinitely at $-80^{\circ} \mathrm{C}$.

12 Seed adherent target cells at $1 \times 10^{5}$ per well in six-well plates in appropriate medium. Suspension cells can be seeded in 24 -well plates in their normal medium with polybrene at $10 \mu \mathrm{g} / \mathrm{ml}$ and transduced immediately (step 15).

13 Incubate the cells for $16-24 \mathrm{~h}$ at $37^{\circ} \mathrm{C}$ in a $5 \% \mathrm{CO}_{2}$ atmosphere.

14 Replace the medium with fresh medium containing $10 \mu \mathrm{g} / \mathrm{ml}$ polybrene ( $2 \times$ final concentration).

15 Thaw the virus supernatant at room temperature.

16 On the first transduction of a new cell type, or after production of a new batch of virus supernatant, add virus at a range of concentrations to determine the optimal level for low-multiplicity transduction $(<0.1$ transducing particles per cell). Prepare a five-fold serial dilution of virus in medium such that successive wells will receive 1, 5, 25, 125 and $625 \mu \mathrm{l}$ of virus. Add virus to cells in a volume equal to that of the plating medium such that the final polybrene concentration is $5 \mu \mathrm{g} / \mathrm{ml}$. Include a control well that does not receive virus.

17 Incubate the cells for $48 \mathrm{~h}$ at $37{ }^{\circ} \mathrm{C}$ in a $5 \% \mathrm{CO}_{2}$ atmosphere.

18 Analyze for transgene expression by fluorescence microscopy or flow cytometry. Select conditions in which $<10 \%$ of cells are expressing mCherry-TRIM21, in order to minimize the number of multiply transduced cells.

19 Add puromycin to the cells at an appropriate concentration for the cell type, typically $0.5-5 \mu \mathrm{g} / \mathrm{ml}$.

20 After 1 week of selection, all untransduced cells and the untreated control well should be killed. Expand the cell population for freezing and for use in Trim-Away experiments.

$\triangle$ CRITICAL STEP Regularly ensure that the distribution of mCherry-TRIM21 is diffuse and cytoplasmic by fluorescence microscopy (Fig. 4b,e). Large crescent-shaped aggregates of mCherry-TRIM21 that are nonfunctional can form if levels of expression are too high or cells become overconfluent. This seems to be a cell-type-specific phenomenon and occurs in cell types in which expression from viral promoters is particularly high, for instance, in HEK293 cells. In such a case, consider FACS-sorting low mCherry-positive cells or deriving clonal lines and selecting lowexpressing colonies. Alternatively, consider using a tetracycline-inducible promoter to drive TRIM21 expression. 


\section{Box 2 | Preparation of recombinant TRIM21 protein (3 d)}

This box describes the procedure for expression and purification of full-length human TRIM21 protein His-Lipoyl-hTRIM21 (Fig. $2 \mathrm{~g}$ ). His-LipoylhTRIM21 is expressed from a T7-promoter-driven bacterial expression plasmid HLTV-hTRIM21. HLTV-hTRIM21 is available from Addgene (Reagents) and is a low copy-number ampicillin-resistant plasmid with an $\mathrm{N}$-terminal His tag that can be optionally cleaved by TEV protease.

\section{Biological materials}

- OverExpress C41(DE3) chemically competent cells (Sigma-Aldrich, cat. no. CMC0017)

\section{Reagents}

- HLTV-hTRIM21 (Addgene, plasmid no. 104973)

- LB-Agar Miller (Formedium, cat. no. LMM02)

- 2x TY medium (Formedium, cat. no. YDB1L)

- $\mathrm{D}-(+)$-Glucose (Sigma-Aldrich, cat. no. G8270)

- Ampicillin sodium salt (Melford, cat. no. A0104)

- IPTG (Melford, cat. no. MB1008)

- Bugbuster plus benzonase (Merck Millipore, cat. no. 70750-3)

- cOmplete, EDTA-free Protease Inhibitor Cocktail (Sigma-Aldrich, cat. no. 04693132001)

- Trizma base (Sigma-Aldrich, cat. no. T1503)

- Sodium chloride (Sigma-Aldrich, cat. no. S3014)

- Ni-NTA agarose (Qiagen, cat. no. 30210)

- Imidazole (Sigma-Aldrich, cat. no. 15513)

\section{Equipment}

- Poly-Prep chromatography column (Bio-Rad, cat. no 7311550)

- ÄKTA pure (GE Healthcare Life Sciences)

- S200 gel filtration column (GE Healthcare Life Sciences, cat. no. 28989336)

\section{Reagent setup}

- $100 \mathrm{mg} / \mathrm{ml}$ ampicillin. Add $10 \mathrm{~g}$ of ampicillin sodium salt to $100 \mathrm{ml}$ of sterile water. Filter at $0.45 \mu \mathrm{m}$. Divide into 10 -ml aliquots and store at $-20^{\circ} \mathrm{C}$ (for up to 1 year) until use.

- LB agar plates supplemented with $100 \mu \mathrm{g} / \mathrm{ml}$ ampicillin. Add $40 \mathrm{~g}$ of LB-Agar Miller to $1 \mathrm{~L}$ of sterile water. Autoclave at $120^{\circ} \mathrm{C}$ for $15 \mathrm{~min}$. Cool down briefly and add $1 \mathrm{ml}$ of $100 \mathrm{mg} / \mathrm{ml}$ ampicillin before pouring plates. Store at $4{ }^{\circ} \mathrm{C}$ (for up to 3 months) until use.

- $2 \times$ TY medium. Add $31 \mathrm{~g}$ of $2 \times$ TY medium to $1 \mathrm{~L}$ of sterile water. Autoclave at $120{ }^{\circ} \mathrm{C}$ for $15 \mathrm{~min}$. Freshly prepare before use.

- $0.1 \mathrm{M}$ IPTG. Add $2.38 \mathrm{~g}$ of IPTG to $100 \mathrm{ml}$ of sterile water. Filter at $0.45 \mu \mathrm{m}$. Divide into 10 -ml aliquots and store at $-20^{\circ} \mathrm{C}$ (for up to $1 \mathrm{year}$ ) until use.

- $10 \%$ (wt/vol) glucose. Add $10 \mathrm{~g}$ of glucose to $100 \mathrm{ml}$ of sterile water. Autoclave at $120{ }^{\circ} \mathrm{C}$ for $15 \mathrm{~min}$. Freshly prepare before use.

- $1 \mathrm{M}$ Tris ( $\mathrm{pH} \mathrm{8.0)}$ ). Add $12.1 \mathrm{~g}$ of Trizma base to $90 \mathrm{ml}$ of sterile water. Adjust the $\mathrm{pH}$ to 8.0 and bring the final volume to $100 \mathrm{ml}$ with sterile water. Store at room temperature (for up to 1 year) until use.

- $5 \mathrm{M} \mathrm{NaCl}$. Add $29.2 \mathrm{~g}$ of sodium chloride to $90 \mathrm{ml}$ of sterile water. Bring the final volume to $100 \mathrm{ml}$ with sterile water. Store at room temperature (for up to 1 year) until use.

- PBS (pH 8.0). Add $5 \mathrm{~g}$ of PBS tablet to $450 \mathrm{ml}$ of sterile water. Adjust the pH to 8.0 and bring the final volume to $100 \mathrm{ml}$ with sterile water. Store at room temperature (for up to 1 year) until use.

- $3 \mathrm{M}$ imidazole. Add $102.1 \mathrm{~g}$ of imidazole to $450 \mathrm{ml}$ of sterile water. Adjust the $\mathrm{pH}$ to 8.0 and bring the final volume to $500 \mathrm{ml}$ with sterile water. Store at room temperature (for up to 1 year) until use.

- Wash buffer (20 mM Tris (pH 8.0)-200 mM NaCl-10 mM imidazole). Add $1.7 \mathrm{ml}$ of $3 \mathrm{M}$ imidazole, $10 \mathrm{ml}$ of $1 \mathrm{M}$ Tris (pH 8.0) and $20 \mathrm{ml}$ of $5 \mathrm{M}$ $\mathrm{NaCl}$ to $468.3 \mathrm{ml}$ of sterile water. Store at room temperature (for up to 1 year) until use.

- $20 \mathrm{mM}$ Tris ( $\mathrm{pH} \mathrm{8.0)}-200 \mathrm{mM} \mathrm{NaCl}$. Add $10 \mathrm{ml}$ of $1 \mathrm{M}$ Tris ( $\mathrm{pH} \mathrm{8.0)}$ ) and $20 \mathrm{ml}$ of $5 \mathrm{M} \mathrm{NaCl}$ to $470 \mathrm{ml}$ of sterile water. Store at room temperature (for up to 1 year) until use.

\section{Equipment setup}

- ÄKTA pure. The HiLoad 26/600 Superdex 200 pg column should be set up according to the manufacturer's instructions.

\section{Procedure}

1 Freshly transform C41(DE3) cells with HLTV-hTRIM21, and plate the transformed cells onto a LB-agar plate supplemented with $100 \mu \mathrm{gg} / \mathrm{ml}$ ampicillin overnight at $37^{\circ} \mathrm{C}$.

2 Pick colonies and inoculate overnight cultures in $25 \mathrm{ml}$ of $2 \times$ TY supplemented with $2 \%$ (wt/vol) glucose and $100 \mu \mathrm{gg} / \mathrm{ml}$ ampicillin. Shake at 220 r.p.m. at $37^{\circ} \mathrm{C}$

3 Inoculate 1 liter of $2 \times$ TY medium with $10 \mathrm{ml}$ of overnight culture, and supplement with $0.1 \%$ (wt/vol) glucose and $100 \mu \mathrm{g} / \mathrm{ml}$ ampicillin. Shake at 220 r.p.m. at $37^{\circ} \mathrm{C}$.

4 At $\mathrm{OD}_{600}$ 0.8-1.2 (after $\sim 3 \mathrm{~h}$ ), induce with $0.1 \mathrm{mM}$ IPTG and reduce the temperature to $22^{\circ} \mathrm{C}$.

$\triangle$ CRITICAL STEP Induction at OD values $>1.2$ or overnight growth at higher temperatures will markedly reduce yields.

5 The next day, harvest the cells in 1-liter centrifuge bottles by centrifuging at 5,000g for 20 min at $4{ }^{\circ} \mathrm{C}$ (e.g., using a RC3B rotor). Discard the medium.

DAUSE POINT Bacterial pellets can be frozen at $-20^{\circ} \mathrm{C}$ and stored for up to 1 week, and protein preparation can be continued later.

6 Resuspend the cells in $20 \mathrm{ml}$ of Bugbuster plus 1/2 of a crushed Complete, EDTA-free Protease Inhibitor Cocktail tablet per centrifuge tube. Add $1 \mathrm{M}$ Tris ( $\mathrm{pH} 8.0$ ) to a final concentration of $20 \mathrm{mM}$ and $5 \mathrm{M} \mathrm{NaCl}$ to $200 \mathrm{mM}$.

7 Keep the cells in $50-\mathrm{ml}$ centrifuge tubes ( $40 \mathrm{ml}$ per tube) on ice-water. Sonicate for a minimum of 2 min with 10 -s pulses followed by $10 \mathrm{~s}$ of rest.

$\triangle$ CRITICAL STEP Sonicated solution should be carefully monitored so that it does not become warm. Successful sonication is indicated by a noticeable reduction in the viscosity of the solution. 


\section{Box 2| (continued)}

8 Spin down in a SS34 rotor at 20,000 g for 40 min at $4{ }^{\circ} \mathrm{C}$. Carefully decant the supernatant. Proceed to Step 9 during centrifugation.

9 In the meantime, wash $10 \mathrm{ml}$ of Ni-NTA agarose in $50 \mathrm{ml}$ of PBS (pH 8.0). Spin at 2,000g for 3 min at room temperature and discard the supernatant. Repeat once.

$\triangle$ CRITICAL STEP Centrifugation of Ni-NTA beads at higher speeds for longer time periods will compress the resin and reduce its binding capacity.

10 Add Ni-NTA agarose to the cleared supernatant from step 8 in centrifuge tubes, supplement with 10 mM imidazole and rotate to allow mixing at $4{ }^{\circ} \mathrm{C}$ for $\sim \mathrm{h}$.

11 Spin down the resin at 2,000g for $3 \mathrm{~min}$ at $4{ }^{\circ} \mathrm{C}$ and discard the supernatant.

12 Resuspend the resin in $40 \mathrm{ml}$ of wash buffer, re-spin and discard the supernatant. Repeat this step once.

13 Decant the washed resin into an empty gravity-flow column. Wash the resin in place with two bed volumes of wash buffer.

14 Elute the protein from the resin with wash buffer supplemented with $300 \mathrm{mM}$ imidazole.

15 Analyze the eluted protein by SDS-PAGE to ensure that the purification has been successful. This should be visible as a single band running at $\sim 66 \mathrm{kDa}$ following Coomassie staining (Fig. 2g). If so, proceed to step 16 .

16 Apply the eluted protein to an S200 gel filtration column equilibrated with $20 \mathrm{mM}$ Tris (pH 8.0 ) $-200 \mathrm{mM} \mathrm{NaCl}$. Select the size of the S200 column depending on the volume of eluate and total amount of protein to be purified. Follow the manufacturer's guidelines for column capacity and flow rate.

$\triangle$ CRITICAL STEP Eluted protein will not remain stable under a high concentration of imidazole for prolonged periods, so it must be immediately buffer-exchanged, desalted or gel-filtered into $20 \mathrm{mM}$ Tris ( $\mathrm{pH} 8.0$ ) $-200 \mathrm{mM} \mathrm{NaCl}$.

$\triangle$ CRITICAL STEP Soluble aggregated materials will elute in the void volume of the column and should be discarded.

17 Analyze the peak fractions by SDS-PAGE to ensure that the correct peak is collected and to determine which fractions should be combined. $\triangle$ CRITICAL STEP TRIM21 is an elongated molecule with a large Stokes radius; if molecular weight standards are used to guide fraction collection, then note that TRIM21 will behave as if it is a trimer (i.e., $3 \times$ its expected molecular weight ( 165 kDa)) in an S200 gel filtration column.

18 Concentrate the combined fractions of TRIM21 using 50-kDa cutoff centrifugal filter units, prepare aliquots and snap-freeze them in liquid nitrogen.

$\triangle$ CRITICAL STEP The protein solution should be carefully monitored during centrifugation and regularly mixed to prevent superconcentration at the cellulose membrane.

DAUSE POINT The purified protein can be stored at $-80^{\circ} \mathrm{C}$ as long as no signs of protein degradation can be seen.

\section{Advantages and limitations of the method}

In comparison with other currently available protein depletion techniques, Trim-Away has several unique advantages. First, Trim-Away acts directly at the protein level. It is therefore effective against long-lived proteins, and the development of phenotypes does not rely on inherent turnover of the target protein. Second, Trim-Away does not require modification of the genome. It therefore greatly facilitates loss-of-function studies in models for which DNA- and RNA-based depletion methods are often ineffective, including nondividing primary cells. Third, Trim-Away is a protein-based depletion method and can be used in primary immune cells that have active nucleic acid-sensing machineries. Fourth, TRIM21 is widely expressed in most tissues. This means that it may often be possible to take advantage of endogenously produced protein and not rely on ectopic overexpression or introduction of recombinant protein. Fifth, TRIM21 does not have a cellular housekeeping function, in contrast to E3s such as von Hippel-Lindau that are typically used in PROTAC applications ${ }^{25}$. This means there is no competition for endogenous substrates and less likelihood that normal metabolism will be impacted. Last, Trim-Away uses off-the-shelf reagents for protein targeting. Antibodies have been produced for almost all proteins and are widely commercially available.

As with any technique, Trim-Away has limitations. One limitation is that Trim-Away depends on the intrinsic proteasome activity. Hence, the efficiency of Trim-Away may be compromised in certain cell types and/or at certain cell cycle stages accordingly. Because Trim-Away is a protein-targeting method, another limitation is that it is more challenging to evaluate off-targets as compared with gene- or mRNA-targeting methods. Thus, antibodies used for protein-targeting must be carefully selected and validated before use, as discussed in the 'Experimental design' section ('Antibody selection'). In addition, an antigen buried within the native protein or a target stably localized in a particular cellular compartment such as the nucleus will not be accessible for degradation by the antibody. But the latter case may be circumvented by the use of a smaller nanobody-Fc fusion ${ }^{15}$. Finally, the usefulness of Trim-Away may be limited in certain models such as intact tissues, in which it is challenging to deliver antibody by microinjection or electroporation. However, as there are intensive efforts to develop cell delivery reagents, it may become possible in the future to use TrimAway even in intact-tissue applications. An important potential limitation in Trim-Away is that TRIM21 both targets proteins for degradation and can activate innate immune signal transduction pathways such as NF-KB (ref. ${ }^{26}$ ). This is because TRIM21 synthesizes K63-ubiquitin chains, which when released by the proteasomal DUB RPN11 act as immune second messengers ${ }^{21}$. Fortunately, 
there is a high activation barrier for TRIM21 signaling, and NF- $\kappa \mathrm{B}$ activation is highly sensitive to changes in antibody affinity, concentration and viral dose ${ }^{27,28}$. For instance, in our Trim-Away experiment targeting I $\mathrm{KB} \alpha$ for degradation, NF- $\kappa \mathrm{B}$ was only weakly activated in control cells in which $\mathrm{mTOR}_{\text {was depleted }}{ }^{15}$. If activation of NF- $\kappa \mathrm{B}$ is a concern during Trim-Away, this can be assayed for either through the use of an NF- $\kappa \mathrm{B}$-luciferase reporter or by qPCR for cytokine transcripts.

\section{Experimental design Antibody selection}

Similar to other antibody-based techniques, the specificity of the antibody is critical for a successful Trim-Away experiment. A good antibody should degrade all antigens bearing the targeted epitope (i.e., on-target) but not irrelevant antigens (i.e., off-target). When selecting an antibody for TrimAway, several parameters should be considered:

- Antibody species. The TRIM21-antibody interaction is highly conserved and maintained both within and between murine, canine, primate and human species ${ }^{24}$. Our preliminary results with anti-GFP antibodies raised in mouse, rabbit and goat suggest no substantial difference in their Trim-Away efficiency at the same concentration. We have also successfully used antibodies raised in rat $^{15}$. However, antibodies from less common hosts such as guinea pig and sheep should be tested before actual application. TRIM21 does not bind to chicken antibodies.

- Isotype of antibody. TRIM21 has the highest affinity to IgG, followed by IgM and IgA ${ }^{28}$. Binding of TRIM21 to IgD or IgE remains unknown. TRIM21 does not bind to IgY.

- Form of antibody. Commercial antibodies are offered in different forms such as full-length Ig, Fab and Fab2. Because TRIM21 binds to the Fc-region of antibodies, Fab and Fab2 should be avoided. Considering the growing supply of nanobodies, we have also successfully used a fusion between a nanobody and the Fc-region of human IgG1 for Trim-Away experiments ${ }^{15}$.

- Clonality of antibody. We have had success with both polyclonal and monoclonal antibodies. Because of lot variations between polyclonal antibodies, we recommend using monoclonal antibodies because they may yield more reproducible results.

- Antigen of antibody. The epitope should be accessible to the antibody under native conditions.

- Antibodies against post-translationally modified proteins. Our preliminary results suggest that TrimAway can also be used to selectively degrade proteins with post-translational modifications, as long as specific antibodies are available. However, these antibodies must be evaluated particularly carefully before use. For instance, we observed nonspecific binding of some polyclonal antibodies against phosphorylated kinetochore proteins to acentriolar microtubule-organizing centers, which are also enriched in phosphorylated proteins in mouse oocytes (and vice versa).

\section{Antibody validation}

Before proceeding to Trim-Away, we generally validate the antibody by performing at least two of the experiments listed below:

- Co-localization with the fluorescently tagged protein. Most proteins localize correctly when they are tagged with a fluorescent protein. To evaluate whether an antibody is specific, we compare the localization of the fluorescent protein with the pattern obtained by immunofluorescence using the antibody of interest. They should be identical. Exceptions to this are some long-lived proteins such as Rec 8 and CenpA in mouse oocytes ${ }^{22,29}$. These proteins are loaded correctly only during particular stages of development, which do not allow further incorporation or displacement by their fluorescent reporter afterward. In addition, comparison of the staining pattern with published data from other cell types is a useful way to test for specificity.

- Antibody delivery followed by immunofluorescence. To determine the ability of an antibody to recognize the target protein in live cells, we deliver the antibody by either microinjection or electroporation, and fix and stain with a secondary antibody. The delivered antibody should show the same localization pattern as the immunofluorescence of the target protein using a different antibody. It is also possible to fluorescently label the antibody in order to observe correct binding to the target protein in live cells ${ }^{30}$. However, in some cases, antibody delivery may cause target protein degradation via endogenous TRIM21 and/or cause target protein mislocalization due to the inhibitory effects of antibody binding. We therefore recommend traditional immunofluorescence as the preferred method to validate antibodies.

- Immunoprecipitation followed by western blotting. To determine the ability of an antibody to recognize the target protein in native conformation in cells, we perform immunoprecipitation on the whole-cell 


\section{Box 3 | Peptide pre-incubation assay Timing 2-4 d}

This box describes the procedures for pre-incubating a primary antibody with its immunizing peptide before immunofluorescence. This assay allows the evaluation of nonspecific binding of the antibody under native conditions.

\section{Reagents}

- Paraformaldehyde (4\% (wt/vol) in PBS; VWR, cat. no. J61899)

- Immunizing peptide (lyophilized; from solid-phase peptide synthesis by a company such as GenScript; custom order)

- BSA solution (30\% (wt/vol) in DPBS; Sigma-Aldrich, cat. no. A9576)

- PBS tablets (Gibco, cat. no. 18912)

- Triton X-100 solution (10\% (vol/vol) in $\mathrm{H}_{2} \mathrm{O}$; Sigma-Aldrich, cat. no. 93443)

\section{Reagent setup}

PBT. Add $1 \mathrm{~g}$ of PBS tablets and $5 \mathrm{ml}$ of $10 \%$ (vol/vol) Triton X-100 solution to $95 \mathrm{ml}$ of water. Store at $4{ }^{\circ} \mathrm{C}$ until use (for up to 1 year).

PBT-BSA. Add $1 \mathrm{~g}$ of PBS tablets, $5 \mathrm{ml}$ of $10 \%$ (vol/vol) Triton X-100 solution and $10 \mathrm{ml}$ of $30 \%$ (wt/vol) BSA solution to $85 \mathrm{ml}$ of water. Store at $4{ }^{\circ} \mathrm{C}$ until use (for up to 1 year).

\section{Procedure}

1 Add an appropriate volume of solvent to dissolve the lyophilized peptide to a final concentration of $133 \mu \mathrm{M}$.

$\triangle$ CRITICAL STEP Recombinant protein has been used with success as well, albeit at a higher cost.

2 Fix the identical samples with $4 \%$ (wt/vol) paraformaldehyde in PBS for $15-30$ min at $37^{\circ} \mathrm{C}$.

3 Permeabilize the samples in PBT overnight at $4{ }^{\circ} \mathrm{C}$.

4 Block the samples in PBT-BSA overnight at $4{ }^{\circ} \mathrm{C}$.

$5 \mathrm{Mix}$ the components in the table below and incubate at $4{ }^{\circ} \mathrm{C}$ overnight.

\begin{tabular}{lll} 
Component & Amount per reaction $(\mu \mathrm{l})$ & Final concentration \\
\hline $1 \mathrm{mg} / \mathrm{ml}$ (primary) antibody & 0.2 & $10 \mu \mathrm{g} / \mathrm{ml}(66.7 \mathrm{nM})$ \\
$133 \mu \mathrm{M}$ peptide & $\begin{array}{l}0 \text { (for no-peptide control) or 2 (for } \\
\text { pre-incubated peptide sample) }\end{array}$ & 0 or $13.3 \mu \mathrm{M}(200 \times 66.7 \mathrm{nM})$ \\
PBT-BSA & To 20 &
\end{tabular}

6 Pre-clear the mixes from step 5 by centrifuging at $20,000 \mathrm{~g}$ for 5 min at $4{ }^{\circ} \mathrm{C}$.

7 Incubate one sample with the precleared mix without peptide from step 4 (i.e., no-peptide control) and the other sample with the precleared mix with peptide from step 4 (i.e., peptide pre-incubated sample) overnight at $4{ }^{\circ} \mathrm{C}$.

8 Wash the samples in PBT-BSA for 5 min at room temperature $\left(25^{\circ} \mathrm{C}\right)$ three times.

9 Incubate the samples with secondary antibodies diluted in PBT-BSA for $1 \mathrm{~h}$ at room temperature.

10 Wash the samples three times in PBT for 5 min at room temperature.

11 Image the samples in PBS.

12 Assess nonspecific bindings from the signal present in both the no-peptide control and peptide pre-incubated sample.

lysate in non-denaturing conditions. The specific antibody should enrich a single band corresponding to the target protein over background band(s) (if any) in western blot. We do not recommend assessing the antibody by performing western blotting alone, as that provides information on only the specific binding of the antibody to the denatured epitope.

- Cross-validation with other approaches. To test for specific binding of an antibody, when possible, we first deplete the target protein with other approaches such as genome-editing or RNAi. This is followed by immunofluorescence using the antibody of interest. Specific binding of the antibody is confirmed by a reduction in the signal relative to the control. Such validation may also be provided by some antibody suppliers.

- Peptide pre-incubation followed by immunofluorescence. To test for nonspecific binding of an antibody, we pre-incubate the antibody with the immunizing peptide before immunofluorescence (Box 3). The degree of background binding can be estimated by the level of signal retained after blocking specific binding of the antibody. We highly recommend this assay for post-translational-modification-specific antibodies.

\section{Source of TRIM21}

TRIM21 is widely expressed in most tissues ${ }^{23}$. This means that most cell types will contain endogenous TRIM21 protein, and this may be sufficient for Trim-Away to work simply by delivering an 
antibody to unmodified cells. Indeed, we have found that RPE-1 cells and primary macrophages contain sufficient endogenous TRIM21 for Trim-Away of several different target proteins ${ }^{15}$. Cell types can be screened for TRIM21 expression levels by RT-PCR or by western blotting using antiTRIM21 antibodies (see the 'Reagents' section). The relative expression levels of TRIM21 can be compared with those of low-expressing cells (HEK293T) or high-expressing cells (RPE-1). As an initial Trim-Away experiment, we suggest delivering the antibody to the cells of interest and screening for target protein depletion. Depending on the cell type and relative expression levels of cellular TRIM21 and the target protein, antibody delivery alone may be sufficient for target protein depletion. If target protein depletion does not occur, or is incomplete, then methods for increasing cellular TRIM21 levels can be considered from among the following options:

- TRIM21 overexpression. In oocytes and early embryos, we found it necessary to overexpress TRIM21 for successful Trim-Away. In these cell types, microinjection of Trim $21 \mathrm{mRNA}$ is the recommended method. In bulk cell populations, it is important to ensure that all cells in the population overexpress TRIM21. To achieve this, we recommend generating stable cell lines as described in Box 1.

- Recombinant TRIM21 protein. For some cell types, particularly primary cells, it is not possible to generate stable cell lines overexpressing TRIM21. In this case, it may be preferable to co-deliver a recombinant TRIM21 protein together with an antibody. Recombinant TRIM21 protein can be readily produced from bacteria as described in Box 2. Bacterially produced proteins may contain bacterial contaminants that could perturb certain cell types, such as immune cells. In these cases, it may be preferable to purify TRIM21 from insect or mammalian cells.

Trim-Away by microinjection of oocytes and early embryos

In our initial study, we microinjected Trim 21 mRNA into arrested germinal vesicle (GV) oocytes, and incubated the oocytes for expression and recovery for $3 \mathrm{~h}$, followed by microinjection of the antibody ${ }^{15}$. Depending on the biological questions to be addressed, antibodies can be microinjected immediately before meiotic release or during meiosis. Although sequential injections can be mastered by scientists with extensive experience in micromanipulation, a significant reduction in oocyte survival and developmental rates is frequently observed for less experienced operators.

We have therefore developed a simplified protocol that involves single microinjection, which minimizes harm to the cells. Here, we present protocols for both hydraulic- and pressure-based microinjection systems. Hydraulic-based systems allow injection of a defined volume but require the use of mercury-filled needles ${ }^{31}$. Although it is challenging to consistently inject a defined volume using pressure-based systems, these systems are more widely available, do not involve mercury and allow more rapid microinjections. The new protocols offer several benefits. First, single microinjection improves survival and developmental rates to levels comparable to those of routine live-imaging experiments. Second, depletion starts immediately after microinjection as Trim 21 mRNA is translated, which can be advantageous for early embryos that cannot be arrested at a particular stage of development. Last, target proteins that are expressed at high endogenous levels (which can take up to 3-4 h for full depletion) are more completely degraded before meiotic release, and phenotypes during early meiosis are more consistent between individual oocytes.

In addition, we optimized our protocol for preparing mRNAs encoding Trim 21 and reporters for live-cell imaging with several modifications. First, the use of an anti-reverse cap analog (ARCA) yields transcripts that are all capped in the correct orientation at the $5^{\prime}$ end and, hence, have a higher translation efficiency than mRNAs that have been synthesized with a standard cap analog. Second, the increased ratio of cap analog and GTP relative to other NTPs enables the synthesis of transcripts of up to $12 \mathrm{~kb}$ at high yield. Finally, the incorporation of a column cleanup step removes all free nucleotides, allowing higher accuracy in determination of mRNA concentration and reproducibility in expression level.

Trim-Away by electroporation of primary cells and cell lines

A successful Trim-Away experiment in bulk cell populations depends on efficient delivery of antibody to all the cells in a population. Antibodies are large molecules $(\sim 150 \mathrm{kDa})$ that cannot cross cell membranes. We initially tested antibody delivery by conventional chemical- and lipid-based transfection reagents in addition to several different commercially available protein and antibody transfection reagents. However, this most often led to antibodies being trapped inside intracellular vesicles with very little antibody actually reaching the cytosol. We also tested classic electroporation using conventional cuvettes. However, this caused high levels of cell death, and surviving cells tended to have morphological changes and exhibit antibodies stuck to the cell surface, trapped inside 
intracellular vesicles, and aggregated inside the cells. We and others have found that a new type of electroporation device with a capillary electrode ${ }^{15,30,32,33}$, available commercially as the Neon Transfection System, can be used to deliver antibodies directly to the cytoplasm of almost $100 \%$ of cells without cell death. Cells are morphologically normal after electroporation with the Neon system and re-adhere and proliferate at a rate similar to that of non-electroporated cells ${ }^{15}$. Thus, Trim-Away experiments can be performed by electroporation of antibodies, and phenotypes can be analyzed as soon as the target protein is depleted, which is usually within $3 \mathrm{~h}$. In addition, we found that efficient antibody delivery can also be achieved in primary cells such as normal human lung fibroblasts, mouse bone-marrow-derived macrophages and human monocyte-derived macrophages ${ }^{15}$. Here, we include an optimized antibody electroporation protocol that we found to be suitable for antibody delivery in all cell types tested so far.

\section{Trim-Away controls}

To properly interpret a Trim-Away experiment, certain controls can be included. To control for antibody delivery, we recommend using isotype control antibodies of the same clonality as the antibodies used for targeted protein degradation. Control antibodies are often raised against proteins that are not normally expressed in mammalian cells, such as GFP or yeast proteins, and are readily available from most antibody suppliers. Additional controls such as delivery of antibody buffer alone and non-electroporated/microinjected cells can also be included. If a Trim-Away experiment requires TRIM21 overexpression, we also recommend including non-overexpressing cells as an additional control. Importantly, we found that both antibody delivery and TRIM21 overexpression did not perturb cell proliferation or induce major changes in global transcript levels ${ }^{15}$.

\section{Confirmation of protein depletion by Trim-Away}

Confirmation of protein depletion by Trim-Away can be achieved by standard methods used to detect cellular levels of endogenous proteins. For example, immunofluorescence, flow-cytometry or western blotting methods are suitable. When using secondary antibody-based detection methods to detect cellular levels of target proteins after treatment with Trim-Away (and also other nontargeted cellular proteins), it is important to take into account that secondary antibodies will also detect residual electroporated/microinjected antibody present in the cell if the secondary antibodies are raised against the same species of antibody as the delivered antibody. This problem will arise if the antibody delivered to cells for Trim-Away is also used as a primary antibody for protein detection. When performing western blotting, this will result in detection of IgG heavy chain and light chain on the membrane (at $\sim 50 \mathrm{kDa}$ and $\sim 25 \mathrm{kDa}$, respectively), which may interfere with detection of endogenous proteins. When performing immunofluorescence or flow cytometry, this will result in higher levels of background signal. In all detection methods, this problem can be avoided by using primary antibodies of a different species than the antibodies delivered to the cells for Trim-Away. If there is no option but to use the same antibody (or antibodies from the same species) for Trim-Away and for detection, HRP-conjugated secondary antibodies that bind only to the native non-denatured IgG can be used for western blotting, and primary antibodies can be directly labeled with fluorophores for immunofluorescence and flow cytometry.

\section{Confirmation of specificity in a Trim-Away experiment}

The specificity of depletion in a Trim-Away experiment is determined by the specificity of the antibody used. Thus, antibodies should be selected carefully, as described above (see 'Antibody selection'). If multiple antibodies raised against different regions of the same target protein are available, these can be used to confirm the specificity of a Trim-Away phenotype. Rescue experiments can also be performed by overexpressing exogenous target protein. When performing rescue experiments, it should be considered that the antibody delivered during Trim-Away will probably also target the exogenous protein for degradation. In this case, it may be necessary to increase expression levels of the exogenous protein in order to saturate the cellular antibody and TRIM21 to allow for a rescue. Similarly, the amount of delivered antibody should be minimized to aid rescue experiments. It may also be possible to rescue a phenotype by overexpressing exogenous protein from a different species that is not recognized by the antibody used for Trim-Away. Another way to confirm the specificity of depletion is to express a fluorescently tagged target protein. Upon acute delivery of the antibody against the target protein, the fluorescent signal should be reduced as a result of co-degradation of the fluorescent tag. 


\section{Biological materials}

- Mice (e.g., Charles River, cat. no. FVB/NCrl) ! CAUTION Animal experiments must be carried out according to institutional and national regulations. All experiments in this procedure involving live mice were approved by the MRC Cambridge Ethical Review Committee and the UK Home Office under project license nos. PPL 70/8087 and PPL PCF3F9520.

- Cell lines of interest. We have used 293 (HEK-293; ATCC, cat. no. CRL-1573), NIH/3T3 (ATCC, cat. no. CRL-1658), hTERT RPE-1 (ATCC, cat. no. CRL-4000), U-2 OS (ATCC, cat. no. HTB-96), HCT 116 (ATCC, cat. no. CCL-247) and HeLa (ATCC, cat. no. CCL-2) cells, although any mammalian cell line can be used. Cell lines should be grown in the appropriate growth media and maintained with standard cell culture methods. ! CAUTION Cell lines should be regularly checked to ensure that they are authentic and not infected with mycoplasma.

- Primary cells of interest. We have used NHLF (Lonza, cat. no. CC2512), human monocyte-derived macrophages (from patient blood samples, derived as described in ref. ${ }^{15}$ ) and mouse bone-marrowderived macrophages (derived as described in ref. ${ }^{15}$ ), although any mammalian primary cells may be used. ! CAUTION Cell lines should be regularly checked to ensure that they are authentic and not infected with mycoplasma.

\section{Reagents}

Antibody preparation

- NAb Protein A/G Spin Kit (0.2 ml; Thermo Fisher Scientific, cat. no. 89950)

- Water (for embryo transfer; Sigma-Aldrich, cat. no. W1503)

- Phosphate-buffered saline (PBS) tablets (Gibco, cat. no. 18912)

Isolation and culture of mouse oocytes and pre-implantation embryos

- Culture medium such as M2 medium (homemade or Sigma-Aldrich, cat. no. MR-015P)

- $\mathrm{NaCl}$ (Sigma-Aldrich, cat. no. S5886)

- KCl (Sigma-Aldrich, cat. no. P5405)

- $\mathrm{CaCl}_{2} \cdot 2 \mathrm{H}_{2} \mathrm{O}$ (Sigma-Aldrich, cat. no. C7902)

- $\mathrm{KH}_{2} \mathrm{PO}_{4}$ (Sigma-Aldrich, cat. no. P5655)

- $\mathrm{MgSO}_{4} \cdot 7 \mathrm{H}_{2} \mathrm{O}$ (Sigma-Aldrich, cat. no. 63138)

- $\mathrm{NaHCO}_{3}$ (Sigma-Aldrich, cat. no. S3817)

- HEPES (Gibco, cat. no. 15630)

- Na lactate (Sigma-Aldrich, cat. no. L7900)

- Na pyruvate (Sigma-Aldrich, cat. no. 11360)

- Glucose monohydrate (Sigma-Aldrich, cat. no. 49158)

- Penicillin G (Sigma-Aldrich, cat. no. P3032)

- Streptomycin (Sigma-Aldrich, cat. no. S9137)

- BSA (Sigma-Aldrich, cat. no. A3311)

- Phenol red (Sigma-Aldrich, cat. no. P3532)

- Oil (mineral: Sigma-Aldrich, cat. no. M5310, or paraffin: Merck Millipore, cat. no. 107174)

- Dibutyryl cyclic adenosine monophosphate (Sigma-Aldrich, cat. no. D0627-1G)

Constitutive Trim-Away in mouse oocytes and pre-implantation embryos

- Hydrochloric acid (36.5-38.0\% (vol/vol); Sigma-Aldrich, cat. no. H1758)

- Silicon grease (medium vacuum; Bayer; Sigma-Aldrich, cat. no. 85402)

- Sigmacote (Sigma-Aldrich, cat. no. SL2)

- Mercury (Sigma-Aldrich, cat. no. 261017)

- NP-40 Surfact-Amps Detergent Solution (Thermo Fisher Scientific, cat. no. 28324)

- Dimethylpolysiloxane (20 cSt; Sigma-Aldrich, cat. no. DMPS2X, or 50 cSt; Sigma-Aldrich, cat. no. DMPS5X)

Trim-Away by electroporation in cell lines and primary cells

- DMEM (high-glucose; GlutaMAX supplement; pyruvate; Gibco, cat. no. 31966)

- FBS (Gibco, cat. no. 10270)

- DPBS (no calcium; no magnesium; Gibco, cat. no. 14190)

- Trypsin-EDTA (0.05\% (wt/vol), phenol red; Gibco, cat. no. 25300) 
- Mouse anti-TRIM21 (D-12; Santa Cruz Biotechnology, cat. no. sc-25351)

- Rabbit anti-TRIM21 (D1O1D; Cell Signaling Technology, cat. no. 92043S)

\section{Equipment}

Antibody preparation

- Amicon Ultra-0.5 centrifugal filter unit with Ultracel-100 membrane (Merck Millipore, cat. no. UFC100)

- Vivaspin 500 (100,000 MWCO PES; Sartorius, cat. no. VS0141)

Isolation and culture of mouse oocytes and pre-implantation embryos

- TC-treated easy-grip-style cell culture dish (35 mm; Corning, cat. no. 353001)

- Microcapillary tube (100 $\mu$ l; Sigma-Aldrich, cat. no. P1174)

- Aspirator tube assemblies for calibrated microcapillary pipettes (Sigma-Aldrich, cat. no. A5177)

- Stereomicroscope (Zeiss, cat. no. SteREO Discovery.V8)

- Galaxy 48R (Eppendorf, cat. no. CO482)

Constitutive Trim-Away in mouse oocytes and pre-implantation embryos

- Inverted microscope (Zeiss, cat. no. Axio Vert.A1)

- Crossline micrometer (Zeiss, cat. no. 474066-9901-000)

- Vibration-free table (Gutmann, cat. no. 14.860)

- Stage micrometer for transmitted light (Zeiss, cat. no. 474026-0000-000)

- Coarse manipulator (Narishige, cat. no. MN-4)

- Three-axis joystick oil hydraulic micromanipulator (Narishige, cat. no. MO-202U)

- Zeiss 2 adapter (for Eppendorf micromanipulation systems; Eppendorf, cat. no. 5192312002)

- TransferMan 4r (Eppendorf, cat. no. 5193000012)

- $22 \times 22$ mm no. 0 glass coverslips (Sigma-Aldrich, cat. no. C9802)

- Diamond pencil (CellPath, cat. no. MEA-0301-00A)

- Microinjection chamber (design adapted from ref. ${ }^{31}$ )

- Capillary that fits into the groove of the microinjection chamber (e.g., Drummond Scientific, cat. no. 1-00-0300)

- Microcaps (Drummond Scientific, cat. no. 1-000-0500)

- Flaming/Brown micropipette puller (Sutter Instrument, cat. no. P-97)

- Calibrated syringe (10 $\mu \mathrm{l}$; Hamilton, cat. no. 80300)

- CellTram Oil (Eppendorf, cat. no. 5176000076)

- Microloader (Eppendorf, cat. no. 5242956003)

- Femtotip II (Eppendorf, cat. no. 5242957000)

- FemtoJet 4i (Eppendorf, cat. no. 5252000013)

- Holding capillary (VacuTip: Eppendorf, cat. no. 5175 108.000, or VacuTip FCH: Eppendorf, cat. no. 5175 240.006)

- CellTram Air (Eppendorf, cat. no. 5176000068)

- TC-treated easy-grip-style cell culture dish (60 mm; Corning, cat. no. 353004)

Trim-Away by electroporation in cell lines and primary cells

- Corning tissue culture-treated culture dishes (100 mm $\times 20 \mathrm{~mm}$; Sigma-Aldrich, cat. no. CLS430167)

- Tube (15 ml; polypropylene; screw cap; Greiner Bio-One, cat. no. 188285)

- Eppendorf Safe-Lock tubes (1.5 ml; Eppendorf, cat. no. 0030120086)

- Neon Transfection System starter pack (Invitrogen, cat. no. MPK5000S)

\section{Reagent setup}

Trim21 and reporter mRNA

mRNAs encoding Trim21 and reporters for live-cell imaging such as meGFP-MAP4 and $\mathrm{H} 2 \mathrm{~B}-\mathrm{mCherry}$ are prepared by in vitro transcription as described in Box 4 and can be stored at $-80{ }^{\circ} \mathrm{C}$ as long as no signs of mRNA degradation can be seen.

TRIM21-overexpressing stable cell lines

TRIM21-overexpressing cell lines are prepared as described in Box 1 and can be cultivated at $37^{\circ} \mathrm{C} / 5 \% \mathrm{CO}_{2}$ as long as no signs of senescence can be seen. 


\section{Box 4 | Trim21 and reporter mRNA preparation Timing $8 \mathrm{~h}$}

This box describes the procedures for preparing mRNAs encoding Trim21 and reporters for live-cell imaging. The resulting mRNAs are suitable for microinjection into mouse oocytes or early embryos for expression.

\section{Reagents}

- pGEMHE-mCherry-mTrim21 (Addgene, plasmid no. 105522)

- pGEMHE-mEGFP-mTrim21 RRING-Box (Addgene, plasmid no. 105520)

- pGEMHE-mCherry-mTrim21 RRING-Box (Addgene, plasmid no. 105523)

- pGEMHE-mEGFP-mTrim21 2 PRYSPRY (Addgene, plasmid no. 105521)

- pGEMHE-mCherry-mTrim21 2 PRYSPRY (Addgene, plasmid no. 105524)

- Nuclease-free water (not DEPC-treated; Invitrogen, cat. no. AM9930)

- CutSmart buffer (NEB, cat. no. B7204S)

- Ascl (NEB, cat. no. R0558S or R0558 L)

- Glycogen (RNA grade; Thermo Fisher Scientific, cat. no. R0551)

- Phenol-chloroform-isoamyl alcohol (IAA) mixture (25:24:1; Sigma-Aldrich, cat. no. 77617)

- Sodium acetate buffer solution (3 M; pH 7.0; Sigma-Aldrich, cat. no. S2404)

- Ethanol (Sigma-Aldrich, cat. no. 51976)

- Liquid nitrogen (Sigma-Aldrich, cat. no. 00474-1L)

- HiScribe T7 ARCA mRNA Kit (with tailing; NEB, cat. no. E2060S)

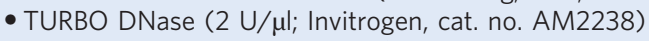

- NucleoSpin RNA Clean-up XS (Macherey-Nagel, cat. no. 740903)

- Phenol-chloroform-IAA mixture (125:24:1; Sigma-Aldrich, cat. no. 77619)

- Ethylenediaminetetraacetic acid solution (0.5 M; pH 8.0; Sigma-Aldrich, cat. no. 03690)

- Sodium acetate buffer solution (3 M; pH 5.2; Sigma-Aldrich, cat. no. S7899)

- Sodium dodecyl sulfate (SDS) solution (10\% (wt/vol) in $\mathrm{H}_{2} \mathrm{O}$; Sigma-Aldrich, cat. no. 71736)

\section{Equipment}

- Snaplock microcentrifuge tube (1.5 ml; RNase/DNase-free; nonpyrogenic; Corning, cat. no. MCT-150-C)

- Centrifuge, model no. 5424R (refrigerated; Eppendorf, cat. no. 5404000413)

- NanoDrop 2000 spectrophotometer (Thermo Fisher Scientific, cat. no. ND-2000)

\section{Reagent setup}

- $0.25 \mathrm{M}$ sodium acetate-5 mM EDTA-0.5\% (wt/vol) SDS. Add $0.1 \mathrm{ml}$ of $0.5 \mathrm{M}$ EDTA solution, $0.5 \mathrm{ml}$ of $10 \%$ (wt/vol) SDS solution and $0.833 \mathrm{ml}$ of $3 \mathrm{M}$ sodium acetate buffer solution (pH 5.2) to $8.567 \mathrm{ml}$ of nuclease-free water. Divide into $1-\mathrm{ml}$ aliquots and store at $-20{ }^{\circ} \mathrm{C}$ (for up to 1 year) until use.

- $70 \%$ ( vol/ $\mathrm{vol}$ ) ethanol. Add $35 \mathrm{ml}$ of ethanol to $15 \mathrm{ml}$ of nuclease-free water. Store at $-20^{\circ} \mathrm{C}$ (for up to 1 year) until use.

\section{Equipment setup}

- NanoDrop 2000 spectrophotometer. Set up the NanoDrop 2000 spectrophotometer according to the manufacturer's instructions.

\section{Procedure}

1 Linearization and purification of DNA template (steps 1-12). Mix the components listed below in a 1.5-ml nuclease-free tube and incubate at $37^{\circ} \mathrm{C}$ for $1 \mathrm{~h}$.

\section{Component}

Nuclease-free water

$0.5 \mu \mathrm{g} / \mu \mathrm{l}$ Trim21 or reporter construct (in pGEMHE)

$10 \times$ CutSmart buffer

$10 \mathrm{U} / \mu \mathrm{l} \mathrm{Ascl}$

\section{Amount per reaction $(\mu \mathrm{l}) \quad$ Final concentration}

20

20

5

5

$\triangle$ CRITICAL STEP We generally use pGEMHE as the vector because it contains a T7 promoter, multiple cloning sites flanked by 5'- and 3'-untranslated regions (UTRs) of the Xenopus $\beta$-globin gene, a short stretch of ( $35-\mathrm{bp})$ poly (A) sequence and linearization sites. Constructs in other vectors such as pCS2 can also be used, but the translation efficiency may be lower because of the lack of $5^{\prime}$ and $3^{\prime}$ UTRs, and poly(A) tailing may be required.

2 Add $0.5 \mu \mathrm{l}$ of glycogen, $150 \mu \mathrm{l}$ of nuclease-free water and $200 \mu \mathrm{l}$ of phenol-chloroform-IAA (pH 8.0) to terminate the linearization reaction.

$\triangle$ CRITICAL STEP Addition of glycogen as a co-precipitant is optional, but the yield of precipitated DNA is higher.

$\triangle$ CRITICAL STEP Phenol-chloroform-IAA is saturated and stored with an equilibration buffer. Do not take in the upper aqueous layer to avoid dilution.

3 Invert end-to-end to mix well and centrifuge at $20,000 \mathrm{~g}$ for $5 \mathrm{~min}$ at room temperature.

4 Transfer $\sim 180 \mu \mathrm{l}$ of the upper phase to a new $1.5-\mathrm{ml}$ nuclease-free tube, add $20 \mu \mathrm{l}$ of $3 \mathrm{M}$ sodium acetate $(\mathrm{pH} 7.0)$ and $400 \mu \mathrm{l}$ of ice-cold $100 \%$ ( $\mathrm{vol} / \mathrm{vol})$ ethanol.

$\triangle$ CRITICAL STEP Do not use isopropanol, as more salts will be precipitated.

5 Invert end-to-end to mix well and snap-freeze in liquid nitrogen. 


\section{Box 4 | (continued)}

6 Centrifuge at $20,000 \mathrm{~g}$ for $30 \mathrm{~min}$ at $4^{\circ} \mathrm{C}$.

$\triangle$ CRITICAL STEP Start centrifugation before the mixture thaws.

7 Discard the supernatant and add $1 \mathrm{ml}$ of ice-cold $70 \%$ ( $\mathrm{vol} / \mathrm{vol}$ ) ethanol in nuclease-free water.

8 Invert end-to-end to mix well and centrifuge at $20,000 \mathrm{~g}$ for $1 \mathrm{~min}$ at $4{ }^{\circ} \mathrm{C}$.

$\triangle$ CRITICAL STEP Do not vortex, in order to avoid fragmentation of the pellet.

9 Discard $~ 900 \mu \mathrm{l}$ of the supernatant and centrifuge at $20,000 \mathrm{~g}$ for $30 \mathrm{~s}$ at $4{ }^{\circ} \mathrm{C}$.

$\triangle$ CRITICAL STEP This step is necessary for avoiding ethanol carryover.

10 Discard the rest of the supernatant and air-dry for $3 \mathrm{~min}$ at room temperature.

$\triangle$ CRITICAL STEP After drying, the center of the pellet should remain white, with the edge being transparent. Over-drying, as indicated by a completely transparent pellet, should be avoided.

11 Add $6 \mu \mathrm{l}$ of nuclease-free water and pipette up and down to fully dissolve the pellet.

12 Use $1 \mu \mathrm{l}$ to measure the DNA concentration with a NanoDrop spectrophotometer. We typically recover $\sim 7-8 \mu \mathrm{g}$ of linearized DNA.

PAUSE POINT Linearized DNA can be stored at $-20{ }^{\circ} \mathrm{C}$ for up to 1 week until use.

13 In vitro transcription of capped mRNA (step 13). Mix the components listed below in a 1.5-ml nuclease-free tube and incubate at $40{ }^{\circ} \mathrm{C}$ for $4 \mathrm{~h}$.

\section{Component}

Amount per reaction $(\mu l)$

Nuclease-free water 19 or 21.5

$\sim 1.2 \mu \mathrm{g} / \mu \mathrm{l}$ linearized DNA

2x ARCA/NTP mix

2.5 (for mRNA of $<6 \mathrm{~kb}$ ) or 5 (for mRNA of $\geq 6 \mathrm{~kb}$ )

30

T7 RNA polymerase mix 6

Final concentration

$\triangle$ CRITICAL STEP We use the HiScribe T7 ARCA mRNA Kit (with tailing) for successful transcription of fulllength mRNAs of up to $12 \mathrm{~kb}$ at high yield. Other in vitro transcription kits can also be used, but the ratio of ARCA:GTP:CTP:ATP:UTP should be adjusted from 0.8:0.2:1:1:1 to 4:1:1:1:1.

$\triangle$ CRITICAL STEP For constructs with other promoters such as T3 or SP6, the corresponding RNA polymerase should be used.

14 Removal of DNA template (step 14). Add $3 \mu$ of $2 \mathrm{U} / \mu \mathrm{l}$ TURBO DNase and incubate at $37{ }^{\circ} \mathrm{C}$ for $15 \mathrm{~min}$. $\triangle$ CRITICAL STEP Other DNase I can be used as well.

15 (Optional) Poly(A) tailing of mRNA (steps 15 and 16). Mix the components listed below and incubate at $37^{\circ} \mathrm{C}$ for 3 min.

\section{Component}

Amount per reaction $(\mu \mathrm{l})$

Final concentration

Nuclease-free water

192

Mix from step 13

$10 \times \operatorname{Poly}(A)$ polymerase reaction buffer

$1 \times$

$\triangle$ CRITICAL STEP We generally omit steps 15 and 16 when we synthesize mRNAs from pGEMHE-based plasmids, and instead add $237 \mu \mathrm{l}$ of nuclease-free water and directly proceed to step 17 . However, poly(A) tailing can be advantageous for assays with a longer time frame (i.e., $>24 \mathrm{~h}$ ) or for mRNAs transcribed from constructs without a short stretch of poly $(A)$ tail.

16 Add $15 \mu \mathrm{l}$ of poly(A) polymerase and incubate at $37^{\circ} \mathrm{C}$ for $30 \mathrm{~min}$.

17 Removal of free nucleotides (step 17). Purify the mRNA using NucleoSpin RNA Clean-up XS and elute with $160 \mu$ l of nuclease-free water.

$\triangle$ CRITICAL STEP This step can be omitted, but then the final RNA concentration cannot be directly determined with a NanoDrop spectrophotometer because of free-nucleotide contamination.

18 Purification of mRNA (steps 18-23). Add $0.5 \mu$ l of glycogen, $40 \mu$ l of $0.25 \mathrm{M}$ sodium acetate- $5 \mathrm{mM}$ EDTA-0.5\% (wt/vol) SDS and $200 \mu \mathrm{l}$ of phenol-chloroform-IAA (pH 4.0).

$\triangle$ CRITICAL STEP Addition of glycogen as a co-precipitant is optional, but the yield of precipitated mRNA is higher.

$\triangle$ CRITICAL STEP Phenol-chloroform-IAA is saturated and stored with an equilibration buffer. Do not take in the upper aqueous layer to avoid dilution.

19 Invert end-to-end to mix well and centrifuge at $20,000 \mathrm{~g}$ for $5 \mathrm{~min}$ at room temperature.

20 Transfer $\sim 180 \mu \mathrm{l}$ of the upper phase to a new $1.5-\mathrm{ml}$ nuclease-free tube and add $360 \mu \mathrm{l}$ of ice-cold $100 \%$ (vol/vol) ethanol.

$\triangle$ CRITICAL STEP Do not use $100 \%$ isopropanol, as more salts will be precipitated.

21 Repeat steps 5-10.

22 Add $11 \mu \mathrm{l}$ of nuclease-free water and pipette up and down to fully dissolve the pellet.

23 Use $1 \mu$ l to measure the RNA concentration with a NanoDrop spectrophotometer.

$\triangle$ CRITICAL STEP RNA concentration can also be determined with other techniques such as agarose gel electrophoresis and comparison with RNA markers of known concentration or fluorescence-based Qubit RNA assays.

$\triangle$ CRITICAL STEP We typically obtain mRNA at $\sim 1-3 \mu \mathrm{g} / \mu \mathrm{l}$.

PAUSE POINT mRNA should be divided into $0.6-\mu \mathrm{l}$ single-use aliquots and can be stored at $-80^{\circ} \mathrm{C}$ as long as no signs of mRNA degradation can be seen. 
Recombinant TRIM21 protein

TRIM21 protein is prepared by purification from bacteria as described in Box 2 and can be stored at $-80{ }^{\circ} \mathrm{C}$ as long as no signs of protein degradation can be seen.

\section{PBS for antibody preparation}

Dissolve $5 \mathrm{~g}$ of PBS tablets in $500 \mathrm{ml}$ of embryo-tested or nuclease-free water. Store at room temperature as long as no signs of contamination can be seen.

Homemade culture medium for isolating mouse oocytes and pre-implantation embryos We routinely use modified M2 medium as described in ref. ${ }^{34}$ for culture of mouse oocytes and preimplantation embryos. The modified M2 medium contains $5.53 \mathrm{~g} /$ liter $\mathrm{NaCl}, 0.36 \mathrm{~g} /$ liter $\mathrm{KCl}$, $0.25 \mathrm{~g} /$ liter $\mathrm{CaCl}_{2} \bullet 2 \mathrm{H}_{2} \mathrm{O}, 0.16 \mathrm{~g} /$ liter $\mathrm{KH}_{2} \mathrm{PO}_{4}, 0.29 \mathrm{~g} /$ liter $\mathrm{MgSO}_{4} \bullet 7 \mathrm{H}_{2} \mathrm{O}, 0.35 \mathrm{~g} /$ liter $\mathrm{NaHCO}_{3}, 4.97$ g/liter HEPES, $2.6 \mathrm{~g} /$ liter Na lactate, $0.04 \mathrm{~g} /$ liter Na pyruvate, $1.1 \mathrm{~g} /$ liter glucose monohydrate, $4 \mathrm{~g} /$ liter BSA, $0.06 \mathrm{~g} /$ liter penicillin $\mathrm{G}, 0.05 \mathrm{~g} / \mathrm{liter}$ streptomycin and $0.002 \mathrm{~g} /$ liter phenol red. We reduce the concentration of phenol red from $0.01 \mathrm{~g} /$ liter to $0.002 \mathrm{~g} / \mathrm{liter}$, as phenol red is toxic for mouse oocytes and early embryos. Store at $4{ }^{\circ} \mathrm{C}$ for up to 2 weeks. $\triangle$ CRITICAL We obtain higher developmental rates with homemade M2 medium than with commercial M2 medium. $\triangle$ CRITICAL Drugs such as $250 \mu \mathrm{M}$ dibutyryl cyclic adenosine monophosphate should be added to the culture medium to maintain meiotic arrest for GV oocytes.

\section{DMEM supplemented with $1 \times$ GlutaMAX and $10 \%$ (vol/vol) FBS}

Add $55 \mathrm{ml}$ of FBS to $500 \mathrm{ml}$ of DMEM. Store at $4{ }^{\circ} \mathrm{C}$ for up to 1 year from the date of manufacture until use.

\section{Equipment setup}

Mouth pipette for isolation and culture of mouse oocytes and pre-implantation embryos Pull microcapillary tubes above a gas burner. Break the tip by striking a pair of pipettes against each other to obtain a smooth opening of $\sim 80-100 \mu \mathrm{m}$. Attach a pipette to the aspirator tube assembly. More details on mouth pipettes can be found in ref. ${ }^{35}$.

\section{Microinjection setup}

Install the inverted microscope on a vibration-free table. For the use of hydraulics-based systems, mount the coarse manipulator and the three-axis joystick oil hydraulic micromanipulator on one side of the arm. For the use of pressure-based systems, install the Adapter Zeiss 2 and mount a TransferMan $4 \mathrm{r}$ on both sides of the arm.

\section{Coverslips}

Boil 0.5 liter of deionized water in a microwave oven, add a few drops of dishwashing detergent and add $22 \times 22 \mathrm{~mm}$ no. 0 glass coverslips one by one. Incubate for $20 \mathrm{~min}$ at room temperature and discard the detergent solution. Rinse with deionized water three times, boil and rinse with deionized water three times again. Transfer the coverslips one by one to 0.5 liter of $5 \mathrm{M}$ hydrochloric acid and incubate overnight at room temperature. Rinse five times with deionized water and three times with double-distilled water. Transfer the coverslips one by one to $70 \%$ (vol/vol) ethanol for storage. Blotdry excess ethanol solution and air-dry before use. $\triangle$ CRITICAL Using unwashed coverslips for microinjection may compromise the survival rate of oocytes and early embryos during microinjection.

\section{Microinjection chamber}

Cut a washed coverslip along the edges into six equal rectangular pieces (shelves) and a center square piece with a diamond pencil. Attach a piece of double-sided tape of approximately the same dimensions as a shelf (as a spacer of $\sim 100 \mu \mathrm{m}$ ) close to the center of another washed coverslip at $\sim 5 \mathrm{~mm}$ from one edge. Attach a shelf to the double-sided tape to form an $\sim 0.5$ - $\mathrm{mm}$ loading space at the side closest to the edge. Apply a thin layer of silicon grease along the U-shaped cut on both sides of the microinjection chamber. Attach the coverslip, with the loading space facing away from the U-shaped cut, to the side of the microinjection chamber with a groove for placing the capillary. Attach another washed coverslip to the other side of the microinjection chamber. Gently press on the coverslips to spread the silicon grease. Fill the U-shaped reservoir with culture medium, and keep the microinjection chamber in a humidified container at $37{ }^{\circ} \mathrm{C}$ before use. After loading and before 
microinjection, apply a thin layer of silicon grease along the groove and insert the capillary with mRNA and/or antibody.

\section{Microinjection needle}

Siliconize the microcaps by dipping one end into a beaker containing $\sim 0.5 \mathrm{~cm}$ of Sigmacote. A column of Sigmacote will form as a result of capillary action. Invert the microcaps end to end several times so that the entire length is fully covered with Sigmacote. Blot-dry excess Sigmacote at one end and air-dry for at least 2 weeks. Pull siliconized microcaps in a Flaming/Brown micropipette puller to obtain pairs of pipettes with an opening of $\sim 0.5-1 \mu \mathrm{m}$ after breaking the tip. Back-load $\sim 1 \mu \mathrm{l}$ of mercury as close to the tip as possible with a $10-\mu$ l calibrated syringe. $\triangle$ CRITICAL Siliconizing the microcaps largely facilitates the flow of mercury within the microinjection needle. $\triangle$ CRITICAL Insufficient drying of Sigmacote increases the chance of breaking the mercury column during microinjection.

\section{CellTram oil}

Set up the CellTram Oil according to the manufacturer's instructions. Fill it with mineral oil.

\section{FemtoJet $4 \mathbf{i}$}

Set up the FemtoJet $4 \mathrm{i}$ according to the manufacturer's instructions.

\section{CellTram Air}

Set up the CellTram Air according to the manufacturer's instructions.

Neon Transfection System for electroporation in primary cells and cell lines

The Neon Transfection System should be set up according to the manufacturer's instructions.

\section{Procedure}

\section{Antibody preparation for Trim-Away Timing 1-2 h}

$\triangle$ CRITICAL This section describes how to prepare the antibody of interest for Trim-Away. Ultrafiltration is necessary for removing contaminants that are cytotoxic (e.g., sodium azide) or interfere with microinjection (e.g., glycerol).

1 If the antibody is from an unpurified source such as serum, ascites fluid or cell culture supernatant, or contains BSA or gelatin, pre-purify the antibody using the $0.2-\mathrm{ml}$ NAb protein A/G Spin Kit.

$\triangle$ CRITICAL STEP This step is necessary for removing contaminating proteins, which lead to an overestimation of the IgG concentration. Gelatin is not compatible with the Amicon Ultra- 0.5 $100-\mathrm{K}$ devices used in Step 3.

2 Mix the components listed below in a $1.5-\mathrm{ml}$ nuclease-free tube on ice.

\begin{tabular}{lll} 
Component & Amount per reaction $(\mu \mathrm{l})$ & Final concentration \\
\hline PBS (in embryo-tested or nuclease-free water) & $400-450$ & \\
$1 \mathrm{mg} / \mathrm{ml}$ antibody & $50-100$ & $0.1-0.2 \mu \mathrm{g} / \mu \mathrm{l}$
\end{tabular}

$\triangle$ CRITICAL STEP For co-injection of Trim21 mRNA and antibody using FemtoJet, PBS should be prepared in nuclease-free water instead to reduce the chance of RNase contamination.

3 Insert an Amicon Ultra-0.5 100-K device into a provided collection tube.

4 Apply $500 \mu \mathrm{l}$ of diluted antibody from Step 2 to the device.

5 Align the cap strap of the collection tube and the membrane of the device toward the center of the rotor.

6 Centrifuge at $14,000 \mathrm{~g}$ for $10 \mathrm{~min}$ at $4{ }^{\circ} \mathrm{C}$.

$\triangle$ CRITICAL STEP Higher centrifugal force may damage the membrane and cause sample loss.

7 Discard the flow-through.

8 Add $480 \mu \mathrm{l}$ of PBS to the Amicon Ultra-0.5 100-K device and carefully mix well.

$\triangle$ CRITICAL STEP Mixing is necessary to avoid a high local concentration during ultrafiltration, which can cause protein aggregation and loss. Avoid touching the membrane with the pipette tip, as this may damage the membrane and cause sample loss. 
9 Repeat Steps 5-7 twice.

\section{? TROUBLESHOOTING}

10 Insert the inverted Amicon Ultra-0.5 100-K device into another provided collection tube.

11 Align the open cap toward the center of the rotor.

12 Centrifuge at $1,000 \mathrm{~g}$ for $2 \mathrm{~min}$ at $4{ }^{\circ} \mathrm{C}$.

13 Use $2 \mu \mathrm{l}$ to measure the IgG concentration with a NanoDrop spectrophotometer.

$\triangle$ CRITICAL STEP $1.0 \mathrm{OD}_{280}$ is equivalent to $0.714 \mathrm{mg} / \mathrm{ml} \mathrm{IgG}$.

$\triangle$ CRITICAL STEP We typically retrieve $\sim 20 \mu \mathrm{l}$ of purified antibody at $\sim 1.25-2.5 \mathrm{mg} / \mathrm{ml}$ from $50 \mu \mathrm{g}$ of input.

? TROUBLESHOOTING

14 Snap-freeze in liquid nitrogen.

$\triangle$ CRITICAL STEP The purified antibody should be divided into 1 - to 2 - $\mu$ l single-use aliquots to minimize freeze-thaw cycles in the absence of cryoprotectant.

PAUSE POINT The antibody can be stored at $-80^{\circ} \mathrm{C}$ as long as no loss of activity can be seen.

Isolation and culture of mouse oocytes and pre-implantation embryos $\bigcirc$ Timing $>\mathbf{1 ~ h}$

15 Isolate and culture mouse GV, meiosis II (MII) oocytes or early embryos in oil as previously described in refs. ${ }^{36-41}$.

Trim-Away by microinjection of mouse oocytes and pre-implantation embryos Timing 4-5 h

16 To perform constitutive depletion (Fig. 3a) using hydraulic systems, follow option A. To perform constitutive depletion using pressure-based systems, follow option B. To perform acute depletion (Fig. 3b), follow option C.

(A) Constitutive depletion using hydraulic-based systems such as CellTram Oil $\bigcirc$ Timing 4-5 h

(i) Mix the components listed below in a 1.5-ml nuclease-free tube on ice.

\begin{tabular}{lll} 
Component & Amount per reaction $(\mu \mathrm{l})$ & Final concentration \\
\hline $2 \mu \mathrm{g} / \mu \mathrm{l}$ Trim21 mRNA & 0.5 & $400 \mathrm{ng} / \mu \mathrm{l}$ \\
Reporter mRNA(s) & Variable & Usually $30-300 \mathrm{ng} / \mu \mathrm{l}$ each \\
Nuclease-free water & To 2.5 &
\end{tabular}

PAUSE POINT The mRNA mix can be stored at $-20^{\circ} \mathrm{C}$ or $-80^{\circ} \mathrm{C}$ (for up to 1 year) until use. We usually freeze-thaw the mRNA mix up to four times without noticeable RNA degradation.

(ii) Mix the components listed below in another 1.5-ml nuclease-free tube on ice.

\begin{tabular}{lll} 
Component & $\begin{array}{l}\text { Amount per } \\
\text { reaction }(\mu \mathrm{l})\end{array}$ & Final concentration \\
\hline $2 \mathrm{mg} / \mathrm{ml}$ antibody/antibodies from Step 14 & 1.25 & $1 \mathrm{mg} / \mathrm{ml}$ each \\
$0.5 \%$ (vol/vol) NP-40 (in embryo-tested water) & $0.25-0.5$ & $0.05-0.1 \%$ (vol/vol) \\
PBS (in embryo-tested water) & To 2.5 & \\
\hline
\end{tabular}

$\triangle$ CRITICAL STEP Antibody at a final concentration of $1 \mathrm{mg} / \mathrm{ml}$ is a good starting point for complete depletion of most target proteins. However, we suggest titrating individual antibodies to the minimal effective concentration, as some can have secondary inhibitory effects.

$\triangle$ CRITICAL STEP For antibody at a final concentration of $<0.1 \mathrm{mg} / \mathrm{ml}$, storage in a lowprotein-binding tube or preparation of fresh antibody mix is recommended.

$\triangle$ CRITICAL STEP NP-40 largely facilitates the flow of the viscous antibody mix within the microinjection needle and reduces the chance of clogging.

DAUSE POINT For short-term storage (up to 1 week), the antibody mix can be stored at $4{ }^{\circ} \mathrm{C}$ in a $1.5-\mathrm{ml}$ tube with the opening sealed with Parafilm. However, changes in ionic strength due to repeated evaporation and condensation at the cap can denature the antibody/antibodies. For long-term storage (up to at least 1 month), the antibody mix can 
be stored at $-20{ }^{\circ} \mathrm{C}$ or $-80{ }^{\circ} \mathrm{C}$ until use. We usually freeze-thaw the antibody mix up to four times without noticeable loss of activity.

(iii) Centrifuge the mRNA mix and antibody mix at 20,000 $\mathrm{g}$ for $5 \mathrm{~min}$ at $4{ }^{\circ} \mathrm{C}$ before capillary loading.

$\triangle$ CRITICAL STEP This step is necessary for reducing the amount of precipitated material loaded into the capillary.

(iv) Load $0.5 \mu \mathrm{l}$ of dimethylpolysiloxane into a capillary.

$\triangle$ CRITICAL STEP Avoid introducing air bubbles from Step 16A(iv) to Step 16A(viii).

$\triangle$ CRITICAL STEP Follow the order of loading (Fig. 3c) from Step 16A(iv) to Step 16A(viii). ? TROUBLESHOOTING

(v) Load $0.5 \mu \mathrm{l}$ of precleared antibody mix.

$\triangle$ CRITICAL STEP As little as $0.25 \mu \mathrm{l}$ can be used.

? TROUBLESHOOTING

(vi) Load $0.5 \mu \mathrm{l}$ of dimethylpolysiloxane.

? TROUBLESHOOTING

(vii) Load $0.5 \mu \mathrm{l}$ of precleared mRNA mix.

$\triangle$ CRITICAL STEP As little as $0.25 \mu \mathrm{l}$ can be used. ? TROUBLESHOOTING

(viii) Load $0.5 \mu \mathrm{l}$ of dimethylpolysiloxane.

$\triangle$ CRITICAL STEP The loaded capillary should be kept on ice until use. ? TROUBLESHOOTING

(ix) Transfer oocytes or early embryos to the loading space of the microinjection chamber using a mouth pipette under the stereomicroscope.

(x) Mount a microinjection needle onto the capillary holder attached to the CellTram Oil.

(xi) Attach the capillary holder to the micromanipulator.

(xii) Carefully tap the tip of the microinjection needle against the wall of the capillary to obtain an opening of $\sim 0.5-1 \mu \mathrm{m}$ and slowly push the mercury column to the front.

$\triangle$ CRITICAL STEP An opening of $>1 \mu \mathrm{m}$ will increase the risk of lysis after microinjection, but an opening of $<0.5 \mu \mathrm{m}$ will increase the chance of clogging.

(xiii) Determine the injection volume by loading different amounts of polydimethylsiloxane into the microinjection needle and carefully expelling it into the mRNA mix. A polydimethylsiloxane droplet will form. The corresponding volume can be calculated using the formula $4 / 3 \times \pi \times(d / 2)^{3}$, in which $d$ is the diameter of the droplet.

(xiv) Load $\sim 1 \mathrm{pl}$ of polydimethylsiloxane into the microinjection needle.

(xv) Load $3.5 \mathrm{pl}$ of mRNA mix.

(xvi) Load $\sim 1 \mathrm{pl}$ of polydimethylsiloxane.

(xvii) Load $3.5 \mathrm{pl}$ of antibody mix.

(xviii) Load $\sim 1$ pl of polydimethylsiloxane.

$\triangle$ CRITICAL STEP The mRNA mix and antibody mix should not be mixed within the microinjection needle in order to avoid RNA degradation before microinjection.

$\triangle$ CRITICAL STEP The antibody mix can be injected at a volume of up to $8.5 \mathrm{pl}$ without harming the oocytes, which may be necessary for antibody/antibodies that cannot be concentrated to the desired concentration.

(xix) Adjust the level of the microinjection needle until the plasma membrane of the oocyte or early embryo is in focus.

(xx) Carefully press the tip of the microinjection needle against the oocyte or early embryo until it penetrates the plasma membrane.

(xxi) Expel the column of polydimethylsiloxane-mRNA mix-polydimethylsiloxane-antibody mix into the cytoplasm.

(xxii) Quickly withdraw the microinjection needle to release the oocyte or early embryo.

(xxiii) Repeat Steps 16A(xiv)-(xxii).

$\triangle$ CRITICAL STEP A successful microinjection is indicated by cytoplasmic movement and the presence of two dimethylpolysiloxane droplets.

$\triangle$ CRITICAL STEP A video and an illustration describing hydraulic-based microinjection step by step can be found at https://health.uconn.edu/cell-biology/wp-content/uploads/ sites/115/2017/07/follicleinjection.mp4?_=2 and in ref. ${ }^{41}$, respectively.

(xxiv) Transfer microinjected oocytes or early embryos from the microinjection chamber to a $50-\mu \mathrm{l}$ drop of culture medium covered with oil in a $35-\mathrm{mm}$ dish. 
(xxv) Incubate at $37^{\circ} \mathrm{C}$ for $3 \mathrm{~h}$ before performing an assay such as live imaging or immunofluorescence.

$\triangle$ CRITICAL STEP Full depletion of some target proteins can take up to $4 \mathrm{~h}$.

(B) Constitutive depletion using pressure-based systems such as the FemtoJet 4i $\bigcirc$ Timing 4-5 h

(i) Mix the components listed below in a $1.5-\mathrm{ml}$ nuclease-free tube on ice.

\begin{tabular}{lll} 
Component & $\begin{array}{l}\text { Amount per } \\
\text { reaction }(\mu \mathrm{l})\end{array}$ & Final concentration \\
\hline $2 \mu \mathrm{g} / \mu \mathrm{l}$ Trim21 mRNA & 0.25 & $200 \mathrm{ng} / \mu \mathrm{l}$ \\
Reporter mRNA(s) & Variable & Usually $15-150 \mathrm{ng} / \mu \mathrm{l}$ each \\
$2 \mathrm{mg} / \mathrm{ml}$ antibody/antibodies from Step 14 & 0.625 & $0.5 \mathrm{mg} / \mathrm{ml}$ each \\
$0.5 \%$ (vol/vol) NP-40 (in nuclease-free water) & $0.25-0.5$ & $0.05-0.1 \%(\mathrm{vol} / \mathrm{vol})$ \\
PBS (in nuclease-free water) & To 2.5 &
\end{tabular}

$\triangle$ CRITICAL STEP Antibody at a final concentration of $0.5 \mathrm{mg} / \mathrm{ml}$ is a good starting point for complete depletion of most target proteins. However, we suggest titrating individual antibodies to the minimal effective concentration, as some can have secondary inhibitory effects.

$\triangle$ CRITICAL STEP For antibody at a final concentration of $<0.1 \mathrm{mg} / \mathrm{ml}$, storage in a lowprotein-binding tube or preparation of fresh mRNA and antibody mix is recommended.

$\triangle$ CRITICAL STEP NP-40 largely facilitates the flow of viscous mRNA and antibody mix within the Femtotip II and reduces the chance of clogging.

PAUSE POINT The mRNA and antibody mix can be stored at $-20{ }^{\circ} \mathrm{C}$ or $-80{ }^{\circ} \mathrm{C}$ for up to 1 year until use. We usually freeze-thaw the mRNA and antibody mix up to four times without noticeable denaturation and/or RNA degradation.

(ii) Centrifuge the mRNA and antibody mix at 20,000 $\mathrm{g}$ for $5 \mathrm{~min}$ at $4{ }^{\circ} \mathrm{C}$ before loading.

$\triangle$ CRITICAL STEP This step is necessary for reducing the amount of precipitated materials loaded into the capillary.

(iii) Load $0.5 \mu \mathrm{l}$ of mRNA and antibody mix into the tip of a Femtotip II using a Microloader. $\triangle$ CRITICAL STEP Avoid introducing air bubbles.

(iv) Mount a Femtotip II onto the capillary holder of the FemtoJet 4i.

(v) Mount a holding capillary onto the capillary holder of the CellTram Air.

$\triangle$ CRITICAL STEP We have also successfully used the FemtoJet 4i with a microinjection chamber instead of a holding capillary.

(vi) Attach the capillary holders to the TransferMan 4r.

(vii) Transfer oocytes or early embryos to a drop of culture medium covered with oil on the lid of a $60-\mathrm{mm}$ dish.

(viii) Determine the appropriate settings for the microinjection program to inject a volume of $\sim 7 \mathrm{pl}$ by first microinjecting different amounts of a fluorescent dye into oocytes or early embryos and measuring the intracellular fluorescence intensity and the fluorescence intensity of a series of dye solutions of known concentration. The injection volume can then be calculated using the formula $F_{\text {dye injected }} \times V_{\text {dye injected }}=F_{\text {cell }} \times V_{\text {cell }}$, in which

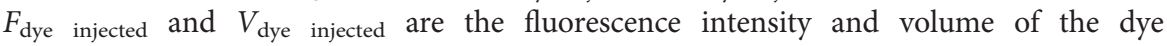
solution microinjected, respectively, and $F_{\text {cell }}$ and $V_{\text {cell }}$ are the fluorescence intensity and volume of the oocyte or early embryo microinjected, respectively. More details can be found in refs. ${ }^{42}$ and ${ }^{43}$.

$\triangle$ CRITICAL STEP This step depends on the experience of the scientist and may be optional.

$\triangle$ CRITICAL STEP The mRNA and antibody mix can be injected at a volume of up to $12 \mathrm{pl}$ without harming the oocytes, which may be necessary for antibody/antibodies that cannot be concentrated to the desired concentration.

(ix) Adjust the level of the holding capillary until the oocyte or early embryo is in focus. ? TROUBLESHOOTING

(x) Secure an oocyte or early embryo in place by applying a negative pressure at the holding capillary.

(xi) Adjust the level of the Femtotip II until the plasma membrane of the oocyte or early embryo is in focus.

? TROUBLESHOOTING 
(xii) Push the tip of the Femtotip II against the oocyte or early embryo until it penetrates the plasma membrane.

(xiii) Press the 'Inject' button on the FemtoJet $4 \mathrm{i}$ to expel $\sim 7 \mathrm{pl}$ of mRNA and antibody mix into the cytoplasm.

(xiv) Quickly withdraw the microinjection needle.

(xv) Release the oocyte or early embryo by applying a positive pressure at the holding capillary.

(xvi) Repeat Step 16B(ix-xv).

$\triangle$ CRITICAL STEP A successful microinjection is indicated by a cytoplasmic movement.

$\triangle$ CRITICAL STEP A video describing pressure-based microinjection step by step can be found in ref. ${ }^{44}$. ? TROUBLESHOOTING

(xvii) Transfer microinjected oocytes or early embryos from the stage to a 50- $\mu$ d drop of culture medium covered with oil in a $35-\mathrm{mm}$ dish.

(xviii) Incubate at $37{ }^{\circ} \mathrm{C}$ for $3 \mathrm{~h}$ before performing an assay such as live imaging or immunofluorescence.

$\triangle$ CRITICAL STEP Full depletion of some target proteins can take up to $4 \mathrm{~h}$. ? TROUBLESHOOTING

(C) Acute depletion Timing $>\mathbf{4 h}$

(i) Prepare the mRNA mix as in Step $16 \mathrm{~A}(\mathrm{i})$.

(ii) Microinject $3.5 \mathrm{pl}$ using a hydraulic-based system as in Step $16 \mathrm{~A}$ (iii-xix) or a pressurebased system as in Step 16B(ii-ix).

? TROUBLESHOOTING

(iii) Transfer microinjected oocytes or early embryos to a drop of culture medium covered with oil.

(iv) Incubate at $37^{\circ} \mathrm{C}$ until the desired developmental stage.

(v) Prepare the antibody mix as in Step $16 \mathrm{~A}$ (ii).

(vi) Repeat Step 16C(ii).

? TROUBLESHOOTING

(vii) Immediately proceed to an assay such as live imaging or immunofluorescence ? TROUBLESHOOTING

\section{Trim-Away by electroporation in primary cells and cell lines Timing $<1 \mathrm{~h}$}

17 Trypsinize the cells using standard cell culture procedures and transfer them to a 15-ml tube.

18 Pellet the cells at $300 \mathrm{~g}$ for $5 \mathrm{~min}$ at room temperature and discard the supernatant.

19 Resuspend the cell pellet in $10 \mathrm{ml}$ of PBS and count the cells.

$\triangle$ CRITICAL STEP The cells should be thoroughly resuspended in PBS to avoid cell clumping, which can provide inaccurate results in cell counting.

20 Transfer $n \times\left(8 \times 10^{5}\right)$ cells to a new 15 - $\mathrm{ml}$ tube (where $n=$ number of electroporation reactions). $\triangle$ CRITICAL STEP We routinely use $8 \times 10^{5}$ cells per electroporation reaction with a $10-\mu$ l Neon tip. However, it is possible to use any number of cells between $1 \times 10^{5}$ and $1.5 \times 10^{6}$, depending on the type and size of cells, without any detrimental effects on antibody delivery or cell survival.

21 Pellet the cells at $300 \mathrm{~g}$ for $5 \mathrm{~min}$ at room temperature and discard all but $\sim 1 \mathrm{ml}$ of supernatant.

22 Using residual supernatant, transfer the cells to a new $1.5-\mathrm{ml}$ tube.

23 Pellet the cells at $300 \mathrm{~g}$ for $5 \mathrm{~min}$ at room temperature. In the meantime, proceed with Step 24 .

24 During Step 23, insert a Neon Tube into the Neon Pipette Station and fill the Neon Tube with $3 \mathrm{ml}$ of Neon Buffer E.

25 Carefully remove the supernatant.

$\triangle$ CRITICAL STEP The supernatant should be removed carefully, using a Gilson pipette, to avoid disturbing the cell pellet and subsequent cell loss.

26 Resuspend the cell pellet in $n \times 10 \mu$ of Neon Buffer $\mathrm{R}$ (where $n=$ number of electroporation reactions).

$\triangle$ CRITICAL STEP Once the cells are resuspended in Neon Buffer R, it is important to proceed with electroporation immediately. Prolonged incubation ( $>30 \mathrm{~min}$ ) in Neon Buffer R can reduce cell viability.

27 Add $10.5 \mu \mathrm{l}\left(\sim 8 \times 10^{5}\right)$ of cells per electroporation reaction to 1.5 -ml tubes.

$\triangle$ CRITICAL STEP It is important to avoid introducing air bubbles, which can cause arcing during electroporation. 
a

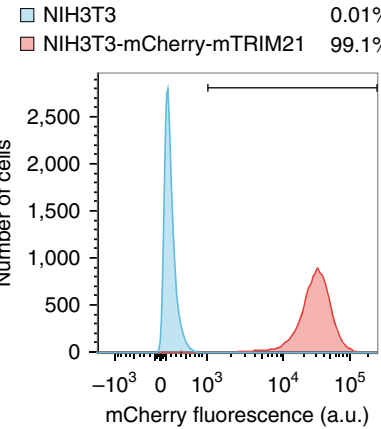

d

$\square$ HEK293T $\quad 0.04 \%$

$\square$ HEK293T-mCherry-hTRIM21 $99.6 \%$

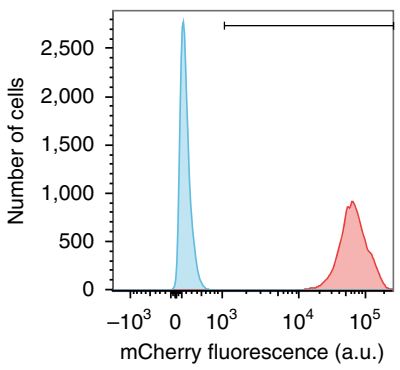

b

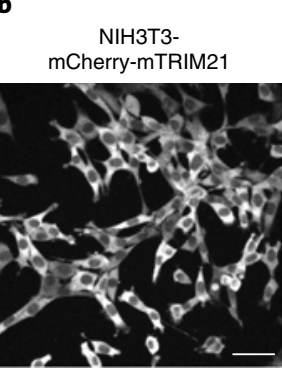

e

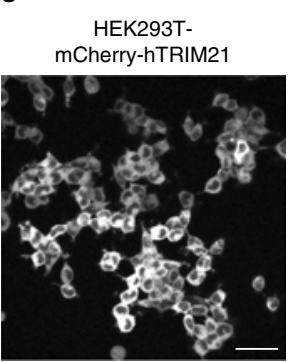

mCherry-TRIM21
Control IgG ++-
+-

Anti-Pcnt $-++\mathrm{kDa}$

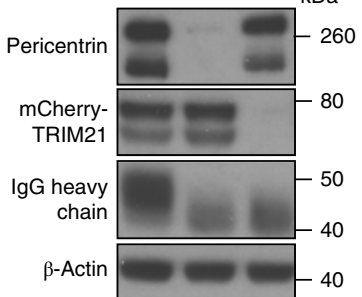

f

mCherry-TRIM21 +++

Anti-IKK $\alpha-++k \mathrm{kDa}$

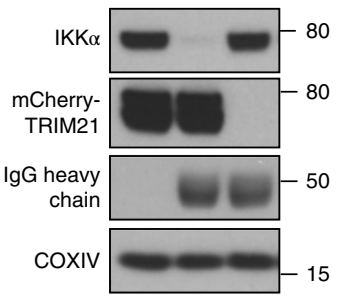

g

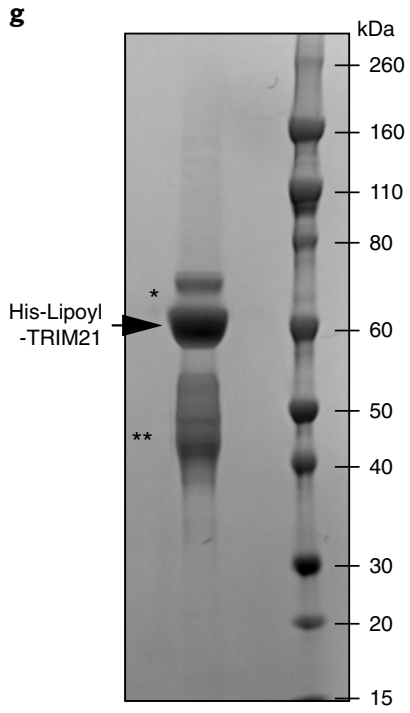

h
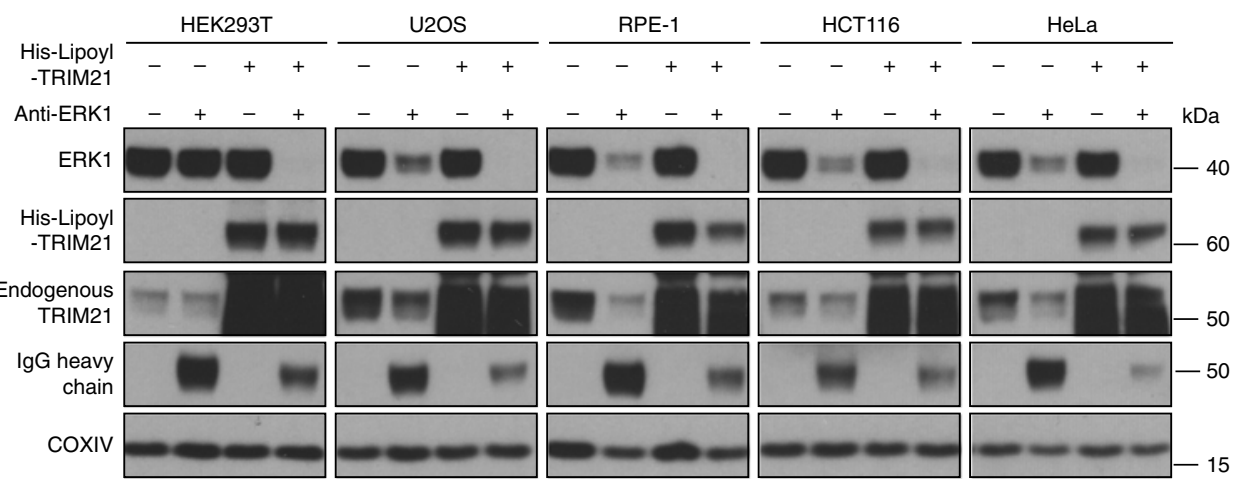

Fig. 2 | Trim-Away in cell lines. a,d, NIH3T3, NIH3T3-mCherry-mTRIM21, HEK293T and HEK293T-mCherry-hTRIM21 cell lines were analyzed by flow cytometry. At least 2,000 cells were counted for each condition. Percentages correspond to mCherry-positive cells falling within the gate drawn. $\mathbf{b}, \mathbf{e}$, NIH3T3-mCherry-mTRIM21 and HEK293T-mCherry-hTRIM21 cells were imaged by confocal microscopy, detecting mCherry fluorescence. Scale bars, $50 \mu \mathrm{m}$. c, NIH3T3 and NIH3T3-mCherry-mTRIM21 cell lines were electroporated with control lgG or anti-pericentrin antibodies and analyzed $16 \mathrm{~h}$ later by immunoblotting. f, HEK293T and HEK293T-mCherry-hTRIM21 cell lines were electroporated with PBS or anti-IKK $\alpha$ antibody and analyzed $3 \mathrm{~h}$ later by immunoblotting. $\mathbf{g}$, Coomassie-stained gel shows His-Lipoyl-hTRIM21 protein. Asterisks indicate bacterial contaminants $\left({ }^{*}\right)$ and degradation products $\left(^{\star \star}\right)$. h. The indicated cell lines were electroporated with PBS, anti-ERK1 antibody, His-Lipoyl-TRIM21 or anti-ERK1 + His-Lipoyl-TRIM21 and analyzed $3 \mathrm{~h}$ later by immunoblotting. a.u., arbitrary units; Pcnt, pericentrin. a-f and $\mathbf{h}$ adapted from ref. ${ }^{15}$ (https://doi.org/10.1016/j.cell.2017.10.033; original material licensed under a Creative Commons Attribution License 4.0).

28 Add $2 \mu$ of antibody from Step 14 to the cells and mix gently.

$\triangle$ CRITICAL STEP Antibody at a concentration of $1 \mathrm{mg} / \mathrm{ml}$ is a good starting point for complete depletion of most target proteins. However, we suggest titrating individual antibodies to the minimal effective concentration, as some can have secondary inhibitory effects.

$\triangle$ CRITICAL STEP It is important to avoid introducing air bubbles, which can cause arcing during electroporation. Gently tapping the tube can help to remove any air bubbles. A total volume of 
$12.5 \mu$ per electroporation reaction should be used, as this helps prevent any air bubbles being taken up into the 10- $\mu$ l Neon Tip in Step 28.

29 Take up the cell/antibody mix into the 10- $\mu$ Neon Tip.

$\triangle$ CRITICAL STEP It is essential to avoid taking up air bubbles into the $10-\mu l$ Neon Tip, as these can cause arcing during electroporation. Using a single smooth pipetting action combined with gentle swirling is recommended. Avoid pipetting up and down. For detailed instructions on how to insert the 10- $\mu$ l Neon Tip into the Neon Pipette, refer to the Neon Transfection System manual (https://tools.thermofisher.com/content/sfs/manuals/neon_device_man.pdf).

\section{? TROUBLESHOOTING}

30 Insert the Neon Pipette with the sample in the tip into the Neon Tube containing $3 \mathrm{ml}$ of Neon Buffer E assembled in the Neon Pipette Station. Push down vertically until a click is heard.

31 Electroporate using the following parameters: 1,400 V, two pulses, $20 \mathrm{~ms}$ per pulse.

$\triangle$ CRITICAL STEP If there is a visible spark, discard the sample and return to Step 28.

32 Transfer the sample to a $1.5-\mathrm{ml}$ tube containing $1 \mathrm{ml}$ of prewarmed cell growth medium without antibiotics. Electroporated cells can now be plated as determined by the scientist. An incubation time of $\sim 3 \mathrm{~h}$ is usually sufficient for target protein degradation.

? TROUBLESHOOTING

\section{Troubleshooting}

Troubleshooting advice can be found in Table 1.

Table 1 | Troubleshooting table

\begin{tabular}{|c|c|c|c|}
\hline Step & Problem & Possible reason & Solution \\
\hline \multicolumn{4}{|c|}{ Antibody preparation } \\
\hline \multirow[t]{3}{*}{9} & $\begin{array}{l}\text { The antibody cannot be concen- } \\
\text { trated further than to } \sim 20 \mu \mathrm{l}\end{array}$ & $\begin{array}{l}\text { The membrane is clogged with } \\
\text { precipitated materials }\end{array}$ & $\begin{array}{l}\text { Pre-clear the antibody at } 20,000 \mathrm{~g} \text { for } 5 \mathrm{~min} \text { at } 4{ }^{\circ} \mathrm{C} \text { and } \\
\text { use the supernatant for Step } 2\end{array}$ \\
\hline & & & $\begin{array}{l}\text { Rotate the device } 180^{\circ} \text { to make use of the other } \\
\text { membrane }\end{array}$ \\
\hline & & & $\begin{array}{l}\text { Use a Vivaspin ultrafiltration device instead, as it can } \\
\text { concentrate antibody down to } 5 \mu \mathrm{l}\end{array}$ \\
\hline \multirow[t]{3}{*}{13} & $\begin{array}{l}\text { Antibody is lost after } \\
\text { ultrafiltration }\end{array}$ & The membrane is damaged & $\begin{array}{l}\text { Check the centrifugal force and avoid touching the } \\
\text { membrane with the pipette tip }\end{array}$ \\
\hline & & $\begin{array}{l}\text { The antibody is binding to the } \\
\text { regenerated cellulose membrane }\end{array}$ & $\begin{array}{l}\text { Use a Vivaspin ultrafiltration device instead, as it uses } \\
\text { polyethersulfone rather than a regenerated cellulose } \\
\text { membrane }\end{array}$ \\
\hline & $\begin{array}{l}\text { Antibody concentration is much } \\
\text { higher than expected }\end{array}$ & $\begin{array}{l}\text { The antibody solution contains } \\
\text { stabilizing proteins such as BSA }\end{array}$ & $\begin{array}{l}\text { Pre-purify the antibody using a } 0.2-\mathrm{ml} \text { Nab Protein } \\
\text { A/G Spin Kit }\end{array}$ \\
\hline
\end{tabular}

Trim-Away by microinjection of mouse oocytes and pre-implantation embryos

\begin{tabular}{|c|c|c|c|}
\hline $16 A($ iv-viii) & $\begin{array}{l}\text { mRNA mix mixes with the } \\
\text { antibody mix within the capillary }\end{array}$ & $\begin{array}{l}\text { The mRNA mix was loaded before the } \\
\text { antibody mix }\end{array}$ & Load the antibody mix before the mRNA mix \\
\hline \multirow{8}{*}{$\begin{array}{l}16 A(x i x) / \\
16 B(i x) / \\
16 C(i i) / \\
16 C(v i)\end{array}$} & $\begin{array}{l}\text { Microinjection needle or } \\
\text { Femtotip II becomes clogged } \\
\text { every few injections }\end{array}$ & $\begin{array}{l}\text { Precipitated materials were loaded into } \\
\text { the capillary }\end{array}$ & Pre-clear for longer and/or break the tip more \\
\hline & & $\begin{array}{l}\text { Precipitated materials were loaded into } \\
\text { the Femtotip II }\end{array}$ & $\begin{array}{l}\text { Pre-clear for longer, break the tip more and/or use the } \\
\text { 'Clean' function }\end{array}$ \\
\hline & & The antibody concentration is too high & $\begin{array}{l}\text { Use } 0.1 \% \text { ( } \mathrm{vol} / \mathrm{vol} \text { ) NP- } 40 \text { and/or inject a larger } \\
\text { volume of diluted antibody mix }\end{array}$ \\
\hline & $\begin{array}{l}\text { Oocytes and early embryos do } \\
\text { not survive the microinjection }\end{array}$ & The $\mathrm{GV}$ is damaged & $\begin{array}{l}\text { Inject close to the GV but do not penetrate the } \\
\text { membrane of the GV }\end{array}$ \\
\hline & & $\begin{array}{l}\text { The opening of the microinjection } \\
\text { needle is too wide }\end{array}$ & $\begin{array}{l}\text { Adjust the program of the needle puller to obtain a } \\
\text { thinner microinjection needle }\end{array}$ \\
\hline & & & $\begin{array}{l}\text { Break the tip of the microinjection needle just enough } \\
\text { for the mercury to flow to the tip }\end{array}$ \\
\hline & & The injection volume is too large & Do not inject more than $12 \mathrm{pl}(\sim 6 \%)$ for mouse oocytes \\
\hline & & $\begin{array}{l}\text { The intracellular NP- } 40 \text { concentration is } \\
\text { too high }\end{array}$ & $\begin{array}{l}\text { Use } 0.05 \% \text { (vol/vol) NP-40 and/or inject less antibody } \\
\text { mix }\end{array}$ \\
\hline
\end{tabular}


Table 1 (continued)

\begin{tabular}{ll} 
Step & Problem \\
\hline $16 \mathrm{~A}(\mathrm{xxi}) /$ & Target protein is not degraded \\
$16 \mathrm{~B}(\mathrm{xi}) /$ & \\
$16 \mathrm{Vii})$ & \\
& $\begin{array}{l}\text { Depletion is not efficient or } \\
\text { complete }\end{array}$
\end{tabular}
complete

Oocytes and early embryos do not develop normally 10- $\mu$ l Neon Tip

\section{Possible reason}

The antibody does not recognize the antigen

TRIM21 does not recognize the antibody

The microinjection was not successful

Oocytes and early embryos were treated with a proteasome inhibitor such as MG132

The endogenous level of the target protein is too high

The expression level of Trim 21 mRNA is too low

The Trim 21 mRNA is degraded

Microinjected oocytes and early embryos were left outside the incubator for too long

The injection volume was too large

The intracellular NP-40 concentration is too high

The mRNA mix and/or antibody mix is/ are contaminated by embryo-toxic substance(s)

The antibody has secondary inhibitory effects

Reporter mRNA(s) is/are

overexpressed

Air bubbles are present in the sample, inaccurate pipetting or insufficient sample volume

The antibody does not recognize the antigen

The antibody does not recognize the native protein inside the cells

The electroporated antibody cannot gain access to the target protein

TRIM21 does not recognize the antibody

Electroporation of the antibody was not successful

Cells were treated with proteasome inhibitor such as MG132

Depletion is not efficient or complete

Cells have low survival rates
The endogenous level of the target protein is too high

TRIM21 levels are too low

Arcing (sparks) occurs during electroporation

Cells were incubated for too long in Neon Buffer R

\section{Solution}

Use another antibody

Confirm that the antibody contains an exposed Fc region or use another antibody

Confirm that the microinjection needle or Femtotip II penetrates the plasma membrane and there is cytoplasmic movement due to liquid outflow

Do not use a proteasome inhibitor during incubation or apply it after incubation

Incubate longer to allow degradation before the start of the assay, use a higher concentration of antibody and/ or inject more antibody mix

Incubate longer to allow expression before the start of the assay, use a higher concentration of Trim 21 mRNA and/or poly(A)-tail Trim21 mRNA

Make sure that all materials are handled in an RNasefree manner

Inject fewer oocytes and early embryos at a time and replace the medium in the microinjection chamber after each round of microinjection

Do not inject more than $12 \mathrm{pl}$ ( $6 \%$ of the oocyte volume) for mouse oocytes

Use $0.05 \%$ ( $\mathrm{vol} / \mathrm{vol}$ ) NP-40 and/or inject less antibody mix

Test the nuclease-free water and/or repeat the mRNA and/or antibody preparation

Reduce the injection volume or antibody concentration and/or use another antibody

Reduce the injection volume and/or mRNA concentration

Gently tap the sample tube to remove air bubbles before taking up sample into the $10-\mu$ l Neon Tip, ensure that the electroporation reaction sample has a volume of $12.5 \mu \mathrm{l}$ or take up the sample into the $10-\mu \mathrm{l}$ Neon Tip more slowly

Use another antibody

Use an antibody that has been tested to work by immunofluorescence

Allow a longer incubation time; incubation of cells for at least one cell division cycle allows degradation of retained nuclear target proteins

Use whole IgG molecules from mammalian hosts

Check the antibody concentration before electroporation; check for arcing (sparks) during electroporation (likely caused by air bubbles in the Neon Tip)

Do not use proteasome inhibitor during Trim-Away

Incubate longer to allow degradation before the start of the assay; use a higher concentration of the antibody Use TRIM21-overexpressing stable cell lines; coelectroporate recombinant TRIM21 protein together with the antibody

Ensure that there are no air bubbles in the $10-\mu \mathrm{l}$ Neon Tip

Proceed to antibody electroporation within $10 \mathrm{~min}$ of resuspending cells in Neon Buffer $\mathrm{R}$ 


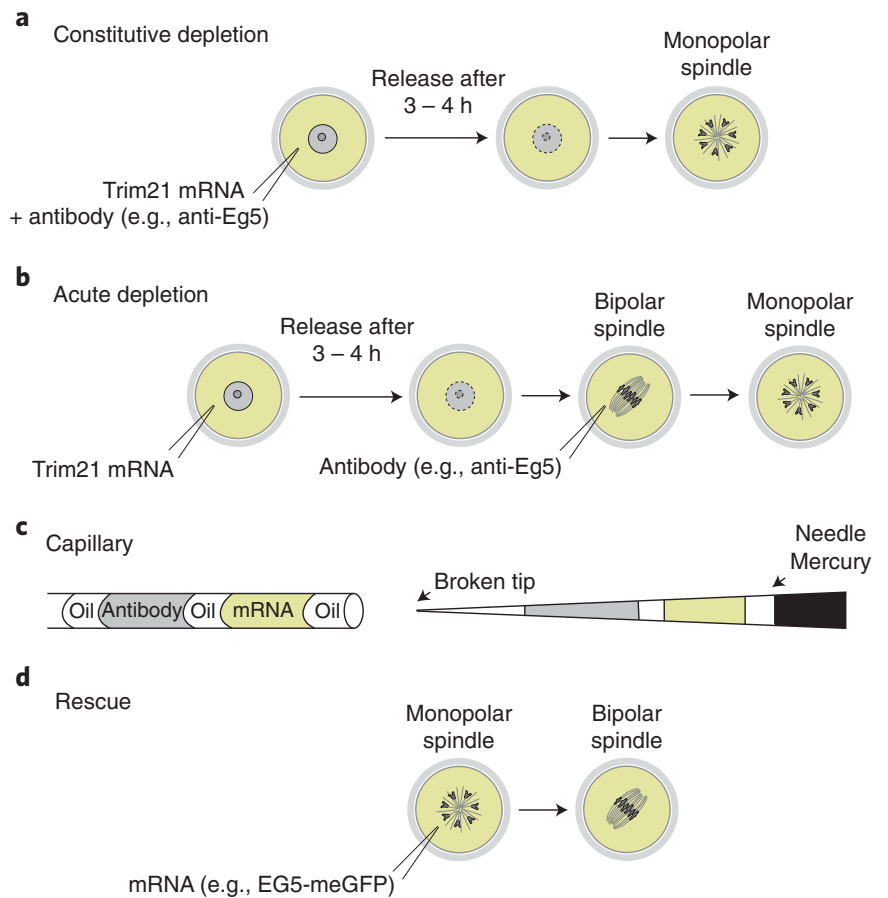

Fig. 3 | Schematic of Trim-Away experiments in mouse oocytes. a, For constitutive depletion, mouse oocytes are co-injected with Trim 21 mRNA and antibody, and the target protein is degraded as Trim 21 mRNA is expressed for 3-4 h before release. $\mathbf{b}$, For acute depletion, mouse oocytes are first injected with Trim21 mRNA, and Trim21 mRNA is expressed for 3-4 h before release. At the desired time point, the antibody is acutely injected into the oocytes. c, For a hydraulic-based microinjection system, the capillary is loaded with the first oil, antibody mix, the second oil, and the mRNA mix, followed by the third oil. The liquids are taken up into the microinjection needle in the reverse order. d, For rescue, mRNA encoding the degraded target protein is acutely injected into the oocytes.

Steps $1-14$, antibody preparation: $1-2 \mathrm{~h}$

Step 15, isolation and culture of mouse oocytes and pre-implantation embryos: $>1 \mathrm{~h}$

Step 16, Trim-Away by microinjection of mouse oocytes and pre-implantation embryos: $4-5 \mathrm{~h}$

Steps 17-32, Trim-Away by electroporation in primary cells and cell lines: $<1 \mathrm{~h}$

Box 3, peptide pre-incubation assay: $2-4 \mathrm{~d}$

Box 4, Trim 21 and reporter mRNA preparation: $8 \mathrm{~h}$

Box 1, preparation of TRIM21 stable cell lines: 2-3 weeks

Box 2, preparation of recombinant TRIM21 protein: $3 \mathrm{~d}$

Generation of TRIM21 stable cell lines as described in Box 1 should result in all cells overexpressing TRIM21 diffusely in the cytoplasm (Fig. 2a,b,d,e). Representative results for Trim-Away assays in cell lines overexpressing TRIM21 are shown in Fig. 2c,f. The expression and purification protocol described in Box 2 should provide TRIM21 protein yields of at least $1 \mathrm{mg} / \mathrm{ml}$ (Fig. $2 \mathrm{~g}$ ). Fig. $2 \mathrm{~h}$ shows Trim-Away of ERK1 in five different unmodified human cell lines by either electroporating antiERK1 antibody alone or together with recombinant TRIM21 protein.

Representative results for a Trim-Away assay in mouse oocytes are shown in Fig. 4. In the example shown, it can be seen that depletion of motor protein kinesin-5 (Eg5) by Trim-Away resulted in a monopolar spindle during meiosis I (MI) (Fig. 4a). This is consistent with inhibition of the motor activity of Eg5 with monastrol ${ }^{45}$. Subsequent expression of Eg5-meGFP 

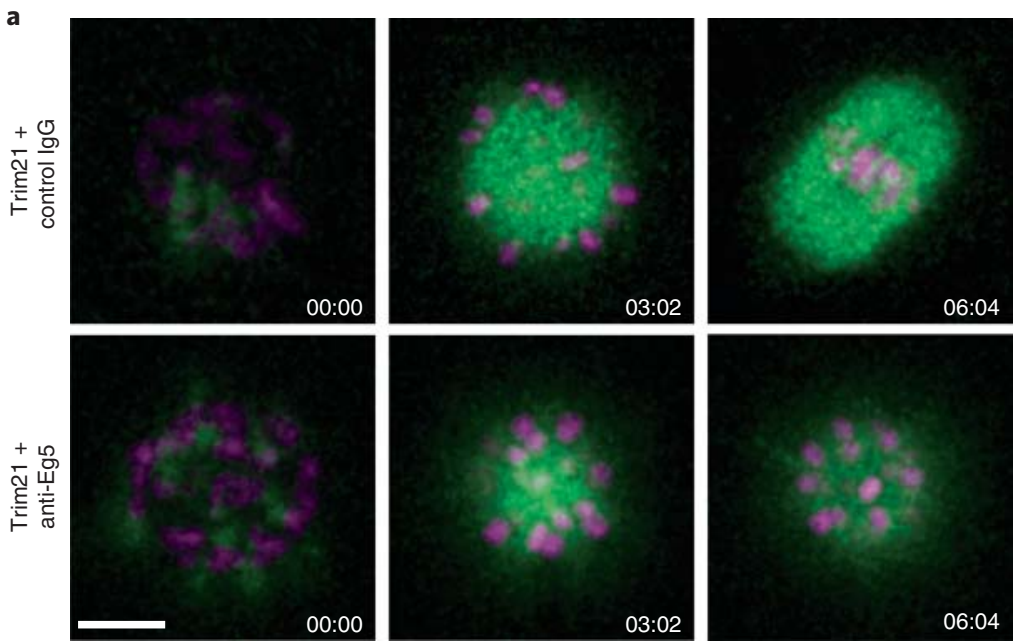

Microtubules

Chromosomes
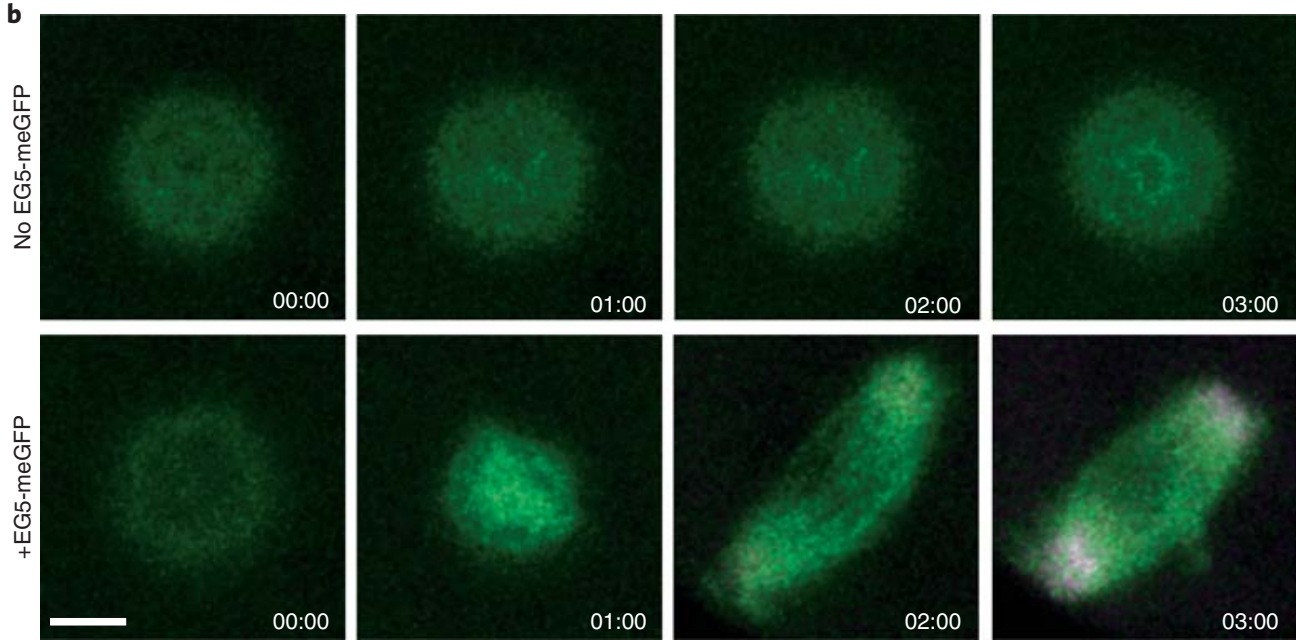

$\square$ Microtubules

$\square$ Chromosomes

Fig. 4 | Trim-Away in mouse oocytes. a, 3D time-lapse imaging of mouse oocytes expressing meGFP-MAP4 (green, microtubules) and H2B-mCherry (magenta, chromosomes), injected with Trim21 mRNA and control lgG or anti-Eg5 (Sigma-Aldrich, cat. no. HPA010568). z-projection, five sections every $6 \mu \mathrm{m}$. Scale bars, $10 \mu \mathrm{m}$. Time is given as hours:minutes from nuclear envelope breakdown (NEBD). b, 3D time-lapse imaging of Eg5-depleted mouse oocytes expressing mCherry-MAP4 (green, microtubules), injected with solvent or EG5-meGFP mRNA. z-projection, five sections every $6 \mu \mathrm{m}$. Scale bars, $10 \mu \mathrm{m}$. Time is given as hours:minutes from NEBD.

from mRNA reverted the phenotype (Figs. $3 \mathrm{~d}$ and $4 \mathrm{~b}$ ), confirming the specificity of Eg5 depletion by Trim-Away.

\section{Reporting Summary}

Further information on experimental design is available in the Nature Research Reporting Summary linked to this article.

\section{References}

1. Gaj, T., Gersbach, C. A. \& Barbas, C. F. ZFN, TALEN and CRISPR/Cas-based methods for genome engineering. Trends Biotechnol. 31, 397-405 (2013).

2. Boettcher, M. \& McManus, M. T. Choosing the right tool for the job: RNAi, TALEN or CRISPR. Mol. Cell 58, 575-585 (2015).

3. Eisen, J. S. \& Smith, J. C. Controlling morpholino experiments: don't stop making antisense. Development 135, 1735-1743 (2008). 
4. Toyama, B. H. et al. Identification of long-lived proteins reveals exceptional stability of essential cellular structures. Cell 154, 971-982 (2013).

5. Rao, P. N. \& Engelberg, J. Mitotic duration and its variability in relation to temperature in HeLa cells. Exp. Cell Res. 52, 198-208 (1968).

6. Weiss, W. A., Taylor, S. S. \& Shokat, K. M. Recognizing and exploiting differences between RNAi and smallmolecule inhibitors. Nat. Chem. Biol. 3, 739-744 (2007).

7. El-Brolosy, M. A. \& Stainier, D. Y. R. Genetic compensation: a phenomenon in search of mechanisms. PLoS Genet. 13, e1006780 (2017).

8. Banaszynski, L. A. et al. A rapid, reversible, and tunable method to regulate protein function in living cells using synthetic small molecules. Cell 126, 995-1004 (2006).

9. Neklesa, T. K. et al. Small-molecule hydrophobic tagging-induced degradeation of HaloTag fusion proteins. Nat. Chem. Biol. 7, 538-43 (2011).

10. Nishimura, K. et al. An auxin-based degron system for the rapid depletion of proteins in nonplant cells. Nat. Methods 6, 917-922 (2009).

11. Caussinus, E., Kanca, O. \& Affolter, M. Fluorescent fusion protein knockout mediated by anti-GFP nanobody. Nat. Struct. Mol. Biol. 19, 117-121 (2011).

12. Robinson, M. S., Sahlender, D. A. \& Foster, S. D. Rapid inactivation of proteins by rapamycin-induced rerouting to mitochondria. Dev. Cell 18, 324-331 (2010).

13. Sakamoto, K. M. et al. Protacs: chimeric molecules that target proteins to the Skp1-Cullin-F box complex for ubiquitination and degradation. Proc. Natl. Acad. Sci. USA 98, 8554-8559 (2001).

14. Fan, X. et al. Rapid and reversible knockdown of endogenous proteins by peptide-directed lysosomal degradeation. Nat. Neurosci. 17, 471-80 (2014).

15. Clift, D. et al. A method for the acute and rapid degradation of endogenous proteins. Cell 171, 1692-1706 (2017).

16. James, L. C. et al. Structural basis for PRYSPRY-mediated tripartite motif (TRIM) protein function. Proc. Natl. Acad. Sci. USA 104, 6200-6205 (2007).

17. Mallery, D. L. et al. Antibodies mediate intracellular immunity through tripartite motif-containing 21 (TRIM21). Proc. Natl. Acad. Sci. USA 107, 19985-19990 (2010).

18. Watkinson, R. E. et al. TRIM21 promotes cGAS and RIG-I sensing of viral genomes during infection by antibody-opsonized virus. PLoS Pathog. 11, e1005253 (2015).

19. McEwan, W. A. et al. Cytosolic Fc receptor TRIM21 inhibits seeded tau aggregation. Proc. Natl. Acad. Sci. USA 114, 574-579 (2017)

20. Hauler, F. et al. AAA ATPase p97/VCP is essential for TRIM21-mediated virus neutralization. Proc. Natl. Acad. Sci. USA 109, 19733-19738 (2012).

21. Fletcher, A. J. et al. Sequential ubiquitination and deubiquitination enzymes synchronize the dual sensor and effector functions of TRIM21. Proc. Natl. Acad. Sci. USA 112, 10014-10019 (2015).

22. Tachibana-Konwalski, K. et al. Rec8-containing cohesion maintains bivalents without turnover during the growing phase of mouse oocytes. Genes Dev. 24, 2505-2516 (2010).

23. Yoshimi, R. et al. Gene disruption study reveals a nonredundant role for TRIM21/Ro52 in NF-kappaBdependent cytokine expression in fibroblasts. J. Immunol. 182, 7527-7538 (2009).

24. Keeble, A. H. et al. TRIM21 is an IgG receptor that is structurally, thermodynamically and kinetically conserved. Proc. Natl. Acad. Sci. USA 105, 6045-6050 (2008).

25. Cromm, P. M. \& Crews, C. M. Targeted protein degradation: from chemical biology to drug discovery. Cell Chem. Biol. 24, 1181-1190 (2017).

26. McEwan, W. A. et al. Intracellular antibody-bound pathogens stimulate immune signaling via the Fc receptor TRIM21. Nat. Immunol. 14, 327-336 (2013).

27. Foss, S. et al. TRIM 21 immune signaling is more sensitive to antibody affinity than its neutralization activity. J. Immunol. 196, 3452-3459 (2016).

28. Bottermann, M. et al. Antibody-antigen kinetics constrain intracellular humoral immunity. Sci. Rep. 6, 37457 (2016).

29. Smoak, E. M. et al. Long-term retention of CENP-A nucleosomes in mammalian oocytes underpins transgenerational inheritance of centromere identity. Curr. Biol. 26, 1110-1116 (2016).

30. Conic, S. et al. Imaging of native transcription factors and histone phosphorylation at high resolution in live cells. J. Cell Biol. https://doi.org/10.1083/jcb.201709153 (2018).

31. Jaffe, L. A. \& Terasaki, M. Quantitative microinjection of oocytes, eggs, and embryos. Methods Cell Biol. 74, 219-242 (2004).

32. Kim, J. A. et al. A novel electroporation method using a capillary and wire-type electrode. Biosens. Bioelectron. 23, 1353-1360 (2008).

33. Freund, G. et al. Targeting endogenous nuclear antigens by electrotransfer of monoclonal antibodies in living cells. MAbs 5, 518-522 (2013).

34. Behringer, R., Gertsenstein, M., Nagy, K. V. \& Nagy, A. Preparation of media for embryo handling and culture. Manipulating the Mouse Embryo 4th edn, Ch. 4, 1 (Cold Spring Harbor Laboratory Press, Cold Spring Harbor, NY, 2014).

35. Behringer, R. et al. Making embryo-handling pipettes from hard glass capillaries. Manipulating the Mouse Embryo 4th edn, Ch. 4, Protocol 3 (Cold Spring Harbor Laboratory Press, Cold Spring Harbor, NY, 2014). 
36. Schuh, M. \& Ellenberg, J. Self-organization of MTOCs replaces centrosome function during acentrosomal spindle assembly in live mouse oocytes. Cell 130, 484-98 (2007).

37. Behringer, R. et al. In vitro fertilization. Manipulating the Mouse Embryo 4th edn, Ch. 15, Protocol 2 (Cold Spring Harbor Laboratory Press, Cold Spring Harbor, NY, 2014).

38. Behringer, R. et al. Collection of zygotes and removal of cumulus cells with hyaluronidase. Manipulating the Mouse Embryo 4th edn, Ch. 4, Protocol 6 (Cold Spring Harbor Laboratory Press, Cold Spring Harbor, NY, 2014).

39. Behringer, R. et al. Collection of two-cell- to morula-stage embryos. Manipulating the Mouse Embryo 4th edn, Ch. 4, Protocol 7 (Cold Spring Harbor Laboratory Press, Cold Spring Harbor, NY, 2014).

40. Behringer, R. et al. Setting up microdrops culture. Manipulating the Mouse Embryo 4th edn, Ch. 4, Protocol 2 (Cold Spring Harbor Laboratory Press, Cold Spring Harbor, NY, 2014).

41. Borrego-Pinto, J., Somogyi, K. \& Lénárt, P. Live imaging of centriole dynamics by fluorescently tagged proteins in starfish oocyte meiosis. Methods Mol. Biol. 1457, 145-166 (2016).

42. Minaschek, G., Bereiter-Hahn, J. \& Bertholdt, G. Quantitation of the volume of liquid injected into cells by means of pressure. Exp. Cell Res. 183, 434-442 (1989).

43. Lee, G. M. Measurement of volume injected into individual cells by quantitative fluorescence microscopy. J. Cell Sci. 94, 443-447 (1989).

44. Tetkova, A. \& Hancova, M. Mouse oocyte isolation, cultivation and RNA microinjection. Bio-protocol 6, e1729 (2016).

45. Clift, D. \& Schuh, M. A three-step MTOC fragmentation mechanism facilitates bipolar spindle assembly in mouse oocytes. Nat. Commun. 6, 7217 (2015).

\section{Acknowledgements}

We thank A. Musacchio and S. Maffini for advice on protein electroporation, and members of the Schuh lab and the James lab for helpful discussions. The research leading to these results received financial support from the Medical Research Council (MC_U105192711 and MC_U105181010), the Max Planck Society, the European Community's Seventh Framework Programme (FP7/2007-2013) under grant agreement no. 241548, European Research Council (ERC) Starting Grant no. 337415 and a Wellcome Trust Investigator Award.

\section{Author contributions}

D.C. and M.S. conceived and designed the study. D.C. carried out all experiments, with the following exceptions: C.S. simplified and optimized the methods for conducting Trim-Away experiments in mouse oocytes and early embryos, and optimized the peptide-binding assay. W.A.M. and D.C. optimized antibody electroporation. W.A.M. generated and characterized stable cell lines. D.C. and L.C.J. purified recombinant TRIM21 protein. C.S. and D.C. prepared figures. L.C.J. wrote the section on antibody purification; W.A.M. wrote the section on generating stable cell lines; and C.S., D.C. and M.S. wrote all other sections of the manuscript. All authors edited the manuscript. M.S. supervised the study.

\section{Competing interests}

The authors declare no competing interests.

\section{Additional information}

Supplementary information is available for this paper at https://doi.org/10.1038/s41596-018-0028-3.

Reprints and permissions information is available at www.nature.com/reprints.

Correspondence and requests for materials should be addressed to D.C., L.C.J. or M.S.

Publisher's note: Springer Nature remains neutral with regard to jurisdictional claims in published maps and institutional affiliations.

Published online: 24 September 2018

Related Link

Key reference using this protocol

1. Clift, D. et al. Cell 171, 1692-1706.e18 (2017) https://doi.org/10.1016/j.cell.2017.10.033 
RESEARCH ARTICLE

OOCYTE DIVISION

\section{A liquid-like spindle domain promotes acentrosomal spindle assembly in mammalian oocytes}

\author{
Chun So ${ }^{1 *}$, K. Bianka Seres ${ }^{1,2,3 *}$, Anna M. Steyer ${ }^{4,5}$, Eike Mönnich' ${ }^{1}$, Dean Clift $^{2}$, \\ Anastasija Pejkovska ${ }^{1}$, Wiebke Möbius ${ }^{4,5}$, Melina Schuh ${ }^{1,2}+$
}

\begin{abstract}
Mammalian oocytes segregate chromosomes with a microtubule spindle that lacks centrosomes, but the mechanisms by which acentrosomal spindles are organized and function are largely unclear. In this study, we identify a conserved subcellular structure in mammalian oocytes that forms by phase separation. This structure, which we term the liquid-like meiotic spindle domain (LISD), permeates the spindle poles and forms dynamic protrusions that extend well beyond the spindle. The LISD selectively concentrates multiple microtubule regulatory factors and allows them to diffuse rapidly within the spindle volume. Disruption of the LISD via different means disperses these factors and leads to severe spindle assembly defects. Our data suggest a model whereby the LISD promotes meiotic spindle assembly by serving as a reservoir that sequesters and mobilizes microtubule regulatory factors in proximity to spindle microtubules.
\end{abstract}

0 nce every menstrual cycle, an oocyte progresses through the first meiotic division to mature into a fertilizable egg. To this end, the oocyte eliminates half of its chromosomes in a small cell, called a polar body. The remaining chromosomes become aligned in the second metaphase spindle, and the egg is released into the fallopian tube, where it can be fertilized. Upon fertilization, the egg completes the second meiotic division, during which it eliminates half of the remaining sister chromatids into the second polar body. Subsequently, the male and female pronuclei form and progress toward each other, and the mitotic divisions of the embryo begin.

However, mammalian embryos frequently develop abnormally, resulting in miscarriages and genetic disorders such as Down syndrome. The major cause for aberrant embryonic development is aneuploidy in the egg, which results from chromosome segregation errors during oocyte meiosis. Unlike somatic cells and male germ cells, oocytes segregate chromosomes with a specialized microtubule spindle that lacks centrosomes (1). Canonical centrosomes consist of a

\footnotetext{
${ }^{1}$ Department of Meiosis, Max Planck Institute for Biophysical Chemistry, 37077 Göttingen, Germany. ${ }^{2}$ Medical Research Council Laboratory of Molecular Biology, Cambridge CB2 OQH, UK. ${ }^{3}$ Bourn Hall Clinic, Cambridge CB23 2TN, UK. ${ }^{4}$ Electron Microscopy Core Unit, Department of Neurogenetics, Max Planck Institute for Experimental Medicine, 37075 Göttingen, Germany. ${ }^{5}$ Cluster of Excellence Nanoscale Microscopy and Molecular Physiology of the Brain (CNMPB), 37073 Göttingen, Germany.

*These authors contributed equally to this work.

†Corresponding author. Email: melina.schuh@mpibpc.mpg.de
}

pair of centrioles surrounded by pericentriolar material and are the main microtubule organizing centers in centrosomal spindles. These ornucleation and form the two poles of mitotic spindles. Oocytes have developed mechanisms to nucleate microtubules independently of centrosomes. For instance, in Drosophila oocytes, the augmin complex and subito (kinesin-6) mediate microtubule nucleation from the spindle poles and spindle equator, respectively, by recruiting the $\gamma$-tubulin complex (2-4). The $\gamma$-tubulin complex resides at the minus ends of microtubules and serves as a template for microtubule nucleation (5). Chromosomes can also serve as sites of microtubule nucleation, as studied most comprehensively in Xenopus egg extracts: They locally activate the small guanosine triphosphatase Ran, which releases spindle assembly factors from inhibitory binding to importins to promote local microtubule assembly (6).

How acentrosomal spindles are organized in mammalian oocytes is still incompletely understood. Despite the absence of centrosomes, mammalian oocytes express many centrosomal proteins (7). Some of these proteins have been mapped to the acentriolar microtubule organizing centers (aMTOCs) (table S1), which functionally replace centrosomes in mouse oocytes $(8,9)$. However, a comprehensive map of centrosomal protein localization in oocytes is lacking. Such a map would not only shed light on meiosis but may also reveal previously unknown functions and subdomains of centrosomes in other cell types. ganelles serve as the major sites of microtubule the roles of centrosomal proteins during oocyte
Results

Identification of the LISD: A liquid-like meiotic spindle domain

We analyzed the localization of 70 centrosomal and spindle-related proteins in mouse metaphase I oocytes (Fig. 1A; fig. S1, A and B; and table S1). We identified several previously unknown aMTOC components, including CEP120, CP110, DISC1, KIF2B, MCRS1, and TOP2A. Of the 17 centriolar proteins that we examined, only CNTROB, CNAP1, and CP110 localized to aMTOCs, consistent with the absence of centrioles in oocytes (1). Proteins that constitute the pericentriolar material of centrosomes mostly localized to aMTOCs. Several centrosome-associated regulatory kinases and their substrates also localized to aMTOCs, and many of the mapped proteins showed enrichment to the overall spindle region.

Unexpectedly, we observed a previously undescribed domain that contains 19 proteins, including centrosomal proteins (AKAP450, CEP170, and KIZ), centriolar satellite proteins (CEP72, PCM1, and LRRC36), minus-end binding proteins (CAMSAP3 and KANSL3), dynein-related proteins (HOOK3, NDE1, NDEL1, and SPDL1) and proteins that control microtubule nucleation and stability (CHC17, chTOG, GTSE1, HAUS6, MCAK, MYO10, and TACC3) (fig. S1, A and B, and table S1). This domain permeated a large region of the spindle and formed prominent spherical protrusions that extended well beyond the spindle poles during late meiosis I as well as in the metaphase II spindle (Fig. 1, A to C) and was conserved in oocytes from other mammalian species (Fig. 1C and fig. S2).

Many of the proteins observed within this domain have been studied extensively in mitosis, yet similar structures have not been reported in somatic cells. To investigate whether the domain is a specific feature of acentrosomal spindles in mammalian oocytes, we depleted centrosomes in somatic cells by centrinone treatment (10) (fig. S3, A and B). Notably, mitotic spindles in centrosome-depleted mouse embryonic fibroblasts did not possess a related domain (fig. S3, $\mathrm{B}$ to $\mathrm{D}$ ). This result suggests that the domain is not a general feature of acentrosomal spindles but is likely exclusive to female meiotic spindles. As we outline in detail below, this domain forms by phase separation and behaves similar to a liquid. We will therefore refer to it as the liquidlike meiotic spindle domain (LISD).

\section{AURA, TACC3, and $\mathrm{CHC17}$ are essential for LISD assembly}

To investigate LISD assembly, we first examined the role of the regulatory kinases aurora $\mathrm{A}$ (AURA), polo-like kinase 1 (PLK1), and polo-like kinase 4 (PLK4), which control spindle assembly in multiple systems, including oocytes (11). Whereas pharmacological inhibition of PLK1 and PLK4 did not affect LISD assembly, inhibition of AURA led to disruption of the LISD (fig. S4, A to E). Of the LISD-associated proteins that we identified, only TACC3 and GTSE1 are known mitotic substrates of AURA (12-15). Specific depletion of endogenous TACC3 by 
A

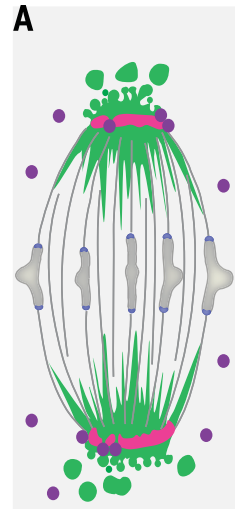

Liquid-like meiotic spindle domain (LISD)

AKAP450

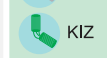

- 、 CEP72

LRRC3

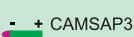

KANSL3

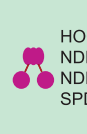

Kinetochores

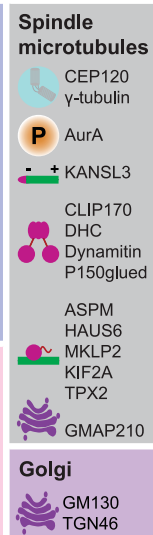

No spindle
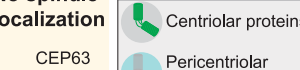
CEP135 material protein CEP152 $\quad$ Regulatory CEP164 Regulatory CETN2 CPAP LRRC45 Ninein NEK2A ODF2 Rootletin
SAS6 SAS6
STIL Acentriolar microtubule-organizing centers (aMTOCs) CNTROB CNAP1
CP110 DISC1
TOP2A
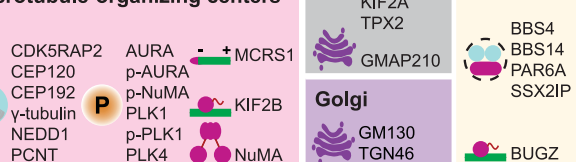

kinases and substrates Other centrosomal proteins - Centriolar satellite Centiolar proteins + Minus-end binding proteins Dynein-related proteins Microtubule related proteins Microtubule , related B $\square \operatorname{LISD}($ TACC 3 )

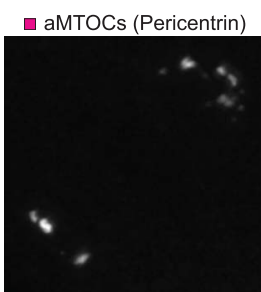

C Mouse

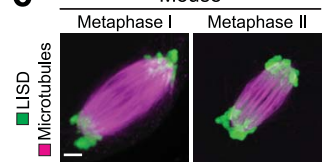
Bovine (cow)

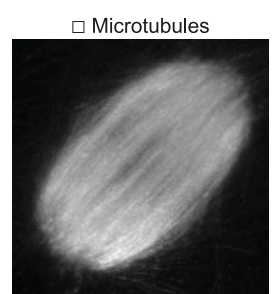

Ovine (sheep)
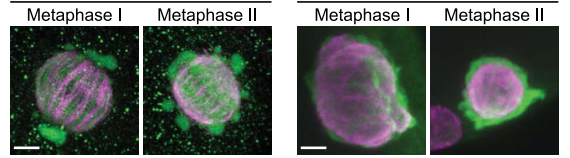

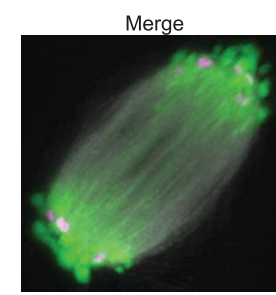

Porcine (pig)

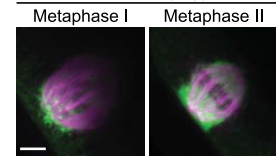

Fig. 1. Identification of a previously unknown spindle domain in mammalian oocytes.

(A) Schematic representation of the mouse metaphase I spindle (scheme; LISD in green, aMTOCs in magenta, kinetochores in blue, Golgi in purple, and spindle microtubules in gray). (B) Immunofluorescence images of a mouse metaphase I spindle. Green, LISD (TACC3); magenta, aMTOCs (pericentrin); gray, microtubules ( $\alpha$-tubulin). (C) Immunofluorescence images of metaphase I and II spindles in mouse, bovine, ovine, and porcine oocytes. Green, LISD (TACC3); magenta, microtubules ( $\alpha$-tubulin). Scale bars, $5 \mu \mathrm{m}$.

Trim-Away fully disassembled the domain (fig. S5, A to D), leading to complete dispersion of multiple LISD-associated proteins, including CAMSAP3 (minus-end stability) (16), chTOG (microtubule nucleation and stability) $(17,18)$, and GTSE1 (microtubule stability) (19, 20) (fig. S6). Some LISD-associated proteins, such as $\mathrm{CHC17}$ (microtubule stability) (21), HAUS6 (microtubule nucleation) $(22,23)$, and KANSL3 (minus-end stability) (24), were no longer detected as prominent protrusions and were strongly depleted from the spindle but still showed some residual association with microtubules upon depletion of TACC3 (fig. S6). By contrast, depletion of GTSE1 had only a minor effect on LISD assembly (fig. S7, A and B). LISD assembly was also disrupted upon depletion of $\mathrm{CHCl} 7$ (fig. S8, A and B), which binds to microtubules together with TACC3 $(25,26)$. TACC3 truncations (26) that are unable to interact with $\mathrm{CHCl} 7$ (fig. S8C) or bind microtubules (fig. S8D) were not incorporated into the LISD, consistent with the codependence of LISD assembly on TACC3 and CHC17. Thus, AURA, TACC3, and $\mathrm{CHC17}$ are essential for LISD assembly.

\section{The LISD is a liquid-like assembly}

To further analyze LISD assembly, we performed live imaging and immunofluorescence on mouse oocytes at different maturation stages. We used TACC3 as the reporter, as it is a core component of the LISD. The LISD accumulated within the center of the microtubule mass after nuclear envelope breakdown (NEBD), permeated the entire microtubule ball as the spindle grew and the aMTOCs fragmented, and translocated toward the two spindle poles together with the aMTOCs during spindle bipolarization (Fig. 2, A and B). As the bipolar spindle increased in size from early to late metaphase I, the LISD grew and extended beyond the spindle poles (Fig. 2, A and B). The LISD was not only enriched in the pole region but also formed projections toward the midzone in the metaphase I spindle (Fig. 1B and movie S1). Protein-retention expansion microscopy and short-term treatment with the EG5 inhibitor monastrol further demonstrated that the LISD was present throughout the spindle body (Fig. 2C and fig. S9), with enrichments on kinetochore fibers (K-fibers) confirmed by coldstable assays and immunoelectron microscopy (Fig. 2C and fig. S10, A to D). Also, the LISD components $\mathrm{CHC17}$ and PCM1 showed similar localization patterns as TACC3 throughout oocyte maturation (fig. S11, A to C).

High-resolution time-lapse microscopy of fluorescently labeled TACC3 further revealed that the spindle pole-associated protrusions of the LISD were dynamic and fused with each other, generating larger protrusions (Fig. 3A). Given that the protrusions of the LISD existed in a region that is largely devoid of microtubules, we explored whether the LISD can be maintained independently of microtubules. Indeed, mouse oocytes acutely treated with the microtubuledepolymerizing drug nocodazole transiently maintained the LISD, even after the bulk of the microtubules had disappeared (Fig. 3B and movie S2). The LISD eventually disappeared and then reassembled into new AURA-dependent spherical condensates after a few minutes (Fig. 3B, fig. S12, and movie S2). Endogenous TACC3 and PCM1 also formed these spherical condensates in oocytes fixed after nocodazole addition (Fig. 3C), demonstrating that they were not an artifact of ectopically expressed, fluorescently labeled TACC3.

Notably, even in the absence of microtubules, the spherical condensates fused with each other and formed larger foci (Fig. 3D and movie S3). This droplet-like behavior was reminiscent of properties of phase-separated structures reported in recent studies (27). We hence investigated whether the LISD might form by phase separation. Phase separation is characterized by the absence of membrane surrounding the phaseseparated structure. To test for the absence of membrane, we established a workflow for focused ion beam-scanning electron microscopy (FIBSEM) of mouse oocytes and imaged the meiotic spindle pole at isotropic resolution in three dimensions (fig. S13A). This analysis confirmed that the LISD was not enclosed by membranes (fig. S13, B and C).

Phase-separated structures are further characterized by their ability to rearrange internally. To test for rearrangement, we performed fluorescence recovery after photobleaching (FRAP) on half of a TACC3-labeled spherical condensate in nocodazole-treated oocytes. The TACC3 signal recovered most prominently directly adjacent to the bleached region, indicating diffusion of fluorescent TACC3 from within the nonbleached region into the bleached region. While the signal recovered in the bleached region, the nonbleached region became dimmer over time, consistent with internal rearrangement within the spherical condensate (Fig. 3E). Consistent with these results, photoactivated bars of TACC3 and PCM1 in intact spindles rapidly spread out bidirectionally within the LISD. By contrast, the photoactivated bar of the microtubule-associated protein TPX2, which is not a LISD component, moved slowly and unidirectionally toward the spindle pole (Fig. 3F). In addition to droplet-like behavior and internal rearrangement, proteins in the LISD and spherical condensates recovered after bleaching (fig. S14, A and B). Notably, proteins in the LISD recovered with similar, rapid kinetics (fig. S14B). By contrast, core aMTOC proteins such as CDK5RAP2, CEP192, and PCNT showed no prominent turnover on aMTOCs in metaphase I spindles (fig. S14C).

Depending on protein dynamics and reversibility, phase-separated structures can be further 


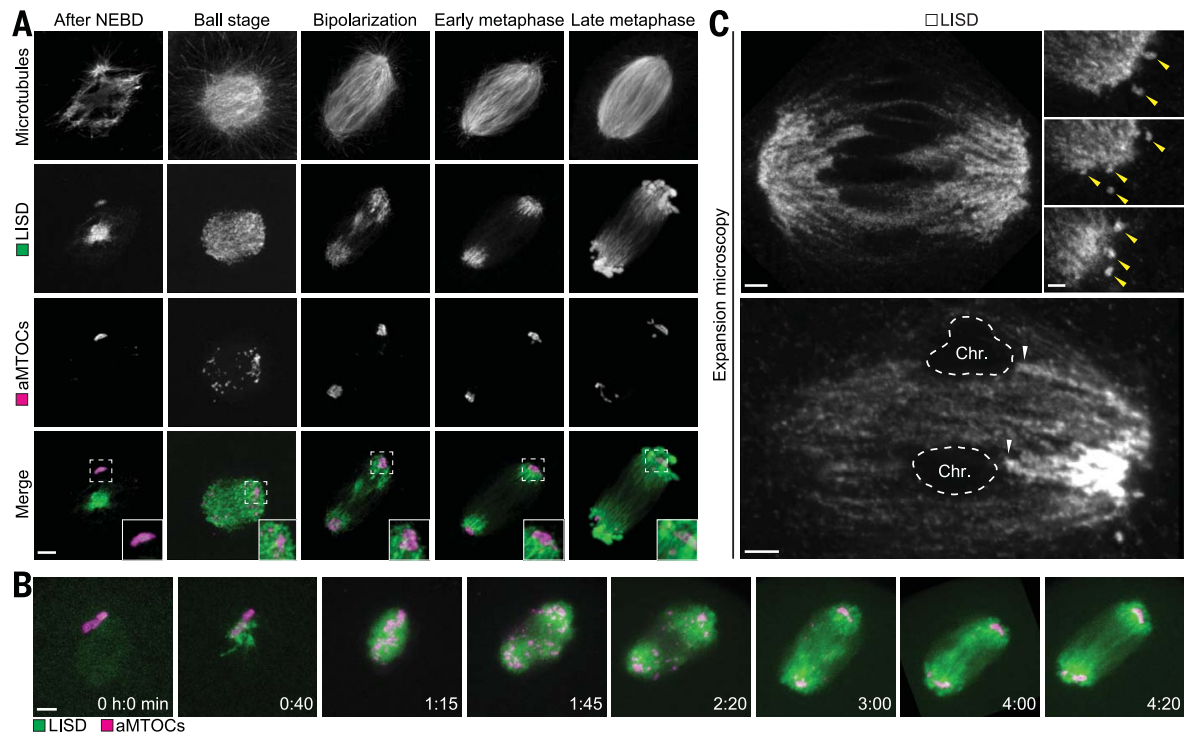

Fig. 2. Dynamics of LISD assembly during meiosis I in mouse oocytes. (A) Immunofluorescence images of mouse oocytes fixed at different times after nuclear envelope breakdown (NEBD). Gray, microtubules ( $\alpha$-tubulin); green, LISD (TACC3); magenta, aMTOCs (pericentrin). Insets are magnifications of regions outlined by dashed boxes. (B) Still images from time-lapse movies of meiotic maturation of mouse oocytes. Green, LISD (TACC3-mClover3); magenta, aMTOCs (CEP192-mScarlet). Time is given as hours:minutes after NEBD. (C) Single sections of expansion microscopy of a mouse early metaphase I spindle. Gray, LISD (TACC3). Yellow arrowheads highlight spherical protrusions at the spindle poles; white arrowheads highlight the K-fibers. Chr.

chromosome. Scale bars, $5 \mu \mathrm{m}$.

classified into liquid-like (dynamic and reversible), gel-like (arrested and reversible), and solidlike (arrested and irreversible) categories (28). As proteins in the LISD were highly dynamic (fig. S14B) and the LISD reversibly disassembled and reassembled upon nocodazole addition and washout (Fig. 2B and fig. S15A), we hypothesized that the LISD is liquid-like. To test this hypothesis further, we discriminated liquid-like from solid-like condensates in oocytes by treating them with 1,6-hexanediol, which dissolves liquid-like condensates by disrupting weak hydrophobic interactions (29). The spherical condensates in nocodazoletreated oocytes dispersed within minutes of 1,6-hexanediol addition (Fig. 3G). 1,6-hexanediol treatment also strongly depleted the LISD on intact spindles, though not completely (fig. S15, $\mathrm{B}$ and C). Finally, thioflavin T, which stains the amyloid-like interactions that are characteristic of solid-like condensates, did not stain the LISD (fig. S15D). Together, these data strongly suggest that the LISD is a liquid-like condensate.

\section{TACC3 phase-separates via its $\mathbf{N}$ terminus}

We discovered that the spherical condensates in nocodazole-treated oocytes were dependent on TACC 3 but only mildly affected by $\mathrm{CHC1} 7$ depletion (fig. S16, A and B). We thus tested whether TACC3 can phase-separate on its own in vitro. In the presence of the macromolecular crowding agent polyethylene glycol (PEG), recombinant TACC3 self-assembled into micrometer-sized spherical droplets over a range of $\mathrm{pH}$ values, ionic strengths, PEG concentrations, and protein concentrations (fig. S17, A to C). TACC3 droplets were able to wet glass surfaces, fuse into larger droplets, and exclude fluorescently labeled $70-\mathrm{kDa}$ dextran (Fig. 4, A to C), consistent with characteristics of phase separation (27). In further support of liquid-liquid phase separation, TACC3 droplets rapidly recovered after bleaching (Fig. 4D and fig. S17D).

In silico predictions suggested that both human and mouse TACC3 are bipartite, with a disordered $\mathrm{N}$ terminus and a structured, coiledcoil-containing $\mathrm{C}$ terminus (Fig. 4E). Both disordered and coiled-coil domains have been implicated in driving phase separation (30). To address which region of TACC 3 mediates phase separation, we purified TACC3 fragments that contained only the coiled-coil TACC domain [amino acids (aa) 594 to 838] or the rest of the protein TACC3( $\triangle \mathrm{TACC}$ ) (aa 1 to 593 ). TACC3 ( $\triangle \mathrm{TACC})$ retained the ability to form droplets, similar to full-length TACC3 (Fig. 4F). By contrast, the TACC domain self-organized into networklike structures (Fig. 4F). These results reveal that the previously uncharacterized disordered $\mathrm{N}$ terminus of TACC3 is necessary and sufficient for phase separation in vitro.

Although the $\mathrm{N}$ terminus of TACC3 is required for its phase separation in vitro, it is dispensable for TACC3 localization in Xenopus mitotic extracts (15) and in mitotic cells (26). To determine whether the TACC3 $\mathrm{N}$ terminus is essential for LISD assembly, we expressed TACC $3(\triangle \mathrm{NT})$ (aa 522 to 838 ) in TACC3-depleted oocytes.
TACC $3(\triangle N T)$ lacks the disordered $\mathrm{N}$ terminus but still contains the regions required to bind both $\mathrm{CHC17}$ and microtubules. Although TACC $3(\Delta \mathrm{NT})$ localized properly to the spindle and associated with $\mathrm{K}$-fibers, as confirmed by cold treatment, it failed to restore LISD assembly (Fig. 4, G and H). Instead, consistent with the behavior of the purified TACC domain in vitro (Fig. $4 \mathrm{~F}), \mathrm{TACC} 3(\Delta \mathrm{NT})$ and $\mathrm{CHCl} 7$ assembled network-like structures at the spindle poles in vivo (Fig. 4G). These data indicate that the $\mathrm{N}$ terminus of TACC3 is essential for phase separation in vivo. Notably though, the $\mathrm{N}$ terminus of TACC3 is not sufficient for TACC3 recruitment to the LISD in vivo, as TACC3 $\triangle \mathrm{TACC}$ ) was not incorporated into the LISD (fig. S8D). Microtubule binding via the TACC domain is apparently required for LISD formation and may help to stabilize the condensates in vivo, as also suggested by the higher resistance of TACC3 condensates to 1,6-hexanediol treatment on intact spindles than in nocodazoletreated oocytes (Fig. 3G and fig. S15, B and C).

\section{The LISD promotes acentrosomal spindle formation by sequestering microtubule regulatory factors}

Phase separation can selectively enrich factors to promote reactions or storage (27). Recent studies proposed that the phase-separated Xenopus microtubule-binding protein BuGZ, regulatory kinase PLK4, and the Caenorhabditis elegans centrosomal protein SPD-5 promote microtubule nucleation in centrosomal spindles by locally enriching tubulin dimers by a factor of 4 to 10 (31-33). By contrast, LISD protrusions and spherical condensates did not significantly concentrate tubulin dimers (fig. S18, A to E). However, microtubule dynamics were altered in TACC3depleted oocytes: Microtubule growth rates were significantly decreased, and their overall turnover was significantly increased (fig. S19, A to D). We thus hypothesized that the LISD locally sequesters microtubule regulatory factors to promote spindle assembly. To investigate this hypothesis, we examined acentrosomal spindle assembly upon ablation of the LISD by three different means: inhibition of AURA, depletion of TACC 3 , and depletion of $\mathrm{CHCl}$, all of which are essential for LISD assembly (see above). Under all conditions, the total microtubule intensity and spindle volume were severely reduced, to about half of the values in control oocytes (Fig. 5, A to C; figs. S20, A to E, and S21, A to I; and movies S4 and S5). Moreover, aMTOCs were no longer scattered around the spindle poles but coalesced into two single foci (figs. S22, A to K, and S23, A to I). Similarly, AURA inhibition and TACC3 depletion in bovine oocytes caused a severe reduction in microtubules as well as spindle assembly defects (Fig. 5, D to F, and fig. S24), in line with the abnormal spindles observed in a previous RNA interference (RNAi) study (34).

In mitotic cells, depletion of TACC3 causes a minor loss of spindle microtubules $(17,23)$ but results in misaligned chromosomes and metaphase arrest $(25,35-38)$. To uncover LISD-specific functions, we took advantage of TACC $3(\Delta \mathrm{NT})$, 
A

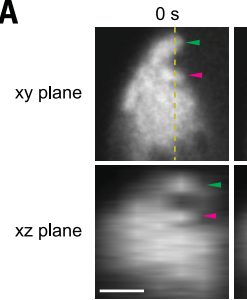

B Acute nocodazole addition

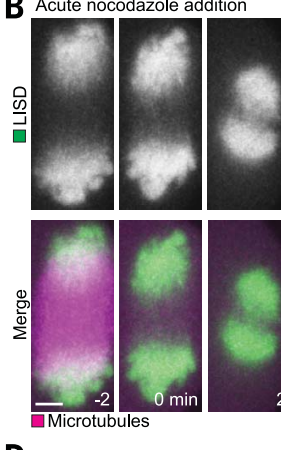

D

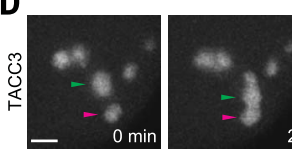

E Pre-bleach Post-bleach Recovered

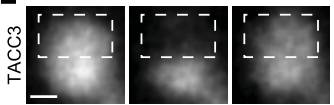

$\mathbf{F}$
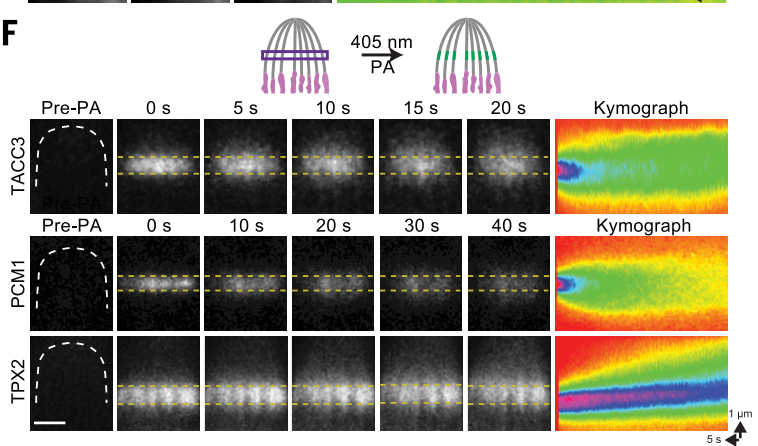

Fig. 3. The LISD forms by phase separation. (A) Stills from time-lapse movies of mouse late metaphase I oocytes. Gray, LISD (TACC3-mClover3). Yellow lines mark the position of $x z$ planes on the corresponding $x y$ planes. Arrowheads highlight fusing LISD protrusions. (B) Still images from time-lapse movies of acutely nocodazole-treated mouse metaphase I oocytes. Green, LISD (TACC3mClover3); magenta, microtubules (EB3-3×mCherry). Time is given as minutes after $10 \mu \mathrm{M}$ nocodazole addition. (C) Immunofluorescence images of spherical condensates in acutely $10 \mu \mathrm{M}$ nocodazole-treated mouse metaphase I oocytes. Gray, microtubules ( $\alpha$-tubulin); green, spherical condensates (TACC3 or PCM1); magenta, aMTOCs (pericentrin). (D) Still images from time-lapse movies of acutely $10 \mu \mathrm{M}$ nocodazole-treated mouse metaphase I oocytes. Gray, spherical condensates (TACC3-mClover3). Arrowheads highlight fusing spherical condensates. (E) Partial bleaching of TACC3-mClover3 in a spherical condensate in acutely $10 \mu \mathrm{M}$ nocodazole-treated mouse metaphase I oocytes. The bleached area is outlined by the dashed box. Scale bar, $1 \mu \mathrm{m}$. (F) Photoactivation (PA) of different proteins on mouse metaphase I spindles. Spindle poles are outlined with white dashed lines; photoactivated bars are marked with yellow dashed lines. Time is given as minutes after photoactivation with a 405-nm laser. (G) Still images from time-lapse movies of acutely 1,6-hexanedioltreated mouse metaphase I oocytes pretreated with $10 \mu \mathrm{M}$ nocodazole. Time is given as minutes after 3.5\% 1,6-hexanediol addition. Scale bars, $5 \mu \mathrm{m}$ unless otherwise specified.

which can neither phase-separate in vitro nor restore LISD assembly in vivo (Fig. 4G). We do not exclude the possibility that there are additional, phase separation-independent functions associated with the $\mathrm{N}$ terminus of TACC3.
However, TACC3 $(\triangle \mathrm{NT})$ has been shown to rescue the phenotypes induced by TACC3 depletion in Xenopus mitotic extracts and in mitotic cells, and the $\mathrm{N}$ terminus of TACC3 has hence been suggested to be dispensable for TACC3 function in these systems $(15,26)$. Notably, expression of TACC3 $(\triangle \mathrm{NT})$ in TACC3-depleted mouse oocytes did not rescue the reduction in total microtubule intensity, and the spindle volume was still significantly reduced compared with that in wild-type oocytes, albeit not as strongly as in TACC3-depleted oocytes, as microtubules in the spindle appeared less densely packed and more spread out (Fig. 6, A to C, and movie S6). Moreover, spindle bipolarization was severely delayed in TACC3( $\triangle \mathrm{NT})$-TACC3-depleted oocytes relative to wild-type oocytes, and spindles progressed through a prolonged phase of spindle instability and fragmentation before becoming bipolar (Fig. 6A and fig. S25, A and B). Also the association between aMTOCs and spindles in TACC3( $\triangle \mathrm{NT})$-TACC3-depleted oocytes was abnormal (fig. S25C). By contrast, TACC3( $\triangle \mathrm{NT})$ rescued progression into anaphase and markedly reduced lagging chromosomes in TACC3depleted oocytes, demonstrating that the construct is functional (fig. S25, D to G). Together, these data establish that the LISD is required for efficient microtubule assembly and to form stable acentrosomal spindles.

\section{K-fibers and interpolar microtubules are depleted upon LISD disruption by TACC3 depletion}

To further investigate the role of the LISD in spindle assembly, we examined K-fibers and interpolar microtubules. To assess K-fibers, we briefly placed oocytes on ice, which depolymerizes dynamic microtubules within the spindle while preserving the more stable, kinetochorebound microtubules. K-fibers were significantly diminished in TACC3-depleted oocytes (Fig. 7, A to C). To assess interpolar microtubules, we treated oocytes with calcium, which preserves both K-fibers and stable interpolar microtubules in mouse oocytes (39). Stable interpolar microtubules were strongly depleted in TACC3-depleted oocytes (Fig. 7D and movies S7 and S8). Quantification revealed a more severe microtubule loss than in the cold-stable assay (Fig. 7, E and F). We also labeled spindles with the microtubule cross-linker PRC1, which associates with interpolar microtubules (40). Consistent with the calciumstable assay, PRC1-marked microtubules were drastically reduced in TACC3-depleted oocytes (Fig. 7, G and H, and movies S9 and S10). In addition, oocytes in anaphase displayed a significant reduction in the total intensity and volume of the central spindle (fig. S26, A to C), which consists of interpolar microtubules and is largely devoid of K-fibers (41). Thus, both K-fibers and interpolar microtubules are depleted upon LISD disruption by TACC3 depletion.

\section{Discussion}

Our data uncover a previously unknown principle of acentrosomal spindle assembly in mammalian oocytes: Meiotic spindle assembly is facilitated by a prominent liquid-like domain that contains multiple microtubule regulatory factors and enriches them in a dynamic manner in proximity to spindle microtubules. The domain 
Fig. 4. The $\mathbf{N}$ terminus of TACC 3 is necessary for phase separation in vitro and in vivo. (A) Bright-field and fluorescence images of GST-TACC3 droplets in vitro. Inset is the magnification of the region marked by the dashed line box. (B) Still images from time-lapse movies of fusing GST-TACC3 droplets in vitro. Scale bar, $1 \mu \mathrm{m}$. Arrowheads highlight fusing GST-TACC3 droplets. (C) Fluorescence images of GST-TACC 3 droplets in the presence of 70-kDa dextran in vitro. Green, GST-TACC3; magenta, 70-kDa dextran. Insets are magnifications of regions marked by dashed line boxes. Scale bar, $2.5 \mu \mathrm{m}$. (D) FRAP of GST-TACC3 droplets in vitro. Gray, GST-TACC3.

The number of analyzed droplets is specified in italics. M.F., mobile fraction. Scale bar, $1 \mu \mathrm{m}$. (E) Domain organization of human and mouse TACC3 showing the disordered region [purple; analysis with DisEMBL (100)] and the coiled-coil domain [yellow; analysis with MARCOIL (101)]. (F) Fluorescence images of GST, GST-TACC3, GST-TACC3( $\triangle$ TACC), and GST-TACC in vitro. (G and

H) Immunofluorescence images of the metaphase I spindle in control, TACC3-depleted, and TACC3( $\triangle \mathrm{NT})$-TACC3depleted mouse oocytes with and without cold treatment. IgG, immunoglobulin G. Insets are magnifications of regions marked by dashed line boxes. All in vitro assays were performed in $\mathrm{pH} 6.4$ buffer with $150 \mathrm{mM} \mathrm{KCl}$ and 12\% PEG. Scale bars, $5 \mu \mathrm{m}$ unless otherwise specified.
A
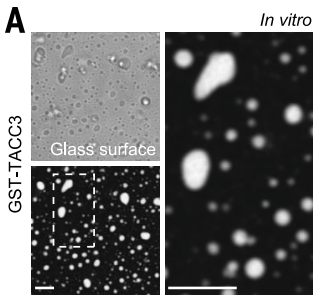

B
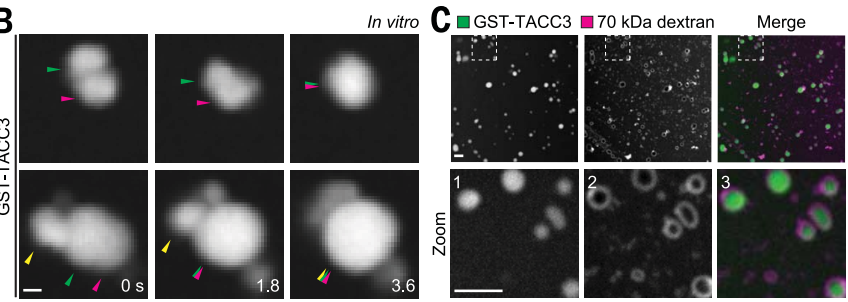

D

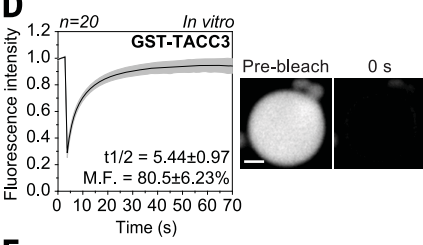

$\mathbf{E}_{1.0}$
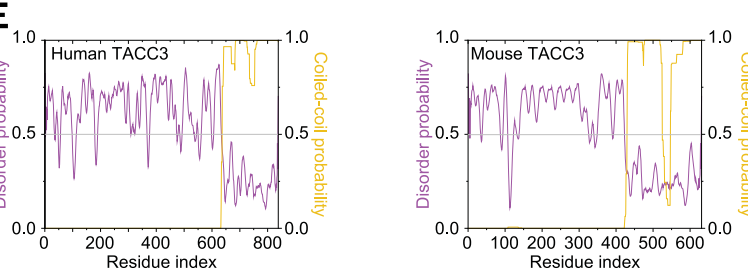

G

H

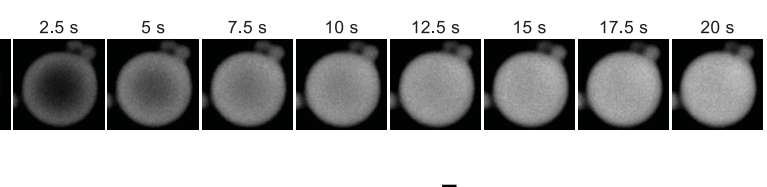

$\mathbf{F}$
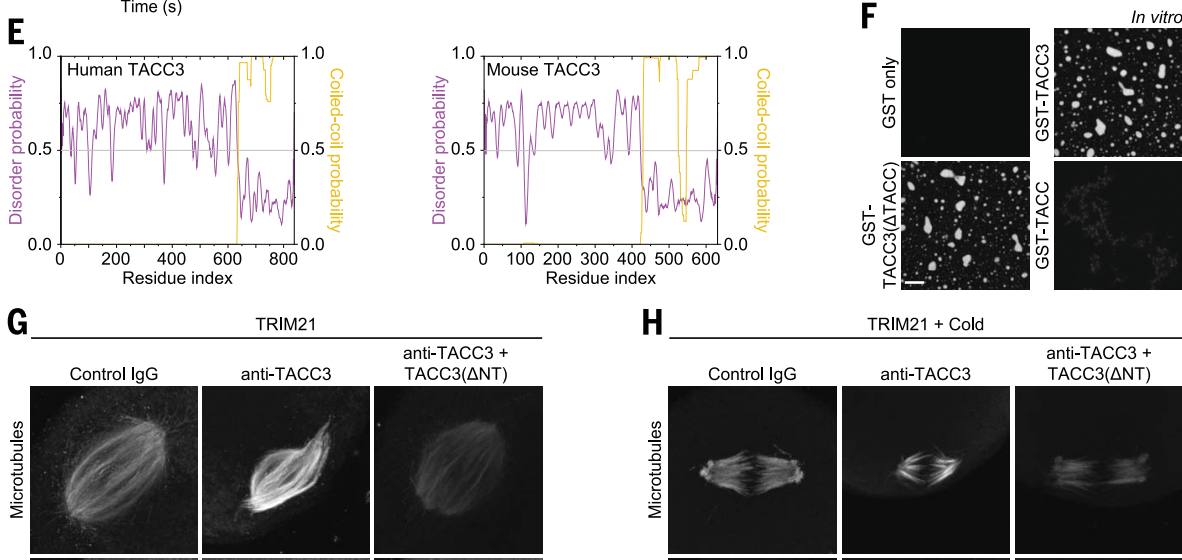

TRIM21
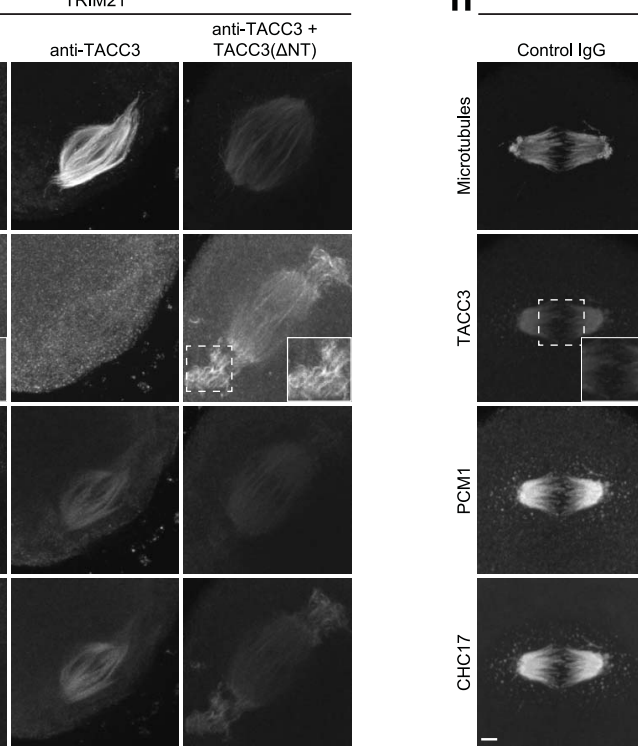

TRIM21 + Cold

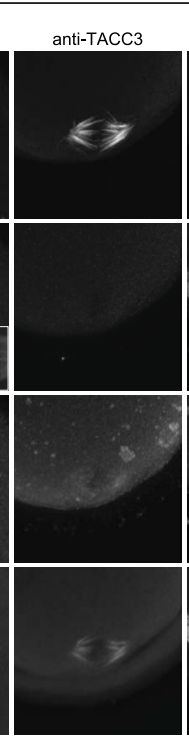

anti-TACC3 + anti-TACC3+
TACC3( $\triangle N T)$

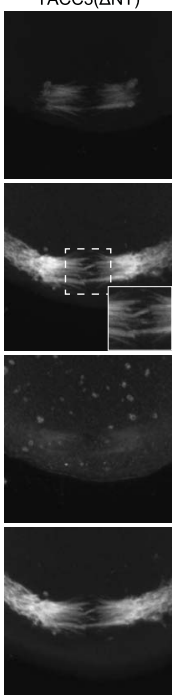

permeates the spindle poles and forms dynamic protrusions extending outward well beyond the spindle region in mouse, bovine, ovine, and porcine oocytes. Ablating the domain results in microtubule loss and defective spindle assembly.

Enriching microtubule regulatory factors in local proximity to the spindle may be particularly important for spindle assembly in large cells such as oocytes, where they would otherwise be dispersed throughout the large cytoplasmic volume. Liquid-liquid phase separation may be an ideal principle for this purpose: It sequesters factors within proximity to microtubules but still allows them to diffuse dynamically throughout the spindle region. This could also help to control the local concentration of certain spindle-related factors and prevent them from accumulating within specific spindle regions such as the spin- dle poles or kinetochores but may instead promote their even distribution throughout the entire spindle volume.

Does the LISD play a role in spindle assembly beyond mammalian oocytes? Structures that resemble the LISD have not yet been reported in other cell types (42), although many LISD proteins have been extensively studied in mitotic cells. Given the absence of LISD-related structures in centrosome-depleted mitotic cells, it appears likely that the LISD is an oocyte-specific structure. Notably though, when TACC3 is highly overexpressed in somatic cells, it forms large structures that can associate with the mitotic spindle (43). Whether phase separation also plays a role in the formation of these structures is unclear.

In centrosomal spindles, phase separation has been proposed to enrich tubulin dimers to facil- itate microtubule nucleation (31-33). However, the LISD does not concentrate tubulin dimers but enriches different microtubule regulatory factors in acentrosomal spindles. One could speculate that an enrichment of tubulin dimers is less important in large oocytes, as tubulin is not limiting in large cytoplasmic volumes $(44,45)$. Indeed, recent studies imply microtubule dynamics as a key factor in determining spindle size when tubulin is not limiting (46). Mammalian oocytes may use phase separation of microtubule regulatory factors as a mechanism to modulate microtubule dynamics and to thereby promote the assembly of large meiotic spindles. The LISD differs from other phase-separated structures in terms of morphology, composition, and mechanism of action, underscoring that phase separation is a widely used principle that can promote 
A
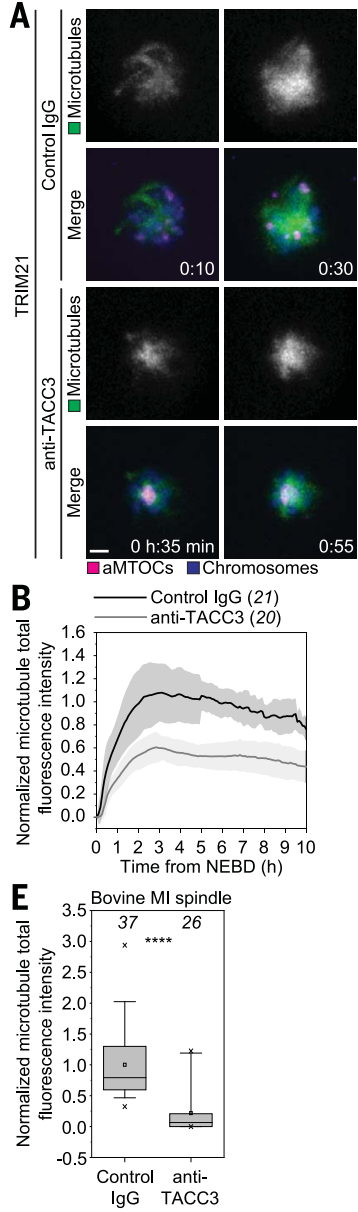
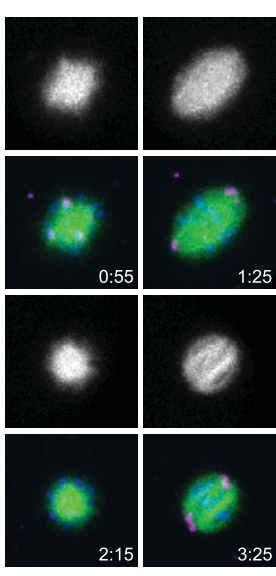

C
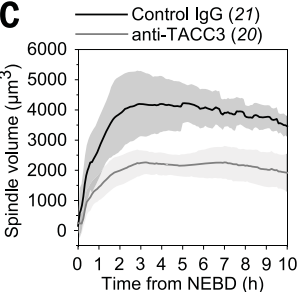

Time from NEBD $(\mathrm{h})$
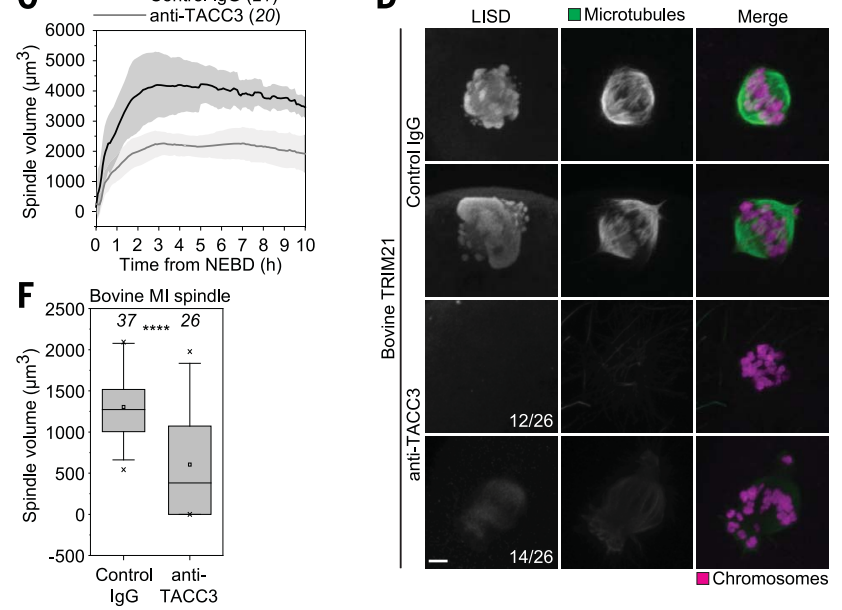

D

Fig. 5. Microtubule loss and defective spindle assembly in TACC3-depleted oocytes. (A) Stills from time-lapse movies of control and TACC3-depleted mouse oocytes. Green, microtubules (mClover3-MAP4-MTBD); magenta, aMTOCs (CEP192-mScarlet); blue, chromosomes (H2B-miRFP) Time is given as hours:minutes after NEBD. (B and $\mathbf{C}$ ) Quantification of total fluorescence intensity of microtubules and spindle volume in control and Tacc3-depleted mouse oocytes. (D) Immunofluorescence images of the metaphase I spindle in control and TACC3-depleted bovine oocytes. Gray, LISD (TACC3); green, microtubules ( $\alpha$-tubulin); magenta, chromosomes (Hoechst). ( $E$ and $\mathbf{F}$ ) Quantification of total fluorescence intensity of microtubules and spindle volume in control and TACC3-depleted bovine metaphase I (MI) oocytes. The number of analyzed oocytes is specified in italics. Error bars (shaded areas) represent SD. Scale bars, $5 \mu \mathrm{m}$.

spindle assembly via distinct mechanisms in different species and cell types.

\section{Materials and methods \\ Preparation and culture of mouse oocytes and follicles}

All mice were maintained in a specific pathogenfree environment according to animal ethics guidelines of the Animal Facility of the Max Planck Institute for Biophysical Chemistry and U.K. Home Office regulations.

Oocytes were isolated from ovaries of 8- to 12 -week-old FVB/N or CD1 female mice. For some experiments that required large numbers of germinal vesicle (GV) oocytes, the mice were primed with $7.5 \mathrm{IU}$ of pregnant mare serum gonadotropin 48 hours before isolation. Fully grown oocytes of $\sim 75 \mu \mathrm{m}$ in diameter with a centered GV were maintained at prophase arrest in homemade phenol red-free M2 medium sup- plemented with $250 \mu \mathrm{M}$ dibutyryl cyclic AMP (dbcAMP) (Sigma-Aldrich) under paraffin oil (ACROS Organics) at $37^{\circ} \mathrm{C}$.

Follicles were isolated from 12-day-old (C57BL $\times$ CBA) F1 female mice as previously described (47) with some minor modifications. Briefly, compact follicles of $\sim 100 \mu \mathrm{m}$ in diameter with a centered oocyte were cultured in MEM-alpha with GlutaMax (Gibco) supplemented with 5\% fetal bovine serum (FBS) (Gibco), $0.01 \mu \mathrm{g} / \mathrm{ml}$ ovine follicle stimulating hormone (National Hormone and Peptide Program), $1 \times$ insulin/ transferrin/sodium selenite (Sigma-Aldrich), and $0.1 \times$ penicillin G/streptomycin (Gibco) on collagen-coated inserts (Corning) at $37^{\circ} \mathrm{C} / 5 \% \mathrm{CO}_{2}$. Medium surrounding the insert was replaced every 3 days. After 8 days of culture, in vitro grown oocytes were denuded and matured in modified M2 medium with $10 \%$ FBS instead of $4 \mathrm{mg} / \mathrm{ml}$ bovine serum albumin (BSA).
Preparation and culture of bovine, ovine, and porcine oocytes

All ovaries were obtained from local slaughterhouses. Bovine ovaries were transported to the laboratory within 2 hours of retrieval in a thermoflask. Ovine and porcine ovaries were transported to the laboratory within 1 hour of retrieval in a portable $37^{\circ} \mathrm{C}$ incubator in M2 medium supplemented with $1 \mathrm{mM}$ dbcAMP. Oocytes were recovered by aspiration of antral follicles with an 18-gauge needle affixed to a 1-ml syringe. For bovine oocytes, $140 \mu \mathrm{l}$ of $5000 \mathrm{IU} / \mathrm{ml}$ heparin (Merck Millipore) was added to every $20 \mathrm{ml}$ of aspirates. Cumulus-oocyte complexes (COCs) were allowed to sediment and then washed extensively with HEPES-buffered medium 199 (for bovine oocytes) or M2 medium (for ovine and porcine oocytes) supplemented with $1 \mathrm{mM}$ dbcAMP. Only fully grown oocytes with a homogeneous cytoplasm and several layers of compact cumulus cells were selected for experiments. Bovine and ovine/porcine oocytes were maintained in prophase arrest in dbcAMP-containing medium at $39^{\circ}$ and $37^{\circ} \mathrm{C}$, respectively. Surrounding cumulus cells of bovine and ovine/porcine oocytes were removed by vortexing and with $30 \mathrm{nM}$ hyaluronidase (Sigma-Aldrich), respectively. Ovine and porcine oocytes were released into dbcAMP-free M2 medium to resume meiosis.

\section{Cell culture}

NIH3T3 cells (ATCC) were cultured in highglucose DMEM supplemented with GlutaMAX, pyruvate, and $10 \%$ calf serum (Gibco). To deplete centrosomes, cells were treated with $300 \mathrm{nM}$ centrinone (Tocris Bioscience) for 12 days. To obtain mitotic spindles, cells were synchronized with $10 \mu \mathrm{M}$ RO-3306 (Tocris Bioscience) for 1 day and then released into RO-3306-free medium for $45 \mathrm{~min}$.

\section{Immunofluorescence}

To obtain mouse and ovine/porcine metaphase I spindles, oocytes were incubated at $37^{\circ} \mathrm{C}$ for around 7 and 12 hours, respectively, upon release into dbcAMP-free medium. To obtain bovine metaphase I spindles, oocytes were incubated at $39^{\circ} \mathrm{C} / 5 \% \mathrm{CO}_{2}$ for $\sim 12$ hours upon release into dbcAMP-free medium. To obtain mouse, bovine, ovine and porcine metaphase II spindles, oocytes were incubated for $\sim 16$ hours upon release into dbcAMP-free medium. None of the oocytes used for immunofluorescence analyses was subjected to live imaging before fixation.

Oocytes and cells were fixed in $100 \mathrm{mM}$ HEPES (pH 7.0, titrated with $\mathrm{KOH}$ ), 50 mM EGTA (pH 7.0, titrated with $\mathrm{KOH}$ ), $10 \mathrm{mM} \mathrm{MgSO}_{4}, 2 \%$ methanolfree formaldehyde, and $0.5 \%$ triton $\mathrm{X}-100$ at $37^{\circ} \mathrm{C}$ for 15 to $60 \mathrm{~min}$. Fixed oocytes and cells were extracted in phosphate-buffered saline (PBS) with $0.5 \%$ triton X-100 (PBT) overnight at $4{ }^{\circ} \mathrm{C}$ and blocked in PBT with 5\% BSA (PBT-BSA) overnight at $4^{\circ} \mathrm{C}$. All antibody incubations were performed in PBT-BSA at $10 \mu \mathrm{g} / \mathrm{ml}$ overnight at $4^{\circ} \mathrm{C}$ (for primary antibodies) or at $20 \mu \mathrm{g} / \mathrm{ml}$ for 1 hour at room temperature (for secondary antibodies). Primary antibodies used were human 
A
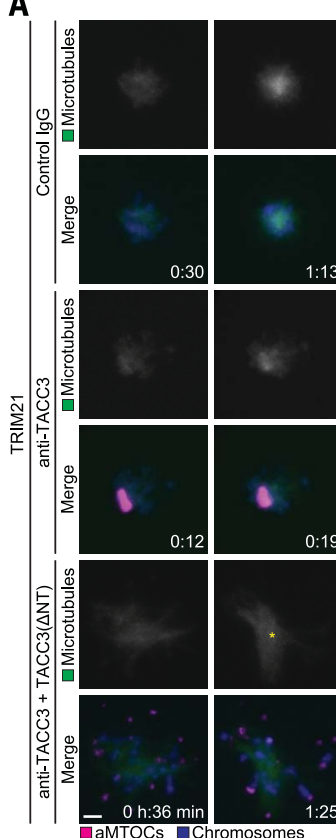
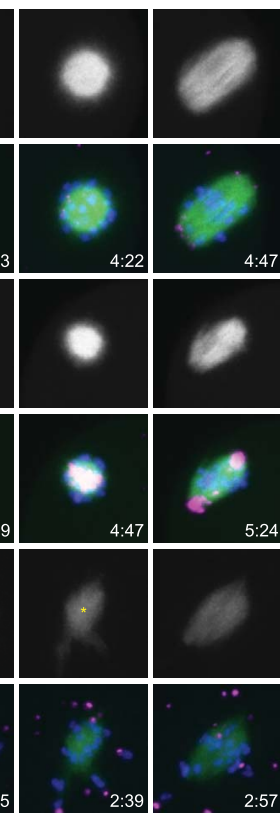
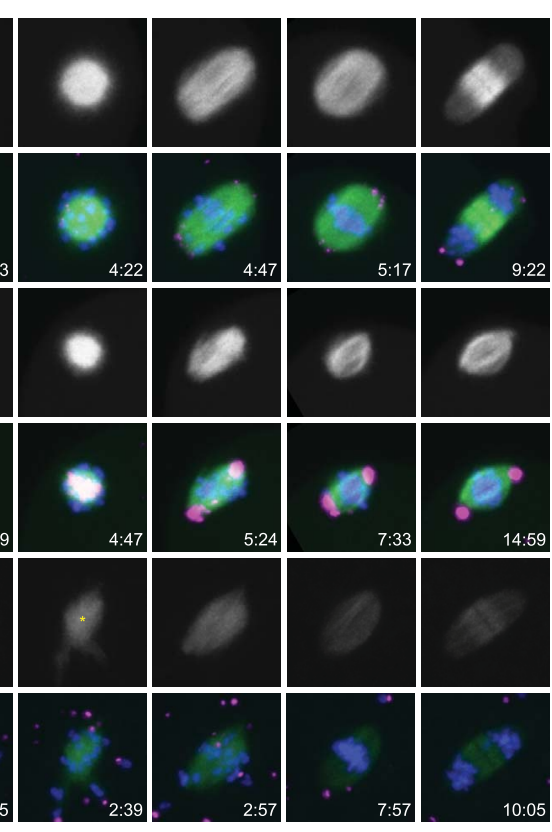

B
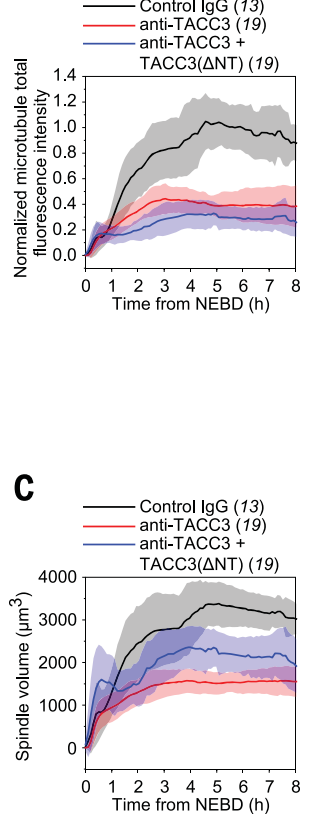

Fig. 6. The $\mathbf{N}$ terminus of TACC3 is required for spindle assembly in mouse oocytes. (A) Still images from time-lapse movies of control, TACC3-depleted, and TACC3( $\triangle N$ NT)-TACC3-depleted mouse oocytes. Green, microtubules (mClover3-MAP4-MTBD); magenta, aMTOCs (CEP192 mScarlet); blue, chromosomes (H2B-miRFP). Time is given as hours:minutes after NEBD. Yellow asterisks highlight the unfocused spindle intermediate. (B and $\mathbf{C}$ ) Quantification of total fluorescence intensity of microtubules and spindle volume in control, TACC3-depleted, and TACC3( $\triangle N$ NT)-TACC3depleted mouse oocytes. The number of analyzed oocytes is specified in italics. Error bars (shaded areas) represent SD. Scale bars, $5 \mu \mathrm{m}$.

anti-centromere antibody (ACA) (FZ90C-CS1058; Europa Bioproducts), rabbit anti-AKAP450 (NBP189167; Novus Biological), rabbit anti-ASPM (NB1002278; Novus Biological), rat anti- $\alpha$-tubulin (MCA78G; Bio-Rad), mouse anti-AURA (NBP2-50041; Novus Biological), rabbit anti-pT288-AURA (NB100-2371; Novus Biological), rabbit anti-CAMSAP3 (48), rabbit anti-CDK5RAP2 (ABE236; Merck Millipore), mouse anti-pan centrin (04-1624; Merck Millipore), rabbit anti-CEP63 (06-1292; Merck Millipore), rabbit anti-CEP120 (PA5-55985; Thermo Fisher Scientific), rabbit anti-CEP135 (ab75005; Abcam), rabbit anti-CEP152 (ab183911; Abcam), rabbit anti-CEP164 (HPA037606; Sigma-Aldrich), rabbit anti-CEP170 (27325-1-AP; Proteintech), rabbit anti-CEP192 (18832-1-AP; Proteintech), mouse antiCHC17 (610500; BD Biosciences), rabbit anti-CHC17 (ab21679; Abcam), rabbit anti-chTOG (PA5-59150 and PA5-58763; Thermo Fisher Scientific), rabbit anti-CNAP1 (14498-1-AP; Proteintech), rabbit antiCP110 (12780-1-AP; Proteintech), rabbit anti-DHC (12345-1-AP; Proteintech), rabbit anti-DISC1 (NB110-40773; Novus Biological), goat anti-GFP (600-101-215; Rockland Immunochemicals), mouse anti-GM130 (610822; BD Biosciences), mouse antiGMAP210 (611712; BD Biosciences), rabbit antiGTSE1 (20), rabbit anti-GTSE1 (A302-425A; Bethyl Laboratories), mouse anti- $\gamma$-tubulin (11-465-C025; Exbio), rabbit anti-HAUS6 (49), rabbit antiHOOK3 (NBP2-44279; Novus Biological), rabbit anti-KANSL3 (HPA035018; Sigma-Aldrich), rabbit
anti-KIF2A(NB500-180; Novus Biologicals), rabbit anti-KIZ (21177-1-AP; Proteintech), mouse anti-LIS1 (H00005048-M03; Abnova), rabbit anti-MCRS1 (HPA039057; Sigma-Aldrich), sheep anti-MCAK (50), rabbit anti-MKLP2 (51), goat anti-MYO10 (sc-23137; Santa Cruz Biotechnology), rabbit antiNDE1 (10233-1-AP; Proteintech), rabbit anti-NDEL1 (H00081565-D01P; Abnova), mouse anti-NEDD1 (H00121441-M05; Abnova), rabbit anit-ninein (ABN1720; Merck Millipore), mouse anti-NUMA (610561; BD Biosciences), rabbit anti-pS395NUMA (3429; Cell Signaling Technology), rabbit anti-NUMA (14951; Cell Signaling Technology), rabbit anti-ODF2 (12058-1-AP; Proteintech), mouse anti-P50 (611002; BD Biosciences), mouse antiP150 (612708; BD Biosciences), rabbit anti-PCM1 (52), rabbit anti-PCM1 (HPA023374; Sigma-Aldrich), mouse anti-pericentrin (611814; BD Biosciences), rabbit anti-pericentrin (ab4448; Abcam), mouse anti-PLK1 (ab17056; Abcam), mouse anti-pT210PLK1 (558400; BD Biosciences), goat anti-PLK4 (NB100-894; Novus Biological), rabbit anti-SSX2IP (HPA027306; Sigma-Aldrich), rabbit anti-STIL (ab89314; Abcam), rabbit anti-SPDL1 (53), goat anti-TACC3 (AF5720-SP; R\&D Systems), mouse anti-TACC3 (H00010460-M02; Abnoa), rabbit antiTACC3 (ab134154; Abcam), rabbit anti-TGN46 (ABT95; Merck Millipore), mouse anti-TOP2A (MAB4197; Merck Millipore), and rabbit anti-TPX2 (NB500-179; Novus Biological). Secondary antibodies used were Alexa Fluor 405-, 488-, 568-, or 647-conjugated anti-human IgG, goat IgG, mouse IgG, mouse IgM, rabbit IgG, rat IgG, or sheep IgG (Molecular Probes). DNA was stained with Hoechst 33342 (Molecular Probes).

\section{Optical clearing of bovine, ovine, and porcine oocytes}

Oocytes were fixed, extracted, and blocked as for routine immunofluorescence. Before incubation with primary antibodies, lipid droplets in oocytes were cleared with $4000 \mathrm{U} / \mathrm{ml}$ lipase from Candida rugose (Sigma-Aldrich) in $400 \mathrm{mM} \mathrm{NaCl}, 50 \mathrm{mM}$ tris ( $\mathrm{pH} \mathrm{7.2),} 5 \mathrm{mM} \mathrm{CaCl}_{2}$, and $0.2 \%$ sodium taurocholate supplemented with complete, EDTA-free Protease Inhibitor Cocktail (Roche) at room temperature for 20 to $40 \mathrm{~min}$.

\section{Protein-retention expansion microscopy}

Expansion microscopy was performed as previously described for cell and tissue samples (54) using the Expansion Microscopy Kit (Expansion Technologies).

\section{Expression constructs, mRNA synthesis, protein expression, and purification}

To generate constructs for mRNA synthesis, we fused previously published coding sequences with meGFP (Clonetech), mClover3 or $3 \times$ mClover3 (55), mPA-GFP (56), $3 \times$ CyOFP (57), mCherry (58), mScarlet (59), or miRFP (60) and subcloned them into pGEMHE (61) to obtain meGFPAKAP450 (62), AURA-meGFP (63), Bbs4-meGFP (64), meGFP-BBS14 (65), TurboGFP-BUGZ (66), mCherry-CDK5RAP2 (67), CEP72-meGFP (68), mScarlet-CEP192 (69), CETN2-meGFP (70), meGFPcentrobin (71), mClover3-CHC17 (21), chTOGmScarlet (72), mEmerald-CLIP170 (M. Davidson, Florida State University, FL), meGFP-CPAP (73), mClover3-GTSE1 (72), H2B-miRFP (4), meGFP-HOOK3 (74), meGFP-KIF2B (75), LRRC36-meGFP (68), LRRC45-meGFP (76), mClover3-MAP4-MTBD (77), $3 \times$ CyOFP-MAP4-MTBD (77), NEDD1-mCherry (78), meGFP-NEK2A (79), meGFP-P50 (80), meGFPP150 (81), meGFP-PAR6 $\alpha$ (82), PCM1-mClover3 (Source BioScience), PCM1-mPA-GFP (Source Bioscience), meGFP-Pericentrin (83), PRC1$3 \times$ mClover3 (72), meGFP-rootletin (84), meGFPSAS6 (85), TACC3-mClover3 (72), TACC3-mPA-GFP (72), mPA-GFP-TPX2 (86), (bovine) TRIM21 (Thermo Fisher Scientific), (mouse) TRIM21 (87), and $\gamma$-tubulin-meGFP (88). EB3-3 $\times$ mCherry was subcloned from pEB3-mCherry (J. Ellenberg, EMBL, Heidelberg, Germany) into pGEMHE. pGEMHE$\operatorname{TACC} 3(\Delta \mathrm{CID})$-mClover3, pGEMHE-TACC3( $\triangle \mathrm{TACC}$ )mClover3, and pGEMHE-TACC3 $(\triangle \mathrm{NT})$ were constructed from pGEMHE-TACC3-mClover3 using QuikChange Lightning Multi Site-Directed Mutagenesis Kit (Agilent) or In-Fusion HD Cloning Plus (Clonetech) or Q5 Site-Directed Mutagenesis Kit (NEB). pGEMHE-mCherry-MAP4-MTBD (77), pGEMHE-mPA- $\alpha$-tubulin (77), pGEMHE-CEP250meGFP (89) and pGEMHE-mCherry-PLK1 (89) were also use. All mRNAs were synthesized and quantified as previously described (90).

To generate constructs for protein expression, TACC3( $\triangle \mathrm{TACC})(\triangle \mathrm{aa} 594-838)$-His6 and TACC (aa594-838)-His were inserted into pGEX-6p-1 
A

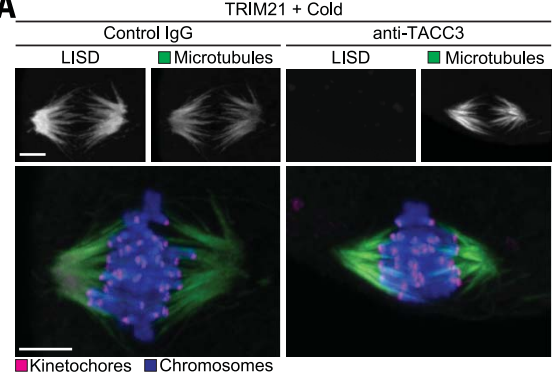

D

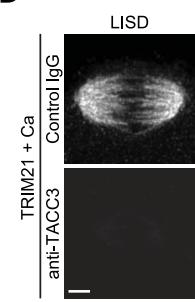

G

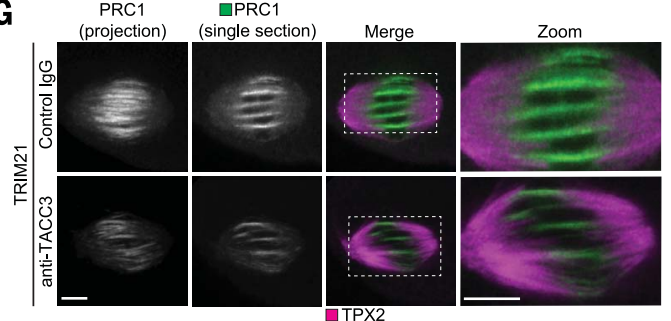

B

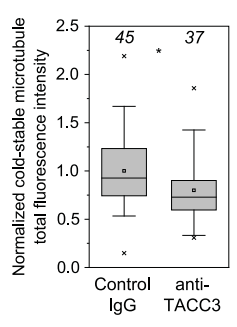

E F

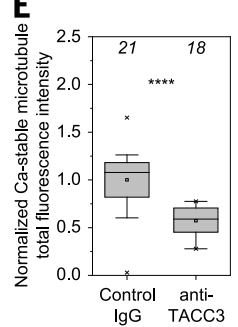

H
C

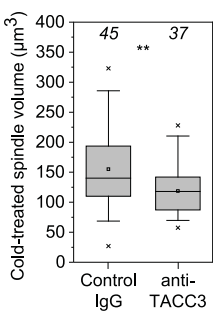

$\mathbf{F}$

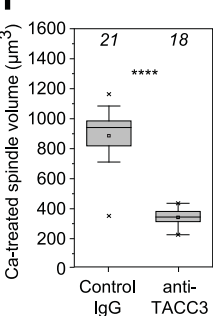

IgG TACC3

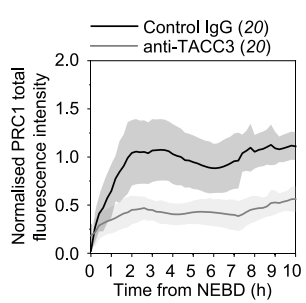

Fig. 7. Loss of K-fibers and interpolar microtubules in TACC3-depleted oocytes. (A) Immunofluorescence images of the metaphase I spindle in cold-treated control and TACC3-depleted mouse oocytes. Gray, LISD (TACC3). Green, microtubules ( $\alpha$-tubulin); magenta, kinetochores (ACA); blue, chromosomes (Hoechst). (B and C) Quantification of total fluorescence intensity of cold-stable microtubules and spindle volume in cold-treated control and TACC3-depleted mouse metaphase I oocytes. (D) Immunofluorescence images of the metaphase I spindle in calcium-treated control and TACC3-depleted mouse oocytes. Green arrowheads highlight stable interpolar microtubules; magenta arrowheads indicate K-fibers. (E and $\mathbf{F}$ ) Quantification of total fluorescence intensity of calcium-stable microtubules and spindle volume in calcium-treated control and TACC3-depleted mouse metaphase I oocytes. (G) Immunofluorescence images of PRC1-3×mClover3 in control and TACC3-depleted mouse metaphase I oocytes. Green, PRC1 (GFP); magenta, TPX2.

(H) Quantification of total fluorescence intensity of PRC1-marked microtubules in control and TACC3-depleted mouse oocytes. The number of analyzed oocytes is specified in italics. Error bars (shaded areas) represent SD. Scale bars, $5 \mu \mathrm{m}$.

(GE Healthcare), and His6-TACC3-His6 was inserted into pET-28c(+) (Novagen). GST-TACC3His6, GST-TACC3( $\triangle$ TACC)-His6, GST-TACC-His6 were expressed in and purified from BL21(DE3) pLysS Competent Cells (Promega) as previously described (26) with some modifications. Briefly, recombinant proteins were purified first using Ni-NTA Agarose (Qiagen), followed by Q Sepharose Fast Flow (GE Healthcare). His6-TACC3-His was expressed in NiCo21(DE3) (NEB) and purified first using Ni-NTA Agarose, followed by Chitin Resin (NEB).

\section{Short-interfering RNAs}

All short interfering RNAs (siRNAs) were purchased from Qiagen. For knockdown of TACC3 using RNAi, a mix of the following siRNAs were used: 5'-AAGTCCTAACATGACCAATAA-3', 5'-CTGCATGTCTTAAATGACGAA-3', 5'-AAGAC-
TAAAGTTTAATCTCAA-3', and 5'-AAGGAAATAGCTGAAGACAAA-3'. AllStars Negative Control (Qiagen) was used as a control.

\section{Microinjection of mouse oocytes, follicles, and bovine oocytes}

Mouse oocytes were microinjected with $3.5 \mathrm{pl}$ of mRNAs as previously described (4). mClover3MAP4-MTBD mRNA was microinjected at a needle concentration of $83.5 \mathrm{ng} / \mu \mathrm{l}, \mathrm{PCM1}-\mathrm{mPA}$ GFP mRNA at $165.8 \mathrm{ng} / \mu \mathrm{l}, \mathrm{PRC1}-3 \times$ mClover3 mRNA at $97 \mathrm{ng} / \mu \mathrm{l}$, TACC3-mClover3 mRNA at $208.1 \mathrm{ng} / \mu \mathrm{l}, \mathrm{TACC} 3-\mathrm{mPA}-\mathrm{GFP}$ at $277.4 \mathrm{ng} / \mu \mathrm{l}, \mathrm{mPA}-$ GFP- $\alpha$-tubulin mRNA at $250.5 \mathrm{ng} / \mu \mathrm{l}, 3 \times$ CyOFPMAP4-MTBD mRNA at $166.4 \mathrm{ng} / \mu \mathrm{l}$, CEP192-mScarlet mRNA at $165.8 \mathrm{ng} / \mu \mathrm{l}, \mathrm{EB} 3-3 \times$ mCherry mRNA at $138.8 \mathrm{ng} / \mu \mathrm{l}, \mathrm{H} 2 \mathrm{~B}-\mathrm{miRFP}$ mRNA at $28.4 \mathrm{ng} / \mu \mathrm{l}$, and Trim21 mRNA at $421 \mathrm{ng} / \mu \mathrm{l}$. For other mRNAs, they were individually titrated and care was taken that they did not induce phenotypes due to overexpression. Oocytes were allowed to express mRNAs for 3 to 4 hours before released into dbcAMP-free medium for imaging.

Mouse follicles were microinjected with $6 \mathrm{pl}$ of siRNAs at a needle concentration of $2 \mu \mathrm{M}$ as previously described (47).

Bovine oocytes were microinjected with $7 \mathrm{pl}$ of TACC3-mClover3 mRNA and bovine TRIM21 mRNA at a needle concentration of 208.1 and $421 \mathrm{ng} / \mu \mathrm{l}$, respectively. Oocytes were allowed to express mRNAs for 3 to 9 hours before released into dbcAMP-free, bicarbonate-buffered BO-IVM (IVF Bioscience).

\section{Peptide preincubation assay}

Oocytes were fixed, extracted, and blocked as for routine immunofluorescence. Rabbit polyclonal anti-CHC17, polyclonal anti-GTSE1, and monoclonal anti-TACC3 were preincubated with peptide PQAPFGYGYTAPPYGQPQPGFGYSM (GenScript), recombinant GTSE1 (OriGene), and recombinant GST-TACC3-His6 (homemade), respectively, before applied to oocytes as previously described (90).

\section{Trim-Away in mouse and bovine oocytes}

Only affinity-purified antibodies were used in this study for Trim-Away-mediated protein depletion. Rabbit polyclonal anti-CHC17, polyclonal anti-GTSE1, and monoclonal anti-TACC3 were purified as previously described (90). The control IgG used was normal rabbit IgG (12-370; Millipore).

For constitutive Trim-Away in mouse GV oocytes, $3.5 \mathrm{pl}$ of mRNAs and $3.5 \mathrm{pl}$ of antibodies were microinjected as previously described (90). All antibodies were microinjected at a needle concentration of $1 \mathrm{mg} / \mathrm{ml}$ with $0.1 \% \mathrm{NP}-40$. Target proteins were allowed to be depleted for 3 to 4 hours before the oocytes were released into dbcAMP-free medium for imaging. For acute Trim-Away in mouse metaphase I oocytes, oocytes were first microinjected with $3.5 \mathrm{pl}$ of mRNAs and then released into dbcAMP-free medium after 3 hours. At $\sim 6$ hours upon release, oocytes were further microinjected with $3.5 \mathrm{pl}$ of antibodies immediately before imaging.

For constitutive Trim-Away in bovine GV oocytes, $7 \mathrm{pl}$ of bovine TRIM21 mRNA and $7 \mathrm{pl}$ of anti-TACC 3 at a needle concentration of $2 \mathrm{mg} / \mathrm{ml}$ with $0.1 \%$ NP-40 were microinjected. TACC3 was allowed to be depleted for 3 to 9 hours before the oocytes were released into dbcAMPfree, bicarbonate-buffered BO-IVM.

\section{Immunoblotting}

Thirty mouse metaphase I oocytes per lane were washed in BSA-free M2 medium and resuspended in $4 \mu$ l of BSA-free M2 medium. $12 \mu$ of $1.333 \times$ NuPAGE LDS sample buffer (Thermo Fisher Scientific) with $100 \mathrm{mM}$ DTT was then added, and the mixture was immediately snap-frozen in liquid nitrogen for $5 \mathrm{~min}$. Samples were thawed and frozen twice more before heated at $100^{\circ} \mathrm{C}$ for $5 \mathrm{~min}$. Samples were resolved on a 15 -well NuPAGE 4 to $12 \%$ bis-tris protein gel of $1.0 \mathrm{~mm}$ thickness (Thermo Fisher Scientific) with NuPAGE 
MOPS SDS Running Buffer (Thermo Fisher Scientific). Proteins were transferred onto a 0.45- $\mu \mathrm{m}$ PVDF membrane with SDS-free Towbin buffer at $200 \mathrm{~mA}$ for 2 hours on ice. Blocking and antibody incubations were performed in trisbuffered saline (TBS) with 5\% skim milk and $0.1 \%$ tween-20. Primary antibodies used were mouse anti-AURA (610938; BD Biosciences), rabbit anti-chTOG, mouse anti-CHC17 rabbit anti-TACC3, and rat- $\alpha$-tubulin. Secondary antibodies used were HRP-conjugated anti-mouse (P0447; Dako), anti-rabbit (31462; Invitrogen), and anti-rat (sc-2032; Santa Cruz Biotechnology). Blots were developed with SuperSignal West Femto Maximum Sensitivity Substrate (Thermo Fisher Scientific) and documented with Amersham Imager 600 (GE Healthcare). Care was taken that the exposure time did not cause saturation.

\section{Confocal and super-resolution microscopy}

For confocal imaging, oocytes were imaged in 1 to $2 \mu$ l of M2 medium (for live mouse oocytes) or PBS with $1 \%$ polyvinylpyrrolidone (PVP) and $0.5 \mathrm{mg} / \mathrm{ml} \mathrm{BSA}$ (for fixed oocytes) under paraffin oil in a $35-\mathrm{mm}$ dish with a \#1.0 coverslip. Images were acquired with LSM 880 confocal laser scanning microscopes (Zeiss) equipped with an environmental incubator box and a 40x C-Apochromat 1.2 NA water-immersion objective. A volume of $50 \mu \mathrm{m}$ by $50 \mu \mathrm{m}$ by $37.5 \mu \mathrm{m}$ or $35 \mu \mathrm{m}$ by $35 \mu \mathrm{m}$ by $37.5 \mu \mathrm{m}$ centered around the chromosomes was typically recorded. Automatic $3 \mathrm{D}$ tracking was implemented for time-lapse imaging with a temporal resolution of 5 to $15 \mathrm{~min}$ using Autofocuscreen (91) or MyPiC (92). mClover3 and meGFP were excited with a 488-nm laser line and detected at 493 to $571 \mathrm{~nm}$. CyOFP was excited with a 488-nm laser line and detected at 571 to $638 \mathrm{~nm}$. mScarlet and mCherry were excited with a $561-\mathrm{nm}$ laser line and detected at 571 to $638 \mathrm{~nm}$. miRFP was excited with a $633-\mathrm{nm}$ laser line and detected at 638 to $700 \mathrm{~nm}$. Images of the control and experimental groups were acquired under identical imaging conditions on the same microscope. For some images, shot noise was reduced with a Gaussian filter. Airyscan images were acquired using the Airyscan module on LSM800 and LSM880 confocal laser scanning microscopes (Zeiss) and processed in ZEN (Zeiss) after acquisition. Care was taken that the imaging conditions (laser power, pixel-dwell time, and detector gain) did not cause phototoxicity (for live imaging), photobleaching, or saturation.

\section{Drug addition and washout}

All drugs except 1,6-hexanediol were prepared in DMSO (Sigma-Aldrich) as 1000× stocks. To depolymerize microtubules, metaphase I oocytes were treated with $10 \mu \mathrm{M}$ nocodazole (SigmaAldrich). To regrow microtubules, metaphase I oocytes were first washed into $10 \mu \mathrm{M}$ nocodazole for 45 to $60 \mathrm{~min}$ and then washed out into medium without drug. To inhibit EG5, metaphase I oocytes were treated with $100 \mu \mathrm{M}$ monastrol (Sigma-Aldrich) for 30 to $90 \mathrm{~min}$. To inhibit AURA, PLK1, and PLK4 in metaphase I oocytes, MLN8237 (Selleckchem), BI2536 (Selleckchem) and centrinone were used at $500 \mathrm{nM}, 100 \mathrm{nM}$, and $5 \mu \mathrm{M}$, respectively. Previous studies have shown that these drugs do not exhibit nonspecific inhibition on other kinases at the indicated concentrations $(5,89,93)$. 1,6-hexanediol (Sigma-Aldrich) was prepared as a $10 \times$ stock in medium and used at a final concentration of $3.5 \%$.

\section{Electron microscopy staining, immunoelectron microscopy, and focused ion beam-scanning electron microscopy}

For electron microscopy staining, mouse metaphase I oocytes were fixed as previously described for mitotic cells (94) with some minor modifications. Briefly, oocytes were fixed in 100 mM HEPES (pH 7.0, titrated with $\mathrm{KOH}$ ), $50 \mathrm{mM}$ EGTA (pH 7.0, titrated with KOH), $10 \mathrm{mM}$ $\mathrm{MgSO}_{4}, 3 \%$ sucrose, $3 \%$ EM-grade glutaraldehyde, $0.5 \%$ methanol-free formaldehyde, and $0.1 \%$ tannic acid at $37^{\circ} \mathrm{C}$ for 1 hour. Fixed oocytes were first stained with $2 \%$ osmium tetroxide (Electron Microscopy Sciences)-1.5\% potassium ferrocyanide (Electron Microscopy Sciences) in $0.1 \mathrm{M}$ phosphate buffer ( $\mathrm{pH}$ 7.4) for 1 hour. Oocytes were then incubated with $0.1 \%$ tannic acid (Sigma-Aldrich) for $30 \mathrm{~min}$ and stained with $2 \%$ osmium tetroxide in water for $40 \mathrm{~min}$. After overnight staining with $1 \%$ uranyl acetate (SPIChem) in water at $4^{\circ} \mathrm{C}$, oocytes were further stained with $0.02 \mathrm{M}$ lead nitrate (Merck Millipore)-0.03 M aspartic acid (Sigma-Aldrich) (pH 5.5) for $30 \mathrm{~min}$. Oocytes were washed three times with water for 5 min between every staining step.

For immunoelectron microscopy of TACC3, mouse metaphase I oocytes were microinjected with $7 \mathrm{pl}$ of $0.3 \mathrm{mg} / \mathrm{ml}$ US Immunogold-conjugated rabbit Fab anti-TACC3 (Aurion) with 0.1\% NP-40. After fixation as described above, silver enhancement was performed with R-Gent SE-EM (Aurion). All following processing steps were performed in a microwave (Ted Pella) and oocytes were washed three times with water for $40 \mathrm{~s}$ at $250 \mathrm{~W}$ between every staining step. Fixed oocytes were first stained with $2 \%$ osmium tetroxide- $1.5 \%$ potassium ferrocyanide in $0.1 \mathrm{M}$ phosphate buffer ( $\mathrm{pH}$ 7.4) for $8 \mathrm{~min}$ at $100 \mathrm{~W}$ (microwave cycling between on and off every $2 \mathrm{~min}$ ). Oocytes were then incubated with $1 \%$ thiocarbohydrazide (Sigma-Aldrich) for $12 \mathrm{~min}$ at $100 \mathrm{~W}$ (microwave cycling between on and off every $2 \mathrm{~min}$ ) and stained with $2 \%$ osmium tetroxide in water for $12 \mathrm{~min}$ at $100 \mathrm{~W}$ (microwave cycling between on and off every $2 \mathrm{~min}$ ). After overnight staining with $1 \%$ uranyl acetate in water at $4{ }^{\circ} \mathrm{C}$, oocytes were further stained with $0.02 \mathrm{M}$ lead nitrate$0.03 \mathrm{M}$ aspartic acid (pH 5.5) for $12 \mathrm{~min}$ at $100 \mathrm{~W}$ (microwave cycling between on and off every 2 min).

Oocytes were subsequently dehydrated in a graded ethanol series $(10,30,50,75,90,100$, and $100 \%$ ) for $40 \mathrm{~s}$ at $250 \mathrm{~W}$ and infiltrated in a graded series $(25,50,75,90,100$, and $100 \%)$ of Durcupan resin (Sigma-Aldrich) in ethanol for $3 \mathrm{~min}$ at $250 \mathrm{~W}$. Infiltrated oocytes were embedded with minimal amount of resin on top of an Aclar film as previously described (95). Embedded oocytes were cut out and attached to a SEM stub (Science Services) using silver-filled EPO-TEK EE129-4 adhesive (Electron Microscopy Sciences), and were cured overnight at $60^{\circ} \mathrm{C}$. Samples were coated with an 8-nm platinum layer using the high-vacuum sputter coater EM ACE600 (Leica) at 35-mA current. Afterwards, samples were placed in the Crossbeam 540 FIBSEM (Zeiss). To ensure even milling and to protect the surface, a $400-\mathrm{nm}$ platinum layer was deposited on top of the region of interest at 3-nA current. Atlas 3D (Fibics) was used to collect the $3 \mathrm{D}$ datasets. Samples were exposed with a 15-nA current, and a 7-nA current was used to polish the cross-section surface. Images were acquired at $1.5 \mathrm{kV}$ with the ESB detector at a grid voltage of $1100 \mathrm{~V}$ (5-nm pixel size in $x y$ ) in a continuous mill and acquire mode using $700 \mathrm{pA}$ for the milling aperture (5-nm $z$-step).

After acquisition, images were first aligned using Linear Stack Alignment with SIFT in Fiji (NIH) (96). Datasets were then cropped, inverted and smoothed in Fiji. For better visualization of microtubules, datasets were further subjected to two rounds of Local contrast enhancement (CLAHE) in Fiji. Specific parameters used for the first round were: 127 for blocksize, 100 for histogram bins, and 1.5 for maximum slope. Specific parameters used for the second round were 50 for blocksize, 256 for histogram bins, and 2.0 for maximum slope. To obtain a metaphase I spindle parallel to the imaging plane, the resulting image stacks were rotated and resliced with Interactive Stack Rotation in Fiji. 3D segmentation and surface rendering were performed with Microscopy Image Browser (97) and Imaris, respectively.

\section{Fluorescence recovery after photobleaching (FRAP)}

For analyses of protein dynamics, oocytes coexpressing fluorescent reporter(s) of interest with H2B-miRFP were rotated with an unbroken microinjection needle on stage to obtain meiotic spindles parallel to the imaging plane. Rectangular or circular regions of interest (ROIs) were marked and photobleached using the corresponding excitation laser line $(405,488,561$, and/or $633 \mathrm{~nm}$ ) at the maximum power after the third or fifth time point.

\section{Photoactivation}

For analyses of dissipation, oocytes coexpressing mPA- $\alpha$-tubulin, PCM1-mPA-GFP, TACC3-mPAGFP, or mPA-GFP-TPX2 with H2B-miRFP were rotated with an unbroken microinjection needle on stage to obtain meiotic spindles parallel to the imaging plane. Rectangular ROIs were marked and photoactivated using the 405 -nm laser line at the maximum power after the third or fifth time point.

\section{In vitro droplet assembly and dextran exclusion assay}

TACC3 droplets were assembled in an imaging chamber as previously described for SPD-5 (32). 
For most experiments, $20 \mu \mathrm{M}$ purified His-tagged proteins or recombinant GST (GenScript) in $50 \mathrm{mM}$ tris (pH 7.4), $500 \mathrm{mM} \mathrm{NaCl}, 0.5 \mathrm{mM}$ DTT, $1 \%$ glycerol, and $0.1 \%$ CHAPS (all from SigmaAldrich) was added to $25 \mathrm{mM}$ HEPES (pH 6.4), $150 \mathrm{mM} \mathrm{KCl}, 0.5 \mathrm{mM}$ DTT, and 12\% PEG-3350 with $0.1 \mathrm{mg} / \mathrm{ml}$ NTA-Atto $647 \mathrm{~N}$ (all from Sigma-Aldrich). For dextran exclusion assay, $2.5 \mathrm{mg} / \mathrm{ml}$ Oregon Green 488-conjugated dextran of 70,000 M.W. (Molecular Probes) was included during the assembly of the reaction.

\section{Thioflavin $T$ staining}

Oocytes were fixed, extracted, and blocked as for routine immunofluorescence. $10 \mu \mathrm{M}$ thioflavin T was applied to oocytes for $10 \mathrm{~min}$ as previously described (98).

\section{Cold-stable assay}

Mouse metaphase I oocytes were incubated on ice for $15 \mathrm{~min}$ and immediately fixed as for routine immunofluorescence.

\section{Calcium-stable assay}

Mouse metaphase I oocytes were incubated in $100 \mathrm{mM}$ PIPES ( $\mathrm{pH} 7.0$, titrated with $\mathrm{KOH}$ ), $1 \mathrm{mM} \mathrm{MgCl}_{2}, 0.1 \mathrm{mM} \mathrm{CaCl}_{2}$, and $0.1 \%$ triton X-100 at $37^{\circ} \mathrm{C}$ for $15 \mathrm{~min}$ and immediately fixed as for routine immunofluorescence.

\section{General quantification}

Time-lapse movies of live mouse oocytes were analyzed using Imaris (Bitplane). To determine the stages of meiosis and to score for chromosome segregation defects, the timing of meiotic progression was quantified relative to the time of NEBD. NEBD was defined as the time point when the sharp boundary between the nucleus and cytoplasm disappeared. Chromosome(s) that failed to congress to the metaphase plate before anaphase onset or the end of the video were scored as misaligned chromosomes. Anaphase onset was defined as the last time point of metaphase ( 5 to $6 \mathrm{~min}$ ) before chromosome separation was first observed. Chromosome(s) that failed to clear the central spindle region within 10 to 12 and 20 to 24 min of anaphase onset were scored as mildly and severely lagging chromosomes, respectively. The presence of misaligned or lagging chromosome(s) was further confirmed by $3 \mathrm{D}$ reconstruction of chromosomes in Imaris as previously described (77).

\section{Quantification of FRAP experiments}

Mean intensities of photobleached areas over time were exported from ZEN into Excel (Microsoft) for further processing. The intensity of an unbleached area in the cytoplasm of the same oocyte was subtracted to correct for cytoplasmic background. Background-corrected data were then normalized to the mean intensity of prebleach time points $\left(F_{0}\right)$. As the postbleach intensity could not be normalized between individual plots due to variable photobleaching efficiency, plots of intensity $(F)$ against time were fitted to single exponential functions $\left[F(t)=c-F_{\infty} \mathrm{e}^{-t / \tau}\right.$ where $c$ is the offset, $F_{\mathrm{\infty}}$ is the amplitude of maximum intensity recov- ered after equilibrium, and $\tau$ is the time constant) in OriginPro (OriginLab). Half-times of maximum recovery $\left(t_{1 / 2}\right)$ and mobile fractions were determined by $\tau \times \ln (2)$ and $F_{\infty} /\left(F_{0}-F^{\prime}\right)$ (where $F^{\prime}$ is the minimum intensity measured immediately after photobleaching), respectively.

\section{Manual and automatic quantification of plus-end growth velocity}

To determine the growth rate of plus-ends of astral microtubules, comet velocities were determined using the Kymograph function in Fiji. Tracks were analyzed in Excel.

For plus-ends of microtubules within the spindle, comets were automatically tracked using u-track 2.2 (99) in MATLAB R2017b (MathWorks). For comet detection, low-pass and high-pass Gaussian standard deviations were set to two and six pixels, respectively. For comet tracking, maximum gap to close was set to 0 frame to eliminate discontinuous tracks. As astral microtubules were protruding from the spindle in all directions and their comets should be out of focus before comets of spindle microtubules, tracks longer than five frames were used for further processing. This resulted in, on average, $>500$ (for control group) and $>300$ (for experimental group) tracks per oocyte within $2.5 \mathrm{~min}$.

\section{Quantification of} photoactivation experiments

To determine dissipation rates of $\alpha$-tubulin, mean intensities of photoactivated areas over time were exported from ZEN into Excel for further processing. Data were first corrected for cytoplasmic background by subtracting the intensity of the photoactivated areas before photoactivation. Background-corrected data were then normalized to the intensity of the first postactivation time point $\left(F_{0}\right)$. Plots of intensity $(F)$ against time were fitted to one-component exponential functions $\left[y=A \mathrm{e}^{(c-x) / \tau}\right.$, where $c$ is the offset, $A$ is the fraction of the component, and $\tau$ is the time constant] in OriginPro. Half-times of decay $\left(t_{1 / 2}\right)$ were calculated by $\tau \times \ln (2)$. Singlecomponent exponential fitting was used because double-component exponential fitting was unable to yield two distinct $\tau$, consistent with our previous studies on metaphase II spindles (77). This was likely due to the fact that the fraction of dynamic non-kinetochore-bound microtubules is much more abundant than that of stable kinetochore microtubules in mouse meiotic spindles.

\section{Manual quantification of fluorescence intensities and volume by 3D reconstruction of spindles and aMTOCs}

Spindles and aMTOCs were labeled (with MAP4MTBD and CEP192, respectively) in live and (with $\alpha$-tubulin and pericentrin, respectively) fixed oocytes. They were segmented by applying a threshold in the corresponding channel using the Surface function of Imaris. Cytoplasmic aMTOCs that were not part of the spindle were manually removed. Depending on the signal-tonoise ratio in each oocyte, a suitable threshold value was selected and maintained for the entire time series. The volumes of spindles and aMTOCs were exported into Excel. The mean and total intensities for spindle proteins ( $\alpha$-tubulin, MAP4MTBD, PRC1, or TACC3) and aMTOCs proteins (CEP192 or Pericentrin) were exported into Excel.

For live imaging, data from individual oocytes were aligned to the time of NEBD or the onset of microtubule nucleation. To normalize individual dataset, all (mean or sum) intensities for a channel from both the control and experimental groups were divided by the average (mean or total) intensity for that channel from either the control or experimental group at steady state. For some experiments, normalized data from different datasets were interpolated to identical time intervals in OriginPro.

For immunofluorescence, all (mean or sum) intensities for a channel from both the control and experimental groups were normalized to the average (mean or total) intensity for that channel from the control group.

\section{Automatic quantification of fluorescence intensities and volume by 3D reconstruction of spindles}

To consistently reconstruct the spindles from different cold-stable assays, an in-house-developed MATLAB script (https://github.com/Emoenni/ batch_surfaces) was used for automatic surface creation in Imaris.

Individual sections of a dataset were backgroundsubtracted by a Gaussian smoothed image with a sigma equal to $80 \%$ of the background scale given and smoothed with a median filter. The size of the filter was determined by the nearest odd integer to twice the object detail given.

To automatically determine a threshold for the Surface function in Imaris, Otsu's method was implemented via the "multithresh.m" function in MATLAB. This function allows the computation of multiple thresholds for multilevel images. A single threshold was first computed and the volume of the surface was approximated by multiplying the number of voxels above the threshold with the volume of a voxel. When the volume was not within the range of possible volumes, more thresholds were computed. This was repeated until an appropriate threshold was found or a number of five thresholds was reached. The unaltered values of object detail, background scale, and a filter based on the intensity mean of the second channel were then used for surface creation in Imaris. For spindles, the common center of homogeneous mass of all surfaces was computed, and all surfaces with a center of mass $>10 \mu \mathrm{m}$ from the common one were removed.

Specific parameters used for spindles were: $0.15 \mu \mathrm{m}$ for object detail, $0.6 \mu \mathrm{m}$ for background scale, and 50 to $1000 \mu \mathrm{m}^{3}$ for volume range.

\section{Statistical analysis}

Average (mean) and standard deviation (SD) were calculated in Excel. Statistical significance based on unpaired, two-tailed Student's $t$ test (for absolute values) and two-tailed Fisher's exact test (for categorical values) were calculated in OriginPro or Prism (GraphPad). All box plots show median 
(horizontal black line), mean (small black squares), 25th and 75th percentiles (boxes), 5th and 95th percentiles (whiskers), and 1st and 99th percentiles (crosses). All data are from at least two independent experiments. $P$ values are designated as ${ }^{*} P<0.05,{ }^{* *} P<0.01,{ }^{* * *} P<0.001$, and ${ }^{* * * *} P<0.0001$. Nonsignificant values are indicated as NS.

\section{REFERENCES AND NOTES}

1. D. Szollosi, P. Calarco, R. P. Donahue, Absence of centrioles in the first and second meiotic spindles of mouse oocytes. J. Cell Sci. 11, 521-541 (1972). pmid: 5076360

2. A. M. Meireles, K. H. Fisher, N. Colombié, J. G. Wakefield, H. Ohkura, Wac: A new Augmin subunit required for chromosome alignment but not for acentrosomal microtubule assembly in female meiosis. J. Cell Biol. 184, 777-784 (2009). doi: 10.1083/jcb.200811102; pmid: 19289792

3. N. Colombié, A. A. Głuszek, A. M. Meireles, H. Ohkura, Meiosis-specific stable binding of augmin to acentrosomal spindle poles promotes biased microtubule assembly in oocytes. PLOS Genet. 9, e1003562 (2013). doi: 10.1371/ journal.pgen.1003562; pmid: 23785300

4. P. Romé, H. Ohkura, A novel microtubule nucleation pathway for meiotic spindle assembly in oocytes. J. Cell Biol. 217 3431-3445 (2018). doi: 10.1083/jcb.201803172; pmid: 30087124

5. R. F. Reschen et al., Dgp71WD is required for the assembly of the acentrosomal Meiosis I spindle, and is not a general targeting factor for the $\gamma$-TuRC. Biol. Open 1, 422-429 (2012). doi: 10.1242/bio.2012596; pmid: 23213433

6. T. Cavazza, I. Vernos, The RanGTP pathway: From nucleo-cytoplasmic transport to spindle assembly and beyond. Front. Cell Dev. Biol. 3, 82 (2016). doi: 10.3389/ fcell.2015.00082; pmid: 26793706

7. B. Wang, M. J. Pfeiffer, H. C. Drexler, G. Fuellen, M. Boiani, Proteomic analysis of mouse oocytes identifies PRMT7 as a reprogramming factor that replces SOX2 in the induction of pluripotent stem cells. J. Proteome Res. 15, 2407-2421 (2016). doi: 10.1021/acs.jproteome.5b01083; pmid: 27225728

8. G. Manandhar, H. Schatten, P. Sutovsky, Centrosome reduction during gametogenesis and its significance. Biol. Reprod. 72, 2-13 (2005). doi: 10.1095/ biolreprod.104.031245; pmid: 15385423

9. M. Schuh, J. Ellenberg, Self-organization of MTOCs replaces centrosome function during acentrosomal spindle assembly in live mouse oocytes. Cell 130, 484-498 (2007). doi: 10.1016/j.cell.2007.06.025; pmid: 17693257

10. Y. L. Wong et al., Cell biology. Reversible centriole depletion with an inhibitor of Polo-like kinase 4. Science $\mathbf{3 4 8}$ 1155-1160 (2015). doi: 10.1126/science.aaa5111: pmid: 25931445

11. L. Bury et al., PIk4 and Aurora A cooperate in the initiation of acentriolar spindle assembly in mammalian oocytes. J. Cell Biol. 216, 3571-3590 (2017). doi: 10.1083/jcb.201606077; pmid: 28972102

12. A. N. Kettenbach et al., Quantitative phosphoproteomics identifies substrates and functional modules of Aurora and Polo-like kinase activities in mitotic cells. Sci. Signal. 4 rs5 (2011). doi: 10.1126/scisignal.2001497; pmid: 21712546

13. T. P. Barros, K. Kinoshita, A. A. Hyman, J. W. Raff, Aurora A activates D-TACC-Msps complexes exclusively at centrosomes to stabilize centrosomal microtubules. J. Cell Biol. 170, 1039-1046 (2005). doi: 10.1083/ jcb.200504097; pmid: 16186253

14. K. Kinoshita et al., Aurora A phosphorylation of TACC3/ maskin is required for centrosome-dependent microtubule assembly in mitosis. J. Cell Biol. 170, 1047-1055 (2005). doi: 10.1083/jcb.200503023; pmid: 16172205

15. I. Peset et al., Function and regulation of Maskin, a TACC family protein, in microtubule growth during mitosis. J. Cell Biol. 170, 1057-1066 (2005). doi: 10.1083/jcb.200504037; pmid: 16172207

16. K. Jiang et al., Microtubule minus-end stabilization by polymerization-driven CAMSAP deposition. Dev. Cell 28, 295-309 (2014). doi: 10.1016/j.devcel.2014.01.001; pmid: 24486153

17. F. Gergely, V. M. Draviam, J. W. Raff, The ch-TOG/XMAP215 protein is essential for spindle pole organization in human somatic cells. Genes Dev. 17, 336-341 (2003). doi: 10.1101/ gad.245603; pmid: 12569123

18. G. J. Brouhard et al., XMAP215 is a processive microtubule polymerase. Cell 132, 79-88 (2008). doi: 10.1016/ j.cell.2007.11.043; pmid: 18191222

19. S. Bendre et al., GTSE1 tunes microtubule stability for chromosome alignment and segregation by inhibiting the microtubule depolymerase MCAK. J. Cell Biol. 215, 631-647 (2016). doi: 10.1083/jcb.201606081; pmid: 27881713

20. A. R. Tipton, J. D. Wren, J. R. Daum, J. C. Siefert, G. J. Gorbsky, GTSE1 regulates spindle microtubule dynamics to control Aurora B kinase and Kif4A chromokinesin on chromosome arms. J. Cell Biol. 216, 3117-3132 (2017). doi: 10.1083/jcb.201610012; pmid: 28821562

21. D. G. Booth, F. E. Hood, I. A. Prior, S. J. Royle, A TACC3/ ch-TOG/clathrin complex stabilises kinetochore fibres by inter-microtubule bridging. EMBO J. 30, 906-919 (2011). doi: 10.1038/emboj.2011.15; pmid: 21297582

22. H. Zhu, J. A. Coppinger, C. Y. Jang, J. R. Yates 3rd, G. Fang, FAM29A promotes microtubule amplification via recruitment of the NEDD1-gamma-tubulin complex to the mitotic spindle. J. Cell Biol. 183, 835-848 (2008). doi: 10.1083/ jcb.200807046; pmid: 19029337

23. S. Lawo et al., HAUS, the 8-subunit human Augmin complex, regulates centrosome and spindle integrity. Curr. Biol. 19 816-826 (2009). doi: 10.1016/j.cub.2009.04.033; pmid: 19427217

24. S. Meunier et al., An epigenetic regulator emerges as microtubule minus-end binding and stabilizing factor in mitosis. Nat. Commun. 6, 7889 (2015). doi: 10.1038/ ncomms8889; pmid: 26243146

25. C. H. Lin, C. K. Hu, H. M. Shih, Clathrin heavy chain mediates TACC3 targeting to mitotic spindles to ensure spindle stability. J. Cell Biol. 189, 1097-1105 (2010). doi: 10.1083/ jcb.200911120; pmid: 20566684

26. F. E. Hood et al., Coordination of adjacent domains mediates TACC3-ch-TOG-clathrin assembly and mitotic spindle binding. J. Cell Biol. 202, 463-478 (2013). doi: 10.1083/ jcb.201211127; pmid: 23918938

27. S. F. Banani, H. O. Lee, A. A. Hyman, M. K. Rosen, Biomolecular condensates: Organizers of cellular biochemistry. Nat. Rev. Mol. Cell Biol. 18, 285-298 (2017). doi: 10.1038/nrm.2017.7; pmid: 28225081

28. Y. Shin et al., Spatiotemporal control of intracellular phase transitions using light-activated optoDroplets. Cell 168 159-171.e14 (2017). doi: 10.1016/j.cell.2016.11.054; pmid: 28041848

29. S. Kroschwald, S. Maharana, A. Simon, Hexanediol: A chemical probe to investigate the material properties of membrane-less compartments. Matters (2017) doi: 10.19185/matters.201702000010

30. S. Boeynaems et al., Protein phase separation: A new phase in cell biology. Trends Cell Biol. 28, 420-435 (2018). doi: 10.1016/j.tcb.2018.02.004; pmid: 29602697

31. H. Jiang et al., Phase transition of spindle-associated protein regulate spindle apparatus assembly. Cell 163, 108-122 (2015). doi: 10.1016/j.cell.2015.08.010; pmid: 26388440

32. J. B. Woodruff et al., The centrosome is a selective condensate that nucleates microtubules by concentrating tubulin. Cell 169, 1066-1077.e10 (2017). doi: 10.1016/ j.cell.2017.05.028; pmid: 28575670

33. S. Montenegro Gouveia et al., PLK4 is a microtubuleassociated protein that self-assembles promoting de novo MTOC formation. J. Cell Sci. 132, jcs.219501 (2018). pmid: 30237222

34. M. Mahdipour et al., TACC3 is important for correct progression of meiosis in bovine oocytes. PLOS ONE 10 , e0132591 (2015). doi: 10.1371/journal.pone.0132591; pmid: 26168150

35. W. Fu et al., Self-assembly and sorting of acentrosomal microtubules by TACC3 facilitate kinetochore capture during the mitotic spindle assembly. Proc. Natl. Acad. Sci. U.S.A. 110, 15295-15300 (2013). doi: 10.1073/pnas.1312382110 pmid: 24003142

36. L. Schneider et al., The transforming acidic coiled coil 3 protein is essential for spindle-dependent chromosome alignment and mitotic survival. J. Biol. Chem. 282 , 29273-29283 (2007). doi: 10.1074/jbc.M704151200; pmid: 17675670

37. W. Fu et al., Clathrin recruits phosphorylated TACC3 to spindle poles for bipolar spindle assembly and chromosome alignment. J. Cell Sci. 123, 3645-3651 (2010). doi: 10.1242/ ics.075911; pmid: 20923838
38. L. P. Cheeseman, E. F. Harry, A. D. McAinsh, I. A. Prior, S. J. Royle, Specific removal of TACC3-ch-TOG-clathrin at metaphase deregulates kinetochore fiber tension. J. Cell Sci. 126, 2102-2113 (2013). doi: 10.1242/jcs.124834 pmid: 23532825

39. T. S. Kitajima, M. Ohsugi, J. Ellenberg, Complete kinetochore tracking reveals error-prone homologous chromosome biorientation in mammalian oocytes. Cell 146, 568-581 (2011). doi: 10.1016/j.cell.2011.07.031; pmid: 21854982

40. J. Kajtez et al., Overlap microtubules link sister k-fibres and balance the forces on bi-oriented kinetochores. Nat. Commun. 7, 10298 (2016). doi: 10.1038/ncomms10298; pmid: 26728792

41. H. Maiato, M. Lince-Faria, The perpetual movements of anaphase. Cell. Mol. Life Sci. 67, 2251-2269 (2010) doi: 10.1007/s00018-010-0327-5; pmid: 20306325

42. J. B. Woodruff, Assembly of mitotic structures through phase separation. J. Mol. Biol. 430, 4762-4772 (2018) doi: 10.1016/i.jmb.2018.04.041; pmid: 29751016

43. F. Gergely et al., The TACC domain identifies a family of centrosomal proteins that can interact with microtubules. Proc. Natl. Acad. Sci. U.S.A. 97, 14352-14357 (2000). doi: 10.1073/pnas.97.26.14352; pmid: 11121038

44. M. C. Good, M. D. Vahey, A. Skandarajah, D. A. Fletcher R. Heald, Cytoplasmic volume modulates spindle size during embryogenesis. Science 342, 856-860 (2013). doi: 10.1126/ science.1243147; pmid: 24233724

45. T. Cavazza, P. Malgaretti, I. Vernos, The sequential activation of the mitotic microtubule assembly pathways favors bipolar spindle formation. Mol. Biol. Cell 27, 2935-2945 (2016). doi: 10.1091/mbc.E16-05-0322; pmid: 27489339

46. B. Lacroix et al., Microtubule dynamics scale with cell size to set spindle length and assembly timing. Dev. Cell 45 496-511.e6 (2018). doi: 10.1016/j.devcel.2018.04.022 pmid: 29787710

47. S. Pfender et al., Live imaging RNAi screen reveals genes essential for meiosis in mammalian oocytes. Nature $\mathbf{5 2 4}$, 239-242 (2015). doi: 10.1038/nature14568; pmid: 26147080

48. N. Tanaka, W. Meng, S. Nagae, M. Takeichi, Nezha/CAMSAP3 and CAMSAP2 cooperate in epithelial-specific organization of noncentrosomal microtubules. Proc. Natl. Acad. Sci. U.S.A. 109, 20029-20034 (2012). doi: 10.1073/pnas.1218017109; pmid: 23169647

49. S. Watanabe, G. Shioi, Y. Furuta, G. Goshima, Intra-spindle microtubule assembly regulates clustering of microtubuleorganizing centers during early mouse development. Cell Reports 15, 54-60 (2016). doi: 10.1016/j.celrep.2016.02.087; pmid: 27052165

50. P. D. Andrews et al., Aurora B regulates MCAK at the mitotic centromere. Dev. Cell 6, 253-268 (2004). doi: 10.1016/ S1534-5807(04)00025-5; pmid: 14960279

51. S. Hümmer, T. U. Mayer, Cdk1 negatively regulates midzone localization of the mitotic kinesin Mklp2 and the chromosomal passenger complex. Curr. Biol. 19, 607-612 (2009). doi: 10.1016/j.cub.2009.02.046; pmid: 19303298

52. A. Dammermann, A. Merdes, Assembly of centrosomal proteins and microtubule organization depends on PCM-1. J. Cell Biol. 159, 255-266 (2002). doi: 10.1083/jcb.200204023; pmid: 12403812

53. R. Gassmann et al., Removal of Spindly from microtubuleattached kinetochores controls spindle checkpoint silencing in human cells. Genes Dev. 24, 957-971 (2010). doi: 10.1101/ gad.1886810; pmid: 20439434

54. P. W. Tillberg et al., Protein-retention expansion microscopy of cells and tissues labeled using standard fluorescent proteins and antibodies. Nat. Biotechnol. 34, 987-992 (2016). doi: 10.1038/nbt.3625; pmid: 27376584

55. B. T. Bajar et al., Improving brightness and photostability of green and red fluorescent proteins for live cell imaging and FRET reporting. Sci. Rep. 6, 20889 (2016). doi: 10.1038/ srep20889; pmid: 26879144

56. G. H. Patterson, J. Lippincott-Schwartz, A photoactivatable GFP for selective photolabeling of proteins and cells. Science 297, 1873-1877 (2002). doi: 10.1126/science.1074952; pmid: 12228718

57. J. Chu et al., A bright cyan-excitable orange fluorescent protein facilitates dual-emission microscopy and enhances bioluminescence imaging in vivo. Nat. Biotechnol. 34 760-767 (2016). doi: 10.1038/nbt.3550; pmid: 27240196

58. N. C. Shaner et al., Improved monomeric red, orange and yellow fluorescent proteins derived from Discosoma sp. red fluorescent protein. Nat. Biotechnol. 22, 1567-1572 (2004). doi: 10.1038/nbt1037; pmid: 1555804 


\section{Science $\triangle$ IAAAS}

\section{Supplementary Materials for}

\section{A liquid-like spindle domain promotes acentrosomal spindle assembly in mammalian oocytes}

Chun So ${ }^{\dagger}$, K. Bianka Seres ${ }^{\dagger}$, Anna M. Steyer, Eike Mönnich, Dean Clift, Anastasija Pejkovska, Wiebke Möbius, Melina Schuh*

†These authors contributed equally to this work.

*Correspondence to: melina.schuh@mpibpc.mpg.de

This PDF file includes:

Figs. S1 to S26

Tables S1

References (102-118)

Captions for Movies S1 to S10

Other Supplementary Materials for this manuscript include the following:

Movies S1 to S10 
Figure S1

A

Proteins localizing to the LISD
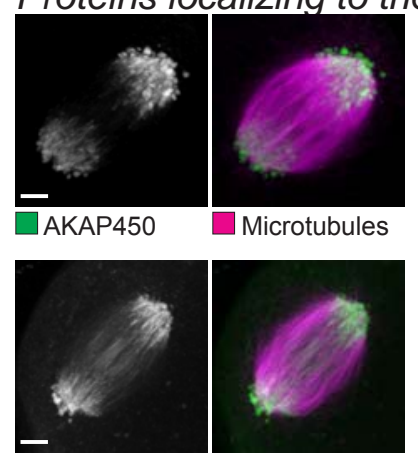

- $\mathrm{CHC} 17$

Microtubules
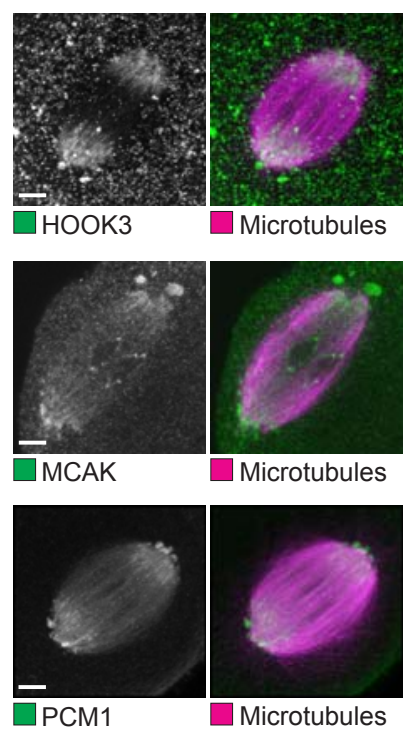
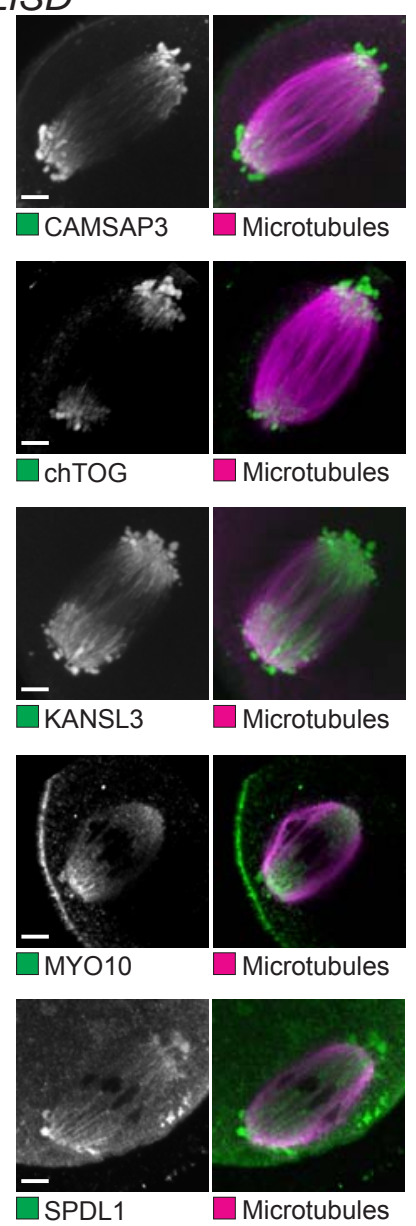

- $\mathrm{SPDL} 1$

\section{-}
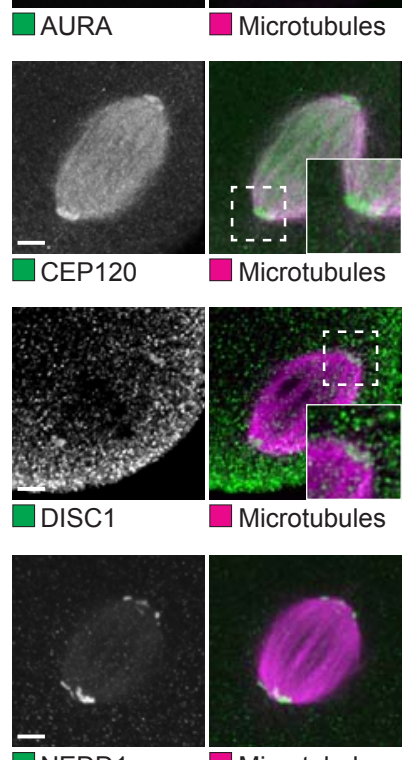

\section{$\square$ NEDD1}
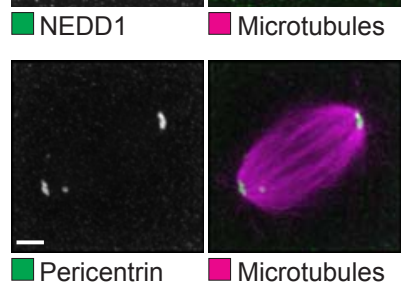
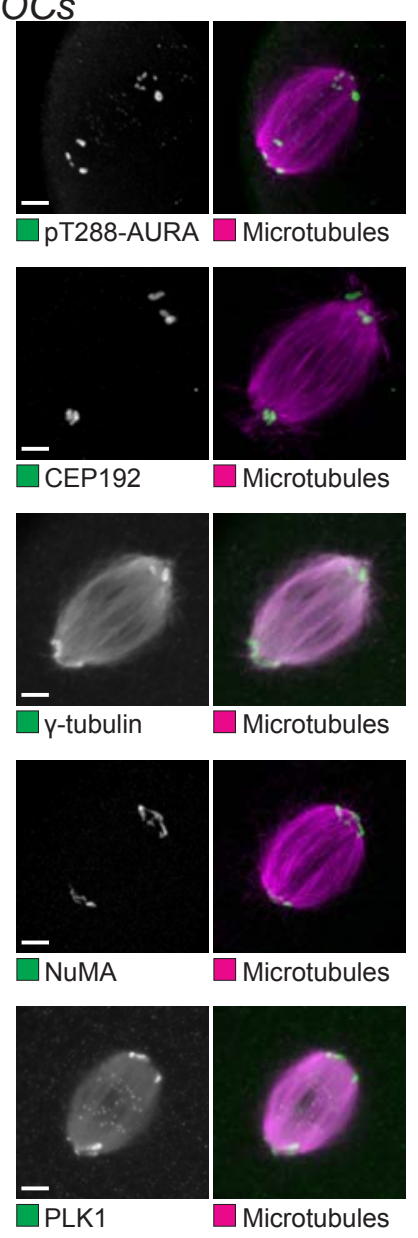
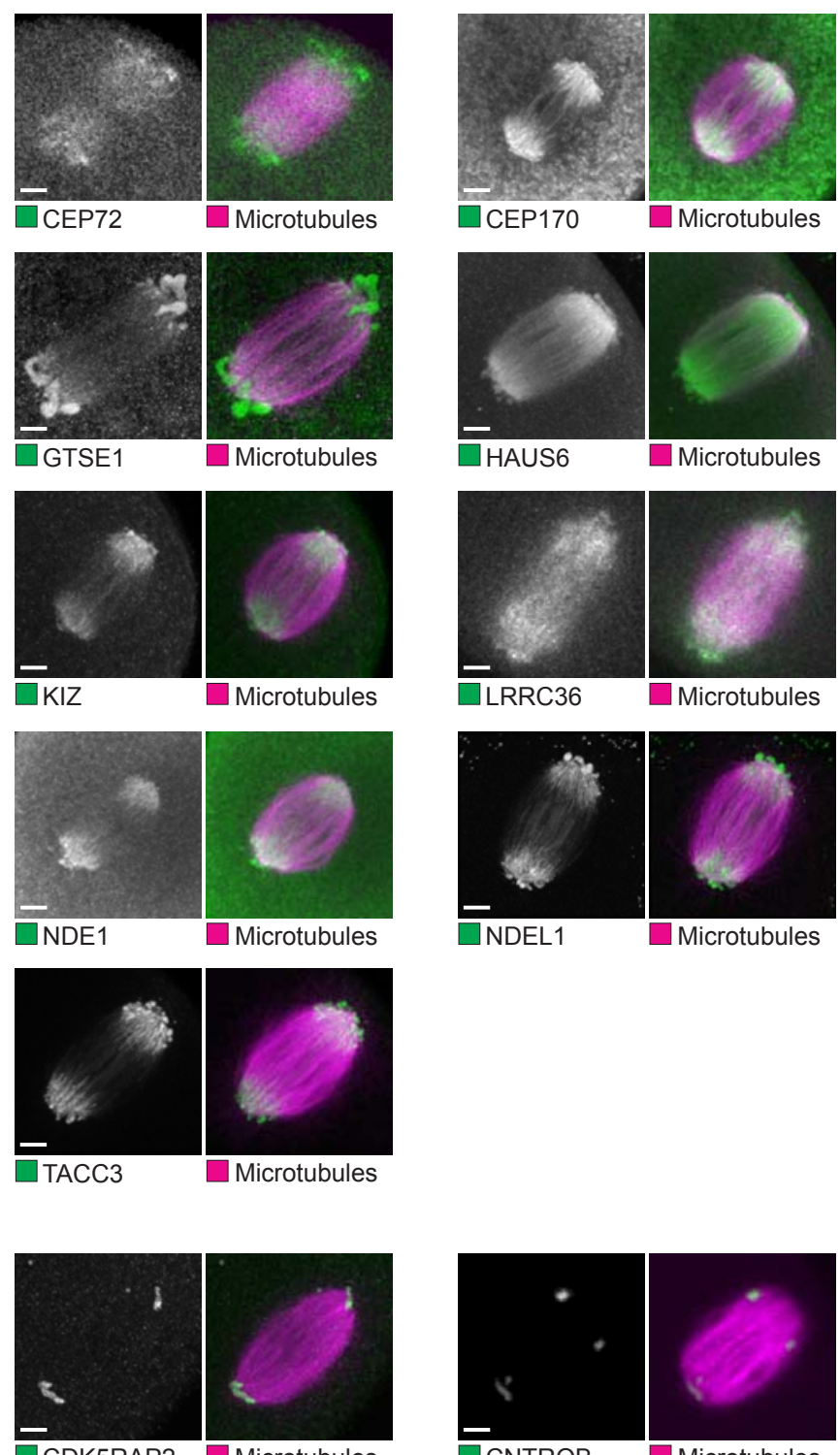

$\square$ CEP170 $\square$ Microtubules
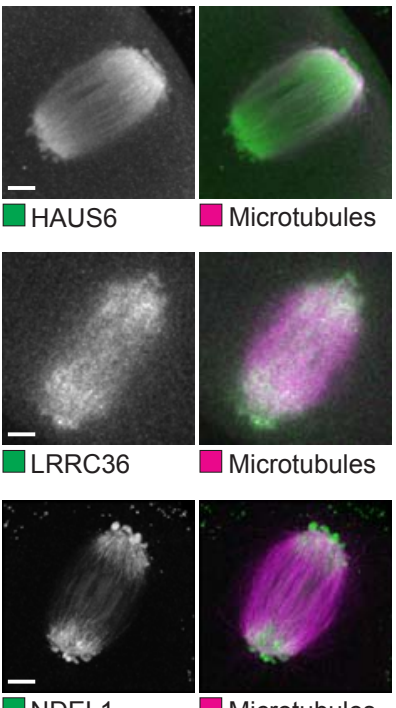

NDEL1

$\square$ Microtubules
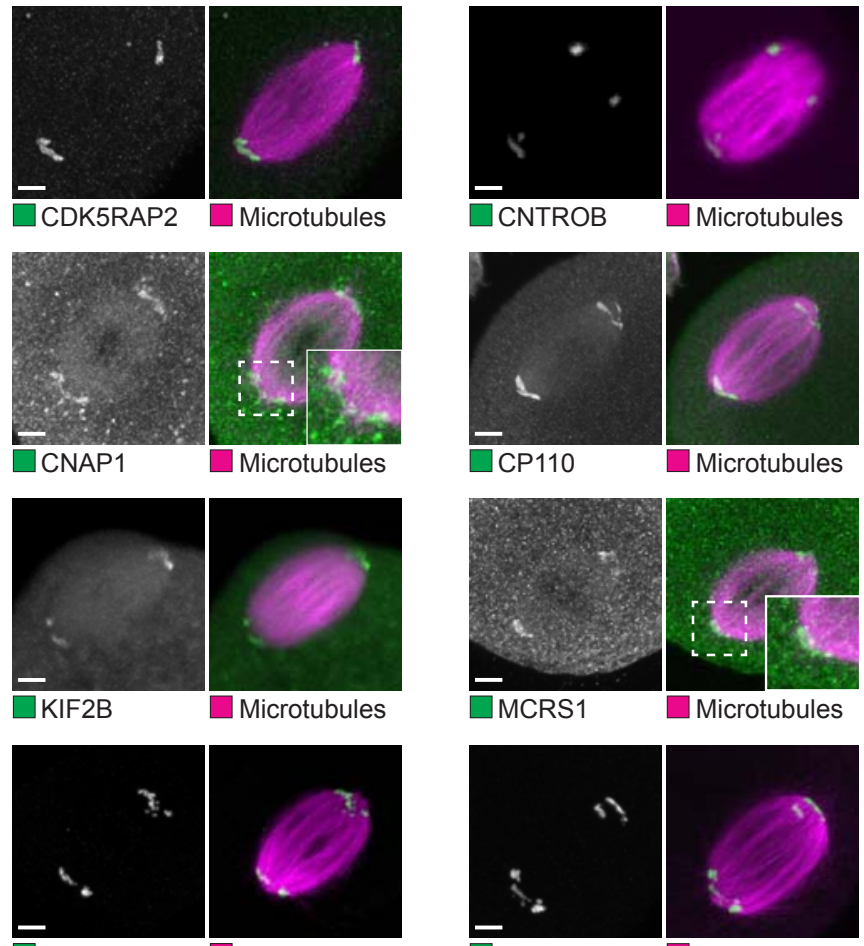

- pS395-NuMA @ Microtubules

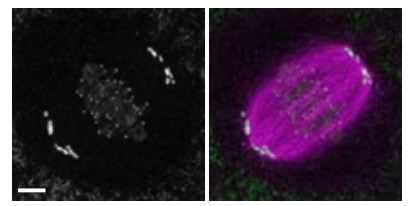

pT210-PLK1 Microtubules

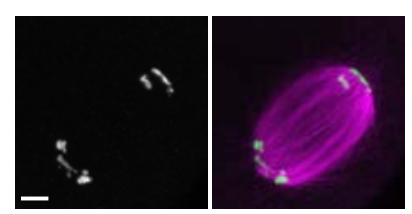

$\square$ pT2055-NuMA Microtubules

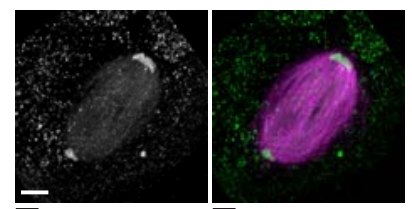

PLK4 

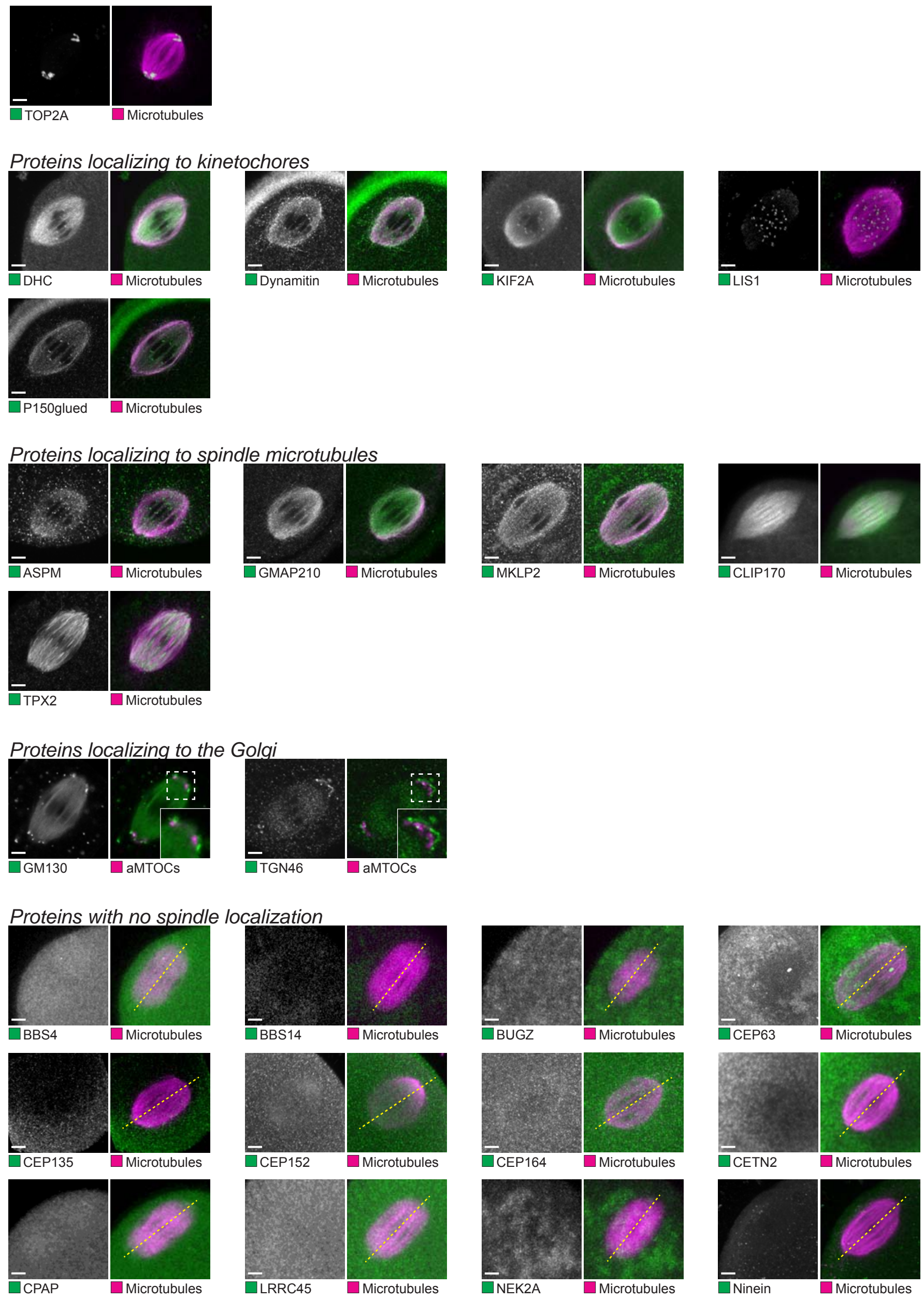

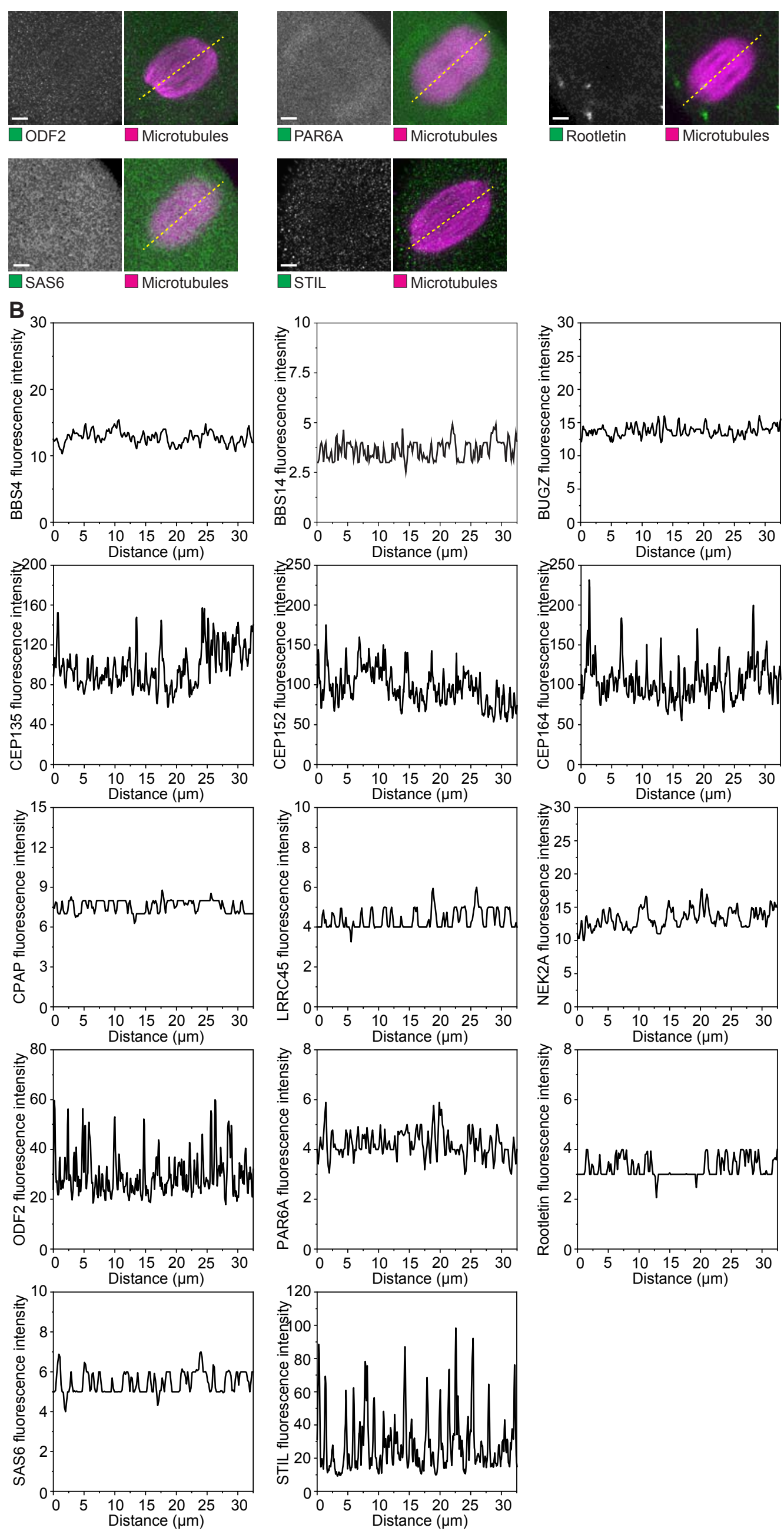

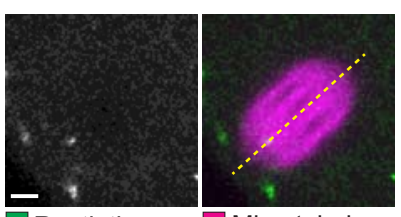

$\square$ Rootletin
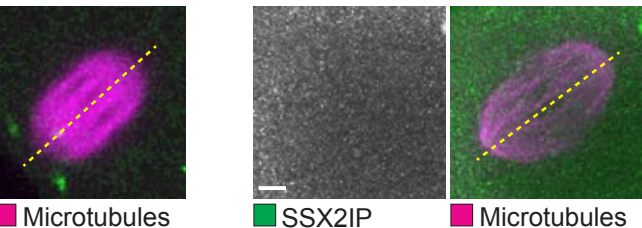

SSX2IP
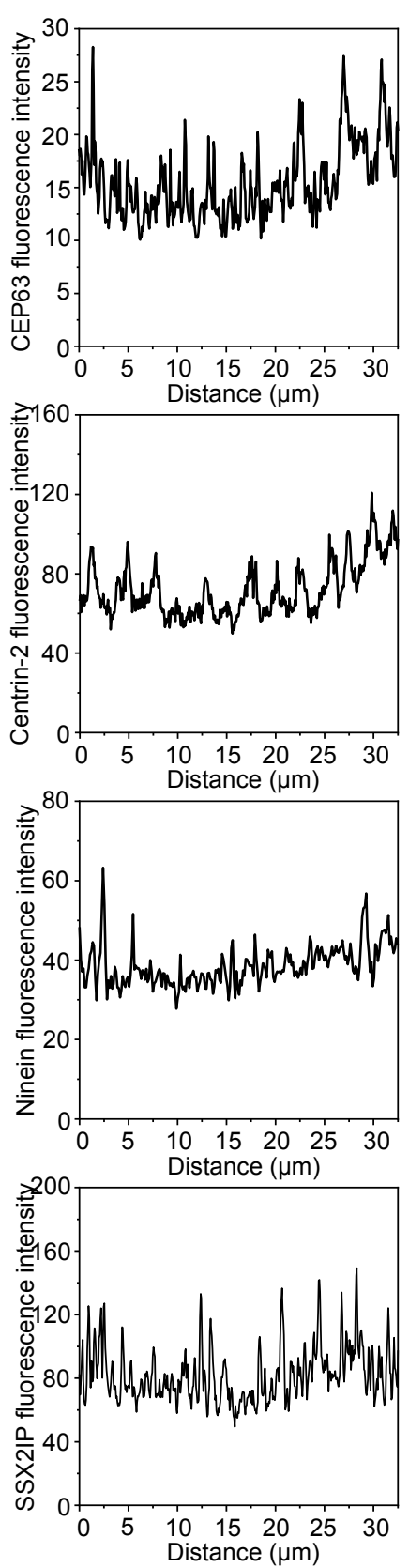
Fig. S1 Systematic analysis of the localization of centrosomal and spindle pole-related proteins on acentrosomal spindles in mouse oocytes. (A) Fluorescence images of AKAP450, CAMSAP3, CEP72, CEP170, CHC17, chTOG, GTSE1, HAUS6, HOOK3, KANSL3, KIZ, LRRC36, MCAK, MYO10, NDE1, NDEL1, PCM1, SPDL1, TACC3, AURA, pT288-AURA, CDK5RAP2, Centrobin, CEP120, CEP192, CNAP1, CP110, DISC1, $\gamma$-tubulin, KIF2B, MCRS1, NEDD1, NuMA, pS395-NuMA, pT2055-NuMA, Pericentrin, PLK1, pT210-PLK1, PLK4, TOP2A, DHC, Dynamitin, KIF2A, LIS1, P150glued, ASPM, GMAP210, MKLP2, CLIP170, TPX2, GM130, TGN46, BBS4, BBS14, BUGZ, CEP63, CEP135, CEP152, CEP164, CETN2, CPAP, LRRC45, NEK2A, Ninein, ODF2, PAR6A, Rootletin, SSX2IP, SAS6 and STIL in mouse metaphase I oocytes. Green, protein of interest; magenta, microtubules ( $\alpha$-tubulin or mCherryMAP4-MTBD) or aMTOCs (pericentrin). Inset is the magnification of region outlined by the dashed line box. Note that the prominent signal on spindle microtubules for PLK1 and GM130 was due to the anti-mouse secondary antibody. All antibodies used for immunofluorescence of proteins with no detectable localization in mouse metaphase I oocytes had been tested to work in the surrounding cumulus cells. Yellow lines mark positions corresponding to fluorescence profiles in (B). (B) Fluorescence profiles of BBS4, BBS14, BUGZ, CEP63, CEP135, CEP152, CEP164, Centrin-2, CPAP, LRRC45, NEK2A, Ninein, ODF2, PAR6A, Rootletin, SSX2IP, SAS6 and STIL along lines passing through the spindles in mouse metaphase I oocytes. Scale bars, $5 \mu \mathrm{m}$. 
Figure S2
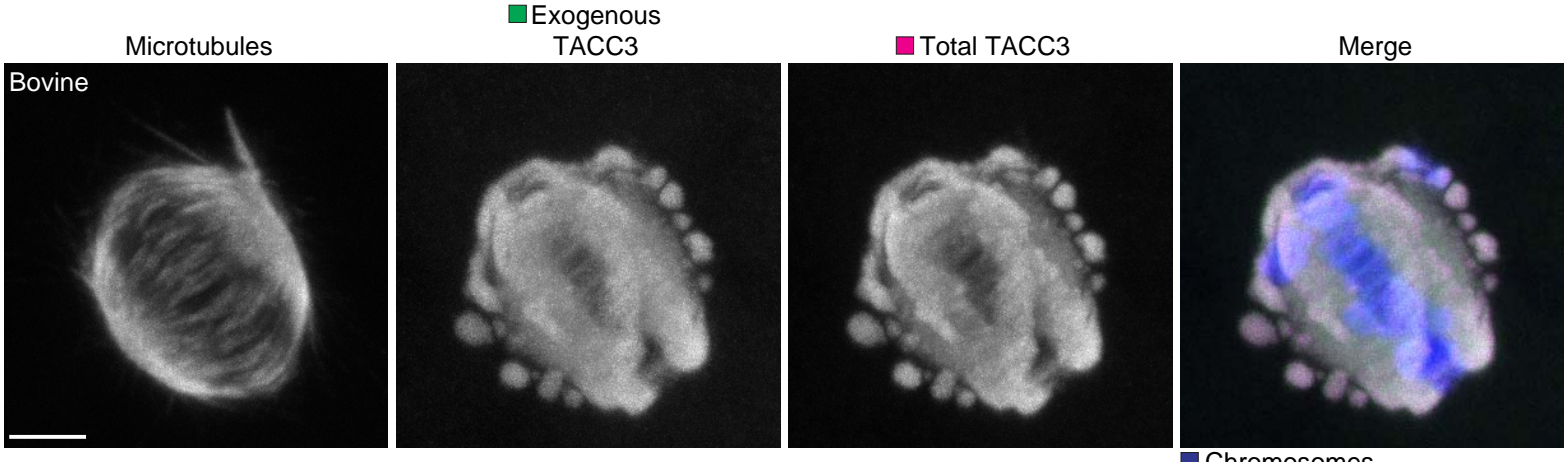

Chromosomes 
Fig. S2 The anti-TACC3 is specific in bovine oocytes. Immunofluorescence images of bovine metaphase I oocytes expressing TACC3-mClover3. Gray, microtubules ( $\alpha$-tubulin); green, exogenous TACC3 (GFP); magenta, total (exogenous and endogenous) TACC3; blue, chromosomes (Hoechst). Scale bar, $5 \mu \mathrm{m}$. 
Figure S3

A

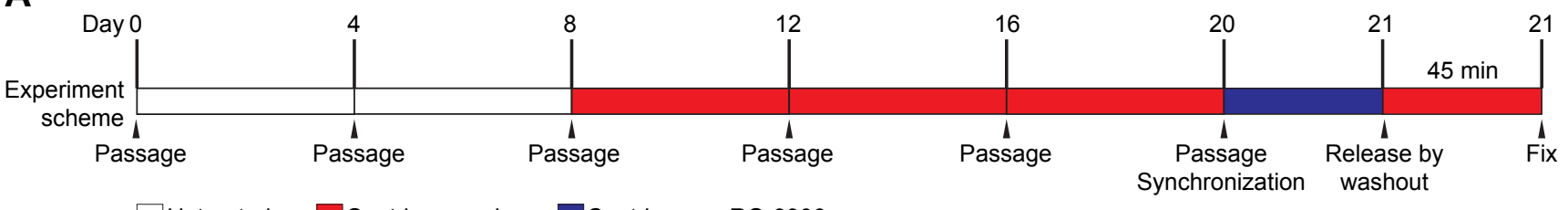

$\square$ Untreated $\square$ Centrinone only $\square$ Centrinone + RO-3306

B
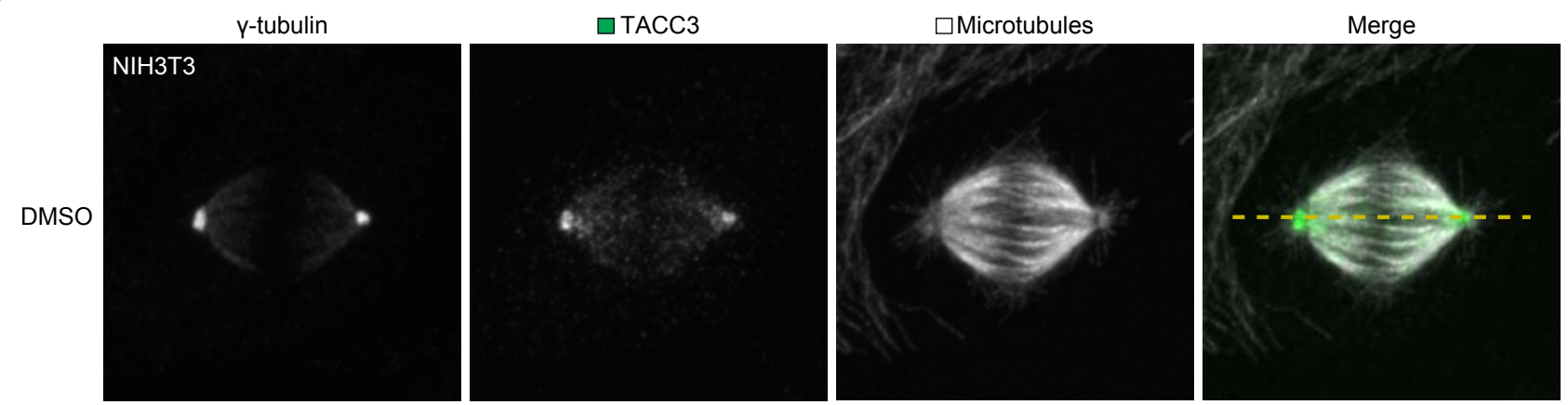

C
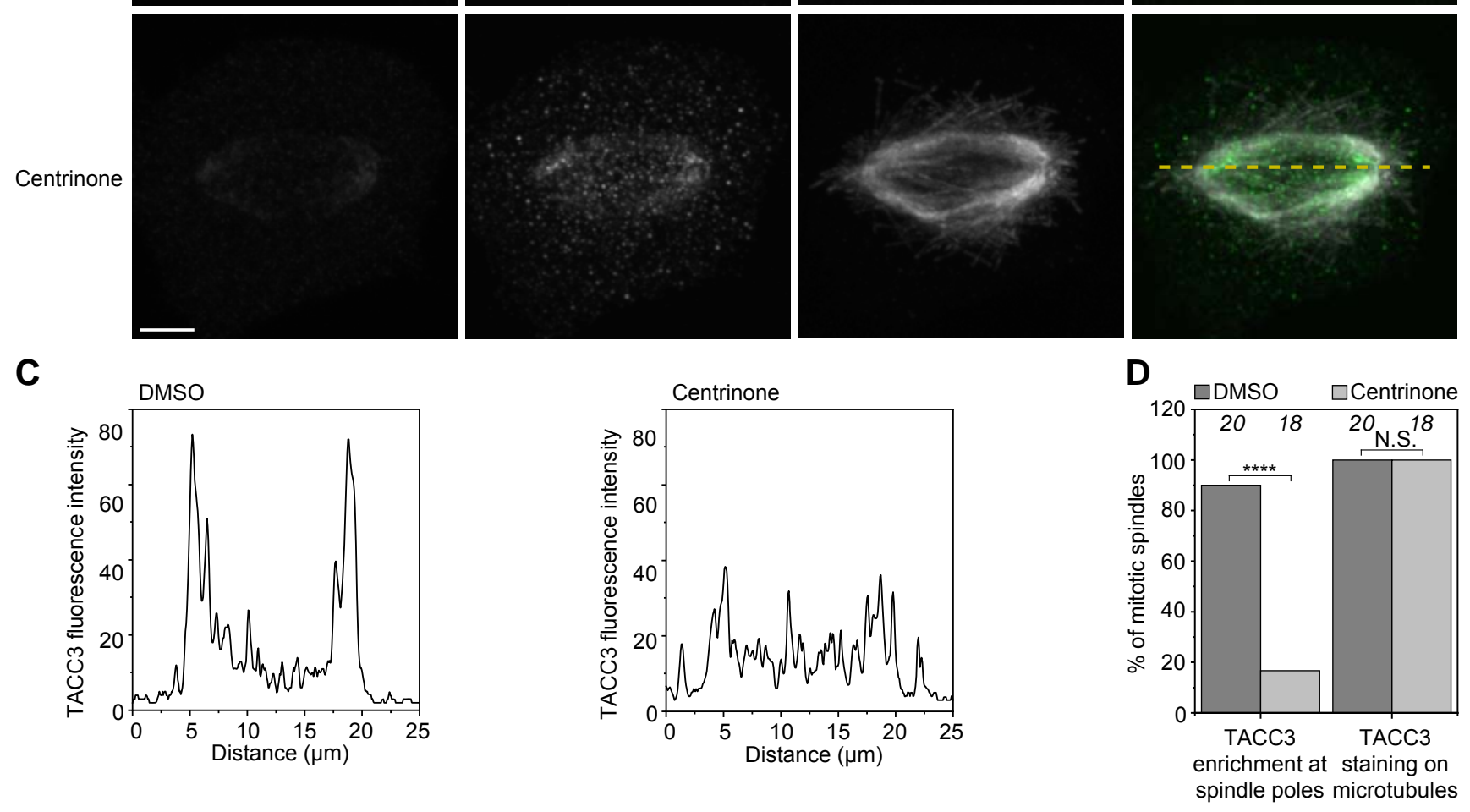

D

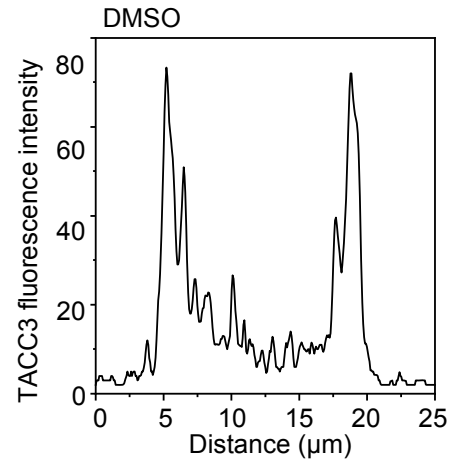


Fig. S3 The LISD is absent from mitotic spindles in centrosome-depleted mouse embryonic fibroblasts. (A) Schematic of centrosome depletion in NIH3T3 cells. (B) Immunofluorescence images of DMSO- and $300 \mathrm{nM}$ centrinone-treated NIH3T3 cells. Green, TACC3; gray, microtubules ( $\alpha$-tubulin); magenta, chromosomes (Hoechst). Yellow lines mark positions corresponding to fluorescence profiles in (C). (C) Fluorescence profiles of TACC3 along lines passing through the spindles in DMSO- and $300 \mathrm{nM}$ centrinone-treated NIH3T3 cells. (D) Quantification of TACC3 localization in DMSO- and $300 \mathrm{nM}$ centrinone-treated NIH3T3 cells. The number of analyzed cells is specified in italics. Note that centrinone-treated cells with TACC3 enrichment at spindle poles were positive for $\gamma$-tubulin, suggesting that these cells still possess their centrosomes. Scale bars, $5 \mu \mathrm{m}$. 
Figure S4
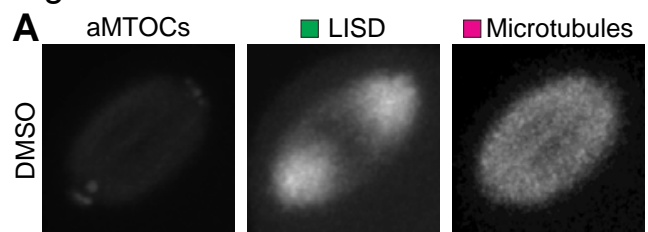

Merge

B
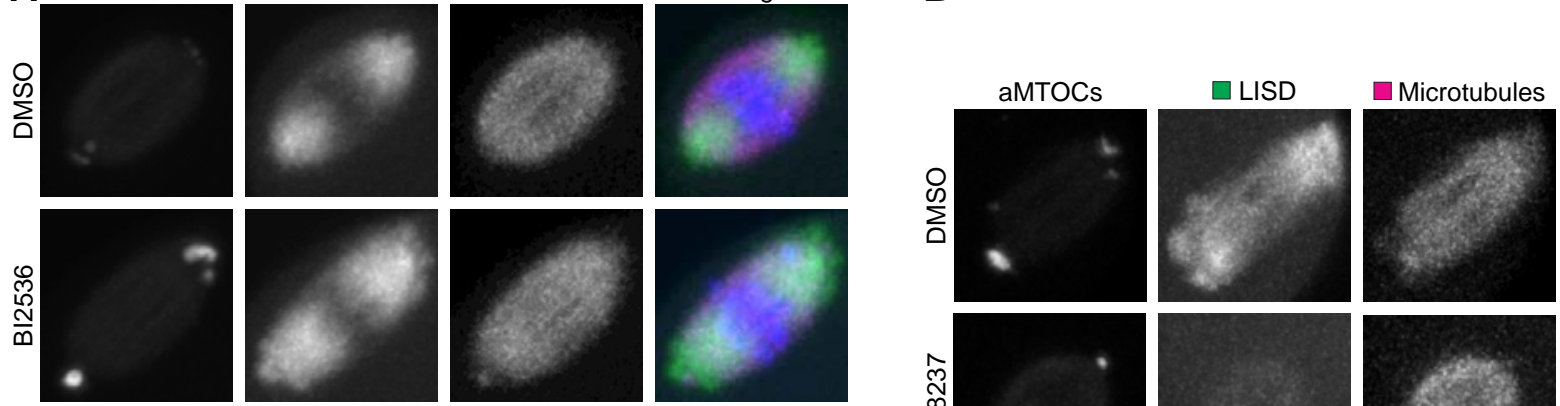

Merge
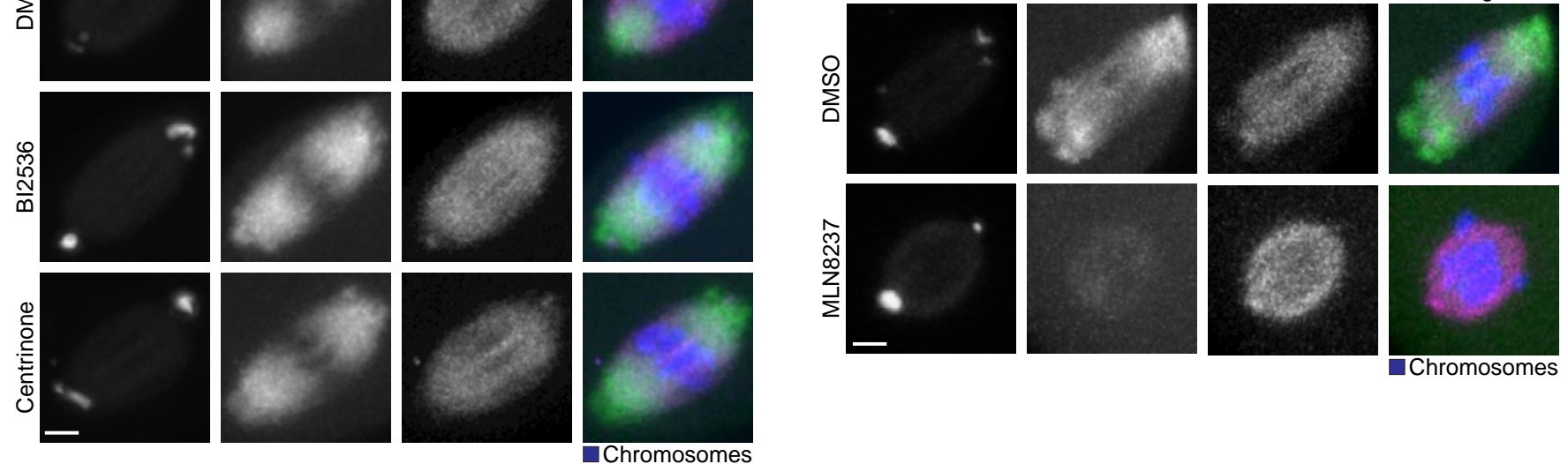

CAcute MLN8237 addition
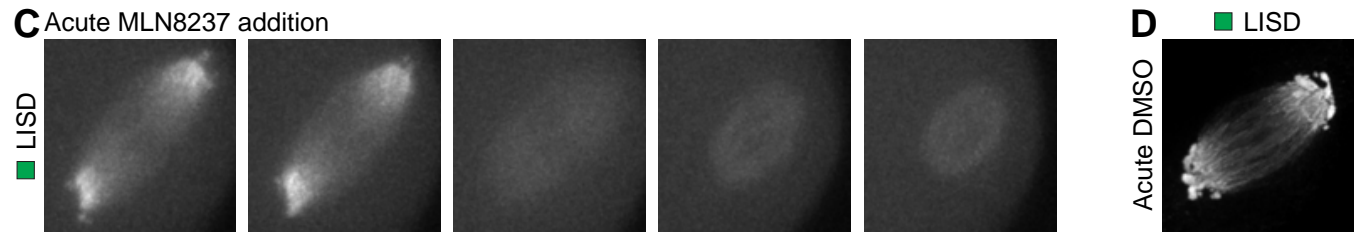

$\square$ Microtubules
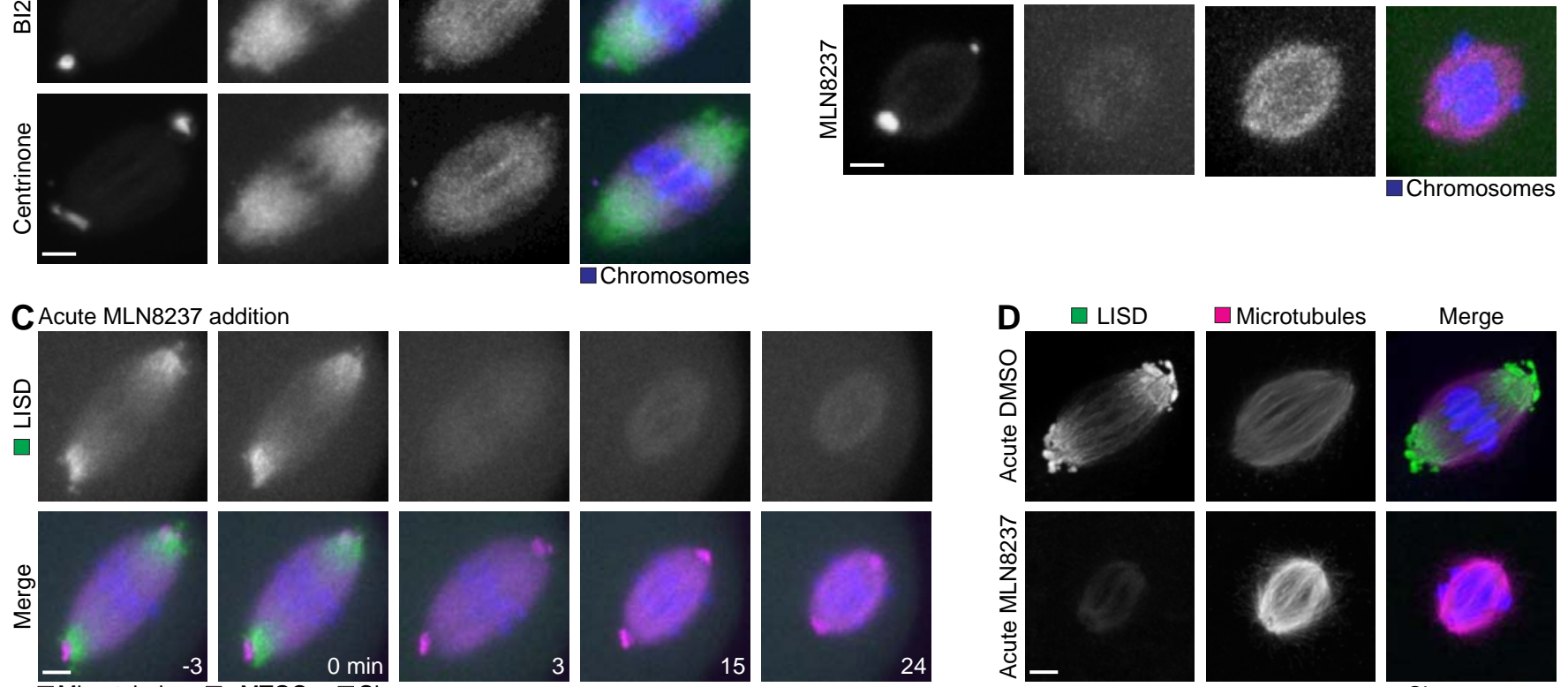

Merge
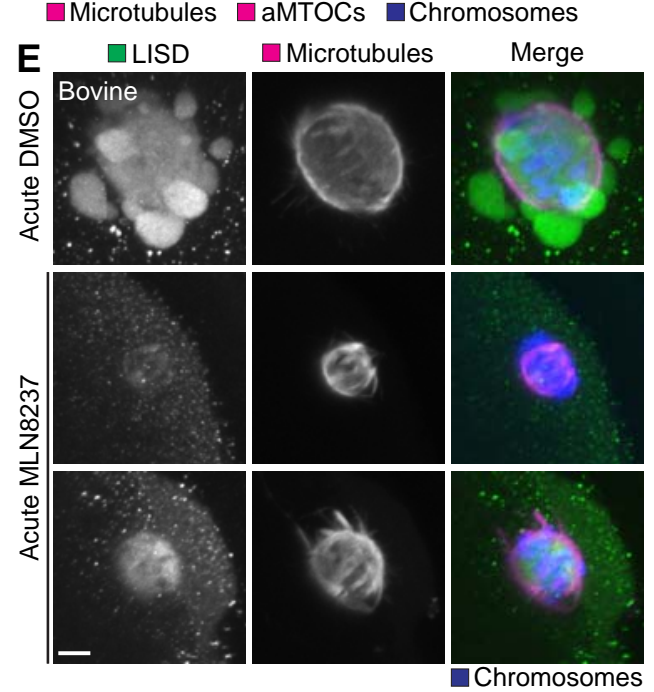
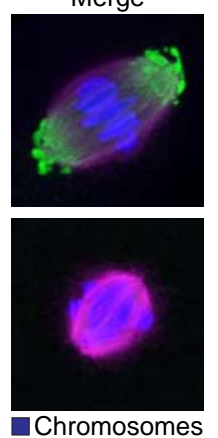

Chromosomes 
Fig. S4 AURA, but not PLK1 or PLK4 drives the assembly of the LISD. (A) Still images from time-lapse movies of DMSO-, $100 \mathrm{nM}$ BI2536 (PLK1 inhibitor)- and $5 \mu \mathrm{M}$ centrinone (PLK4 inhibitor)-treated mouse metaphase I oocytes. Gray, aMTOCs (CEP192-mScarlet); green, LISD (TACC3-mClover3); magenta, microtubules (3×CyOFP-MAP4-MTBD); blue, chromosomes (H2B-miRFP). (B) Still images from time-lapse movies of DMSO- and $500 \mathrm{nM}$ MLN8237 (AURA inhibitor)-treated mouse metaphase I oocytes. Gray, aMTOCs (CEP192-mScarlet); green, LISD (TACC3-mClover3); magenta, microtubules (3×CyOFP-MAP4-MTBD); blue, chromosomes (H2B-miRFP). (C) Still images from time-lapse movies of acutely MLN8237treated mouse metaphase I oocytes. Green, LISD (TACC3-mClover3); magenta, microtubules (3×CyOFP-MAP4-MTBD) and aMTOCs (CEP192-mScarlet); blue, chromosomes (H2B-miRFP). Time is given as minutes after $500 \mathrm{nM}$ MLN8237 addition. (D) Immunofluorescence images of acutely DMSO- and $500 \mathrm{nM}$ MLN8237-treated mouse metaphase I oocytes. Green, LISD (TACC3); magenta, microtubules ( $\alpha$-tubulin); blue, chromosomes (Hoechst). (E) Immunofluorescence images of acutely DMSO- and $500 \mathrm{nM}$ MLN8237-treated bovine metaphase I oocytes. Green, LISD (TACC3); magenta, microtubules ( $\alpha$-tubulin); blue, chromosomes (Hoechst). Scale bars, $5 \mu \mathrm{m}$. 
Figure S5

A

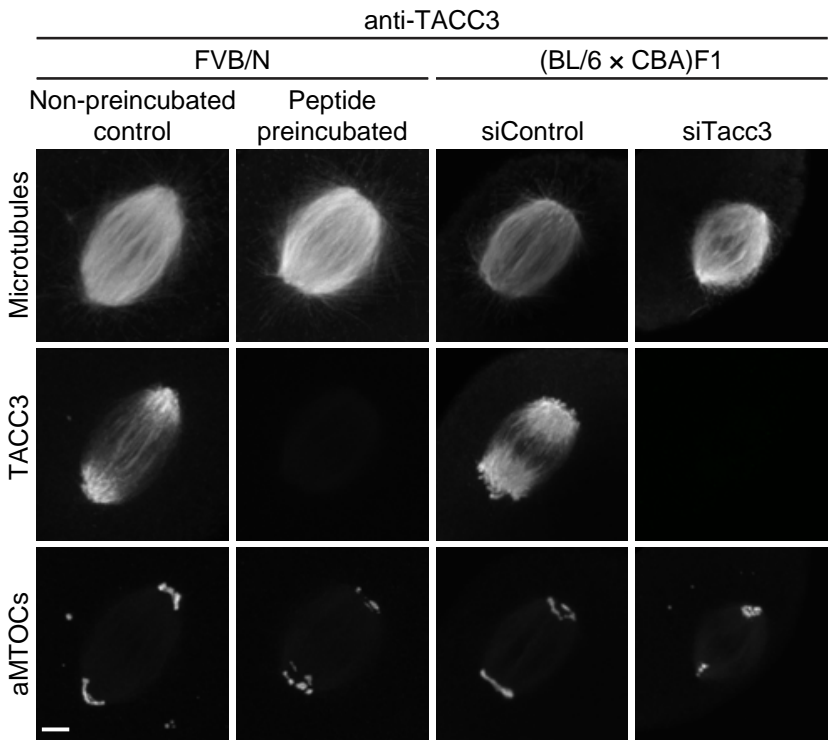

B
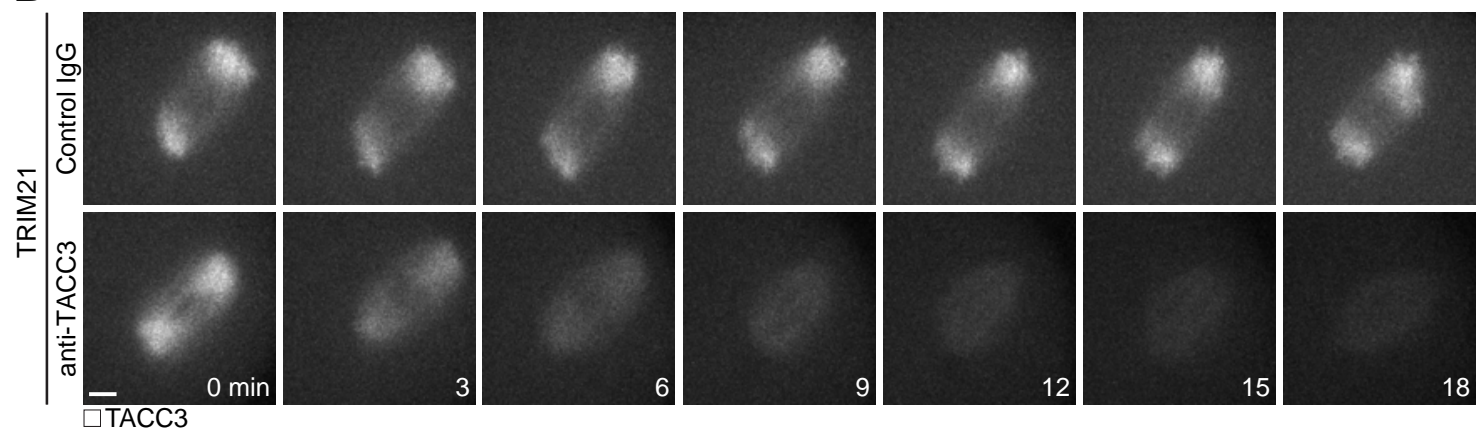

C

D
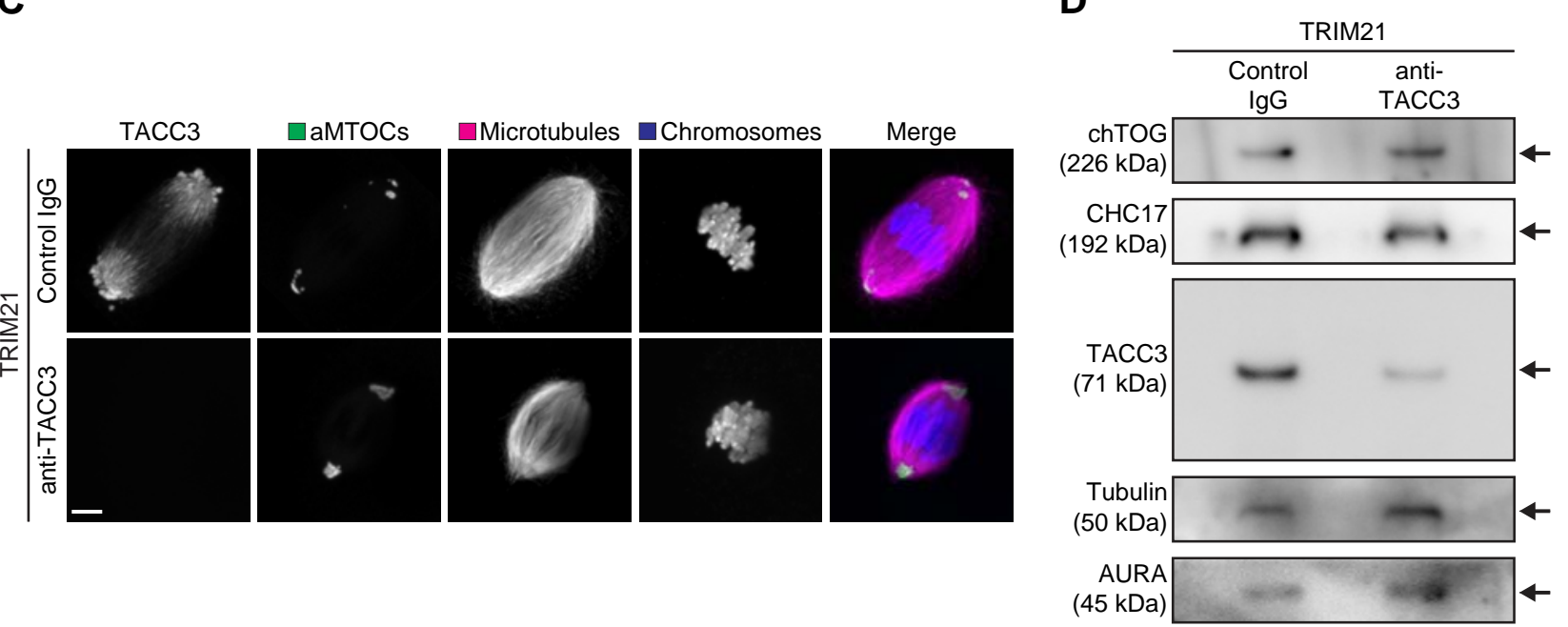
Fig. S5 Specific depletion of endogenous TACC3 in mouse oocytes using Trim-Away. (A) Immunofluorescence images of non-preincubated, TACC3 peptide preincubated, control siRNAinjected and TACC3 siRNA-injected mouse metaphase I oocytes. (B) Still images from time-lapse movies of control and TACC3-depleted mouse metaphase I oocytes expressing TACC3mClover3. Gray, TACC3. (C) Immunofluorescence images of the metaphase I spindle in control and TACC3-depleted mouse metaphase I oocytes. Gray, TACC3; green, aMTOCs (pericentrin); magenta, microtubules ( $\alpha$-tubulin); blue, chromosomes (Hoechst). (D) Immunoblots of control and TACC3-depleted mouse metaphase I oocytes for chTOG, CHC17, TACC3, $\alpha$-tubulin and AURA. Black arrows indicate the band corresponding to each protein. Scale bars, $5 \mu \mathrm{m}$. 
Figure S6
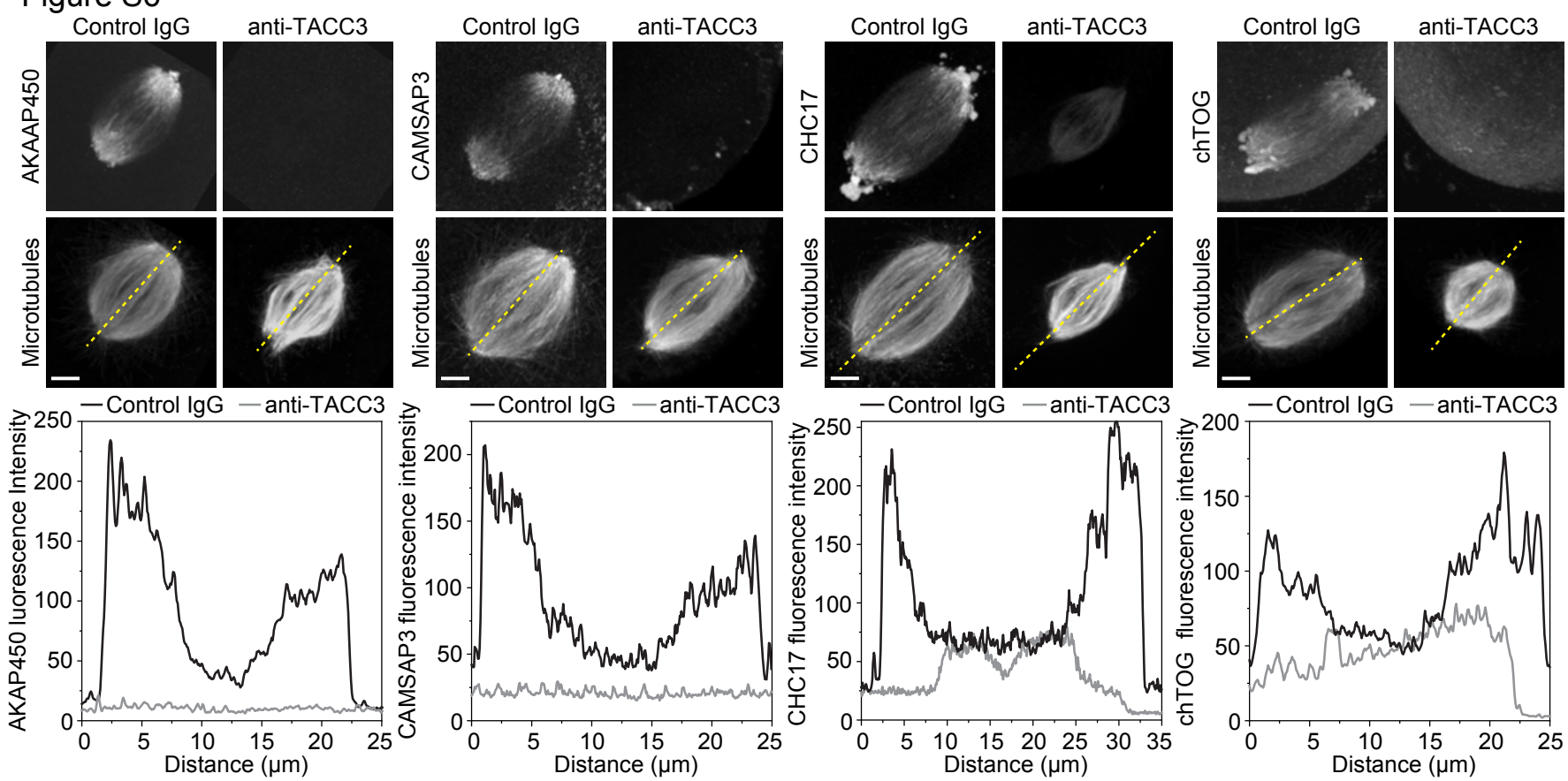

- Control IgG - anti-TACC3
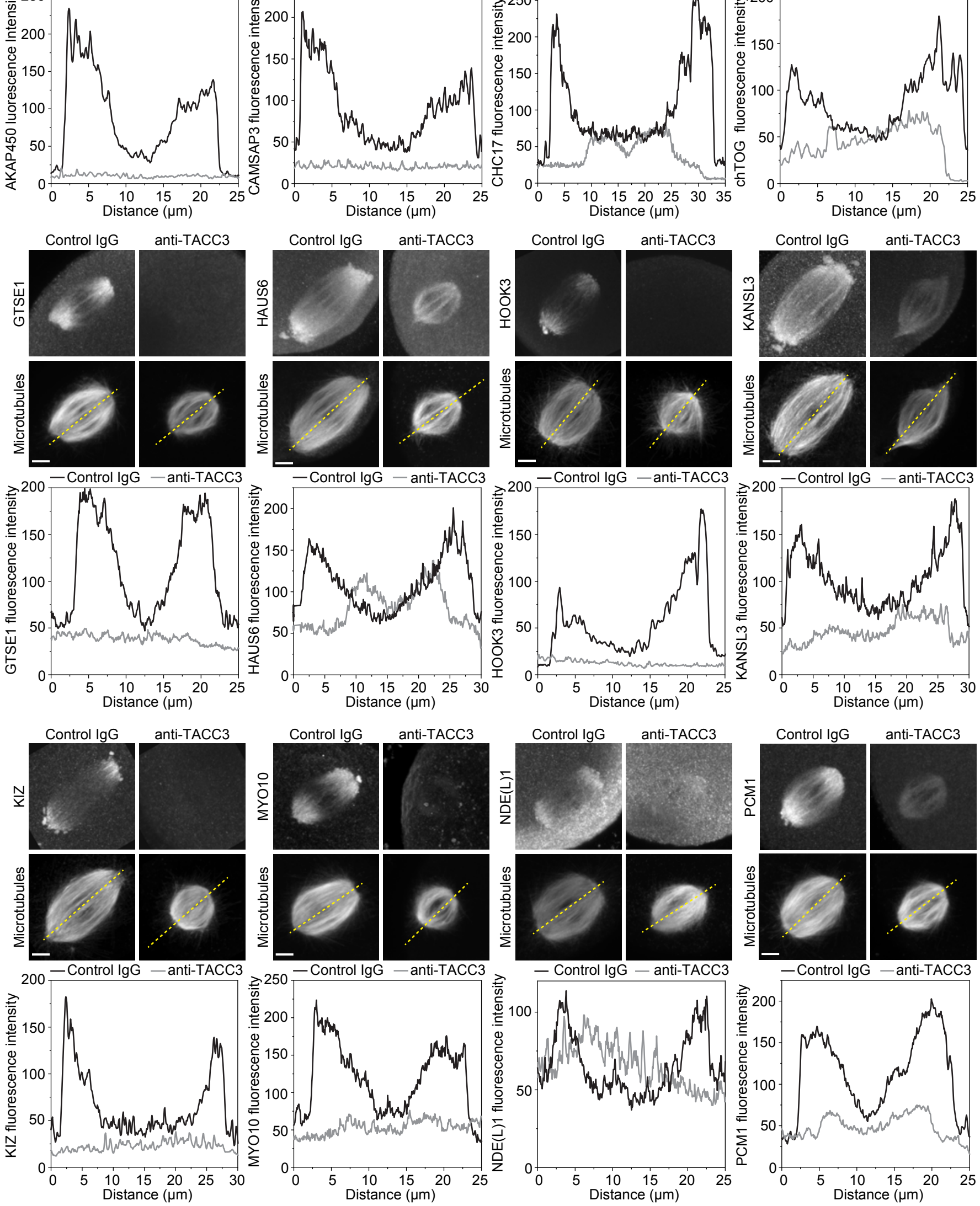
Fig. S6 Depletion of TACC3 disrupts the assembly of the LISD. Immunofluorescence images of AKAP450, CAMSAP3, CHC17, chTOG, GTSE1, HAUS6, HOOK3, KANSL3, KIZ, MYO10, NDE(L)1 and PCM1 in control and TACC3-depleted mouse metaphase I oocytes. Yellow lines mark positions corresponding to the fluorescence profiles. Scale bars, $5 \mu \mathrm{m}$. 
Figure S7
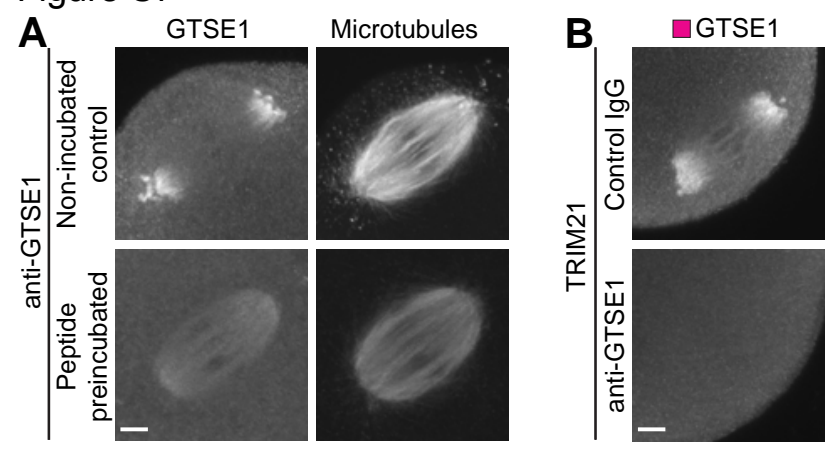

$\square$ LISD Merge

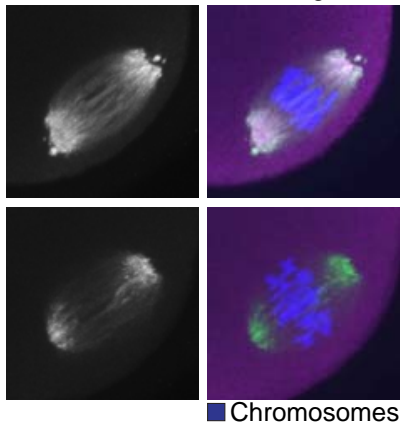


Fig. S7 GTSE1 is not essential for the assembly of the LISD. (A) Immunofluorescence images of non-preincubated and GTSE1 peptide preincubated mouse metaphase I oocytes. (B) Immunofluorescence images of control and GTSE1-depleted mouse metaphase I oocytes. Gray, GTSE1. Green, LISD (TACC3); magenta, microtubules ( $\alpha$-tubulin); blue, chromosomes (Hoechst). Scale bars, $5 \mu \mathrm{m}$. 
Figure S8
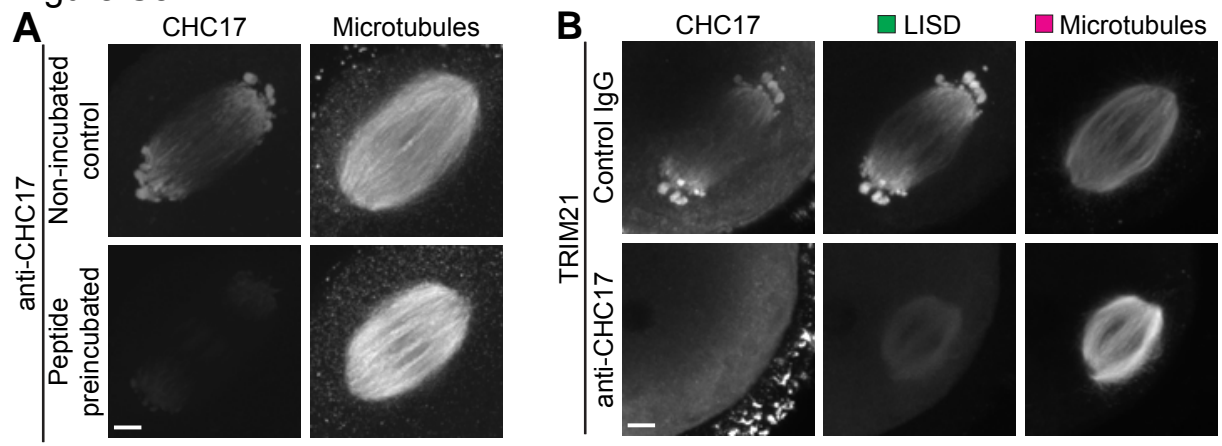

Merge
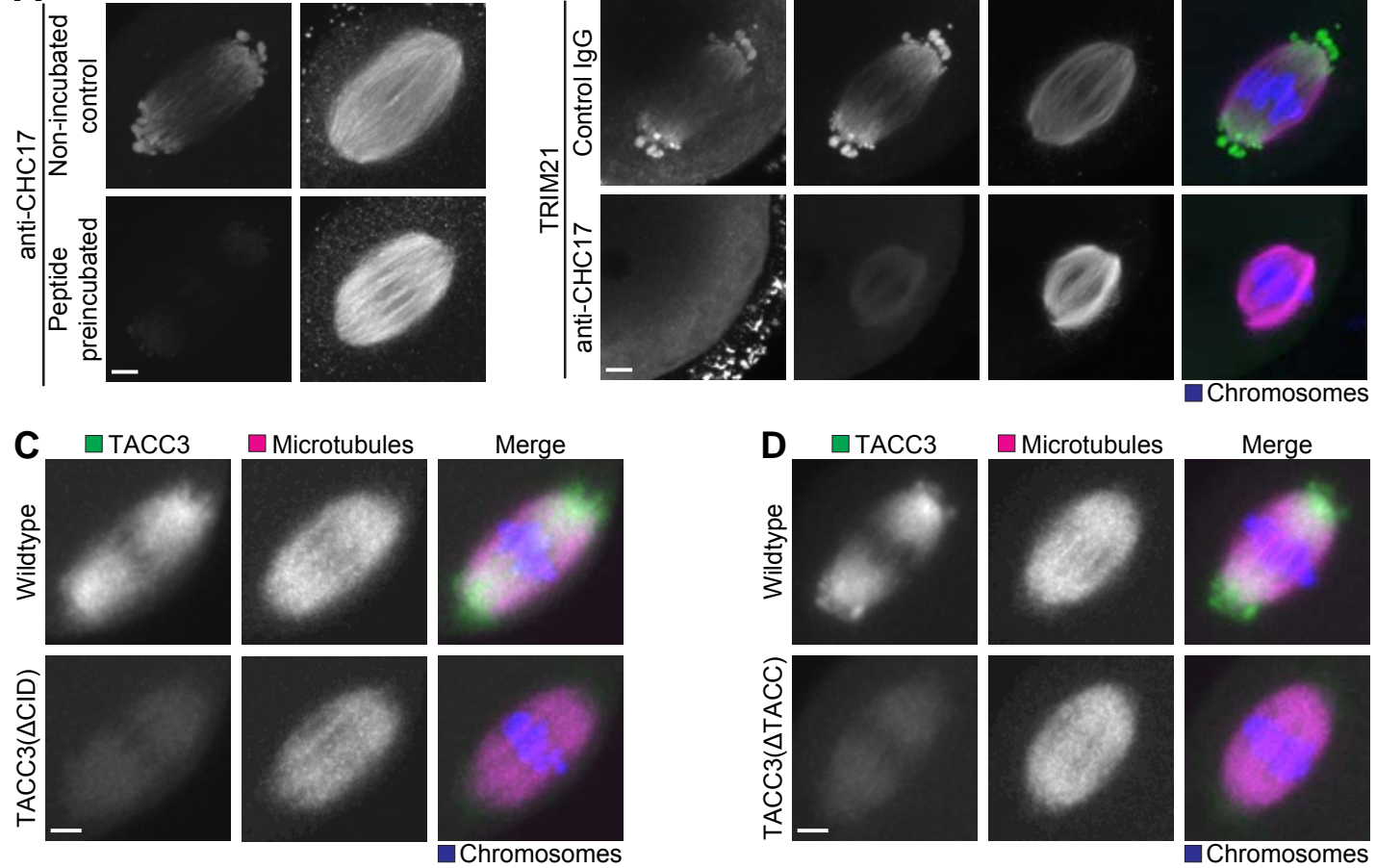

Merge
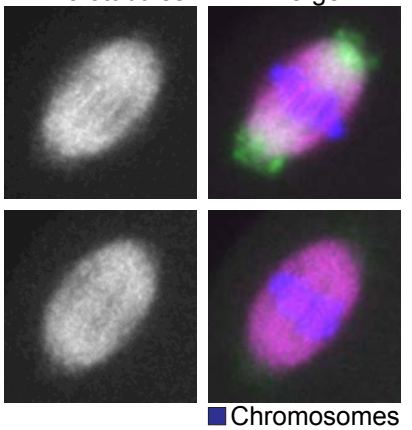
Fig. S8 CHC17 is required for the assembly of the LISD. (A) Immunofluorescence images of non-preincubated and $\mathrm{CHC17}$ peptide preincubated mouse metaphase I oocytes. (B) Immunofluorescence images of control and $\mathrm{CHC} 17$-depleted mouse metaphase I oocytes. Gray, CHC17; green, LISD (TACC3); magenta, microtubules ( $\alpha$-tubulin); blue, chromosomes (Hoechst). (C) Still images from time-lapse movies of mouse metaphase I oocytes expressing TACC3-mClover3 and TACC3( $\triangle \mathrm{CID})(\triangle \mathrm{aa} 522-577)$-mClover3. The clathrin-interacting domain (CID) is required for TACC3's interaction with $\mathrm{CHC} 17$ (20). Green, TACC3 constructs; magenta, microtubules $(3 \times$ CyOFP-MAP4-MTBD); blue, chromosomes (H2B-miRFP). (D) Still images from time-lapse movies of mouse metaphase I oocytes expressing TACC3-mClover3 and TACC3( $\triangle \mathrm{TACC}$ ) (aa 1-593)-mClover3. The TACC domain is required for TACC3 to form the microtubule interaction surface with $\mathrm{CHC17}$ (20). Green, TACC3 constructs; magenta, microtubules (3×CyOFP-MAP4-MTBD); blue, chromosomes (H2B-miRFP). Scale bars, $5 \mu \mathrm{m}$. 
Figure S9

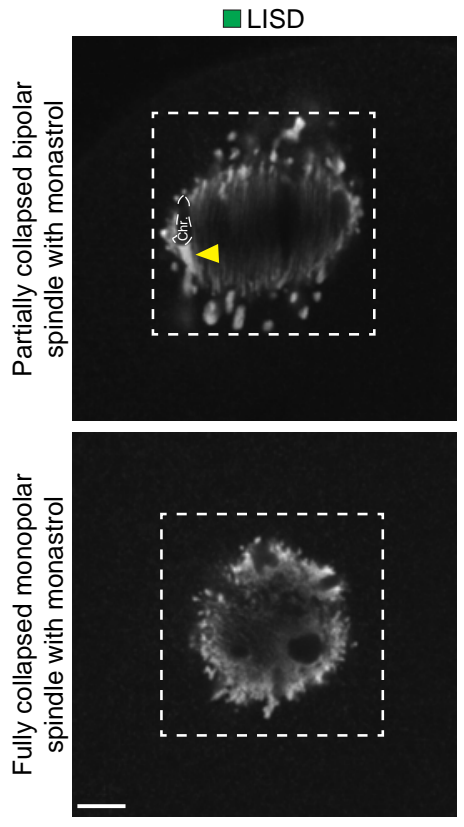

Microtubules
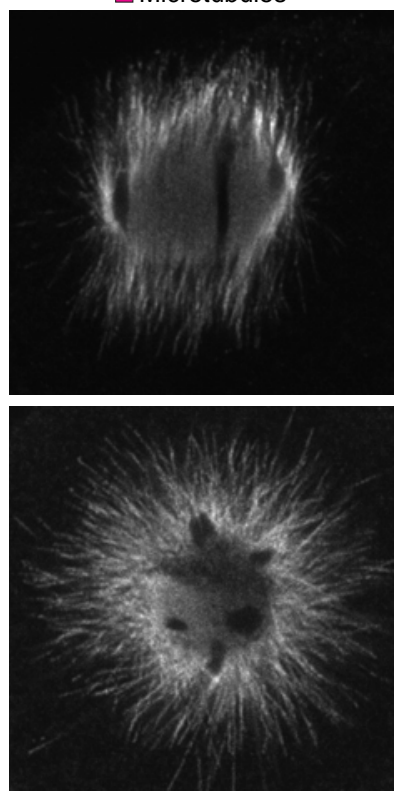

Merge
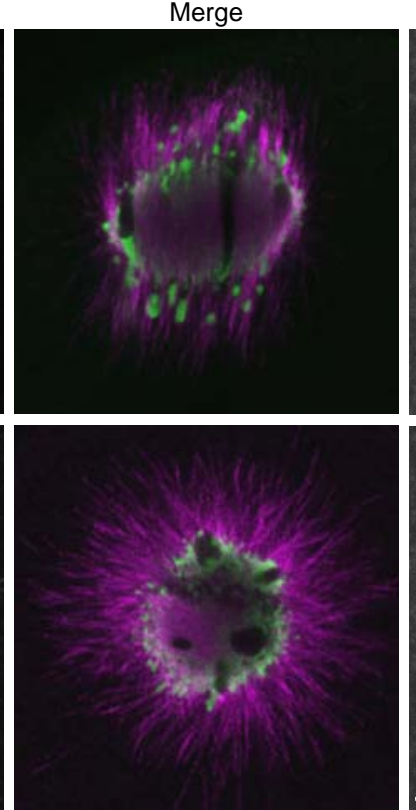

Zoom \& Enhanced brightness
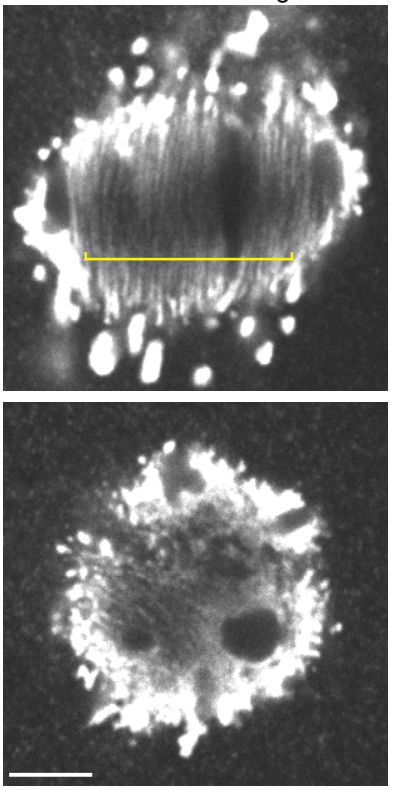
Fig. S9 The LISD encapsulates K-fibers and a subset of spindle microtubules. Immunofluorescence images of mouse metaphase I spindles fixed early and late (before and after collapsing into monopolar spindles) after monastrol treatment. Mouse metaphase I oocytes were treated with monastrol to induce spindle shortening and broadening to better resolve the dense microtubules at the meiotic spindle poles. Green, LISD (TACC3); magenta, microtubules $(\alpha-$ tubulin). Insets are magnifications of regions outlined by dashed line boxes. Yellow arrowhead indicates a K-fiber. Yellow bracket indicates non-kinetochore-bound microtubules encapsulated by the LISD. Scale bars, $5 \mu \mathrm{m}$. 
Figure S10
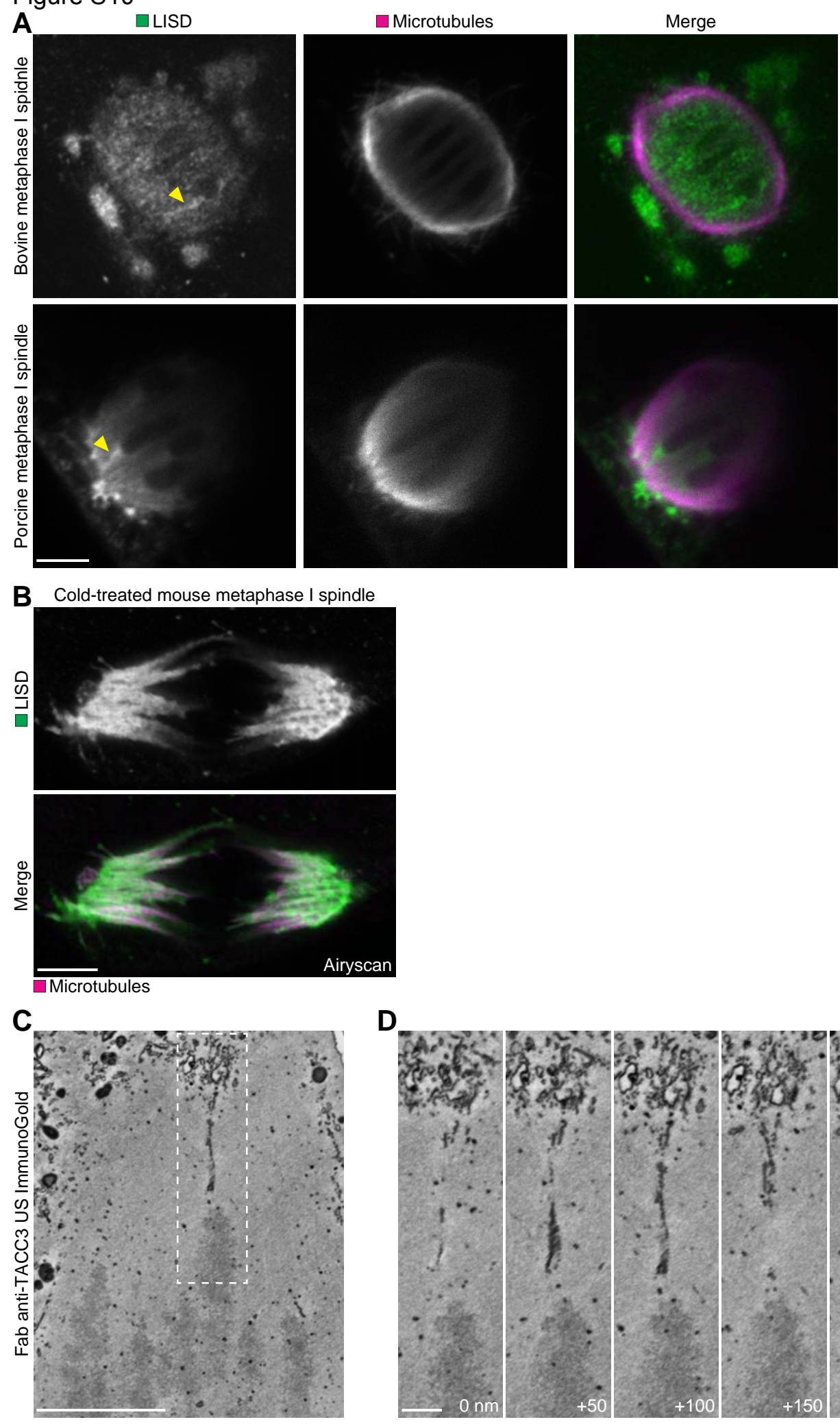

\section{D}

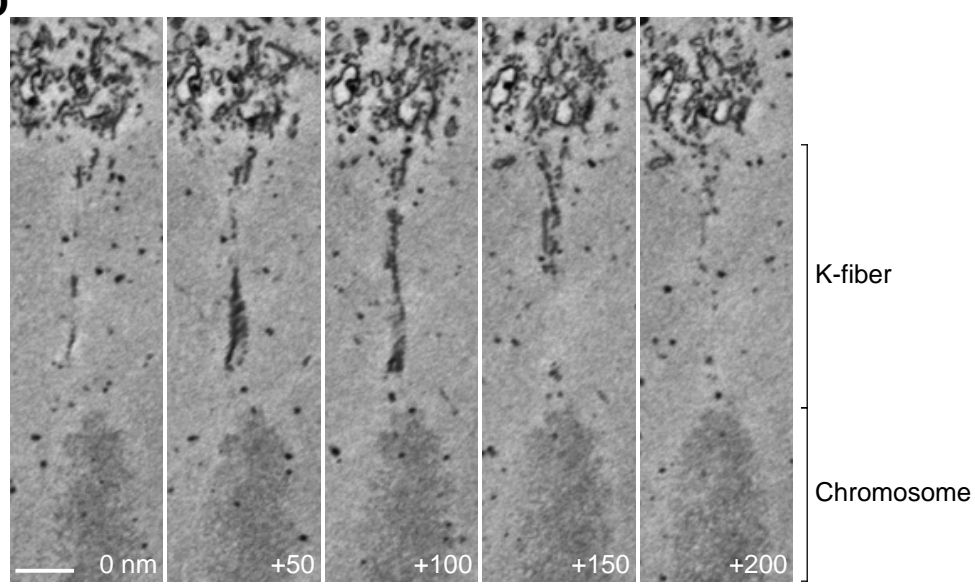


Fig. S10 The LISD extends towards the spindle midzone and is enriched on K-fibers. (A) Single confocal sections of the metaphase I spindles in bovine and porcine oocytes. Green, LISD (TACC3). Magenta, microtubules ( $\alpha$-tubulin). Yellow arrowheads indicate K-fibers. (B) A single confocal section of a cold-treated mouse metaphase I spindle. Green, LISD (TACC3); magenta, microtubules ( $\alpha$-tubulin). (C) A single section of FIB-SEM of mouse early metaphase I oocytes injected with Fab anti-TACC3 US ImmunoGold. (D) Single sections of the region outlined by dashed line box in (B). Scale bar, $1 \mu \mathrm{m}$. Scale bars, $5 \mu \mathrm{m}$ unless otherwise specified. 
Figure S11
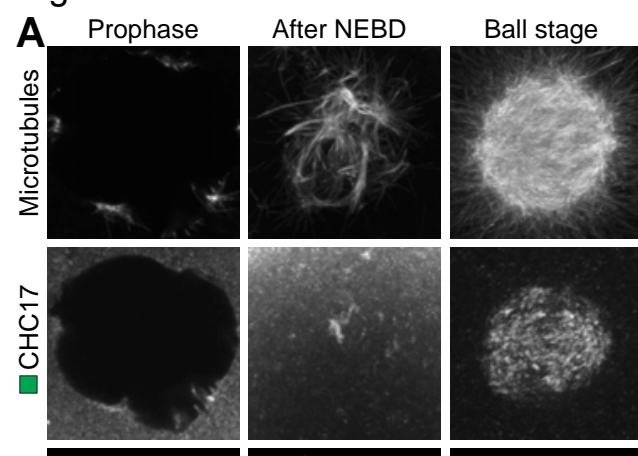

Bipolarization

Metaphase I
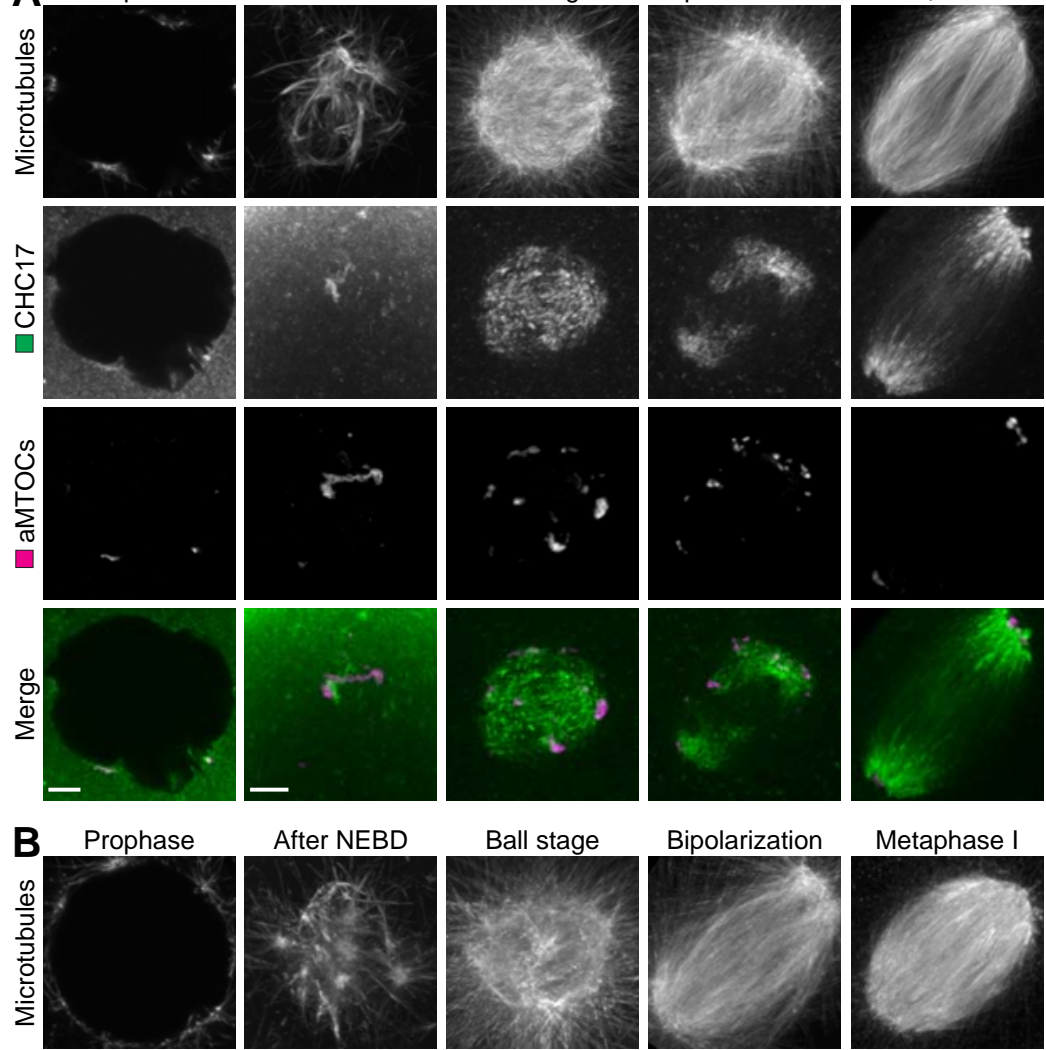

Ball stage

Bipolarization

Metaphase I
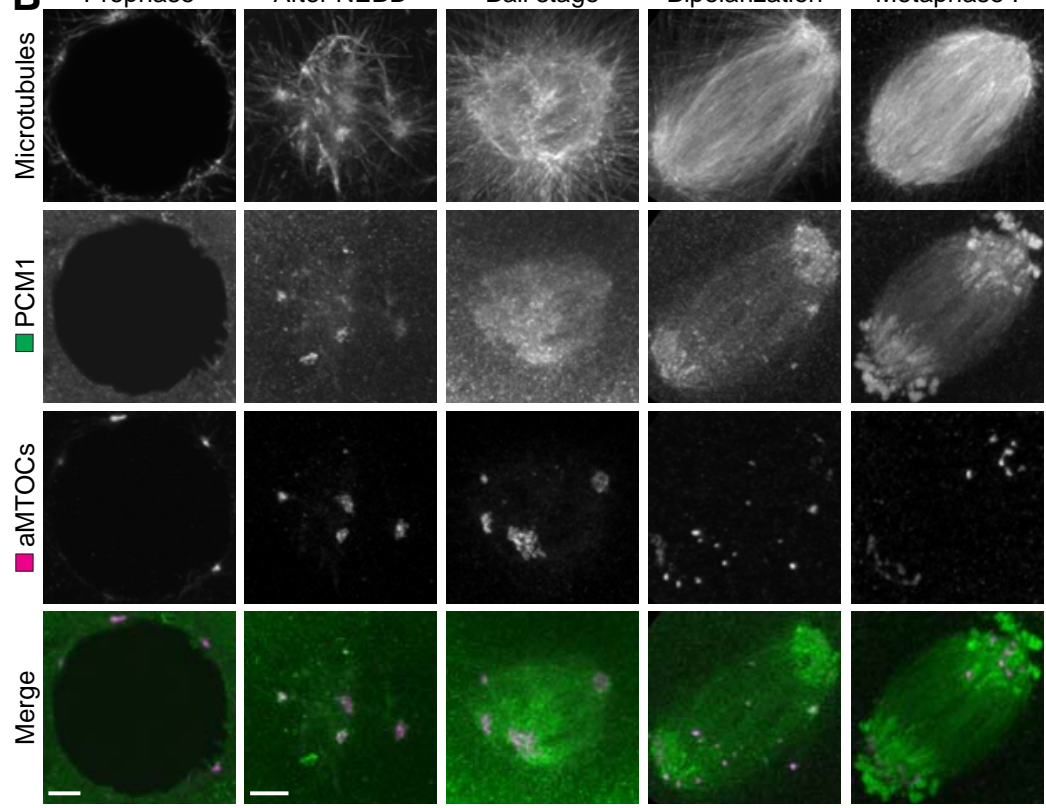

C Cold-treated metaphase I spindle

Merge
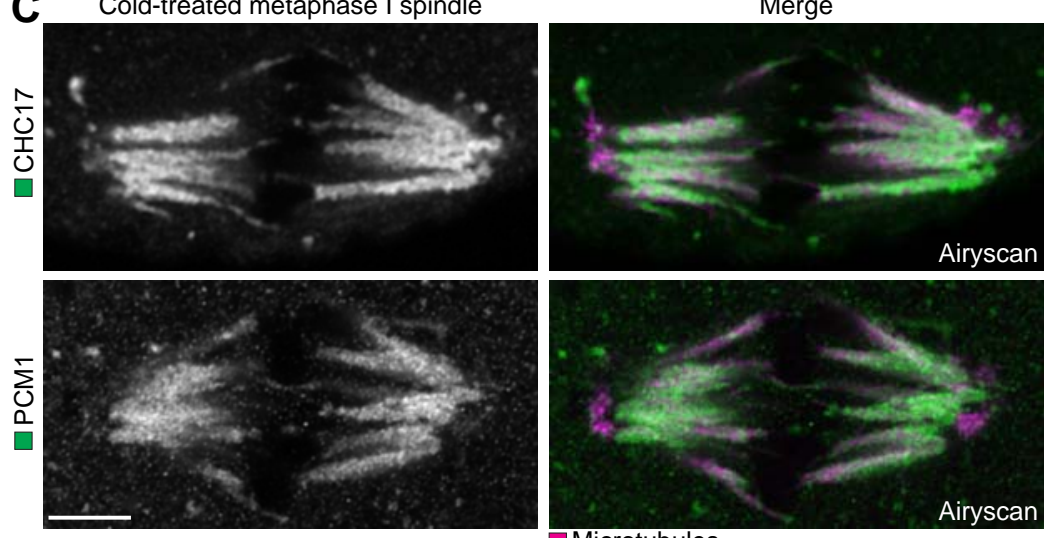

Microtubules 
Fig. S11 CHC17 and PCM1 have a similar localization pattern as TACC3 throughout oocyte maturation. (A) Immunofluorescence images of $\mathrm{CHC} 17$ in mouse oocytes fixed at different times after NEBD. Gray, microtubules ( $\alpha$-tubulin). Green, CHC17; magenta, aMTOCs (pericentrin). (B) Immunofluorescence images of PCM1 in mouse oocytes fixed at different times after NEBD. Gray, microtubules ( $\alpha$-tubulin). Green, PCM1; magenta, aMTOCs (pericentrin). Note that two different PCM1 antibodies were used for staining oocytes before and after NEBD. (C) Single confocal sections of cold-treated mouse metaphase I spindle. Green, CHC17 or PCM1; magenta, microtubules ( $\alpha$-tubulin). Scale bars, $5 \mu \mathrm{m}$. 
Figure S12

Acute MLN8237 addition
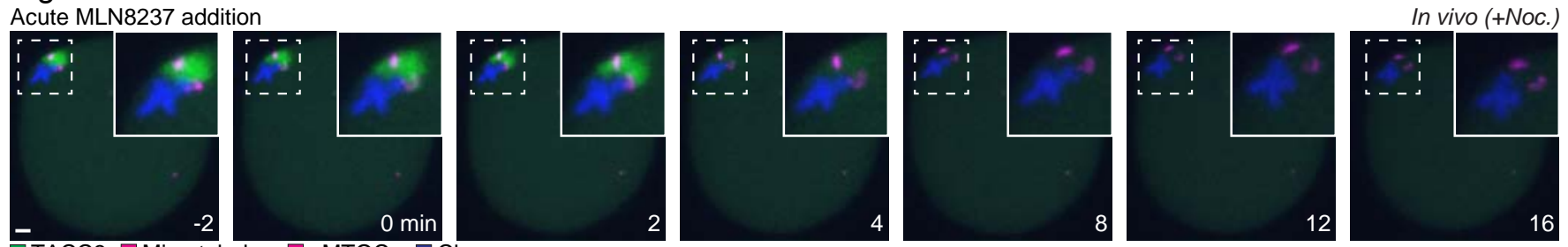

$\square$ TACC3 $\square$ Microtubules $\square$ aMTOCs $\square$ Chromosomes 
Fig. S12 Spherical condensates in acutely nocodazole-treated mouse metaphase I oocytes are dependent on AURA. Still images from time-lapse movies of spherical condensates in acutely MLN8237-treated mouse metaphase I oocytes pre-treated with $10 \mu \mathrm{M}$ nocodazole. Green, TACC3; magenta, microtubules (3×CyOFP-MAP4-MTBD) and aMTOCs (CEP192-mScarlet); blue, chromosomes (Hoechst). Insets are magnifications of regions outlined by dashed line boxes. Scale bar, $5 \mu \mathrm{m}$. Time is given as minutes after $500 \mathrm{nM}$ MLN8237 addition. 
Figure S13

A

B

Randomly oriented metaphase I

spindle in resin

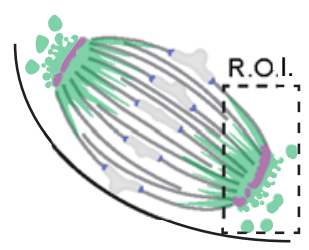

$\downarrow$

FIB-SEM

imaging at

$5 \mathrm{~nm}$ isotropic

voxels

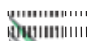

|บำ|IIIIIIIII

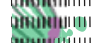

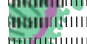

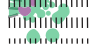

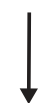

Rotation

and reslicing

of the

z-stack

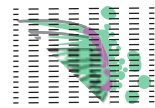

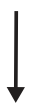

Semi-automatic threshold-based segmentation
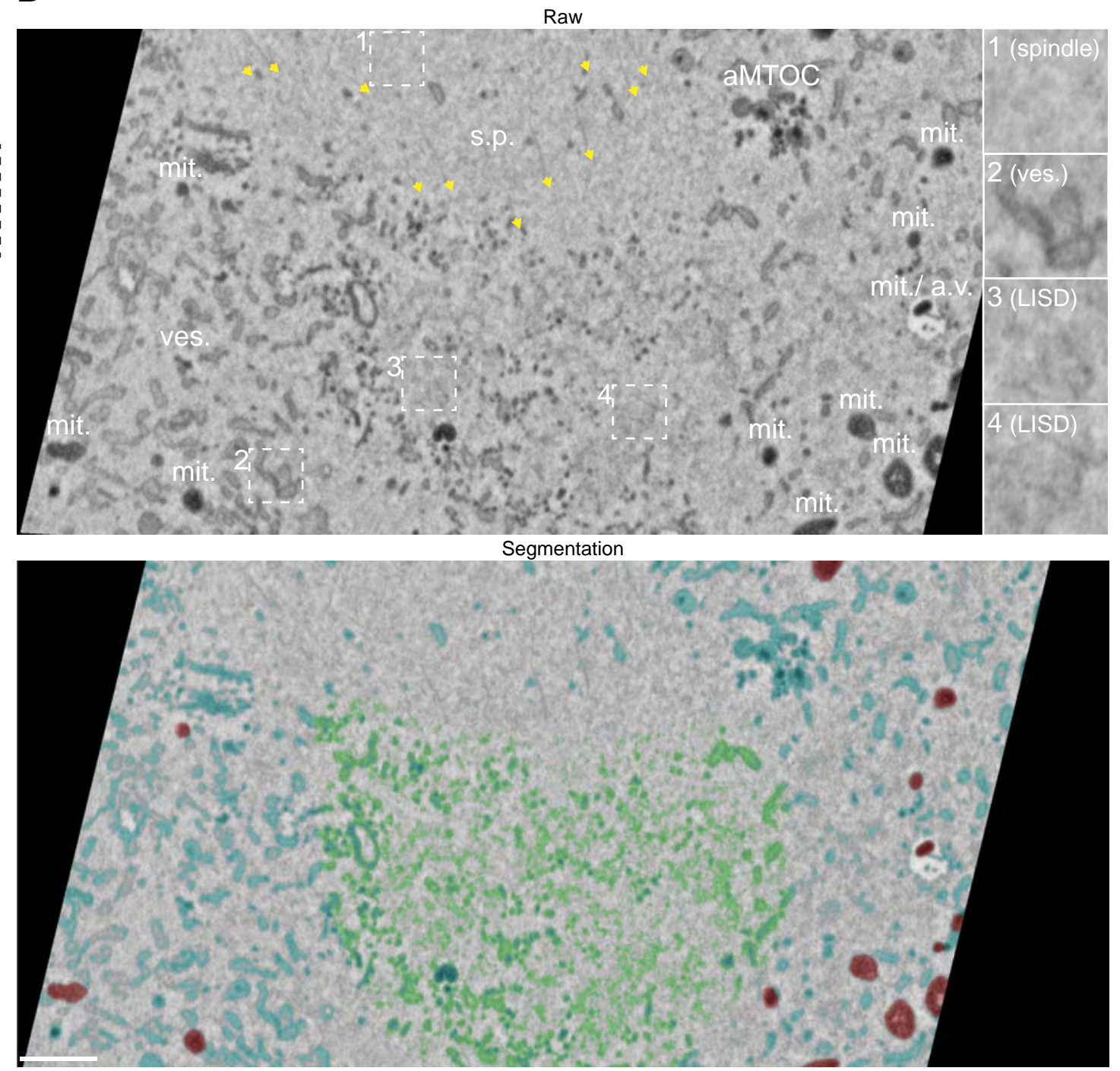

C
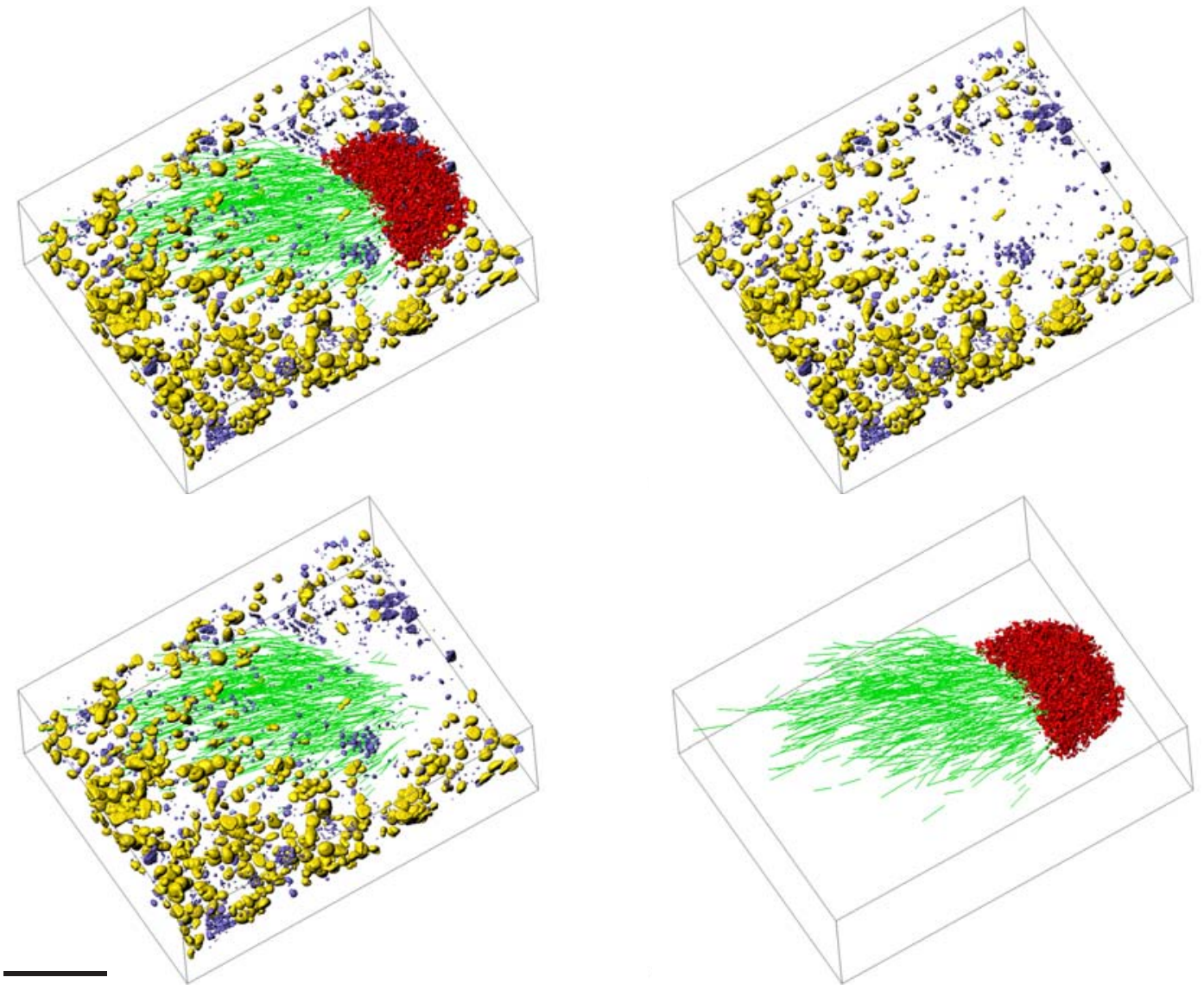

$\square$ Microtubules 口 LISD

$\square$ Mitochondria

口Membranes 
Fig. S13 The LISD is not enclosed by membranes. (A) A schematic representation showing the workflow for FIB-SEM of a spindle pole in mouse late metaphase I oocytes. (B) A single FIBSEM section showing the threshold-based segmentation. Yellow arrows indicate the running direction of microtubules. a.v., autophagic vacuole, mit., mitochondria, s.p., spindle pole and ves., vesicular structure. Note that the contrast within the spindle region only allowed for segmentation of microtubules. Insets are magnifications of regions outlined by dashed line boxes. Scale bar, 1 $\mu \mathrm{m}$. (C) 3D rendered model of a spindle pole in mouse late metaphase I oocytes. Green, microtubules; red, LISD; yellow, mitochondria; purple, membranes. Scale bar, $5 \mu \mathrm{m}$. 
Figure S14

A

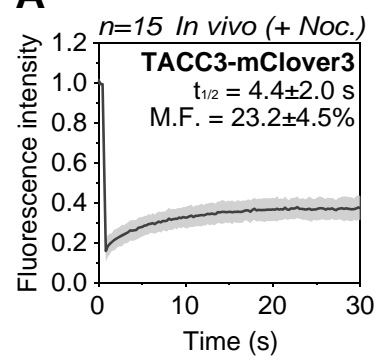

B
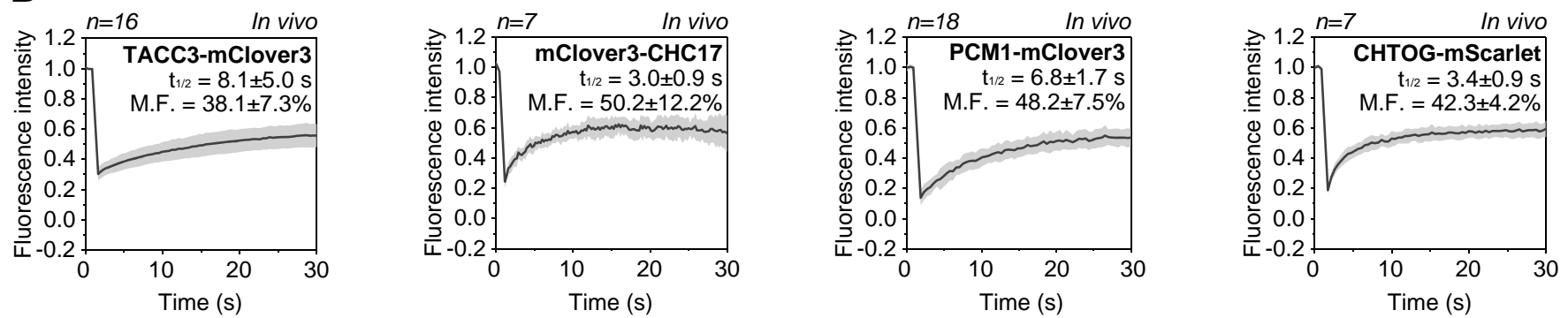

\section{C}
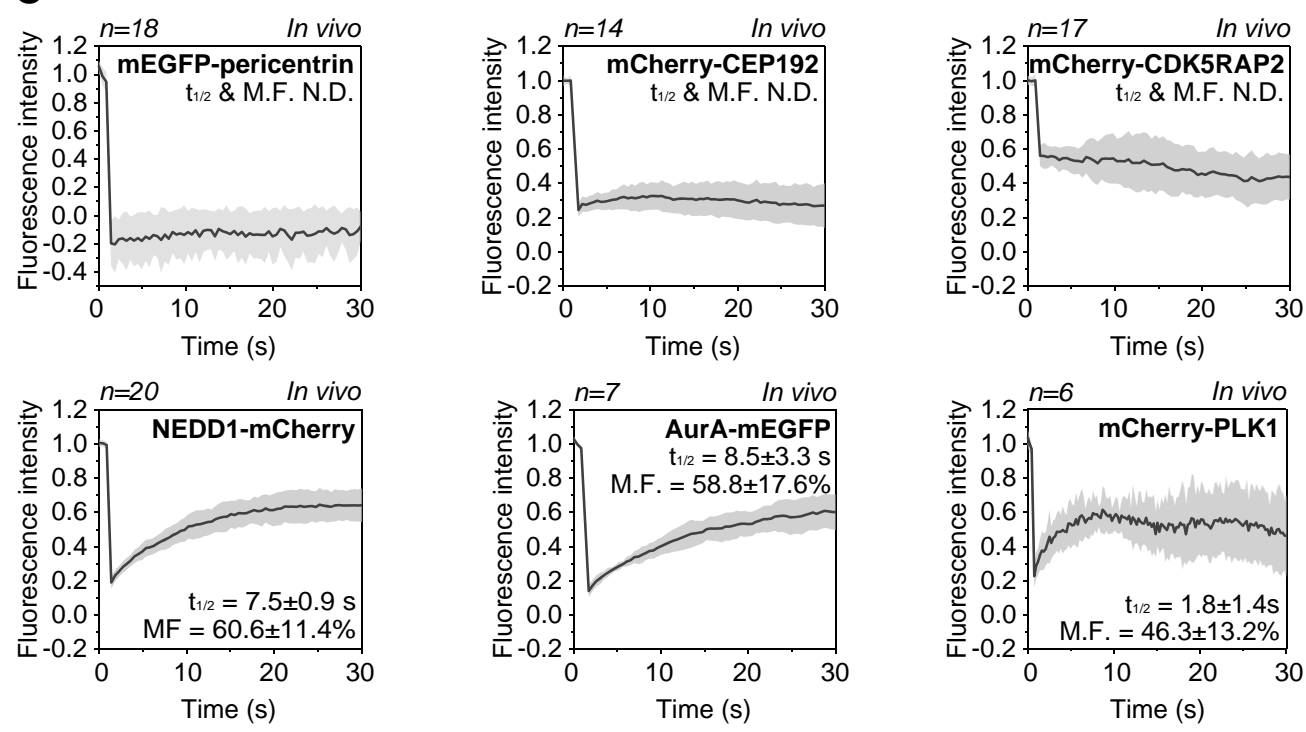
Fig. S14 Proteins in the LISD and spherical condensates are dynamic. (A) FRAP of TACC3mClover3 in spherical condensates in acutely $10 \mu \mathrm{M}$ nocodazole-treated mouse metaphase I oocytes. The number of analyzed spherical condensates is specified in italics. (B) FRAP of TACC3-mClover3, mClover3-CHC17, PCM1-mClover3 and chTOG-mScarlet in mouse metaphase I oocytes. The number of analyzed spindle poles is specified in italics. (C) FRAP of mEGFP-Pericentrin, mCherry-CEP192, mCherry-CDK5RAP2, NEDD1-mCherry, AURAmEGFP and mCherry-PLK1 in mouse metaphase I oocytes. The number of analyzed aMTOCs is specified in italics. M.F., mobile fraction. 
Figure S15

A
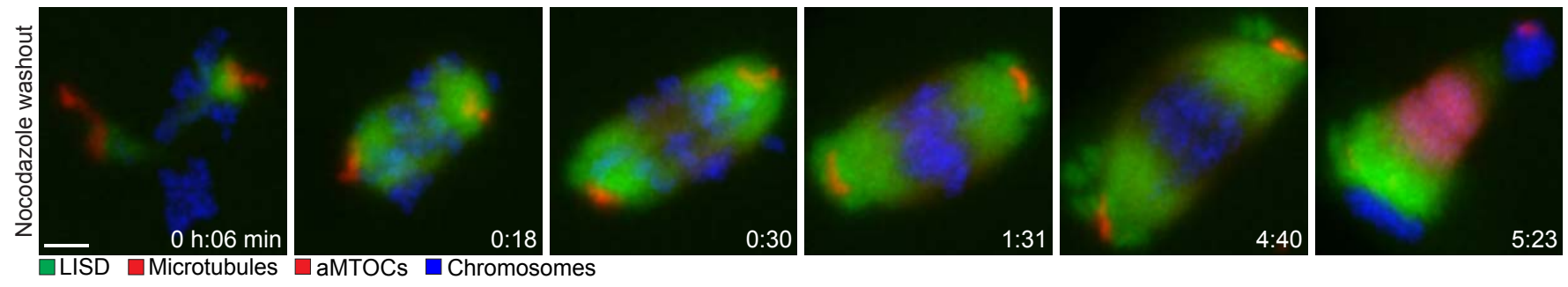

BAcute 1,6-hexanediol addition
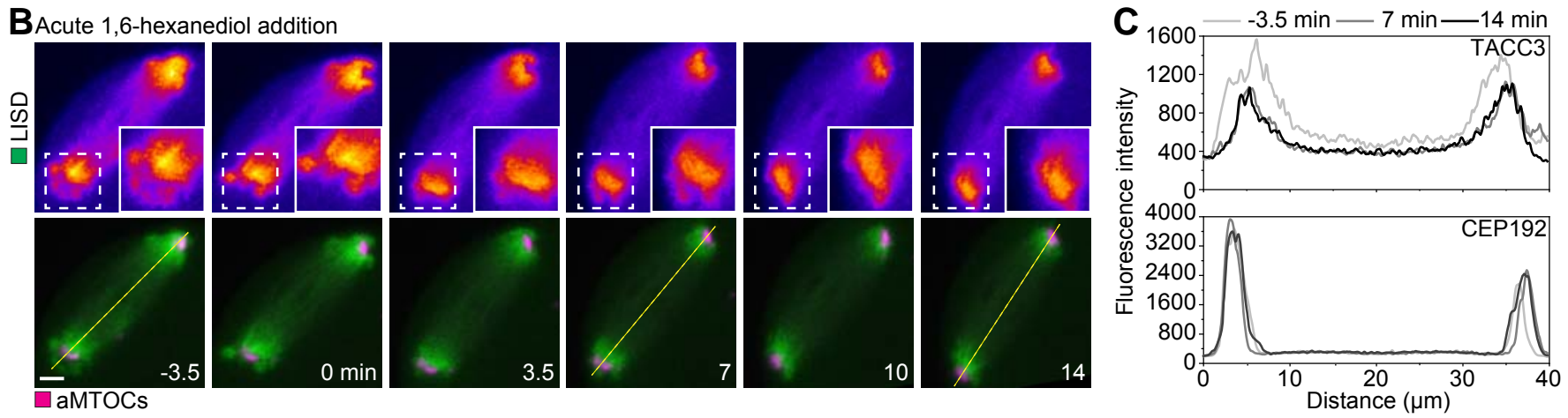

D

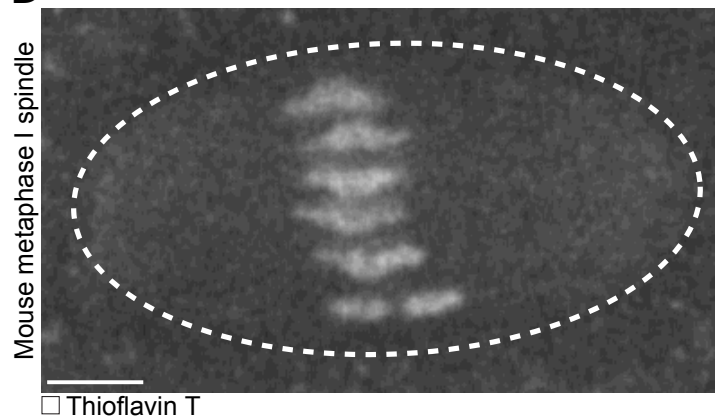


Fig. S15 The LISD is liquid-like. (A) Still images from time-lapse movies of mouse metaphase I oocytes after nocodazole washout. Green, LISD (TACC3-mClover3); red, microtubules (3×CyOFP-MAP4-MTBD) and aMTOCs (CEP192-mScarlet); blue, chromosomes (H2B-miRFP). (B) Still images from time-lapse movies of acutely 1,6-hexanediol-treated mouse metaphase I oocytes. Green, LISD (TACC3-mClover3); magenta, aMTOCs (CEP192-mScarlet). Insets are magnifications of regions outlined by dashed line boxes. Time is given as minutes after 3.5\% 1,6hexanediol addition. Yellow lines mark positions corresponding to fluorescence profiles in (C). (C) Quantification of fluorescence intensity of TACC3-mClover3 and CEP192-mScarlet in (A) at different times. (D) Fluorescence image of thioflavin T-stained mouse metaphase I oocytes. The spindle is outlined in white. Scale bars, $5 \mu \mathrm{m}$. 
Figure S16
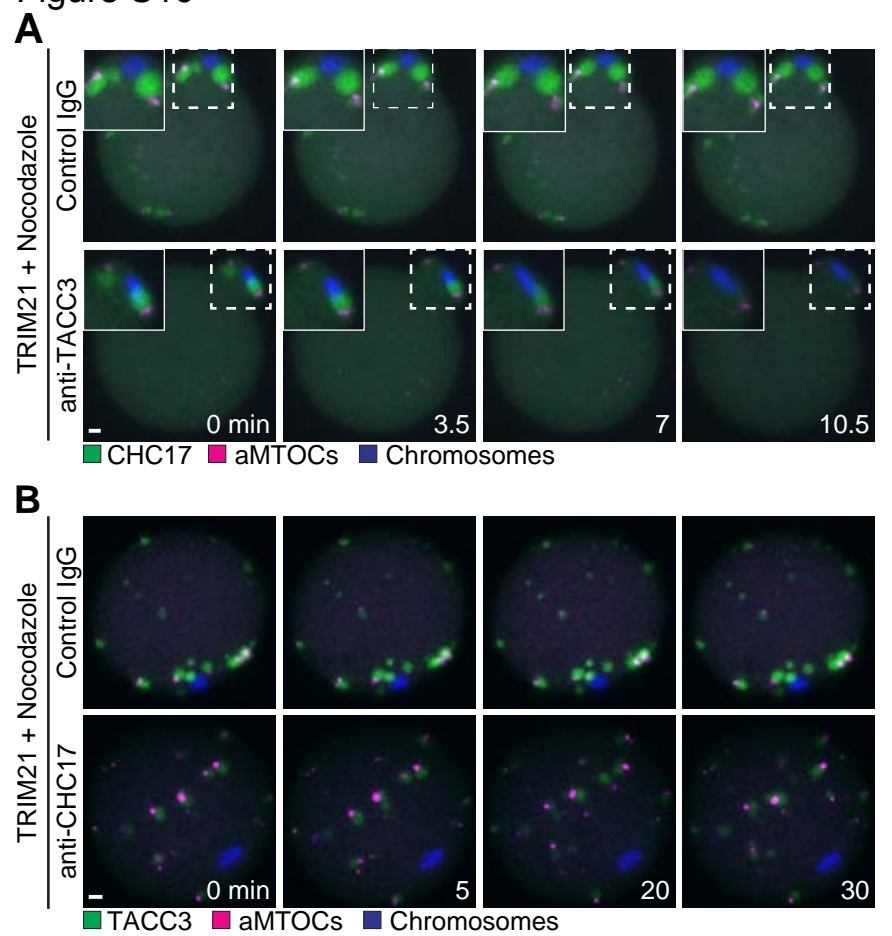
Fig. S16 Spherical condensates in acutely nocodazole-treated mouse metaphase I oocytes are disassembled by TACC3 depletion, but mildly affected by CHC17 depletion. (A) Still images from time-lapse movies of control and TACC3-depleted mouse metaphase I oocytes pre-treated with $10 \mu \mathrm{M}$ nocodazole. Green, CHC17; magenta, aMTOCs (CEP192-mScarlet); blue, chromosomes (H2B-miRFP). (B) Still images from time-lapse movies of control and CHC17depleted mouse metaphase I oocytes pre-treated with $10 \mu \mathrm{M}$ nocodazole. Green, TACC3; magenta, aMTOCs (CEP192-mScarlet); blue, chromosomes (H2B-miRFP). Scale bars, $5 \mu \mathrm{m}$. 


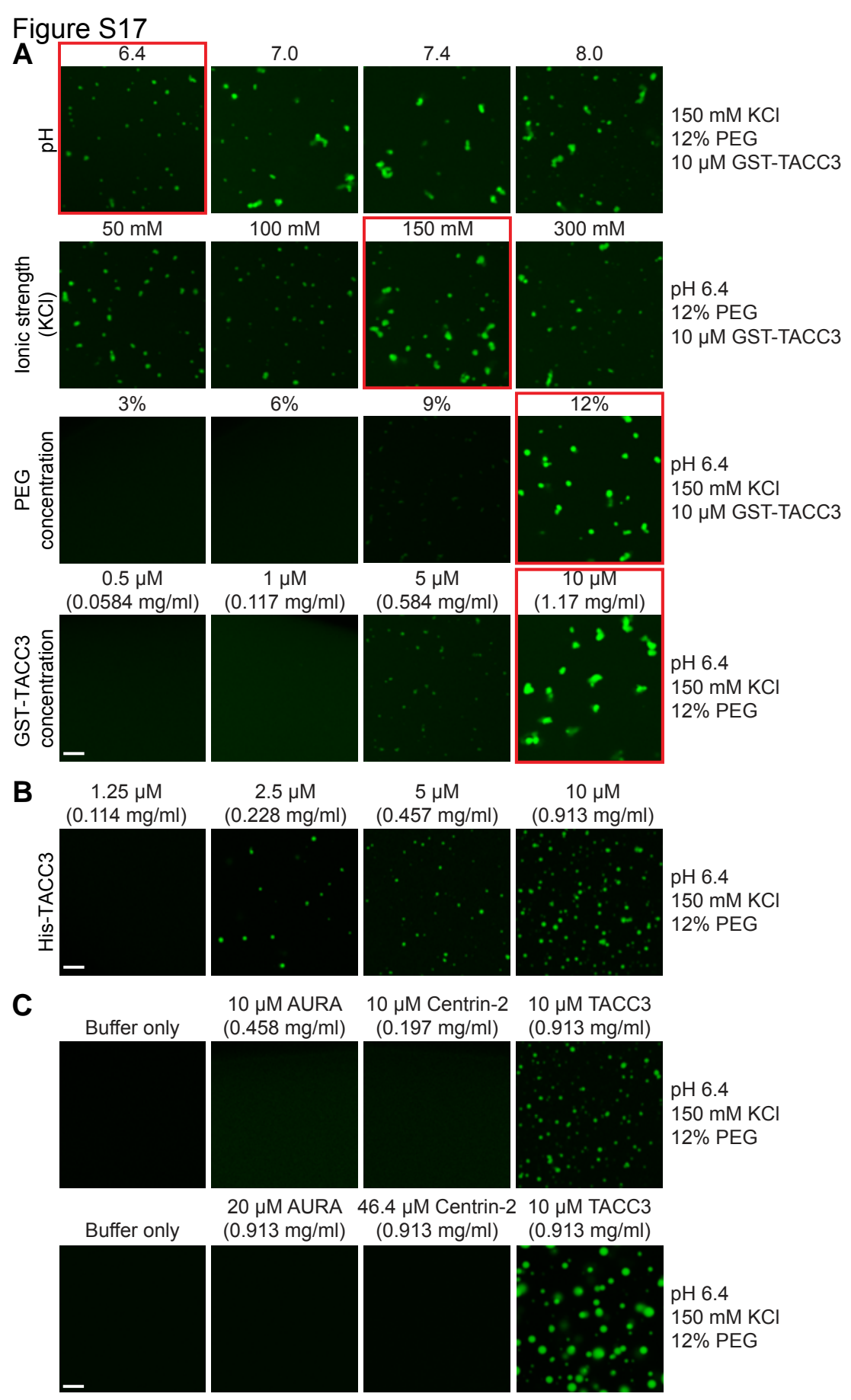

\section{D}

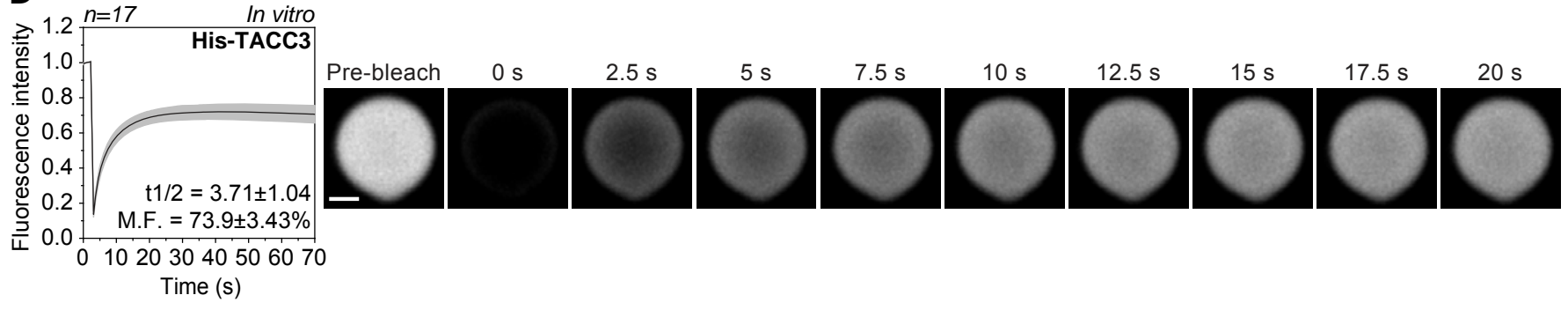


Fig. S17 TACC3 phase-separates in vitro. (A) Fluorescence images of GST-TACC3 droplets at different $\mathrm{pH}$ values, ionic strengths, PEG concentrations and protein concentrations. Green, GSTTACC3. (B) Fluorescence images of His-TACC3 droplets in pH 6.4 buffer with $150 \mathrm{mM} \mathrm{KCl}$ and 12\% PEG. Green, His-TACC3. (C) Fluorescence images of AURA, Centrin-2 and His-TACC3 in pH 6.4 buffer with $150 \mathrm{mM} \mathrm{KCl}$ and 12\% PEG. (D) FRAP of His-TACC3 droplets in pH 6.4 buffer with $150 \mathrm{mM} \mathrm{KCl}$ and 12\% PEG. Gray, His-TACC3. The number of analyzed droplets is specified in italics. M.F., mobile fraction. Scale bar, $1 \mu \mathrm{m}$. Scale bars, $5 \mu \mathrm{m}$ unless otherwise specified. 
Figure S18

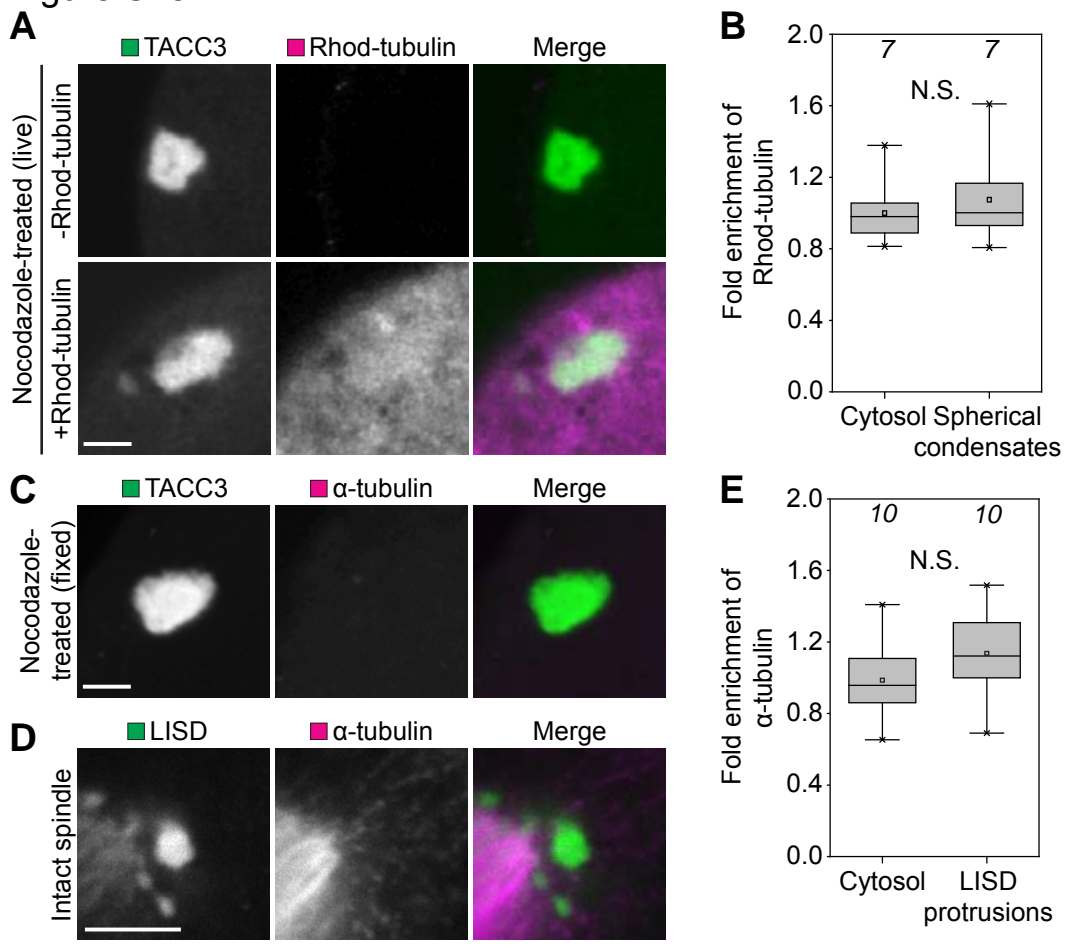


Fig. S18 LISD protrusions and spherical condensates do not concentrate tubulin dimers. (A) Fluorescence images of non-injected and rhodamine (Rhod)-tubulin-injected mouse metaphase I oocytes pre-treated with $10 \mu \mathrm{M}$ nocodazole. Green, TACC3; magenta, Rhod-tubulin. (B) Quantification of fold enrichment of Rhod-tubulin in the cytosol and in the spherical condensates. The number of analyzed oocytes is specified in italics. (C) Immunofluorescence images of spherical condensates in acutely $10 \mu \mathrm{M}$ nocodazole-treated mouse metaphase I oocytes. Green, TACC3; magenta, $\alpha$-tubulin. (D) Immunofluorescence images of LISD protrusions in mouse metaphase I oocytes. Green, LISD (TACC3); magenta, $\alpha$-tubulin. (E) Quantification of fold enrichment of $\alpha$-tubulin in the cytosol and in the LISD protrusions. The number of analyzed oocytes is specified in italics. Scale bars, $5 \mu \mathrm{m}$. 
Figure S19
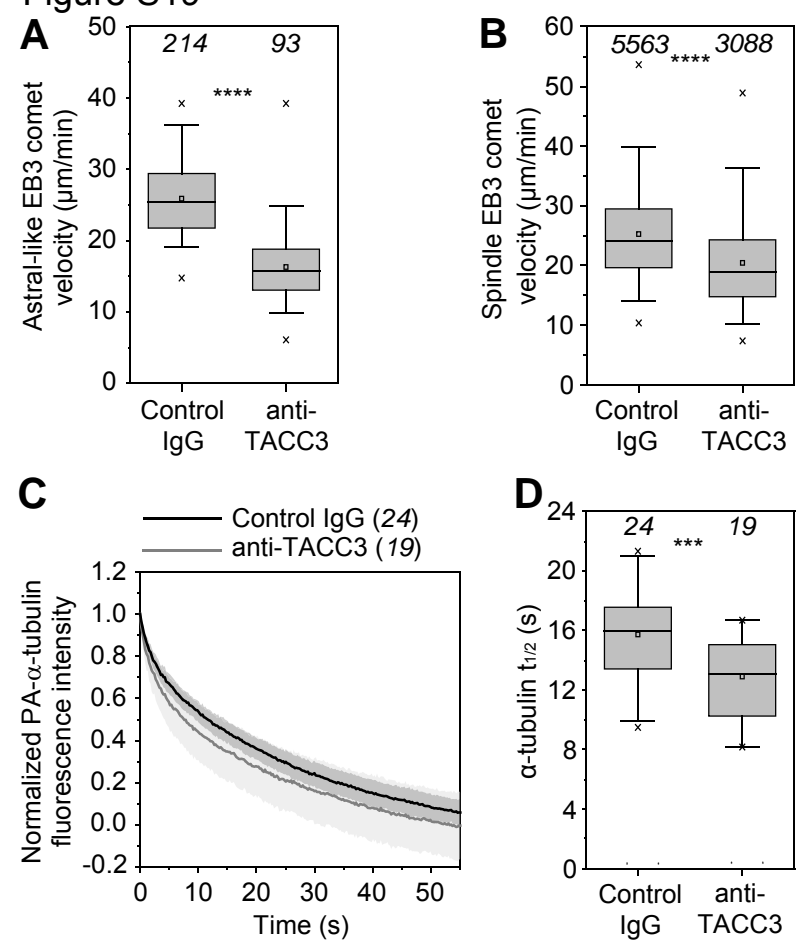
Fig. S19 Destabilized microtubules in TACC3-depleted oocytes. (A and B) Quantification of EB3 comet velocity of astral-like and spindle microtubules in control and TACC3-depleted mouse metaphase I oocytes. (C) Dissipation of mPA-GFP- $\alpha$-tubulin fluorescence signal in control and TACC3-depleted mouse metaphase I oocytes. (D) Distribution of half-life of mPA-GFP- $\alpha$-tubulin fluorescence signal dissipation in control and TACC3-depleted mouse metaphase I oocytes. The number of analyzed comets or spindle poles is specified in italics. Error bars (shaded areas) represent S.D. 
Figure S20
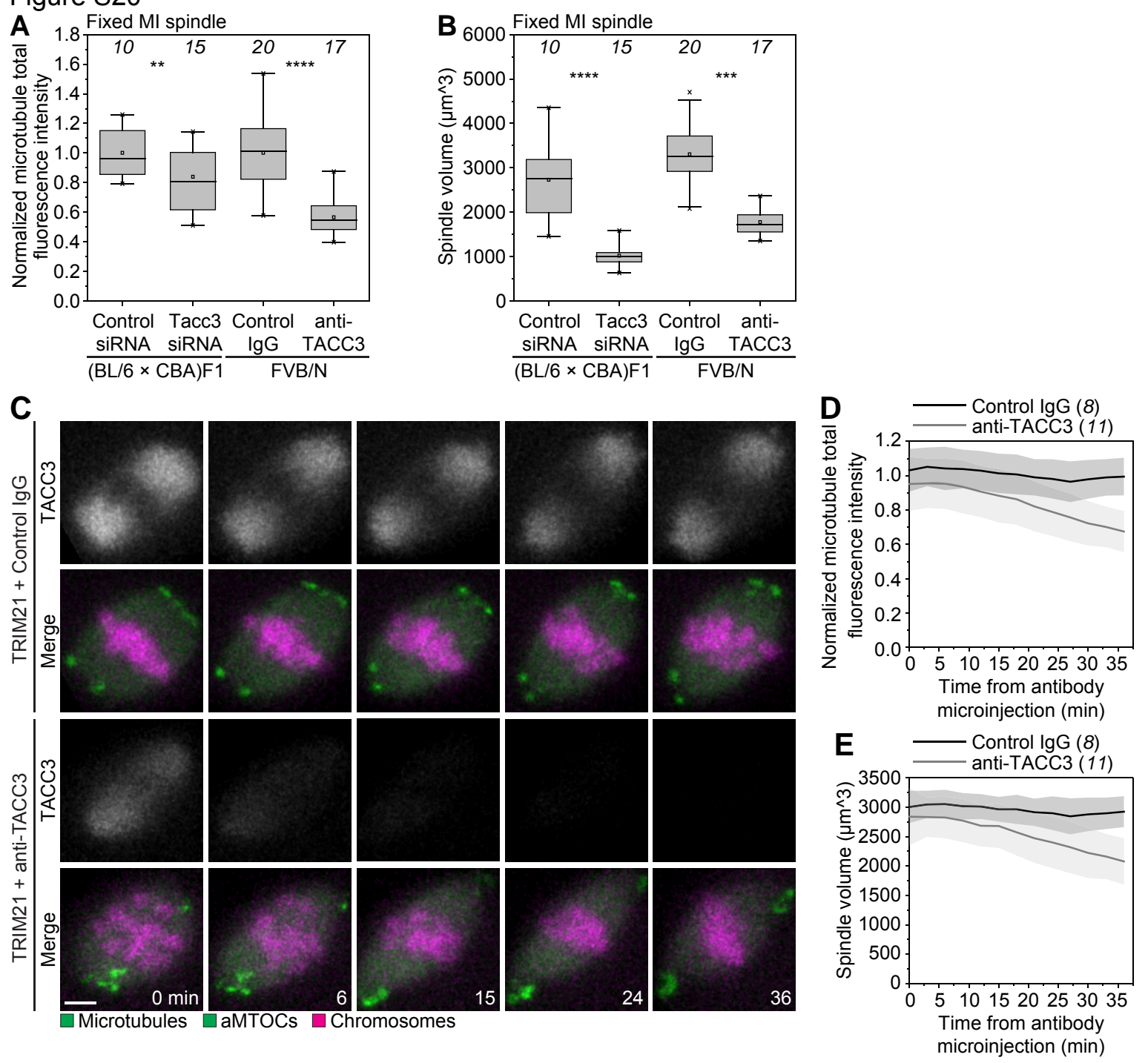
Fig. S20 RNAi and acute depletion of TACC3 also leads to spindle defects in mouse metaphase I oocytes. (A and B) Quantification of total fluorescence intensity of microtubules and spindle volume in control siRNA-injected, TACC3 siRNA-injected, control and TACC3-depleted mouse metaphase I oocytes. (C) Still images from time-lapse movies of control and acutely TACC3-depleted mouse metaphase I oocytes. Green, microtubules (3×CyOFP-MAP4-MTBD) and aMTOCs (CEP192-mScarlet). Magenta, chromosomes (H2B-miRFP). Time is given as minutes after antibody microinjection. ( $\mathbf{D}$ and $\mathbf{E}$ ) Quantification of total fluorescence intensity of microtubules and spindle volume in control and acutely TACC3-depleted mouse metaphase I oocytes. The number of analyzed oocytes is specified in italics. Error bars (shaded areas) represent S.D. Scale bars, $5 \mu \mathrm{m}$. 
Figure S21

A

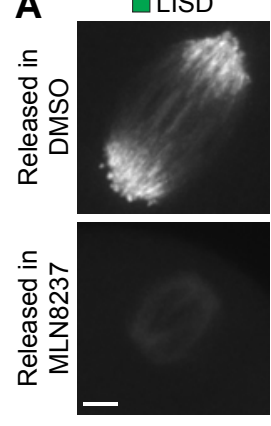

$\square$ aMTOCs

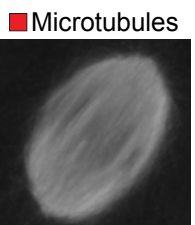

Merge
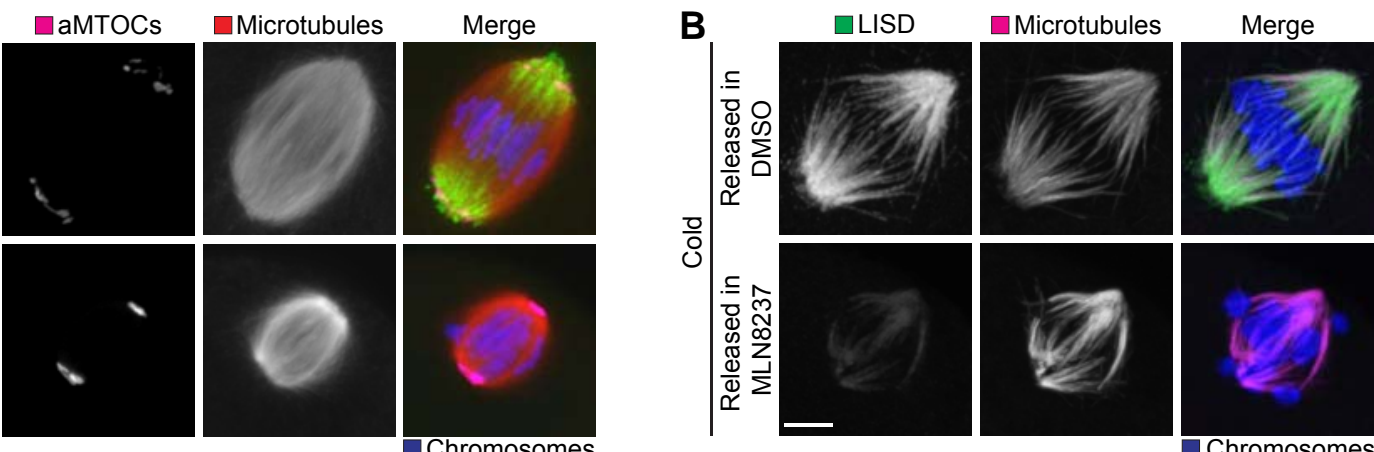

ป
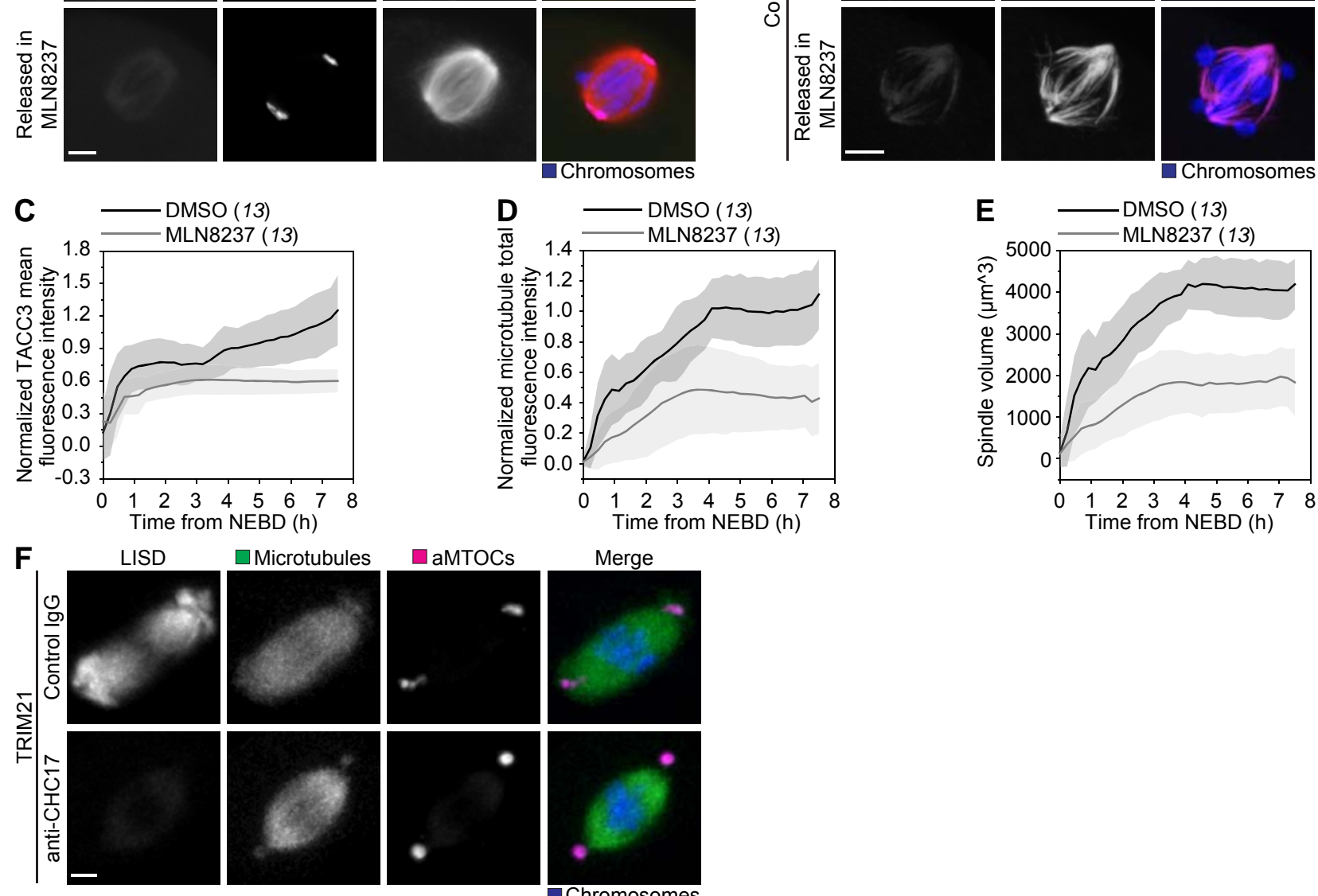

$$
\text { Merge }
$$
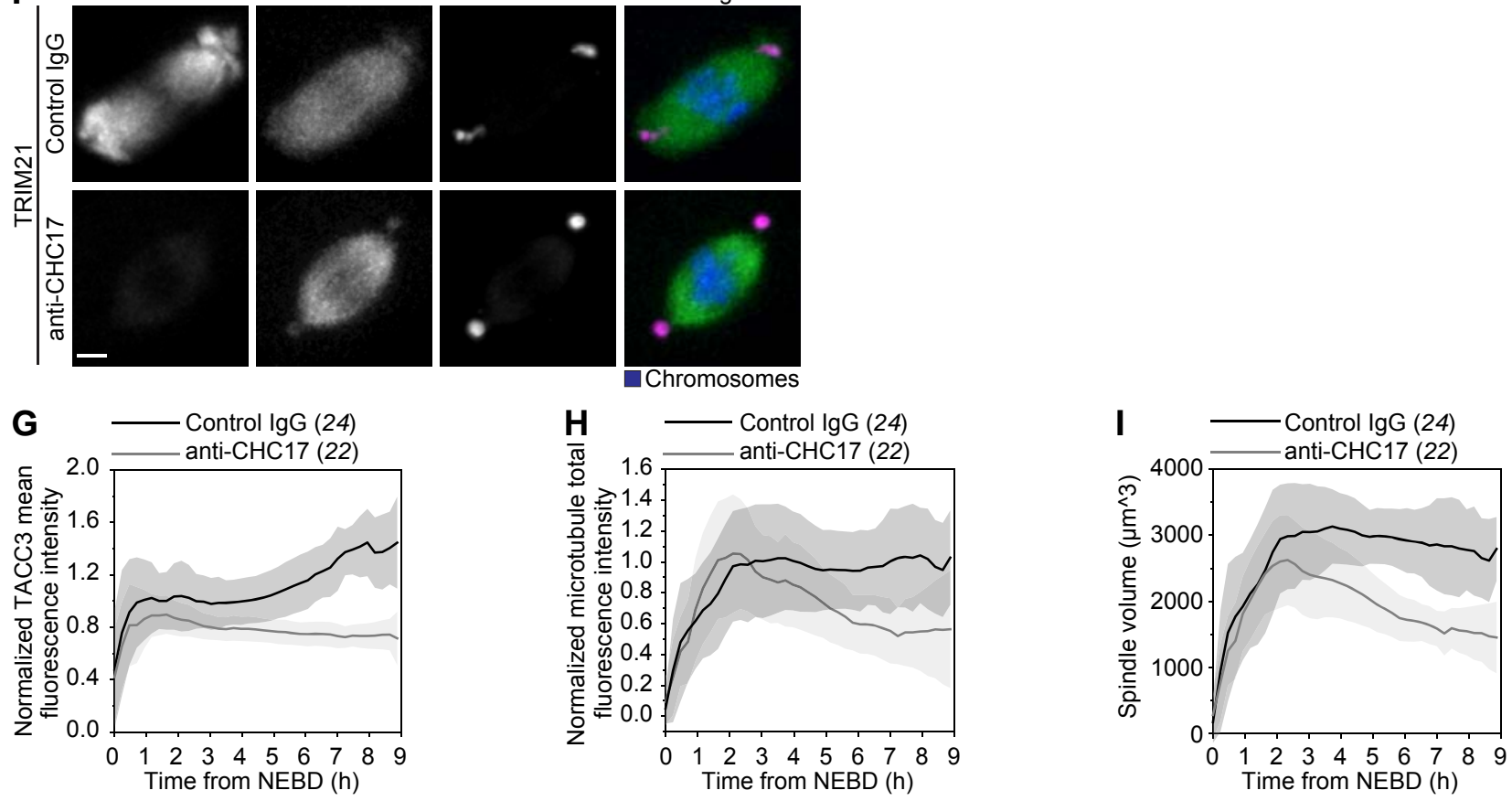
Fig. S21 Inhibition of AURA and depletion of CHC17 similarly leads to spindle defects in mouse metaphase I oocytes. (A) Immunofluorescence images of DMSO- and $500 \mathrm{nM}$ MLN8237treated mouse metaphase I oocytes. Green, LISD (TACC3); magenta, aMTOCs (Pericentrin); red, microtubules ( $\alpha$-tubulin); blue, chromosomes (Hoechst). (B) Immunofluorescence images of DMSO- and $500 \mathrm{nM}$ MLN8237-treated mouse metaphase I oocytes treated with cold. Green, LISD (TACC3); magenta, microtubules ( $\alpha$-tubulin); blue, chromosomes (Hoechst). (C) Quantification of mean fluorescence intensity of TACC3-mClover3 in DMSO- and $500 \mathrm{nM}$ MLN8237-treated mouse oocytes. (D and E) Quantification of total fluorescence intensity of microtubules and spindle volume in DMSO- and $500 \mathrm{nM}$ MLN8237-treated mouse oocytes. (F) Still images from time-lapse movies of control and CHC17-depleted mouse metaphase I oocytes. Gray, LISD (TACC3-mClover3); green, microtubules (3×CyOFP-MAP4-MTBD); magenta, aMTOCs (CEP192-mScarlet); blue, chromosomes (H2B-miRFP). (G) Quantification of mean fluorescence intensity of TACC3-mClover3 in control and CHC17-depleted mouse oocytes. (H and I) Quantification of total fluorescence intensity of microtubules and spindle volume in control and CHC17-depleted mouse oocytes. The number of analyzed oocytes is specified in italics. Error bars (shaded areas) represent S.D. Scale bars, $5 \mu \mathrm{m}$. 
Figure S22

A

B
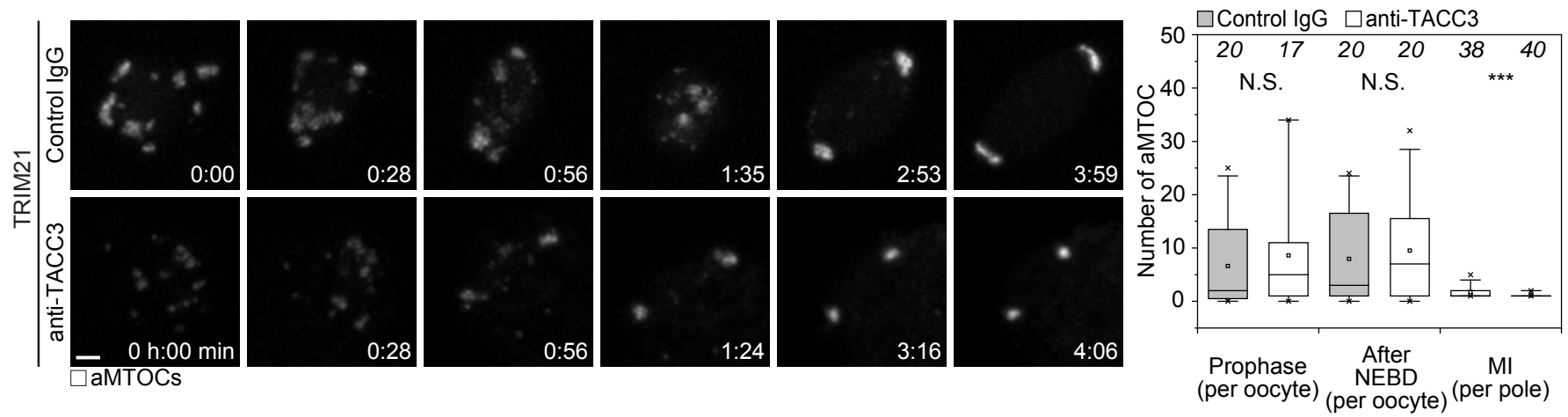

C

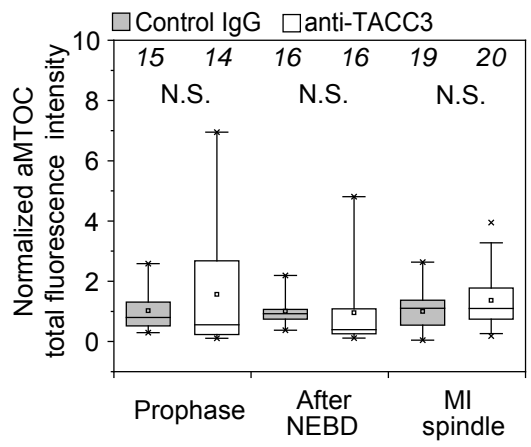

G

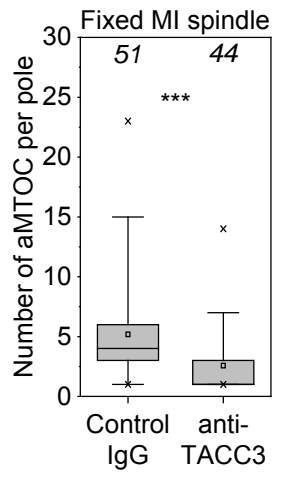

H
D
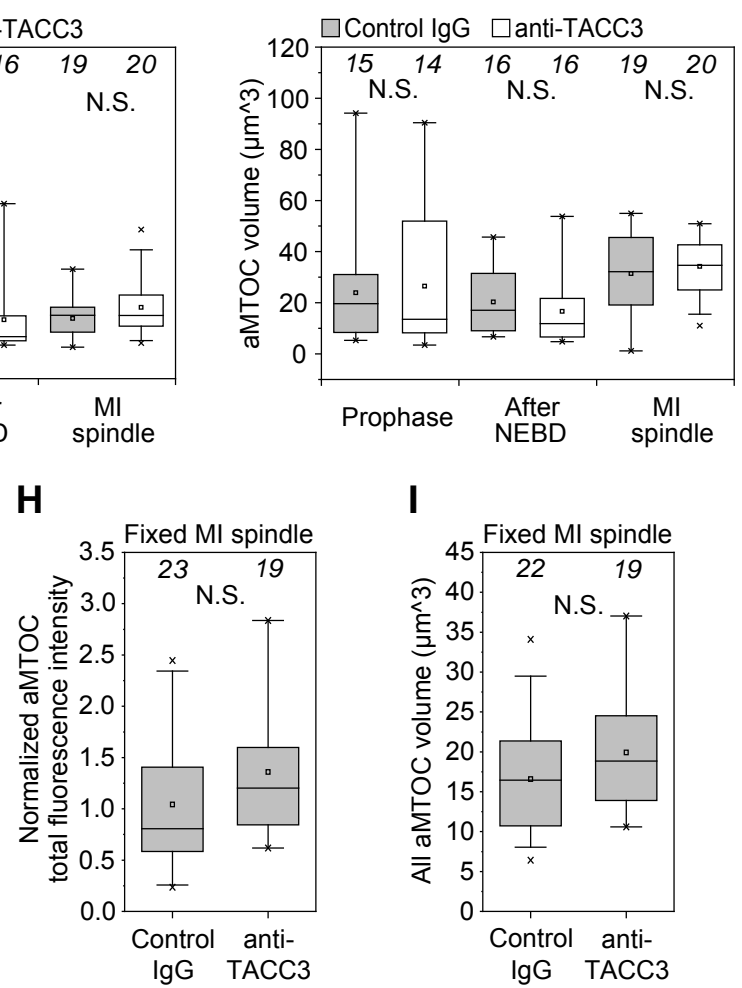

I

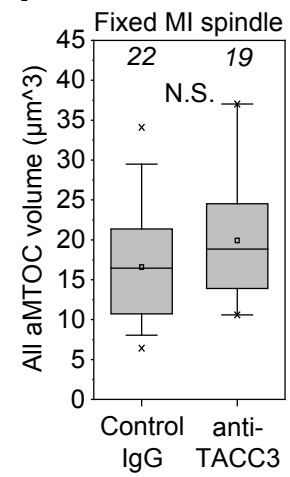

E

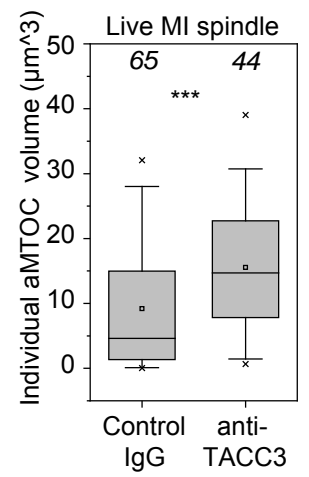

F

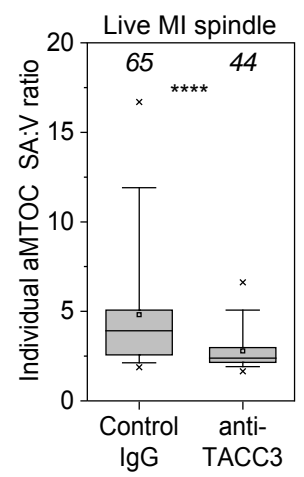

J

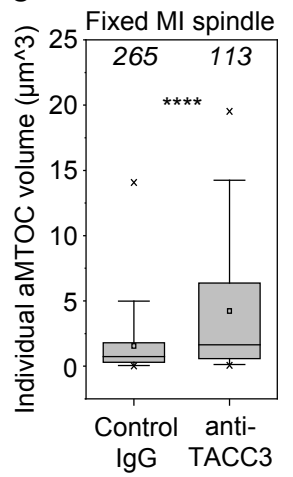

K

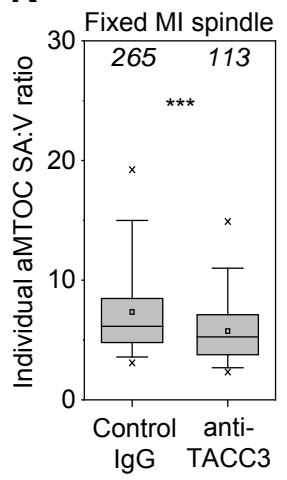


Fig. S22 Depletion of TACC3 leads to aMTOC defects in mouse metaphase I oocytes. (A) Still images from time-lapse movies of control and TACC3-depleted mouse metaphase I oocytes. Gray, aMTOCs (CEP192-mScarlet). Time is given as hours:minutes after NEBD. (B) Quantification of the number of aMTOCs at spindle poles in control and TACC3-depleted mouse oocytes. (C and D) Quantification of total fluorescence intensity and volume of all aMTOCs at spindle poles in control and TACC3-depleted mouse oocytes. (E and F) Quantification of the volume and surface area:volume $(\mathrm{SA}: \mathrm{V})$ ratio of individual aMTOCs at spindle poles in control and TACC3-depleted mouse metaphase I oocytes. (G) Quantification of the number of aMTOCs per pole in control and TACC3-depleted mouse metaphase I oocytes. (H and I) Quantification of total fluorescence intensity and volume of all aMTOCs at spindle poles in control and TACC3depleted mouse metaphase I oocytes. ( $\mathbf{J}$ and $\mathbf{K}$ ) Quantification of the volume and SA:V ratio of individual aMTOCs at spindle poles in control and TACC3-depleted mouse metaphase I oocytes. The number of analyzed oocytes or aMTOCs is specified in italics. Scale bars, $5 \mu \mathrm{m}$. 
Figure S23

A Over-clustering of aMTOCs into two foci
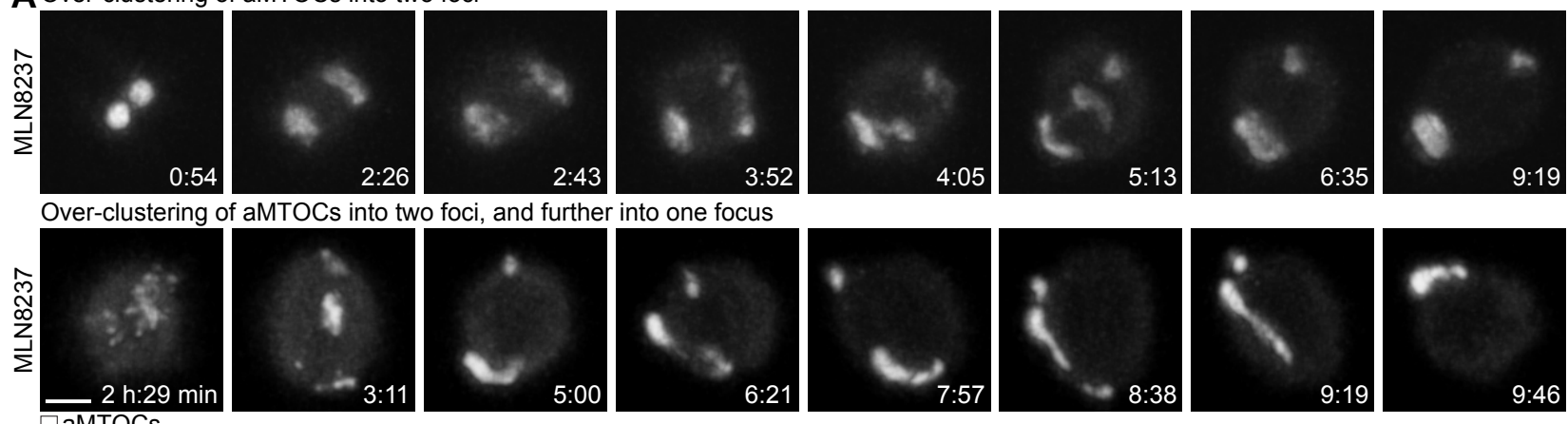

B

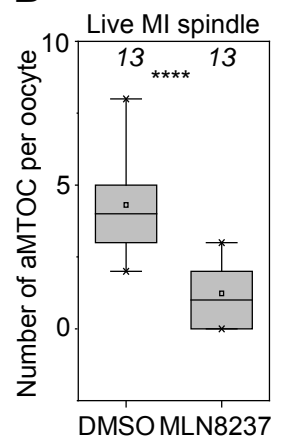

F

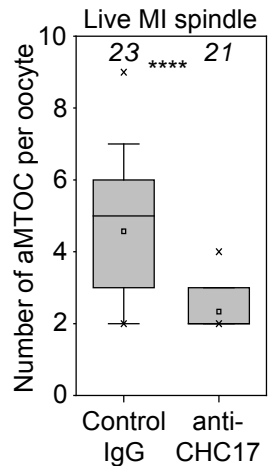

C

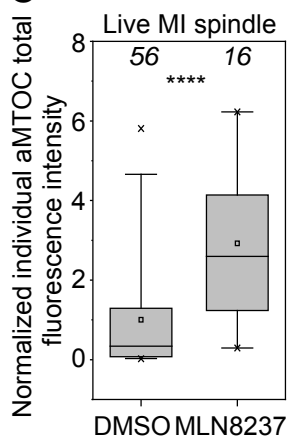

G

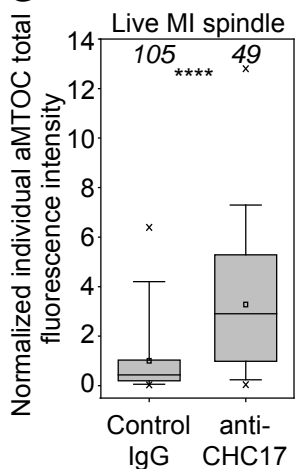

D

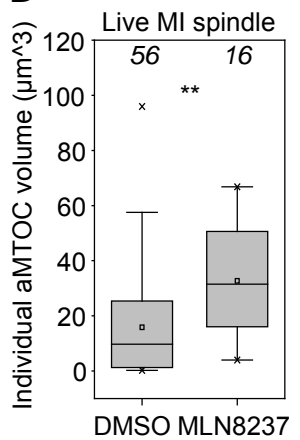

$\mathbf{H}$

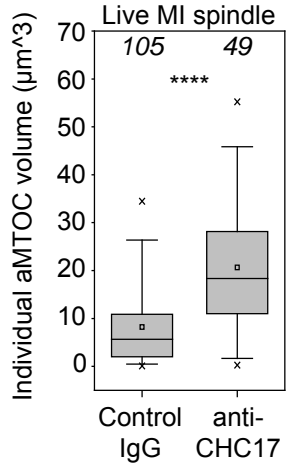

E

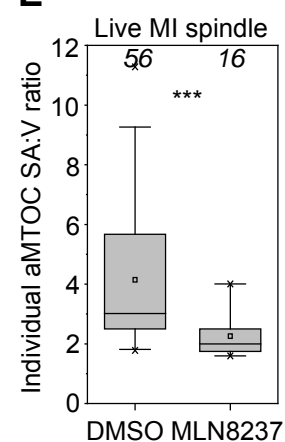

I

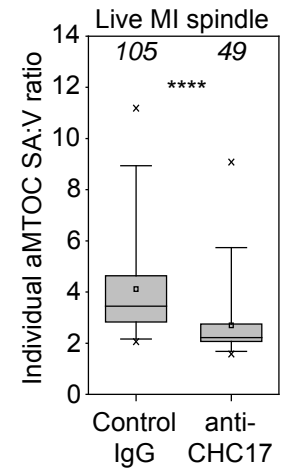


Fig. S23 Inhibition of AURA and depletion of CHC17 similarly leads to aMTOC defects in mouse metaphase I oocytes. (A) Still images from representative time-lapse movies of $500 \mathrm{nM}$ MLN8237-treated mouse oocytes. Gray, aMTOCs (CEP192-mScarlet). Time is given as hours:minutes after NEBD. (B) Quantification of the number of aMTOCs at spindle poles per oocyte in DMSO- and $500 \mathrm{nM}$ MLN8237-treated mouse metaphase I oocytes. (C, D and E) Quantification of total fluorescence intensity, volume and SA:V ratio of individual aMTOCs at spindle poles in DMSO- and $500 \mathrm{nM}$ MLN8237-treated mouse metaphase I oocytes. (F) Quantification of the number of aMTOC at spindle poles per oocyte in control and CHC17depleted mouse metaphase I oocytes. (G, H and I) Quantification of total fluorescence intensity, volume and SA:V ratio of individual aMTOCs at spindle poles in control and CHC17-depleted mouse metaphase I oocytes. The number of analyzed oocytes or aMTOCs is specified in italics. Scale bars, $5 \mu \mathrm{m}$. 
Figure S24

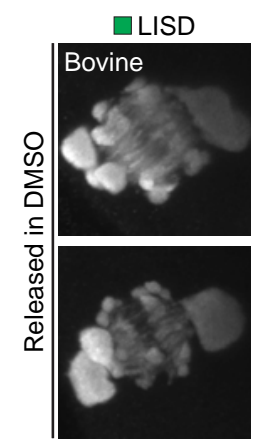

$\square$ Microtubules Merge
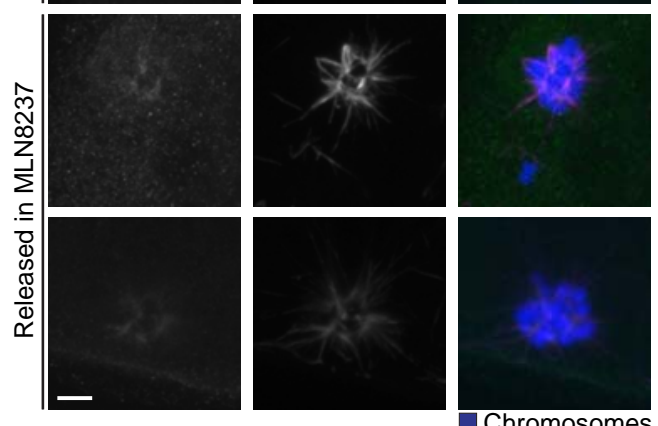
Fig. S24 Inhibition of AURA leads to severe spindle assembly defects in bovine metaphase I oocytes. Immunofluorescence images of DMSO- and $500 \mathrm{nM}$ MLN8237-treated bovine metaphase I oocytes. Green, LISD (TACC3); magenta, microtubules ( $\alpha$-tubulin); blue, chromosomes (Hoechst). Scale bar, $5 \mu \mathrm{m}$. 
Figure S25

A

$\square$ Control IgG $\square$ anti-TACC3

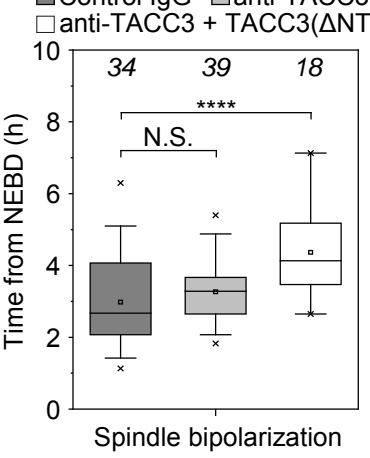

D

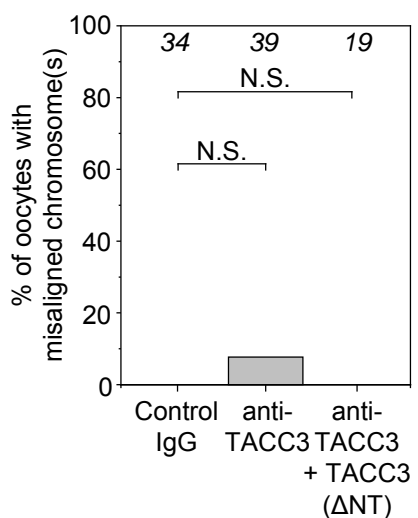

B

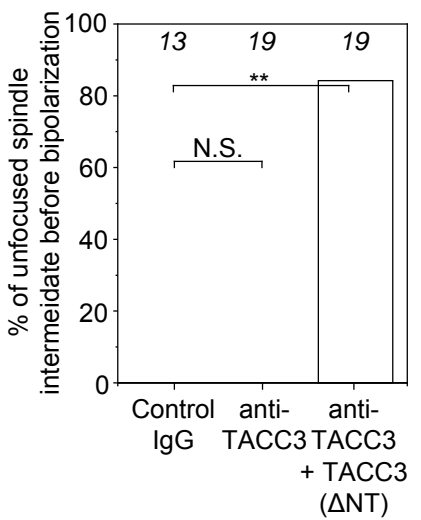

E

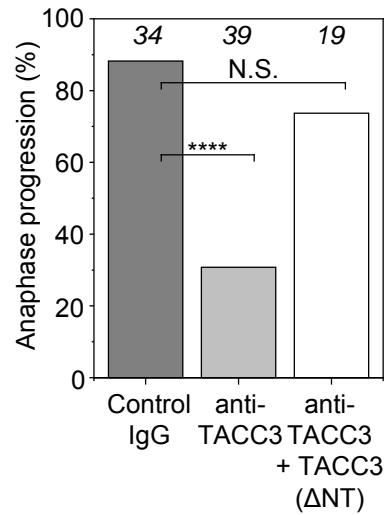

$\mathbf{F}$
C

$\square$ Normal aMTOCs $\square$ Over-clustered aMTOCs $\square$ Weakly associated aMTOCs

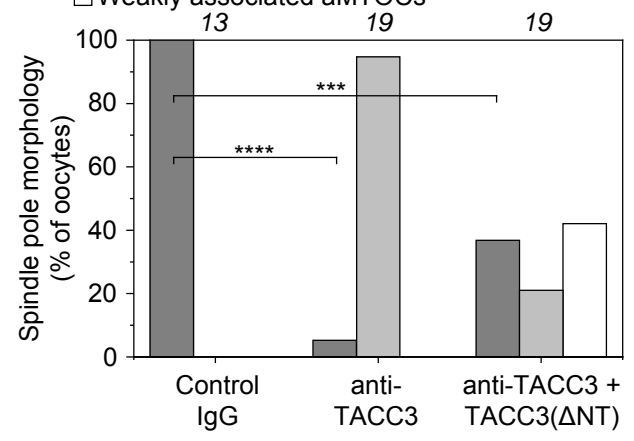

$\square$ Control IgG $\square$ anti-TACC3 $\square$ anti-TACC3 + TACC3( $\triangle N T)$

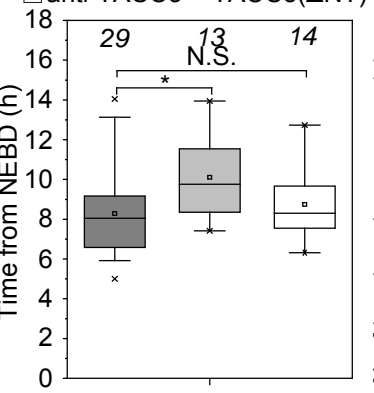

Anaphase onset
G

$\square 0$ lagging chromosome $\square \geq 1$ Midly lagging chromosome $\square \geq 1$ Severely lagging chromosome

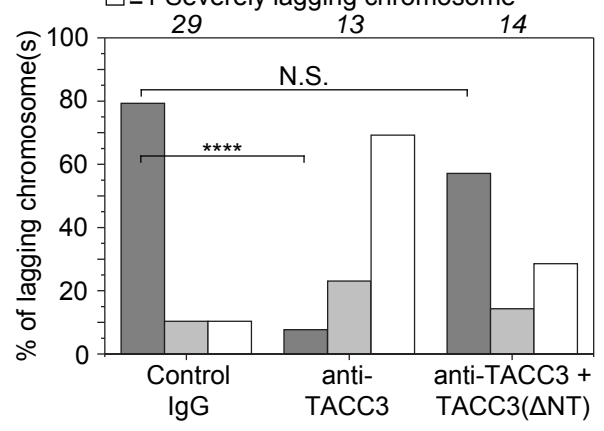


Fig. S25 TACC3( $\triangle \mathrm{NT})$ largely rescues chromosome segregation defects. (A) Quantification of the timing of spindle bipolarization in control, TACC3-depleted and TACC3( $\triangle \mathrm{NT})$-TACC3depleted mouse oocytes. (B) Quantification of unfocused spindle intermediates before bipolarization in control, TACC3-depleted and TACC3( $\triangle \mathrm{NT})$-TACC3-depleted mouse oocytes. (C) Quantification of spindle pole morphology in control, TACC3-depleted and TACC3( $\triangle \mathrm{NT})$ TACC3-depleted mouse oocytes. (D) Quantification of misaligned chromosomes in control, TACC3-depleted and TACC3( $\triangle \mathrm{NT})$-TACC3-depleted mouse oocytes. (E) Quantification of anaphase progression in control, TACC3-depleted and TACC3( $\triangle \mathrm{NT})$-TACC3-depleted mouse oocytes. (F) Quantification of the timing of anaphase onset in control, TACC3-depleted and TACC3 $(\triangle \mathrm{NT})$-TACC3-depleted mouse oocytes. (G) Quantification of lagging chromosomes in control, TACC3-depleted mouse and TACC3 $(\triangle N T)$-TACC3-depleted oocytes. The number of analyzed oocytes is specified in italics. Error bars (shaded areas) represent S.D. Scale bars, $5 \mu \mathrm{m}$. 
Figure S26

A
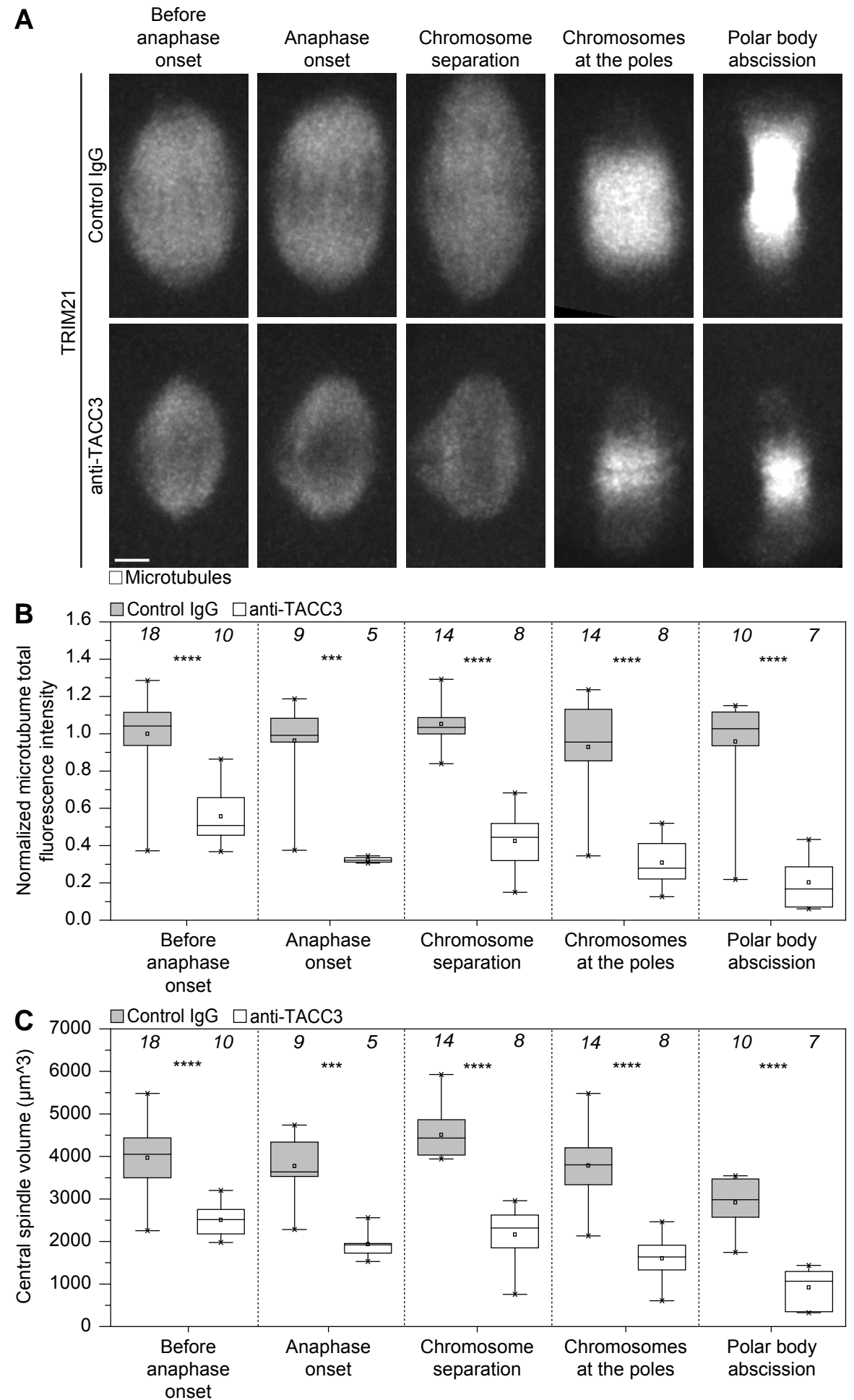
Fig. S26 The formation of the central spindle is affected in TACC3-depleted oocytes. (A) Still images from time-lapse movies of different stages of anaphase in control and TACC3-depleted mouse oocytes. Gray, microtubules (mClover3-MAP4-MTBD). Scale bar, $5 \mu \mathrm{m}$. (B and C) Quantification of total fluorescence intensity of microtubules and volume of the central spindle in control and TACC3-depleted mouse oocytes. The number of analyzed oocytes is specified in italics. 


\begin{tabular}{|c|c|c|c|c|c|c|}
\hline No. & Group & Protein & $\begin{array}{l}\text { Localization in mouse } \\
\text { metaphase I oocytes }\end{array}$ & Confirmed by & $\begin{array}{c}\text { Previously mapped } \\
\text { in mouse metaphase } \\
\text { I oocytes }\end{array}$ & $\begin{array}{c}\text { Antibodies and/or } \\
\text { constructs used in } \\
\text { other studies } \\
\end{array}$ \\
\hline 1 & \multirow[t]{15}{*}{$\begin{array}{l}\text { Centriolar } \\
\text { proteins }\end{array}$} & CENPJ/ CPAP & $\begin{array}{l}\text { No spindle localization (lost } \\
\text { from aMTOCs after NEBD) }\end{array}$ & Live imaging & 1 & $\checkmark(73)$ \\
\hline 2 & & CEP63 & No spindle localization & IF & I & $\begin{array}{c}\checkmark(\text { e.g. PMID } \\
27974163) \\
\end{array}$ \\
\hline 3 & & CEP135 & No spindle localization & IF & l & $\begin{array}{l}\checkmark(\text { e.g. PMID } \\
\text { 26609813) }\end{array}$ \\
\hline 4 & & CEP152 & $\begin{array}{l}\text { No spindle localization (lost } \\
\text { from aMTOCs after NEBD) }\end{array}$ & IF & 102 & $\begin{array}{l}\checkmark(\text { e.g. PMID } \\
28607478)\end{array}$ \\
\hline 5 & & CEP164 & No spindle localization & IF & / & $\begin{array}{l}\checkmark(\text { e.g. PMID } \\
29440264)\end{array}$ \\
\hline 6 & & CEP170 & LISD & IF & I & $x$ \\
\hline 7 & & CEP250/ CNAP1 & $\begin{array}{l}\text { aMTOCs (initially lost from } \\
\text { aMTOCs after NEBD) }\end{array}$ & IF, live imaging & 103 & $\begin{array}{c}\checkmark(\text { e.g. PMID } \\
27486244) / \checkmark(89)\end{array}$ \\
\hline 8 & & CETN2 & No spindle localization & IF, Live imaging & I & $\begin{array}{c}\checkmark(\text { e.g. PMID } \\
9004043) / \checkmark(70)\end{array}$ \\
\hline 9 & & Centrobin/ CNTROB & aMTOCs & Live imaging & 103 & $\checkmark(71)$ \\
\hline 10 & & CP110 & aMTOCs & IF & / & $\begin{array}{c}\checkmark(\text { e.g. PMID } \\
23486064)\end{array}$ \\
\hline 11 & & $\mathrm{CROCC} /$ rootletin & $\begin{array}{l}\text { No spindle localization (lost } \\
\text { from aMTOCs after NEBD) }\end{array}$ & Live imaging & l & $\checkmark(84)$ \\
\hline 12 & & LRRC45 & $\begin{array}{l}\text { No spindle localization (lost } \\
\text { from aMTOCs after NEBD) }\end{array}$ & Live imaging & I & $\sqrt{ }(76)$ \\
\hline 13 & & NEK2A & $\begin{array}{l}\text { No spindle localization (lost } \\
\text { from aMTOCs after NEBD) }\end{array}$ & Live imaging & I & $\checkmark(79)$ \\
\hline 14 & & Ninein & No spindle localization & IF & I & $\begin{array}{c}\checkmark(\text { e.g. PMID } \\
28290481)\end{array}$ \\
\hline 15 & & ODF2 & No spindle localization & IF & I & $\begin{array}{c}\checkmark(\text { e.g. PMID } \\
24813608) \\
\end{array}$ \\
\hline
\end{tabular}




\begin{tabular}{|c|c|c|c|c|c|c|}
\hline 16 & & SAS6 & $\begin{array}{l}\text { No spindle localization (lost } \\
\text { from aMTOCs after NEBD) }\end{array}$ & Live imaging & I & $\checkmark(85)$ \\
\hline 17 & & STIL & No spindle localization & IF & I & $\begin{array}{l}\checkmark(\text { e.g. PMID } \\
25342035)\end{array}$ \\
\hline 18 & \multirow{7}{*}{$\begin{array}{c}\text { Pericentriolar } \\
\text { material } \\
\text { proteins }\end{array}$} & AKAP9/ AKAP450 & LISD & IF, live imaging & / & $\begin{array}{c}\checkmark \text { (e.g. PMID } \\
28687497) / \checkmark \\
(62) \\
\end{array}$ \\
\hline 19 & & CEP120 & $\begin{array}{l}\text { aMTOCs, spindle } \\
\text { microtubules }\end{array}$ & IF & l & $x$ \\
\hline 20 & & CEP192 & aMTOCs & IF, live imaging & 102 & $x / \sqrt{x}(69)$ \\
\hline 21 & & CEP215/ CDK5RAP2 & aMTOCs & IF, live imaging & 104 & $\begin{array}{c}\checkmark \text { (e.g. PMID } \\
26297806) / \checkmark \\
(67)\end{array}$ \\
\hline 22 & & NEDD1 & $\begin{array}{c}\text { aMTOCs, spindle } \\
\text { microtubules }\end{array}$ & IF, live imaging & 105 & $\begin{array}{c}\checkmark \text { (e.g. 29547120) } \\
/ \checkmark(78) \\
\end{array}$ \\
\hline 23 & & $\mathrm{PCNT} /$ pericentrin & aMTOCs & IF, live imaging & 106 & $\begin{array}{c}\checkmark(\text { e.g. PMID } \\
22851319) / \checkmark(83)\end{array}$ \\
\hline 24 & & TUBG1/ $\gamma$-tubulin & $\begin{array}{l}\text { aMTOCs, spindle } \\
\text { microtubules }\end{array}$ & IF, live imaging & 107,108 & $\begin{array}{c}\checkmark \text { (e.g. PMID } \\
20890279) / \checkmark \\
(88)\end{array}$ \\
\hline 25 & \multirow{5}{*}{$\begin{array}{c}\text { Regulatory } \\
\text { kinases and } \\
\text { their } \\
\text { substrates }\end{array}$} & AURKA/ AURA & $\begin{array}{c}\text { aMTOCs, spindle } \\
\text { microtubules }\end{array}$ & IF, live imaging & 109 & $x / \checkmark(63)$ \\
\hline 26 & & pT288-AURA & aMTOCs & IF & 110 & $\begin{array}{c}\checkmark \text { (e.g. PMID } \\
29276128) \\
\end{array}$ \\
\hline 27 & & PLK1 & aMTOCs, kinetochores & IF, live imaging & 111 & $\begin{array}{c}\checkmark \text { (e.g. PMID } \\
25533956) / \checkmark \\
(89)\end{array}$ \\
\hline 28 & & pT210-PLK1 & aMTOCs, kinetochores & IF & 112,113 & $\begin{array}{c}\checkmark \text { (e.g. PMID } \\
26293378) \\
\end{array}$ \\
\hline 29 & & PLK4 & aMTOCs & $\mathrm{IF}$ & 5 & $\begin{array}{c}\checkmark \text { (e.g. PMID } \\
18056432) \\
\end{array}$ \\
\hline
\end{tabular}




\begin{tabular}{|c|c|c|c|c|c|c|}
\hline 30 & & pS395-NuMA & aMTOCs & IF & l & $\begin{array}{c}\text { (e.g. PMID } \\
28302921)\end{array}$ \\
\hline 31 & & pT2055-NuMA & aMTOCs & IF & I & $x$ \\
\hline 32 & \multirow{3}{*}{$\begin{array}{c}\text { Other } \\
\text { centrosomal } \\
\text { proteins }\end{array}$} & DISC1 & aMTOCs & IF & I & $\begin{array}{c}\checkmark \text { (e.g. PMID } \\
24560582)\end{array}$ \\
\hline 33 & & KIZ & LISD & IF & I & $x$ \\
\hline 34 & & TOP2A & aMTOCs, kinetochores & IF & I & $\begin{array}{c}\checkmark \text { (e.g. PMID } \\
25762097)\end{array}$ \\
\hline 35 & \multirow{7}{*}{$\begin{array}{l}\text { Centriolar } \\
\text { satellite } \\
\text { proteins }\end{array}$} & BBS4 & No spindle localization & Live imaging & I & $\checkmark(64)$ \\
\hline 36 & & CEP72 & LISD & Live imaging & I & $\checkmark(68)$ \\
\hline 37 & & CEP290/ BBS14 & $\begin{array}{l}\text { No spindle localization (lost } \\
\text { from aMTOCs after NEBD) }\end{array}$ & Live imaging & I & $\checkmark(65)$ \\
\hline 38 & & LRRC36 & LISD & Live imaging & I & $\checkmark(68)$ \\
\hline 39 & & PARD6A/ PAR6A & No spindle localization & Live imaging & I & $\checkmark(82)$ \\
\hline 40 & & PCM1 & LISD & IF, live imaging & I & $\checkmark(52) / x$ \\
\hline 41 & & SSX2IP & No spindle localization & IF & I & $\begin{array}{c}\checkmark(\text { e.g. PMID } \\
23435261)\end{array}$ \\
\hline 42 & \multirow{3}{*}{$\begin{array}{c}\text { Minus-end } \\
\text { binding } \\
\text { proteins }\end{array}$} & CAMSAP3 & LISD & IF & 1 & $\checkmark(48)$ \\
\hline 43 & & KANSL3 & Spindle microtubules, LISD & IF & I & $\begin{array}{c}\checkmark \text { (e.g. PMID } \\
26243146) \\
\end{array}$ \\
\hline 44 & & MCRS1 & aMTOCs & IF & / & $\begin{array}{c}\checkmark(\text { e.g. PMID } \\
26849376)\end{array}$ \\
\hline 45 & \multirow{4}{*}{$\begin{array}{l}\text { Dynein- } \\
\text { related } \\
\text { proteins }\end{array}$} & CLIP170 & Spindle microtubules & Live imaging & I & $x$ \\
\hline 46 & & DCTN1/ P150glued & $\begin{array}{l}\text { Kinetochores, spindle } \\
\text { microtubules }\end{array}$ & IF, live imaging & I & $\begin{array}{c}\checkmark(\text { e.g. PMID } \\
28572454) / \checkmark(81)\end{array}$ \\
\hline 47 & & DCTN2/ dynamitin & $\begin{array}{l}\text { Kinetochores, spindle } \\
\text { microtubules }\end{array}$ & IF, live imaging & 114 & $\begin{array}{c}\checkmark(\text { e.g. PMID } \\
15043813) / \checkmark(82) \\
\end{array}$ \\
\hline 48 & & $\mathrm{DHC}$ & $\begin{array}{c}\text { Kinetochores, spindle } \\
\text { microtubules }\end{array}$ & IF & l & $\begin{array}{c}\checkmark \text { (e.g. PMID } \\
25517096) \\
\end{array}$ \\
\hline
\end{tabular}




\begin{tabular}{|c|c|c|c|c|c|c|}
\hline 49 & & HOOK3 & LISD & IF, live imaging & 1 & $x / \checkmark(74)$ \\
\hline 50 & & LIS1 & Kinetochores & IF & 1 & $x$ \\
\hline 51 & & NDE1 & LISD & IF & I & $\begin{array}{l}\sqrt{ } \text { (e.g. PMID } \\
21394081 \text { ) }\end{array}$ \\
\hline 52 & & NDEL1 & LISD & IF & 1 & $x$ \\
\hline 53 & & NUMA1/ NuMA & aMTOCs & IF & 115 & $\begin{array}{l}\sqrt{ } \text { (e.g. PMID } \\
\text { 26656453) }\end{array}$ \\
\hline 54 & & SPDL1/ spindly & Kinetochores, LISD & IF & 1 & $\checkmark(53)$ \\
\hline 55 & $\begin{array}{l}\text { Microtubule- } \\
\text { related }\end{array}$ & ASPM & Spindle microtubules & IF & 116 & $\begin{array}{l}\sqrt{ } \text { (e.g. PMID } \\
\text { 28436967) }\end{array}$ \\
\hline 56 & proteins & BUGZ & No spindle localization & Live imaging & $/$ & $\sqrt{ }(66)$ \\
\hline 57 & & CLTC/ CHC17 & LISD & IF, live imaging & I & $\begin{array}{c}\checkmark \text { (e.g. PMID } \\
12960147) / \checkmark \\
(21)\end{array}$ \\
\hline 58 & & CKAP5/ chTOG & LISD & IF, live imaging & 1 & $x / \sqrt{ }(72)$ \\
\hline 59 & & GTSE1 & LISD & IF, live imaging & I & $\checkmark(20) / \checkmark(72)$ \\
\hline 60 & & FAM29A/ HAUS6 & Spindle microtubules, LISD & IF & 1 & $\checkmark(49)$ \\
\hline 61 & & KIF2A & $\begin{array}{l}\text { Kinetochores, spindle } \\
\text { microtubules }\end{array}$ & IF & 117 & $\begin{array}{l}\checkmark \text { (e.g. PMID } \\
18411309)\end{array}$ \\
\hline 62 & & KIF2B & aMTOCs & Live imaging & I & $\checkmark(75)$ \\
\hline 63 & & KIF2C/ MCAK & Kinetochores, LISD & IF & 117 & $\checkmark(50)$ \\
\hline 64 & & KIF20A/ MKLP2 & Spindle microtubules & $\mathrm{IF}$ & I & $\checkmark(51)$ \\
\hline 65 & & MYO10 & Cortical cap, LISD & IF & I & $\begin{array}{l}\checkmark \text { (e.g. PMID } \\
\text { 24443004) }\end{array}$ \\
\hline 66 & & TACC3 & LISD & IF, live imaging & I & $\begin{array}{c}\checkmark \text { (e.g. PMID } \\
29089377) / \checkmark(72)\end{array}$ \\
\hline 67 & & TPX2 & Spindle microtubules & IF, live imaging & 118 & $\begin{array}{c}\checkmark \text { (e.g. PMID } \\
24813613) / \checkmark(86)\end{array}$ \\
\hline 68 & $\begin{array}{l}\text { Microtubule- } \\
\text { related }\end{array}$ & GOLGA2/ GM130 & $\begin{array}{l}\text { Foci around aMTOCs and in } \\
\text { the cytosol }\end{array}$ & $\mathrm{IF}$ & 1 & $\begin{array}{l}\checkmark \text { (e.g. PMID } \\
24859005)\end{array}$ \\
\hline
\end{tabular}




\begin{tabular}{|c|c|c|c|c|c|c|}
\hline 69 & $\begin{array}{l}\text { Golgi } \\
\text { proteins }\end{array}$ & TGOLN2/TGN46 & $\begin{array}{l}\text { Foci around aMTOCs and in } \\
\text { the cytosol }\end{array}$ & IF & / & $\begin{array}{c}\checkmark \text { (e.g. PMID } \\
27861594)\end{array}$ \\
\hline 70 & & TRIP11/ GMAP210 & Spindle microtubules & IF & I & $\begin{array}{c}\checkmark \text { (e.g. PMID } \\
27458799\end{array}$ \\
\hline
\end{tabular}

Table S1 List of centrosomal and spindle pole-related proteins mapped in this study. 
102. I. W. Lee, Y. J. Jo, S. M. Jung, H. Y. Wang, N. H. Kim, S. Namgoong, Distinct roles of Cep192 and Cep152 in acentriolar MTOCs and spindle formation during mouse oocyte maturation. FASEB J. 32, 625-638 (2018). doi: 10.1096/fj.201700559RR; pmid 28970258

103. S. Sonn, G. T. Oh, K. Rhee, Nek2 and its substrate, centrobin/Nip2, are required for proper meiotic spindle formation of the mouse oocytes. Zygote. 19, 15-20 (2011). doi: 10.1017/S0967199410000183; pmid: 20569513

104. C. Baumann, X. Wang, L. Yang, M. M. Viveiros, Error-prone meitoic division and subfertility in mice with oocyte-conditional knockdown of pericentrin. J Cell Sci. 130, 1251-1262 (2017). doi: 10.1242/jcs.196188; pmid: 28193732

105. W. Ma, C. Baumann, M. M. Viveiros, NEDD1 is crucial for meiotic spindle stability and accurate chromosome segregation in mammalian oocytes. Dev Biol. 339, 439-450 (2010). doi: 10.1016/j.ydbio.2010.01.009; pmid: 20079731

106. M. J. Carabatsos, C. M. Combelles, S. M. Messinger, D. F. Albertini, Sorting and reorganization of centrosomes during oocyte maturation in the mouse. Microsc Res Tech. 49, 435-444 (2000). doi: 10.1002/(SICI)1097-0029(20000601)49:5<435:AIDJEMT5>3.0.CO;2-H; pmid: 10842370

107. C. Gueth-Hallonet, C. Antony, J. Aghion, A. Santa-Maria, I. Lajoie-Mazenc, M. Wright, B. Maro, gamma-Tubulin is present in acentriolar MTOCs during early mouse development. $J$ Cell Sci. 105, 157-166 (1993). pmid: 8360270

108. M. J. Palacios, H. C. Joshi, C. Simerly, G. Schatten, Gamma-tubulin reorganization during mouse fertilization and early development. J Cell Sci. 104, 383-389 (1993). pmid: 8505367

109. L. J. Yao, Z. S. Zhong, L. S. Zhang, D. Y. Chen, H. Schatten, Q. Y. Sun, Aurora-A is a critical regulator of microtubule assembly and nuclear activity in mouse oocytes, fertilized eggs, and early embryos. Biol Reprod. 70, 1392-1399 (2004). doi: 10.1095/biolreprod.103.025155; pmid: 14695913

110. P. Solc, V. Baran, A. Mayer, T. Bohmova, G. Panenkova-Havlova, A. Saskova, R. M. Schultz, J. Motlik, Aurora kinase A drives MTOC biogenesis but does not trigger resumption of meiosis in mouse oocytes matured in vivo. Biol Reprod. 87, 85 (2012). doi: 10.1095/biolreprod.112.101014; pmid: 22837479

111. G. Pahlavan, Z. Polanski, P. Kalab, R. Golsteyn, E. A. Nigg, B. Maro, Characterization of polo-like kinase 1 during meiotic maturation of the mouse oocyte. Dev Biol. 220, 392-400 (2000). doi: 10.1006/dbio.2000.9656; pmid: 10753525

112. P. Solc, T. S. Kitajima, S. Yoshida, A. Brzakova, M. Kaido, V. Baran, A. Mayer, P. Samalova, J. Motlik, J. Ellenberg, Multiple requirements of PLK1 during mouse oocyte maturation. PLoS One. 10, e0116783 (2015). doi: 10.1371/journal.pone.0116783; pmid: 25658810

113. J. Du, Y. Cao, Q. Wang, N. Zhang, X. Liu, D. Chen, X. Liu, Q. Xu, W. Ma, Unique subcellular distribution of phosphorylated Plk1 (Ser137 and Thr210) in mouse oocytes

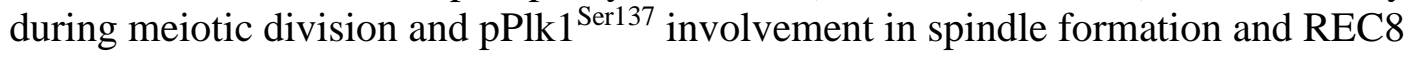


cleavage. Cell Cycle. 14, 3566-3579 (2015). doi: 10.1080/15384101.2015.1100770; pmid: 26654596

114. J. Na, M. Zernicka-Goetz, Asymmetric positioning and organization of the meiotic spindle of mouse oocytes requires CDC42 function. Curr Biol. 16, 1249-1254 (2006). doi: 10.1016/j.cub.2006.05.023; pmid: 16782018

115. J. Lee, T. Miyano, R. M. Moor, Spindle formation and dynamics of gamma-tubulin and nuclear mitotic apparatus protein distribution during meiosis in pig and mouse oocytes. Biol Reprod. 62, 1184-1192 (2000). doi: 10.1095/biolreprod62.5.1184; pmid: 10775165

116. X. L. Xu, W. Ma, Y. B. Zhu, C. Wang, B. Y. Wang, N. An, L. An, Y. Liu, Z. H. Wu, J. H. Tian, The microtubule-associated protein ASPM regulates spindle assembly and meiotic progression in mouse oocytes. PLoS One. 7, e49303 (2012). doi: 10.1371/journal.pone.0049303; pmid: 23152892

117. C. Illingworth, N. Pirmadjid, P. Serhal, K. Howe, G. FitzHarris, MCAK regulates chromosome alignment but is not necessary for preventing aneuploidy in mouse oocyte meiosis I. Development. 137, 2133-2136 (2010). doi: 10.1242/dev.048306; pmid: 20504960

118. S. Brunet, J. Dumont, K. W. Lee, K. Kinoshita, P. Hikai, O. J. Gruss, B. Maro, M. H. Verlhac, Meiotic regulation of TPX2 protein levels governs cell cycle progression in mouse oocytes. PLoS One. 3, e3338 (2008). doi: 10.1371/journal.pone.0003338; pmid: 18833336 
Movie S1 Confocal sections of a mouse metaphase I spindle stained for the LISD (green, TACC3), aMTOCs (magenta, pericentrin) and microtubules (gray, $\alpha$-tubulin).

Movie S2 Time-lapse movie of an acutely nocodazole-treated mouse metaphase I oocyte expressing TACC3-mClover3 (green, to label the LISD) and EB3-3 $\times$ mCherry (magenta, to label microtubules).

Movie S3 Time-lapse movie of fusing spherical condensates in an acutely nocodazole-treated mouse metaphase I oocyte expressing TACC3-mClover3 (gray, to label the spherical condensates).

Movie S4 Time-lapse movie of meiotic maturation of a control mouse oocyte microinjected with mClover3-MAP4-MTBD (green, to label microtubules), CEP192-mScarlet (magenta, to label aMTOCs), H2B-miRFP (blue, to label chromosomes), Trim21 and control IgG.

Movie S5 Time-lapse movie of meiotic maturation of a TACC3-depleted mouse oocyte microinjected with mClover3-MAP4-MTBD (green, to label microtubules), CEP192-mScarlet (magenta, to label aMTOCs), H2B-miRFP (blue, to label chromosomes), Trim21 and anti-TACC3.

Movie S6 Time-lapse movie of meiotic maturation of a TACC3( $\triangle \mathrm{NT})$-TACC3-depleted mouse oocytes microinjected with mClover3-MAP4-MTBD (green, to label microtubules), CEP192- 
mScarlet (magenta, to label aMTOCs), H2B-miRFP (blue, to label chromosomes), Trim21, antiTACC 3 and TACC $3(\triangle N T)$.

Movie S7 Confocal sections of a calcium-treated metaphase I spindle in a control mouse oocyte microinjected with Trim21 and control IgG stained for microtubules (gray, $\alpha$-tubulin) and chromosomes (magenta, Hoechst).

Movie S8 Confocal sections of a calcium-treated metaphase I spindle in a TACC3-depleted mouse oocyte microinjected with Trim 21 and anti-TACC3 stained for microtubules (gray, $\alpha$-tubulin) and chromosomes (magenta, Hoechst).

Movie S9 Confocal sections of a metaphase I spindle in a control mouse oocyte microinjected with PRC1-3×mClover3, Trim21 and control IgG stained for interpolar microtubules (gray and green, GFP) and K-fibers (magenta, TPX2).

Movie S10 Confocal sections of a metaphase I spindle in a TACC3-depleted mouse oocyte microinjected with PRC1-3×mClover3, Trim21 and anti-TACC3 stained for interpolar microtubules (gray and green, GFP) and K-fibers (magenta, TPX2). 


\section{Author contributions}

For publication 1 (Acute and rapid degradation of endogenous proteins by Trim-Away), I wrote the manuscript with Dean Clift and Melina Schuh, simplified and optimized the methods for conducting Trim-Away in mouse oocytes and early embryos, and optimized the peptide preincubation assay and mRNA preparation.

For publication 2 (A liquid-like spindle domain promotes acentrosomal spindle assembly in mammalian oocytes), I wrote the manuscript with Bianka Seres and Melina Schuh, performed all experiments and analyzed the data with the help of Bianka Seres with the following exceptions: Anna Steyer prepared electron samples with me and performed FIB-SEM; Eike Mönnich wrote all in-house-developed scripts and plugins and analyzed comet velocities with Bianka Seres; Dean Clift initiated the systematic analysis of protein localization and characterization of the LISD; Anastasija Pejkovska purified recombinant proteins and optimized in vitro droplet assembly with me. 


\section{Summary of the results}

\section{Identification of the LISD: A liquid-like meiotic spindle domain}

As immunoprecipitation followed by mass spectrometry is not feasible without at least thousands of mouse oocytes, I decided to dissect the molecular composition of the LISD by performing a protein localization screen in live and fixed mouse oocytes. I reasoned that the LISD is in close proximity of the spindle, so I selected 70 centrosomal and spindle-related proteins (publication 2

Fig. 1A). Other than TACC3, 18 other proteins also localized to the LISD: centrosomal proteins (AKAP450, CEP170 and KIZ), centriolar satellite proteins (CEP72, PCM1 and LRRC36), minusend binding proteins (CAMSAP3 and KANSL3), dynein-related proteins (HOOK3, NDE1, NDEL1 and SPDL1), and proteins that control microtubule nucleation and stability (CHC17, chTOG, GTSE1, HAUS6, MCAK and MYO10). Moreover, the LISD was present in the spindle of cow, pig and sheep oocytes, but not in wildtype or centrosome-depleted somatic cells (publication 2 Fig. 1C, and fig. S3, A to D).

\section{The LISD was a liquid-like condensate}

To characterize the LISD, I initially imaged the LISD at high temporal resolution. Interestingly, I observed fusions between the droplet-like protrusions around the spindle poles (publication 2 Fig. 3A). As these protrusions existed in a spindle region that is largely devoid of microtubules, I asked whether the LISD can be maintained independently of microtubules. Upon the acute addition of nocodazole, a microtubule-depolymerizing drug, the LISD was transiently maintained, but then eventually disappeared and reassembled into new spherical condensates (publication 2 Fig. 3B). 
These nocodazole-induced condensates also underwent fusions (publication 2 Fig. 3D). In addition, the LISD displayed other properties reminiscent of liquids: focused ion beam-scanning electron microscopy (FIB-SEM) revealed the absence of a surrounding membrane (publication 2 fig. S13); half fluorescence recovery after photobleaching (FRAP) and photoactivation revealed the rearrangement of its components within the structure (publication 2 Fig. 3, E and F); and FRAP revealed the rapid exchange of its components with the cytosol (publication 2 fig. S14, A and B). In further support of its liquid-like status, the LISD could be reversibly disassembled and reassembled upon nocodazole addition and washout (publication 2 Fig. 3B, and fig. S15A), was perturbed by 1,6-hexanediol that disrupts weak hydrophobic interactions ${ }^{63}$ (publication 2 Fig. 3G, and fig. S15, B and C), and was not stained by thioflavin T that detects amyloid-like interactions ${ }^{64}$ (publication 2 Fig. S15D).

\section{AURA kinase drove LISD assembly}

As the LISD was timely assembled with the spindle during meiosis (publication 2 Fig. 2, A and B), I asked whether the regulatory kinases that control spindle assembly in multiple systems also drives LISD assembly ${ }^{65}$. To this end, I utilized well-characterized, small molecule inhibitors against these regulatory kinases. Whereas pharmacological inhibition of PLK1 and PLK4 did not interfere LISD assembly, inhibition of AURA disrupted LISD assembly (publication $\mathbf{2}$ fig. S4, A to $\mathbf{E})$.

\section{Optimization of Trim-Away in mammalian oocytes}


Of the LISD proteins that I identified, there are two known mitotic substrates of AURA: TACC3 and GTSE1 ${ }^{66-69}$. To examine their role in LISD assembly, it was possible to deplete them early at follicle stage using $\mathrm{RNAi}^{70}$ or at any desired stage using Trim-Away. Trim-Away was chosen instead of RNAi because the proteins may have additional role during follicle development, and depleting them early at follicle stage may complicate the interpretation of phenotypes at later stages. However, one issue with the original Trim-Away protocol was that it requires separate microinjections of TRIM21 mRNA and antibody (publication 1 Fig. 3B), compromising the survival and developmental rates of oocytes. To circumvent this, I established an alternative protocol that allows separate delivery of mRNA and antibody in one single microinjection (publication 1 Fig. 3, A and C).

\section{TACC3 and CHC17 were also essential for LISD assembly}

Both TACC3 and GTSE1 were successfully depleted using Trim-Away (publication 2 figs. S5, A to D, and S7A). Whereas GTSE1 depletion only had a minor effect on LISD assembly (publication 2 fig. S7B), TACC3 depletion fully disassembled the LISD and led to the dispersion of multiple LISD proteins from the spindle (publication 2 fig. S6). As TACC3 binds to microtubules together with $\mathrm{CHC} 17^{71,72}$, I asked whether CHC17 is also critical to LISD assembly. Similar to TACC3 depletion, CHC17 depletion completely disassembled the LISD (publication 2 fig. S8, A and B). And consistent with the codependence of LISD assembly on TACC3 and CHC17, TACC3 mutants ${ }^{72}$ that are unable to interact with $\mathrm{CHC} 17$ (publication 2 fig. S8C) or form the microtubule-binding interface with $\mathrm{CHC} 17$ (publication 2 fig. S8D) were not incorporated into the LISD. 


\section{TACC3 phase-separated via its $\mathrm{N}$ terminus}

Although TACC3 binding to microtubules seemed to be required for LISD assembly (publication 2 fig. S8D), the nocodazole-induced condensates could persist in the absence of microtubules (publication 2 Fig. 3B). Therefore I asked whether both TACC3 and CHC17 are essential for maintaining the nocodazole-induced condensates. Surprisingly, TACC3 depletion, but not CHC17 depletion, disassembled the nocodazole-induced condensates (publication 2 fig. S16, A and B). I thus tested whether TACC3 alone can phase-separate in vitro. Strikingly, recombinant TACC3 could self-organize into spherical droplets in the presence of the molecular crowding agent polyethylene glycol (PEG) publication 2 fig. S17, A to C). These droplets could wet glass surfaces, undergo fusion, exclude $70 \mathrm{kDa}$ dextran and rapidly recover after photobleaching (publication 2 Fig. 4, A to D, and fig. S17D). In silico analysis predicted that both human and mouse TACC3 contain a disordered $\mathrm{N}$ terminus and a structured, coiled-coil-containing $\mathrm{C}$ terminus (publication 2 Fig. 4E). As both disordered and coiled-coil domains have been implicated in driving phase separation in recent studies ${ }^{73}$, I purified different recombinant TACC 3 fragments to test which region(s) is/are sufficient for phase separation in vitro. Whereas the $\mathrm{N}$ terminus fragment of TACC3 self-organized into spherical droplets as the full length TACC3, the $\mathrm{C}$ terminus fragment self-organized into network-like, filamentous structures (publication 2 Fig. 4F). Consistent with the in vitro data, expression of the $\mathrm{C}$ terminus fragment of TACC 3 failed to restore LISD assembly in TACC3-depleted oocytes, but assembled network-like, filamentous structures at the spindle poles and along K-fibres (publication 2 Fig. 4, $\mathbf{G}$ and $\mathbf{H}$ ).

The LISD promoted acentrosomal spindle formation by sequestering microtubule regulatory factors 
Whereas BuGZ, PLK4 and SPD5 are proposed to promote microtubule nucleation in centrosomal spindles by locally enriching tubulin dimers ${ }^{56-58}$, the LISD did not appear to concentrate tubulin dimers (publication 2 fig. S18, A to E). Instead, TACC3 depletion altered microtubule dynamics: microtubule growth rates were significantly reduced, and their overall turnover was significantly increased (publication 2 fig. S19, A to D). I then hypothesized that the LISD locally sequesters microtubule regulatory factors to promote acentrosomal spindle assembly. To investigate this hypothesis, I disrupted the LISD by three different means: AURA inhibition, TACC3 depletion and $\mathrm{CHC} 17$ depletion. All these treatments caused a severe reduction in total microtubule intensity and spindle volume to around half of the values in control oocytes (publication 2 Fig. 5, A to C; figs. S20, A to E, and S21, A to I). Furthermore, AURA inhibition and TACC3 depletion in cow oocytes similarly led to a severe reduction in microtubules (publication 2 Fig. 5, D to F, and fig. S24).

To uncover LISD-specific functions in oocytes, I utilized TACC3( $\triangle \mathrm{NT})$, the $\mathrm{C}$ terminus fragment that lacks the $\mathrm{N}$ terminus required for phase separation and LISD assembly (publication 2 Fig. 4, F to $\mathbf{H})$ but has been shown to rescue phenotypes of TACC3 depletion in Xenopus mitotic extracts and in mitotic cells ${ }^{69,72}$. Remarkably, TACC3 $(\Delta \mathrm{NT})$ did not rescue the reduction in total microtubule intensity in TACC3-depleted oocytes (publication 2 Fig. 6, A to C). Although not as strongly as in TACC3-depleted oocytes, the spindle volume in TACC3( $\triangle \mathrm{NT})$-TACC3-depleted oocytes was still significantly reduced when compared with that in wildtype oocytes (publication 2 Fig. 6, A to C). By contrast, phenotypes related to K-fibers, such as reduced progression into anaphase and presence of lagging chromosomes in TACC3-depleted oocytes, were largely rescued by $\mathrm{TACC}(\Delta \mathrm{NT})$ (publication 2 fig. S25, D to $\mathbf{G})$. 


\section{K-fibers and interpolar microtubules were depleted}

To investigate further the substantial loss of spindle microtubules, I examined K-fibers and interpolar microtubules, which are the two major populations of spindle microtubules ${ }^{74}$.

To assess K-fibers, oocytes were briefly placed on ice to depolymerize dynamic, non-kinetochorebound microtubules, leaving behind the more stable, kinetochore-bound microtubules that are bundled to form K-fibers ${ }^{75}$. TACC3-depleted oocytes had significantly less K-fibers (publication 2 Fig. 7, A to C).

As there are no standard assays for evaluating interpolar microtubules, I assessed interpolar microtubules by three different approaches. First, I treated oocytes with calcium ions, which destabilize microtubules ${ }^{76}$ but preserve K-fibers and stable interpolar microtubules in mouse oocytes ${ }^{77}$. A more severe microtubule loss was observed when compared with the cold-stable assay (publication 2 Fig. 7, E and F). Second, I established a fluorescent reporter for the microtubule cross-linker PRC1, which associates with interpolar microtubules in mitotic cells ${ }^{78}$. In line with the calcium-stable assay, PRC1-labeled microtubules were severely reduced in TACC3-depleted oocytes (publication 2 Fig. 7, G and $\mathbf{H}$ ). Third, I examined the central spindle. During anaphase, K-fibers were depolymerized, leaving behind largely the interpolar microtubules in the central spindle $^{79}$. The total intensity and volume of the central spindle were significantly reduced in TACC3-depleted oocytes (publication 2 fig. S26, A to C). 


\section{Discussion}

\section{An optimized protocol for Trim-Away in mammalian oocytes}

In the original Trim-Away protocol, GV-arrested oocytes are first microinjected with TRIM21 mRNA and then allowed to rest for three to four hours for protein expression and recovery from microinjection $^{27}$. Before released from GV arrest, oocytes are microinjected with antibody to induce target degradation ${ }^{27}$. The two rounds of microinjection markedly reduce the survival and developmental rates of oocytes and are often challenging even for experienced microinjectionist. In the new protocol, oocytes are co-microinjected with TRIM21 mRNA and antibody. This is achieved with the use of a less viscous silicone oil, which prevents prior mixing of the TRIM21 mRNA solution and antibody solution in the loading capillary and in the microinjection needle. This eliminates the need of the second microinjection, making Trim-Away experiments as simple as other routine experiments that require only a single microinjection. Besides, target degradation begins as TRIM21 mRNA is translated, allowing more time for the oocytes to thoroughly degrade the target before released from GV arrest.

\section{A novel principle of spindle assembly}

My work uncover an unprecedented principle of acentrosomal spindle assembly in mammalian oocytes: Microtubule regulatory factors are organized into a liquid-like domain to promote meiotic spindle assembly in the absence of centrosomes. Enriching these factors in local proximity of the spindle may be particularly crucial for large cells such as oocytes, where these factors would otherwise be dispersed throughout the large ooplasm. Liquid-liquid phase separation represents an 
idea principle for such a purpose: It sequesters factors within proximity of spindle microtubules, but still allows them to diffuse dynamically along the spindle. This could help to promote the even distribution of these factors throughout the spindle, and to titrate their local concentration in order to promote efficient spindle assembly within the large cytoplasmic volume.

\section{Does the LISD play a role beyond spindle assembly in mammalian oocytes?}

Although many of the LISD proteins have been studied extensively in mitotic cells, structures that resemble the LISD have not been reported in other cell types so far and were not observed in mouse embryonic fibroblasts on my hands. As suggested by one of the reviewers for publication 2, I even attempted to deplete the centrosomes in somatic cells using centrinone, an inhibitor of PLK4 kinase and centriole duplication, and examine TACC 3 in these centrosome-depleted cells. Nevertheless, I did not observe any liquid-like structures in these acentrosomal spindles. Hence, the LISD is likely an oocyte-specific feature. Notably though, when TACC3 is highly overexpressed in somatic cells, it forms large spherical structures that can associate with the mitotic spindle and persist in the presence of nocodazole ${ }^{80}$. However, in line with my observations, such structures were absent when TACC3 is expressed at endogenous level ${ }^{80,81}$. Whether these structures are physiologically relevant and whether they form by phase separation remain unclear. Therefore, mammalian oocytes appear to utilize the same set of microtubule regulatory factors as mitotic cells, but organize them into a prominent liquid-like domain.

\section{The first study of 'fatty' mammalian oocytes using high-resolution light microscopy}


Cow, pig and sheep oocytes have a lot of lipid droplets of different sizes in their cytoplasm, which heavily scatters light and only allows visualization of less than twenty microns below the cortex. To image these 'fatty' mammalian oocytes in the past, they were pre-permeabilized with a detergent-containing solution that stabilizes microtubules before fixation ${ }^{82}$. However, variations between staining of individual oocytes were huge and whether the spindles remain native is unclear. Inspired by a recent optimization for CLARITY ${ }^{83}$, a tissue clearing technique, I established an optical clearing protocol based on the use of lipase from Candida rugose that has less protease contamination when compared with lipase from porcine pancreas. With one step of lipase treatment added to the routine immunostaining protocol, this allowed visualization of the full spindle and even the whole cytoplasmic actin network (unpublished) in these 'fatty' mammalian oocytes. I believe this technique will be a game changer for studies that involve 'fatty' mammalian oocytes.

\section{The first volume electron microscopy of mammalian oocytes}

To demonstrate the absence of a membrane surrounding the LISD, I decided to use FIB-SEM rather than performing immunostaining for markers of different membranes, which could be compromised due to the extraction with detergent. However, FIB-SEM, or electron microscopy in general, of the LISD or even the spindle was not trivial because they are largely composed of proteins and thus contrasted poorly by most heavy metal stains, which are lipophilic. In the past transmission electron microscopy studies of mammalian oocytes, sufficient contrast was only obtained after extensive extraction of the cytoplasm ${ }^{37,84}$. Fortunately, after a series of optimizations for the fixation, staining and imaging protocols, sufficient contrast was obtained with reduced osmium-thiocarbohydrazide-osmium staining in oocytes fixed with a mix of formaldehyde and 
glutaraldehyde, which allowed semi-automatic, threshold-based segmentation. In addition, I combined the use of ultrasmall gold-conjugated $\mathrm{Fab}^{85}$ and microinjection to implement immunogold labelling for TACC3 in intact oocytes. Hopefully, these development will lead to more ultrastructural studies of mammalian oocytes in the field.

\section{Which is more centrosome-like: aMTOCs or the LISD?}

During mitotic PCM assembly, long coiled coil proteins such as centrosomin (Drosophila) and SPD-5 (C. elegans) multimerize to form the PCM "scaffold", which in turn recruits PCM "clients" such as tubulin, kinases, etc ${ }^{86-87}$. Similar hierarchy has recently been demonstrated by elegant in vitro reconstitutions using SPD-5, PLK-1, SPD-2, TPXL-1 and ZYG-956. Although centrosomal proteins could be found in both aMTOCs and the LISD, aMTOCs appear to be more centrosomelike because (i) microtubule nucleator $\gamma$-tubulin was present in aMTOCs but not the LISD and (ii) I observed similar 'scaffold'-'client' relationships in aMTOCs but not the LISD using FRAP. Intriguingly, aMTOCs undergo fragmentation and clustering to remodel into different sizes during meiosis, and persist in the presence of nocodazole ${ }^{42}$. Whether aMTOCs are also a type of condensates like the mitotic PCM in Drosophila and C. elegans remains to be investigated ${ }^{56,88}$.

\section{Conditions for phase separation of TACC3 in vitro}

To test if TACC3 could phase-separate in vitro, I added the molecular crowding agent PEG to recombinant TACC3 as described for SPD- $5^{56}$. A concern raised by one of the reviewers was that whether such in vitro conditions are physiologically relevant. This concern is totally valid as the presence or absence of molecular crowding agent could have a big impact on the assemblies 
obtained. Taking SPD-5 as an example of how versatile proteins are, SPD-5 was reported to assemble network-like structures in the absence of PEG in $2015^{87}$. But in 2017, when the authors took into account the crowded environment in the cytoplasm and incubated SPD-5 in the presence of PEG, they obtained droplet-like structures ${ }^{56}$. Back to the case of TACC3, although I used a relatively high concentration of PEG (12\%), I believe our in vitro conditions are justifiable based on two observations. First, I observed droplet-like TACC3 structures in vivo and full-length TACC3 self-organized into droplets in vitro. Second, the C terminus of TACC3 self-organized into network-like structures in vitro, and I observed similar structures in TACC3( $\triangle \mathrm{NT})$-TACC3depleted oocytes. In line with their expected material properties, the network-like structures for $\operatorname{TACC} 3(\triangle \mathrm{NT})$ were resistant to cold treatment, but not the droplet-like structures for full-length TACC3. Such consistency between our in vitro and in vivo data suggests that our in vitro conditions are likely physiologically relevant.

\section{The LISD versus nocodazole-induced condensates}

Although the LISD and nocodazole-induced condensates were similar in many perspectives, such as their morphology, liquid-like properties, AURA dependence and TACC3 dependence, they are different in their sensitivity to 3.5\% 1,6-hexanediol and $\mathrm{CHC} 17$ dependence. Whereas the nocodazole-induced condensates were fully disassembled by the addition of 3.5\% 1,6-hexanediol, the LISD was not. Whereas the LISD was disassembled upon CHC17 depletion, the nocodazoleinduced condensates were not. Together with the fact that the N terminus of TACC 3 was sufficient for phase separation in vitro but not incorporation into the LISD in vivo, one likely explanation is that the $\mathrm{C}$ terminus of $\mathrm{TACC} 3$, which is required for interactions with $\mathrm{CHC} 17$ and the formation of microtubule-binding interface ${ }^{72}$, also plays a role in LISD assembly. Binding of TACC3- 
CHC17 complex to microtubules may help to stabilize the condensates in vivo, thus conferring the LISD a higher resistance to 1,6-hexanediol than the nocodazole-induced condensates.

\section{Phase separation in mitotic spindle assembly versus meiotic spindle assembly}

During mitotic spindle assembly, phase separation has been proposed as a mechanism for enriching tubulin dimers around the centrosomes or existing microtubules to facilitate microtubule nucleation $^{56-58,61}$. However, the LISD did not concentrate tubulin dimers but enrich different microtubule regulatory factors during meiotic spindle assembly. Why mammalian oocytes do not concentrate tubulin dimers as in somatic cells? Recent computational modeling suggests that tubulin dimers are not limiting in large cytoplasmic volume ${ }^{89,90}$, so enriching tubulin dimers may be less an important issue in large oocytes. Intriguingly, a recent study suggest that when tubulin dimers are not limiting, microtubule dynamics becomes a key factor in determining spindle size ${ }^{91}$. If this also applies to mammalian oocytes, this may indeed explain why phase separation of microtubule regulatory factors is used to modulate microtubule dynamics.

\section{PRC1: a novel reporter for interpolar microtubules}

To visualize interpolar microtubules in intact oocytes, I established a fluorescent reporter for the microtubule crosslinker PRC1. In mouse oocytes, whereas TPX2 labeled K-fibers and some interpolar microtubules, PRC1 predominantly labeled interpolar microtubules. Notably, at the spindle midzone, PRC1 was enriched and labeled bundles of overlapping interpolar microtubules that lie below each pair of K-fibers. In mitotic cells, these microtubules were termed the bridging fibers ${ }^{92}$, and it was found that sliding of microtubules within drives spindle pole separation and 
pushes K-fibers poleward ${ }^{93}$. Indeed, a previous study on mouse oocytes also revealed a similar localization for the microtubule-stabilizing protein $\mathrm{HURP}^{46}$. The functions of these bridging fiberlike microtubules in mouse oocytes remain to be determined.

\section{Outlook}

Although this thesis addresses most aspects of the LISD, there are several open questions. First, why TACC 3 phase-separate in oocytes during meiosis, but not in GV oocytes or mitotic cells. This could be due to differences in TACC 3 concentration in the cytoplasm, or differences in AURA activity. Second, how AURA regulates LISD assembly. Preliminary experiments with S558 phosphomutants of TACC3 suggested that AURA does not regulate LISD assembly through the canonical S558 phosphorylation (unpublished). Once the critical phosphorylation site(s) is/are identified, we could construct phosphomutants that fail to phase-separate and repeat the rescue experiments as for TACC $3(\triangle \mathrm{NT})$ to further delineate LISD-specific functions. Third, how the LISD could be integrated into the current picture of meiotic spindle assembly. After the initial microtubule nucleation from aMTOCs and the RanGTP pathway ${ }^{41,44,45}$, the LISD may help stabilizing microtubules nucleated from either or both pathways. Fourth, whether the LISD is present on the meiotic spindle in human oocytes. Unlike other mammalian oocytes, the spindle in human oocytes is particularly unstable ${ }^{49}$. It will be interesting if the LISD is absent in human oocytes. And finally, whether disturbance in the LISD occurs naturally, for instance, with aging. Disturbance in the LISD likely has a negative impact on the meiotic spindle, which could be a factor contributing to infertility in females. 


\section{References}

1. B. E. Housden, M. Muhar, M. Gemberling, C. A. Gersbach, D. Y. Stainier, G. Seydoux, S. E. Mohr, J. Zuber, N. Perrimon, Loss-of-function genetic tools for animal models: cross-species and cross-platform differences. Nat. Rev. Genet. 18, 24-40 (2017).

2. T. Gaj, C. A. Gersbach, C. F. Barbas, ZFN, TALEN, and CRISPR/Cas-based methods for genome engineering. Trends Biotechnol. 31, 397-405 (2013).

3. M. Boettcher, M. T. McManus, Choosing the right tool for the job: RNAi, TALEN, or CRISPR. Mol. Cell 58, 575-585 (2015).

4. R. C. Wilson, J. A. Doudna, Molecular mechanisms of RNA interference. Annu. Rev. Biophys. 42, 217-239 (2013).

5. J. S. Eisen, J. C. Smith, Controlling morpholino experiments: don’t stop making antisense. Development 135, 1735-1743 (2008).

6. J. N. Savas, B. H. Toyama, T. Xu, J. R. Yates, M. W. Hetzer, Extremely long-lived nuclear pore proteins in the rat brain. Science 335, 942 (2012).

7. B. H. Toyama, J. N. Savas, S. K. Park, M. S. Harris, N. T. Ingolia, J. R. Yates, M. W. Hetzer, Identification of long-lived proteins reveals exceptional stability of essential cellular structures. Cell 154, 971-982 (2013).

8. B. H. Toyama, M. W. Hetzer, Protein homeostasis: live long, won't prosper. Nat. Rev. Mol. Cell. Biol. 14, 55-61 (2013). 
9. W. A. Weiss, S. S. Taylor, K. M. Shokat, Recognizing and exploiting differences between RNAi and small-molecule inhibitors. Nat. Chem. Biol. 3, 739-744 (2007).

10. M. A. El-Brolosy, D. Y. R. Stainier, Genetic compensation: a phenomenon in search of mechanisms. PLoS Genet. 13, e1006780 (2017).

11. K. M. Sakamoto, K. B. Kim, A. Kumagai, F. Mercurio, C. M. Crews, R. J. Deshaies, Protacs: chimeric molecules that target proteins to the Skp1-Cullin-F box complex for ubiquitination and degradation. Proc. Natl. Acad. Sci. U S A 98, 8554-8559 (2001).

12. X. Fan, W. Y. Jin, J. Lu, J. Wang, Y. T. Wang, Rapid and reversible knockdown of endogenous proteins by peptide-directed lysosomal degradation. Nat. Neurosci. 17, 471-480 (2014).

13. K. Nishimura, T. Fukagaw, H. Takisawa, T. Kakimoto, M. Kanemaki, An auxin-based degron system for the rapid depletion of proteins in nonplant cells. Nat. Methods 6, 917-922 (2009).

14. E. Caussinus, O. Kanca, M. Affolter, Fluorescent fusion protein knockout mediated by antiGFP nanobody. Nat. Struct. Mol. Biol. 19, 117-121 (2011).

15. S. T. Armenti, L. L. Lohmer, D. R. Sherwood, J. Nance, Repurposing an endogenous degradation system for rapid and targeted depletion of C. elegans proteins. Development 141, 4640-4647 (2014).

16. L. A. Banaszynski, L. C. Chen, L. A. Maynard-Smith, A. G. Ooi, T. J. Wandless, A rapid, reversible, and tunable method to regulate protein function in living cells using synthetic small molecules. Cell 126, 995-1004 (2006). 
17. T. K. Neklesa, H. S. Tae, A. R. Schneekloth, M. J. Stulberg, T. W. Corson, T. B. Sundberg, K. Raina, S. A. Holley, C. M. Crews, Small-molecule hydrophobic tagging-induced degradation of HaloTag fusion proteins. Nat. Chem. Biol. 7, 538-543 (2011).

18. M. S. Robinson, D. A. Sahlender, S. D. Foster, Rapid inactivation of proteins by rapamycininduced rerouting to mitochondira. Dev. Cell 18, 324-331 (2010).

19. I. Dikic, Proteasomal and autophagic degradation systems. Annu. Rev. Biochem. 86, 193-224 (2017).

20. R. Sahu, S. Kaushik, C. C. Clement, E. S. Cannizzo, B. Scharf, A. Follenzi, I. Potolicchio, E. Nieves, A. M. Cuervo, L. Santambrogio, Microautophagy of cytosolic proteins by late endosomes. Dev. Cell 20, 131-139 (2011).

21. L. C. James, Intracellular antibody immunity and the cytosolic Fc receptor TRIM21. Curr. Top. Microbiol. Immunol. 382, 51-66 (2014).

22. W. A. McEwan, L. C. James, TRIM21-dependent intracellular antibody neutralization of virus infection. Prog. Mol. Biol. Transl. Sci. 129, 167-187 (2015).

23. S. Foss, R. Watkinson, I. Sandlie, L. C. James, J. T. Andersen, TRIM21: a cytosolic Fc receptor with broad antibody isotype specificity. Immunol. Rev. 268, 328-339 (2015).

24. A. J. Fletcher, L. C. James, Coordinated neutralization and immune activation by the cytosolic antibody receptor TRIM21. J. Virol. 90, 4856-4859 (2016).

25. W. A. McEwan, Surveillance for intracellular antibody by cytosolic Fc receptor TRIM21. Antibodies 5, 21 (2016). 
26. D. A. Rhodes, D. A. Isenberg, TRIM21 and the function of antibodies inside cells. Trends Immunol. 38, 916-926 (2017).

27. D. Clift, W. A. McEwan, L. I. Labzin, V. Konieczny, B. Mogessie, L. C. James, M. Schuh, A method for the acute and rapid degradation of endogenous proteins. Cell 171, 1692-1706 (2017).

28. X. Chen, M. Liu, H. Lou, Y. Lu, M. T. Zhou, R. Ou, Y. Xu, K. F. Tang, Degradation of endogenous proteins and generation of a null-like phenotype in zebrafish using Trim-Away technology. Genome Biol. 20, 19 (2019).

29. J. Rojas, M. Chavez-Castillo, L. C. Olivar, M. Calvo, J. Mejias, M. Rojas, J. Morillo, V. Bermudez, Physiologic course of female reproductive function: a molecular look into the prologue of life. J. Pregnancy 2015, 715735 (2015).

30. N. Rimon-Dahari, L. Yerushalmi-Heinemann, L. Alyagor, N. Dekel, “Ovarian folliculogenesis" in Molecular Mechanisms of Cell Differentiation in Gonad Development (Springer International Publishing, Switzerland, 2016), pp.167-190.

31. Y. Choi, A. Rajkovic, Genetics of early mammalian folliculogenesis. Cell Mol. Life Sci. 63, 579-590 (2006).

32. K. J. Hutt, D. F. Albertini, An oocentric view of folliculogenesis and embryogenesis. Reprod. Biomed. Online 14, 758-764 (2007).

33. E. E. Telfer, M. McLaughlin, Natural history of the mammalian oocyte. Reprod. Biomed. Online 15, 288-95 (2007).

34. J. R. Von Stetina, T. L. Orr-Weaver, Developmental control of oocyte maturation and egg activation in metazoan models. Cold Spring Harb. Perspect. Biol. 3, a005553 (2011). 
35. J. E. Holt, S. I. Lane, K. T. Jones, The control of meiotic maturation in mammalian oocytes. Curr. Top. Dev. Biol. 102, 207-226 (2013).

36. L. Bury, P. A. Coelho, D. M. Glover, From meiosis to mitosis: the astonishing flexibility of cell division mechanisms in early mammalian development. Curr. Top. Dev. Biol. 120, 125-171 (2016).

37. D. Szollosi, P. Calarco, R. P. Donahue, Absence of centrioles in the first and second meiotic spindles of mouse oocytes. J. Cell Sci. 11, 521-541 (1972).

38. G. Manandhar, H. Schatten, P. Sutovsky, Centrosome reduction during gametogenesis and its significance. Biol. Reprod. 72, 2-13 (2005).

39. P. T. Conduit, A. Wainman, J. W. Raff, Centrosome function and assembly in animal cells. Nat. Rev. Mol. Cell Biol. 16, 611-624 (2015).

40. M. Luksza, I. Queguigner, M. H. Verlhac, S. Brunet, Rebuilding MTOCs upon centriole loss during mouse oogenesis. Dev. Biol. 382, 48-56 (2013).

41. M. Schuh, J. Ellenberg, Self-organization of MTOCs replaces centrosome function during acentrosomal spindle assembly in live mouse oocytes. Cell 130, 484-498 (2007).

42. D. Clift, M. Schuh, A three-step MTOC fragmentation mechanism facilitates bipolar spindle assembly in mouse oocytes. Nat. Commun. 6, 7217 (2015).

43. T. Cavazza, I. Vernos, The RanGTP pathway: from nucleo-cytoplasmic transport to spindle assembly and beyond. Front. Cell Dev. Biol. 3, 82 (2015). 
44. J. Dumont, S. Petri, F. Pellegrin, M. E. Terret, M. T. Bohnsack, P. Rassinier, V. Georget, P. Kalab, O. J. Gruss, M. H. Verlhac, A centriole- and RanGTP-independent spindle assembly pathway in meiosis I of vertebrate oocytes. J. Cell Biol. 176, 295-305 (2007).

45. C. Baumann, X. Wang, L. Yang, M. M. Viveiros, Error-prone meiotic division and subfertility in mice with oocyte-conditional knockdown of pericentrin. J. Cell Sci. 130, 12511262 (2017).

46. M. Breuer, A. Kolano, M. Kwon, C. C. Li, T. F. Tsai, D. Pellman, S. Brunet, M. H. Verlhac, HURP permits MTOC sorting for robust meiotic spindle bipolarity, similar to extra centrosome clustering in cancer cells. J. Cell Biol. 191, 1251-1260 (2010).

47. T. S. Kitajima, M. Ohsugi, J. Ellenberg, Complete kinetochore tracking reveals error-prone homologous chromosome biorientation in mammalian oocytes. Cell 146, 568-581 (2011).

48. A. Z. Balboula, A. L. Nguyen, A. S. Gentilello, S. M. Quartuccio, D. Drutovic, P. Solc, K. Schindler, Haspin kinase regulates microtubule-organizing center clustering and stability through Aurora kinase C in mouse oocytes. J. Cell. Sci. 129, 3648-3660 (2016).

49. Z. Holubcova, M. Blayney, K. Elder, M. Schuh, Error-prone chromosome-mediated spindle assembly favors chromosome segregation defects in human oocytes. Science 348, 1143-1147 (2015).

50. C. P. Brangwynne, C. R. Eckmann, D. S. Courson, A. Rybarska, C. Hoege, J.Gharakhani, F. Julicher, A. A. Hyman, Germline P granules are liquid droplets that localize by controlled dissolution/condensation. Science 324, 1729-1732 (2009). 
51. C. P. Brangwynne, T. J. Mitchison, A. A. Hyman, Actie liquid-like behavior of nucleoli determines their size and shape in Xenopus laevis oocytes. Proc. Natl. Acad. Sci. U S A 108, 4334-4339 (2011).

52. A. Molliex, J. Temirov, J. Lee, M. Coughlin, A. P. Kanagaraj, H. J. Kim, T. Mittag, J. P. Taylor, Phase separation by low complexity domains promotes stress granule assembly and drives pathological fibrillization. Cell 163, 123-133 (2015).

53. A. A. Hyman, C. A. Weber, F. Julicher, Liquid-liquid phase separation in biology. Annu. Rev. Cell Dev. Biol. 30, 39-58 (2014).

54. S. F. Banani, H. O. Lee, A. A. Hyman, M. K. Rosen, Biomolecular condensates: organizers of cellular biochemistry. Nat. Rev. Mol. Cell Biol. 18, 285-298 (2017).

55. J. B. Woodruff, Assembly of mitotic structures through phase separation. J. Mol. Biol. 430, 4762-4772 (2018).

56. J. B. Woodruff, B. Ferreira Gomes, P. O. Widlund, J. Mahamid, A. Honigmann, A. A. Hyman, The centrosome is a selective condensate that nucleates microtubules by concentrating tubulin. Cell 169, 1066-1077 (2017).

57. S. Montenegro Gouveia, S. Zitouni, D. Kong, P. Duarte, B. Ferreira Gomes, A. L. Sousa, E. M. Tranfield, A. Hyman, J. Loncarek, M. Bettencourt-Dias, PLK4 is a microtubule-associated protein that self-assembles promoting de novo MTOC formation. J. Cell Sci. 132, jcs219501 (2018).

58. H. Jiang, S. Wang, Y. Huang, X. He, H. Cui, X. Zhu, Y. Zheng, Phase transition of spindleassociated protein regulate spindle apparatus assembly. Cell 163, 108-122 (2015). 
59. Y. Huang, T. Li, S. C. Ems-McClung, C. E. Walczak, C. Prigent, X. Zhu, X. Zhang, Y. Zheng, Aurora A activation in mitosis promoted by BuGZ. J. Cell Biol. 217, 107-116 (2018).

60. A. Hernandez-Vega, M. Braun, L. Scharrel, M. Jahnel, S. Wegmann, B. T. Hyman, S. Alberti, S. Diez, A. A. Hyman, Local nucleation of microtubule bundles through tubulin concentration into a condensed Tau phase. Cell Rep. 20, 2304-2312 (2017).

61. M. R. King, S. Petry, https://www.biorxiv.org/content/10.1101/668426v1 (2019).

62. D. G. Booth, F. E. Hood, I. A. Prior, S. J. Royle, A TACC3/ch-TOG/clathrin complex stabilizes kinetochore fibres by intermicrotubule briding. EMBO J. 30, 906-919 (2011).

63. S. Kroschwald, S. Maharana, A. Simon, Hexanediol: A chemical probe to investigate the material properties of membrane-less compartments. Matters (2017).

64. H. B. Schmidt, D. Gorlich, Transport selectivity of nuclear pores, phase separation, and membraneless organelles. Trends Biochem. Sci. 41, 46-61 (2016).

65. L. Bury, P. A. Coelho, A. Simeone, S. Ferries, C. E. Eyers, P. A. Eyers, M. Zernicka-Goetz, D. M. Glover, Plk4 and Aurora A cooperate in the initiation of acentriolar spindle assembly in mammalian oocytes. J. Cell Biol. 216, 3571-3590 (2017).

66. A. N. Kettenbach, D. K. Schweppe, B. K. Faherty, D. Pechenick, A. A. Pletnev, S. A. Gerber, Quantitative phosphoproteomics identifies substrates and functional modules of Aurora and Polo-like kinase activities in mitotic cells. Sci. Signal. 4, rs5 (2011).

67. T. P. Barros, K. Kinoshita, A. A. Hyman, J. W. Raff, Aurora A activates D-TACC-Msps complexes exclusively at centrosomes to stabilize centrosomal microtubules. J. Cell Biol. 170, 1039-1046 (2005). 
68. K. Kinoshita, T. L. Noetzel, L. Pelletier, K. Mechtler, D. N. Drechsel, A. Schwager, M. Lee, J. W. Raff, A. A. Hyman, Aurora A phosphorylation of TACC3/maskin is required for centrosome-dependent microtubule assembly in mitosis. J. Cell Biol. 170, 1047-1055 (2005).

69. I. Peset, J. Seiler, T. Sardon, L. A. Bejarano, S. Rybina, I. Vernos, Function and regulation of Maskin, a TACC family protein, in microtubule growth during mitosis. J. Cell Biol. 170, 10571066 (2005).

70. S. Pfender, V. Kuznetsov, M. Pasternak, T. Tischer, B. Santhanam, M. Schuh, Live imaging RNAi screen reveals genes essential for meiosis in mammalian oocytes. Nature. 524, 239-242 (2015).

71. C. H. Lin, C. K. Hu, H. M. Shih, Clathrin heavy chain mediates TACC3 targeting to mitotic spindles to ensure spindle stability. J. Cell Biol. 189, 1097-1105 (2010).

72. F. E. Hood, S. J. Williams, S. G. Burgess, M. W. Richards, D. Roth, A. Straube, M. Pfuhl, R. Bayliss, S. J. Royle, Coordination of adjacent domains mediates TACC3-ch-TOG-clathrin assembly and mitotic spindle binding. J. Cell Biol. 202, 463-478 (2013).

73. S. Boeynaems, S. Alberti, N. L. Fawzi, T. Mittag, M. Polymenidou, F. Rousseau, J. Schymkowitz, J. Shorter, B. Wolozin, L. Van Den Bosch, P. Tompa, M. Fuxreiter, Protein phase separation: a new phase in cell biology. Trends Cell Biol. 28, 420-435 (2018).

74. S. Dumont, T. J. Mitchison, Force and length in the mitotic spindle. Curr. Biol. 19, R749R761 (2009).

75. B. R. Brinkley, J. Cartwright, Cold-labile and cold-stable microtubules in the mitotic spindle of mammalian cells. Ann. N Y Acad. Sci. 253, 428-439 (1975). 
76. R. C. Weisenberg, W. J. Deery, The mechanism of calcium-induced microtubule disassembly. Biochem. Biophys. Res. Commun. 102, 924-931 (1981).

77. T. S. Kitajima, M. Ohsugi, J. Ellenberg, Complete kinetochore tracking reveals error-prone homologous chromosome biorientation in mammalian oocytes. Cell 146, 568-581 (2011).

78. J. Kajtez, A. Solomatina, M. Novak, B. Polak, K. Vukusic, J. Rudiger, G. Cojoc, A. Milas, I. Sumanovac Sestak, P. Risteski, F. Tavano, A. H. Klemm, E. Roscioli, J. Welburn, D. Cimini, M. Gluncic, N. Pavin, I. M. Tolic, Overlap microtubules link sister k-fibres and balance the forces on bi-oriented kinetochores. Nat Commun. 7, 10298 (2016).

79. H. Maiato, M. Lince-Faria, The perpetual movements of anaphase. Cell Mol. Life Sci. 67, $2251-2269$ (2010).

80. F. Gergely, C. Karlssson, I. Still, J. Cowell, J. Kilmartin, J. W. Raff, The TACC domain identifies a family of centrosomal proteins that can interact with microtubules. Proc. Natl. Acad. Sci. U S A 97, 14352-14357 (2000).

81. F. M. Nixon, C. Gutierrez-Caballero, F. E. Hood, D. G. Booth, I. A. Prior, S. J. Royle, The mesh is a network of microtubule connectors that stabilizes individual kinetochore fibers of the mitotic spindle. Elife 4 (2015).

82. C. Simerly, G. Schatten, Techniques for localization of specific molecules in oocytes and embryos. Methods Enzymol. 225, 516-553 (1993).

83. M. Lai, X. Li, J. Li, Y. Hu, D. M. Czajkowsky, Z. Shao, Improved clearing of lipid dropletrich tissues for three-dimensional structural elucidation. Acta. Biochim. Biophys. Sin. (Shanghai) 49, 465-467 (2017). 
84. S. Brunet, A. S. Maria, P. Guillaud, D. Dujardin, J. Z. Kubiak, B. Maro, Kinetochore fibers are not involved in the formation of the first meiotic spindle in mouse oocytes, but control the exit from the first meiotic M phase. J. Cell Biol. 146, 1-12 (1999).

85. I. Orlov, A. Schertel, G. Zuber, B. Klaholz, R. Drillien, E. Weiss, P. Schultz, D. Spehner, Live cell immunogold labelling of RNA polymerase II. Sci. Rep. 5, 8324 (2015).

86. P. T. Conduit, Z. Feng, J. H. Richens, J. Baumbach, A. Wainman, S. D. Bakshi, J.

Dobbelaere, S. Johnson, S. M. Lea, J. W. Raff, The centrosome-specific phosphorylation of Cnn by Polo/Plk1 drives Cnn scaffold assembly and centrosome maturation. Dev. Cell 28, 659-669 (2014).

87. J. B. Woodruff, O. Wueseke, V. Viscardi, J. Mahamid, S. D. Ochoa, J. Bunkenborg, P. O. Widlund, A. Pozniakovsky, E. Zanin, S. Bahmanyar, A. Zinke, S. H. Hong, M. Decker, W. Baumeister, J. S. Anderson, K. Oegema, A. A. Hyman, Regulated assembly of a supramolecular centrosome scaffold in vitro. Science 348, 808-812 (2015).

88. Z. Feng, A. Caballe, A. Wainman, S. Johnson, A. F. M. Haensele, M. A. Cottee, P. T. Conduit, S. M. Lea, J. W. Raff, Structural basis for mitotic centrosome assembly in flies. Cell 169, 1078-1089 (2017).

89. M. C. Good, M. D. Vahey, A. Skandarajah, D. A. Fletcher, R. Heald, Cytoplasmic volume modulates spindle size during embryogenesis. Science 342, 856-860 (2013).

90. T. Cavazza, P. Malgaretti, I. Vernos, The sequential activation of the mitotic microtubule assembly pathways favors bipolar spindle formation. Mol. Biol. Cell 27, 2935-2945 (2016). 
91. B. Lacroix, G. Letort, L. Pitayu, J. Salle, M. Stefanutti, G. Maton, A. M. Ladouceur, J. C. Canman, P. S. Maddox, A. S. Maddox, N. Minc, F. Nedelec, J. Dumont, Microtubule dynamics scale with cell size to set spindle length and assembly timing. Dev. Cell 45, 496-511 (2018).

92. B. Polak, P. Risteski, S. Lesjak, I. M. Tolic, PRC1-labeled microtubule bundles and kinetochore pairs show one-to-one association in metaphase. EMBO Rep. 18, 217-230 (2017).

93. K. Vukusic, R. Buda, A. Bosilj, A. Milas, N. Pavin, I. M. Tolic, Microtubule sliding within briding fiber pushes kinetochore fibers apart to segregate chromosomes. Dev. Cell 43, 11-23 (2017). 